\title{
Automatic Generation of Assembly Sequence for the Planning of Outfitting Processes in Shipbuilding
}

\author{
Yan Wei
}





\section{Automatic generation of assembly sequence for the planning of outfitting processes in shipbuilding}

\section{Proefschrift}

ter verkrijging van de graad van doctor aan de Technische Universiteit Delft, op gezag van de Rector Magnificus prof. ir. K.C.A.M. Luyben, voorzitter van het College voor Promoties, in het openbaar te verdedigen op maandag 26 maart 2012 om 15.00 uur

door

$$
\begin{gathered}
\text { Yan WEI } \\
\text { 魏艳 }
\end{gathered}
$$

Master of Science in the Design and Manufacture of Ships and Marine Structures,

Wuhan University of Technology, P. R. China

geboren te Xiangfan, Hubei province, P. R. China 
Dit proefschrift is goedgekeurd door de promotor:

Prof. dr.ir. U. Nienhuis MBA

Samenstelling promotiecommissie:

Rector Magnificus,

Prof. dr.ir. U. Nienhuis MBA,

Prof. ir. J. J. Hopman,

Prof. ir. D. Stapersma,

Prof. ir. J. C. Rijsenbrij,

Prof. dr. ir. F. J. A. M. van Houten

Dr. ir. J. Coenen,

Prof. dr. ir. M. L. Kaminski voorzitter

Technische Universiteit Delft, promotor

Technische Universiteit Delft

Technische Universiteit Delft

Technische Universiteit Delft

Technische Universiteit Twente

Technische Universiteit Delft

Technische Universiteit Delft, reservelid

Keywords: shipbuilding, outfitting processes, assembly sequence, sequence generation, physical attributes, interference detection, mounting time

ISBN 978-90-6562-295-2

Published by VSSD, Delft, the Netherlands

Cover design: Nicolene van der Zwan

Copyright $(2012$ by Yan Wei

All rights are reserved. No part of the material protected by this copyright notice maybe reproduced or utilized in any form or by any means, electronic or mechanical, including photocopying, recording or by any information storage and retrieval system, without written permission from the author. 


\section{Contents}

1 Introduction 1

1.1 Current situation of European shipbuilding . . . . . . . . . . . 1

1.2 Removing the veils-Problem statement . . . . . . . . . 2

1.3 Approach the target-Research objectives . . . . . . . . . . . 6

1.4 Build the structure-outline of the thesis . . . . . . . . . . 9

2 Outfitting Processes in Shipbuilding $\quad 11$

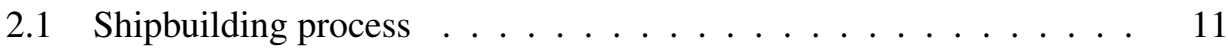

2.2 Definition of outfitting processes . . . . . . . . . . . 14

2.3 Characteristics of outfitting processes . . . . . . . . . 16

2.3.1 Interferences between yard and different subcontractors . . 16

2.3.2 Requirement of timely detailed engineering information . . 17

2.3.3 Existence of much ambiguity and tacit knowledge . . . . 20

2.4 Setting boundaries . . . . . . . . . . . . . . . 20

3 Outfitting Planning $\quad 25$

3.1 An overview of outfitting planning in shipbuilding literature $\ldots .25$

3.2 Current shipbuilding practice . . . . . . . . . . . 32

3.3 Integral outfitting planning . . . . . . . . . . . . 34

3.3.1 What is integral outfitting planning? . . . . . . . . . 34

3.3.2 Adoption issues . . . . . . . . . . . . . . . . 37

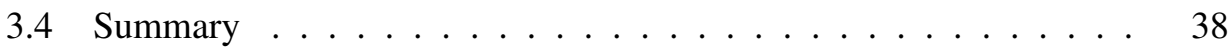

4 Assembly Sequence Planning Approaches 41

4.1 Introduction of assembly sequence planning . . . . . . . . . . 41

4.1 .1 General introduction . . . . . . . . . . . . . . . . 41

4.1.2 A proper assembly sequence . . . . . . . . . . . . . 43

4.1.3 Similarities and differences . . . . . . . . . . . . . 45

4.2 Automatic generation of assembly sequences-a literature review . . 46

4.2.1 The assembly sequence approach . . . . . . . . . . 47

4.2.2 The disassembly approach . . . . . . . . . . 51 


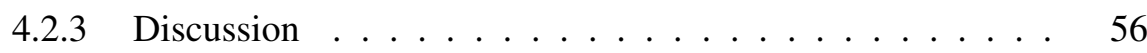

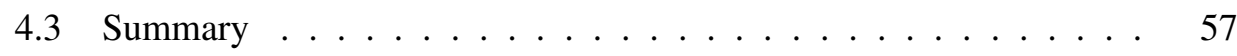

5 An Overview of the Structure of the Assembly Sequence Generation System

6 Physical Attributes Analysis $\quad 63$

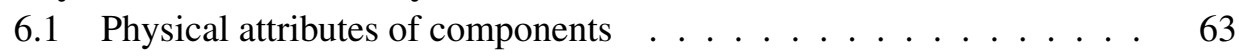

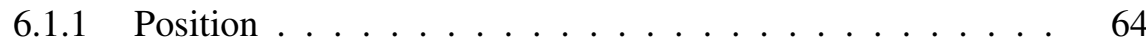

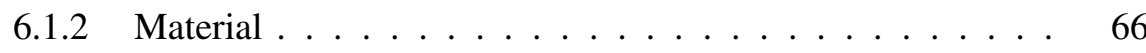

6.1 .3 Weight ...................... 68

6.1 .4 Size . . . . . . . . . . . . . . . . . . 69

6.1 .5 Penetration . . . . . . . . . . . . . . . . 69

6.1.6 Minimum work distance . . . . . . . . . . . . 70

6.2 The detection of interferences between outfitting components . . . 74

6.2.1 The application of the interference matrix . . . . . . . 75

6.2.2 Endless height iteration . . . . . . . . . . . . . . . 77

6.3 The relative importance of each physical attribute . . . . . . . . 79

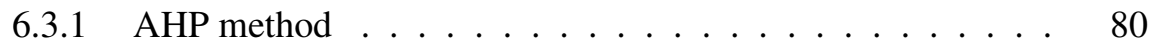

6.3 .2 Ranking . . . . . . . . . . . . . . . 81

6.4 Summary $\ldots \ldots \ldots \ldots \ldots \ldots$

7 Data Collection and Preparation $\quad 85$

7.1 Data sources . . . . . . . . . . . . . . . . . . . 85

$7.1 .1 \quad$ Raw data . . . . . . . . . . . . . . 85

7.1 .2 Data preparation model . . . . . . . . . . . . . 88

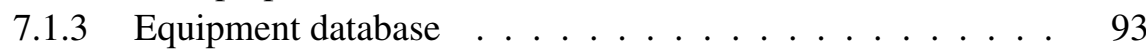

7.2 Summary . . . . . . . . . . . . . . . . . 96

8 Assembly Sequence Generation Model $\quad 99$

8.1 Description of the assembly sequence generation model . . . . . . 99

8.1.1 Generate assembly sequence in parallel by "Basket Done method"100

8.1 .2 The output of the model . . . . . . . . . . . . . . . 105

8.2 Added functionality . . . . . . . . . . . . . . . . 108

8.2.1 Resource leveling . . . . . . . . . . . . . . . . . . . 108

8.2.2 Component delay . . . . . . . . . . . . . . . . . . 111

8.2.3 Sequence generation by category . . . . . . . . . . . . . . 114

8.3 Summary . . . . . . . . . . . . . . . . . . . . 114

9 Model Application and Evaluation $\quad 117$

9.1 Mounting time verification . . . . . . . . . . . . . 117

9.1.1 The mounting time of pipe spools . . . . . . . . . . . 118

9.1.2 The mounting time of HVAC ducts . . . . . . . . . 120 
9.1.3 The mounting time of cable trays _ . . . . . . . . . . 121

9.2 Assembly sequences selection . . . . . . . . . . . . . 123

9.3 Analysis of test cases . . . . . . . . . . . . . . . . 126

9.3.1 Four test cases . . . . . . . . . . . . . . . . 127

9.3.2 Discussion of the assembly sequence and duration . . . . 130

9.4 Limitation of the model and possible improvements . . . . . . . . 138

9.4.1 Obtain the steel structure information . . . . . . . . . 138

9.4 .2 Installation of equipment . . . . . . . . . . . . . . 139

9.5 Conclusion . . . . . . . . . . . . . . . . . . . 141

10 Sensitivity Analysis 145

10.1 The sensitivity of the weighting coefficients . . . . . . . . 146

10.1.1 Explore the acceptable weighting coefficients . . . . . . 146

10.1.2 Measure the sensitivity . . . . . . . . . . . . . . . 147

10.1.3 Analyze the sensitivity . . . . . . . . . . . . . . . 148

10.2 The sensitivity to the minimum work distance . . . . . . . . . 154

10.3 The analysis of two versions of assembly sequences . . . . . . . 157

10.4 Summary . . . . . . . . . . . . . . . . . 158

11 Conclusions and Recommendations 159

11.1 Conclusions . . . . . . . . . . . . . . . . . . . . . . 159

11.2 Recommendations . . . . . . . . . . . . . . . . . . . . 161

\section{Appendices}

A The derivation of relative importance of the five physical attributes 163

B Preliminary estimation of mounting time 167

$\begin{array}{ll}\text { C UML class diagram } & 169\end{array}$

D Flowchart of the Basket Done method 171

E The background of the questionnaire people 175

F The animation of the assembly sequence of test case S1 177

$\begin{array}{ll}\text { Bibliography } & 183\end{array}$

List of Abbreviations 189

$\begin{array}{ll}\text { List of Figures } & 191\end{array}$

$\begin{array}{ll}\text { List of Tables } & 195\end{array}$ 
Summary

Samenvatting

201

Acknowledgments

205

Curriculum Vitae 


\section{Chapter 1}

\section{Introduction}

\subsection{Current situation of European shipbuilding}

The strategic nature of the shipbuilding industry encouraged many countries to develop domestic capabilities to build ships without necessarily taking into consideration the developments in the world market. The most prominent example is South Korea, and more recently (in 2009), China which accounted for $28 \%$ of the world production (compared to Korea $32 \%$ and Japan $21 \%)^{1}$. In the last three decades, Asian players have risen to take over most of high-volume, relatively non-complex ships like oil tankers, bulk ships and container ships.

European yards have been more careful in their business development and have largely refrained from massive capacity expansions ${ }^{1}$. It is becoming more visible that the focus on niche markets placed the European yards as leaders in the construction of complex and high-valued vessels such as large cruise ships, ferries, mega-yachts, support vessels and dredgers, which are highly customer designed and in which advanced and often new technology is used. Since the technology is easily transferable and, at the fabrication level, it does not require a high level of education or highly productive skills given the wage differences, European shipyards continue to lose market share in shipbuilding production and cannot compensate their high labor costs and cannot combat aggressive competition from efficient Asian yards using the same techniques.

To secure and enhance the competitive position of European shipyards in a sustainable way, attention needs to be given to the ongoing innovation of products and production processes. This is especially true for high-value added ships of which a larger part of the building cost is related to engineering, equipment purchase and installation. Only a limited number of product innovations (often relating to hydrodynamics, hull structures, power plants, functional equipment and cargo handling and

\footnotetext{
${ }^{1}$ Community of European Shipyards' Association (CESA), annual report 2009-2010
} 
2 Automatic generation of assembly sequence for the planning of outfitting processes in shipbuilding

mooring) are implemented in each shipbuilding project. This is because the financial consequences of these innovations need to be calculated at the initial tendering stage and the results need to be sufficiently accurate in order to allow for their adoption (Hopman, 2007). However, in many cases there is not enough time for this. Ships are usually ordered on the basis of conceptual designs which have been created within a few weeks. Thus, introducing too many product innovations in one particular shipbuilding project creates too many risks. Improvement of the production processes yields more sustainable results for shipyards. This is caused by the fact that many production process innovations are not easily copied. It is therefore not surprising that in shipbuilding, innovation of the use of improved technology in the production process is a must for European shipyards to compete against the low-cost countries.

The production process is essentially one of assembly, and few of the individual tasks require sophisticated technical skills, though some automation of cutting, welding and repetitive assembly is possible. The main challenge lies in planning, organizing and executing the tens of thousands of operations that contribute to the production of a ship. In order to promote innovative manufacturing management and improve production process control, it is necessary to explore more rationalized and reliable production planning methods, especially in outfitting processes. In a modern passenger cruise vessel for example, the outfitting work can represent, in terms of cost, up to $80 \%$ of the total production work, the steel work being only $20 \%$ (Andritsos and Perez-prat, 2000). In Germany (2005), outfitting processes represent about $38 \%$ of the construction work in the building industry while the main construction activity represents only about $30 \%$ (Konig et al., 2007). Any improvement in outfitting processes would yield substantial benefits for the shipbuilding industry.

\subsection{Removing the veils-Problem statement}

Outfitting work in European shipbuilding industry involves a close network of highly specialized companies, suppliers and shipyards. In countries outside Europe there is hardly any network of this kind (LeaderSHIP2015, 2003). This is due to the use of outsourcing and subcontracting ${ }^{2}$, a widespread strategy of western shipyards (Muller, 2007). Nowadays it is assessed that 50-70\% of the value added comes from external subcontractors and suppliers, whereas for more complex ships this can be as high as 70-80\% (IKEI, 2009).

This strategy allows the shipyards to focus on their core competencies which, in most cases, include the organization of the assembly of the vessel. As a consequence, functions which are deemed less profitable or highly specific are passed on to external companies. Tasks that were usually integrated into the own production process but

\footnotetext{
${ }^{2}$ Outsourcing is to transfer people or assets to a third-party provider. It is more of a business process than a procurement process. Subcontracting is to transfer a process alone. (See 'Subcontracting versus outsourcing' on the website 'http://www.supplymanagement.com')
} 
which are nowadays frequently performed by subcontractors include most parts of the outfitting work, like piping, HVAC and electrical installation.

The advantages of this strategy for shipbuilding clearly are the increased capability to react flexibly to rapid changes in demand and the reduced fixed labor costs. However, the long-term implications of using outsourcing and subcontracting are quite ambiguous (Muller, 2007) . One of the several critical areas is that their use increases the need for a proper coordination of the work performed by the various subcontractors. Very often the work performed by one contractor depends on the work of another contractor. Thus, if one contractor fails to comply with deadlines and certain quality standards, all other contractors run the risk of increased costs and a delay in the delivery of the final product.

Currently, planning of outfitting processes is not sufficiently considered. In practice, processes seem to proceed somewhat uncoordinated. Yards fulfill the role of project coordinator and generally provide the subcontractors with a general time frame for outfitting. Meanwhile, each subcontracted company plans its own work in more detail, independent from other executers. Consequently, outfitting processes exhibit interferences and disturbances, which may frequently cause conflicts, rework and long delays on the work site. This affects adversely the efficiency of the work. Figure 1.1 and figure 1.2 show two negative examples on the work site. Generally, outfitting processes are characterized by a great interdependency and have different requirements with respect to accessibility and associated surrounding area.

These circumstances create an urgent need: in the planning of outfitting processes, how to develop an integral and reliable planning which strengthens the cooperation among the involved parties and reduces the conflicts on the site?

Another potential problem caused by the heavy use of outsourcing and subcontracting is the loss of industry-specific knowledge and skills. This is an issue that is particularly important for shipbuilding, which is a high tech industry depending on well-educated and highly skilled workers. For example, the assembly work of outfitting is guided by complicated drawings in the field. Workers form a mental picture of the completed arrangement from the drawing; consider the assembly procedure of parts; visually confirm the availability of the necessary parts; consider the transportation method, such as a crane; envisage the necessary tools and then carry out the assembly work. Finally, the accuracy of the assembly is confirmed by comparing it with the drawing. These jobs require much experience and skills.

Meanwhile, ageing of the workforce is a problem that should also not be underestimated (see figure 1.3a and figure 1.3b). Generally speaking, the North-West European countries have a relatively older workforce compared to the South-East European countries. Finland, the UK, the Netherlands and France are expected to experience the largest problems with the aging of the workforce, since they have relatively many employees over the age of 55 years (Group, 2009). One of the typical solutions is to provide specific in-house training. Nevertheless, the transfer of knowledge from 
4 Automatic generation of assembly sequence for the planning of outfitting processes in shipbuilding

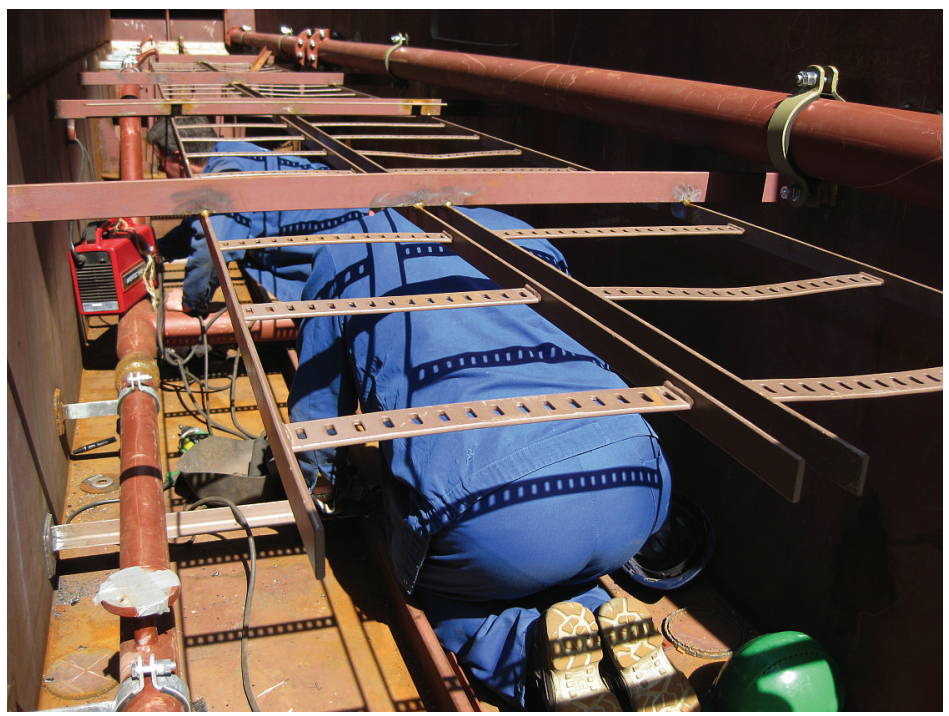

Figure 1.1: Awkward work position to install HVAC ducts

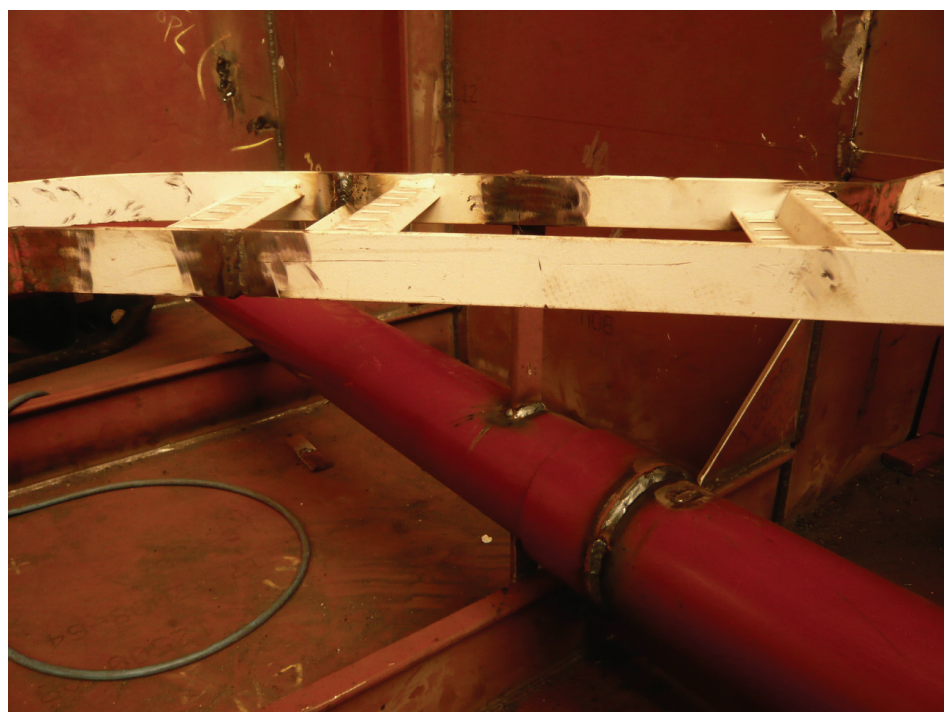

Figure 1.2: Collision between the support of cable trays and steel pipe 


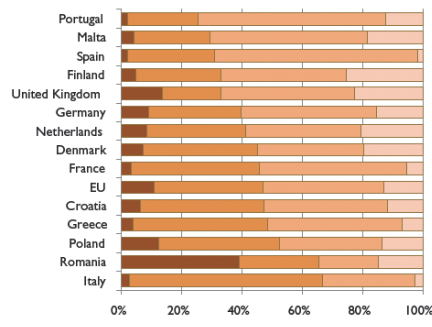

(a) Current situation

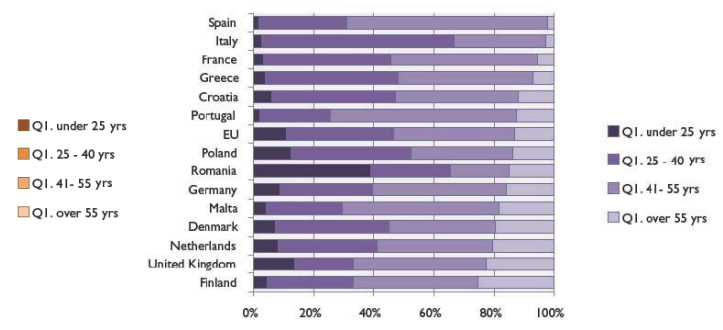

(b) Outlook over 10-15 years

Figure 1.3: EU-14 Shipbuilding technical workforce-age distribution (outlook over 10-15 years) (reproduced from Hart (Hart and Schotte, 2008))

the most skilled employees to those less skilled is often not sufficiently systematized. Experienced workers with a long professional work history often do not consider issues that are evident to them as matters that should be collected and saved. Also not everyone is able to think of his own work unique and to report and verbalize own occupational skills, orally or in writing (Granger, 2008).

Both aspects point to another question in outfitting processes: how to make expertise explicit through codified information so that years of experience may remain and be used by novice workers?

In addition, since outfitting processes involve installing various components in specific locations on the site according to technical specifications, the right materials should be delivered to the right place before installation can take place. This is especially true for piping processes which are identified as being critical to the success of numerous industrial projects. The successful application of palletizing helps to sort pipe pieces as soon as they are fabricated. However, within the pipe pallet, all pipe spools ${ }^{3}$ belonging to the same section are piled in a disorderly fashion. The ones at the bottom of a pallet may need to be assembled earlier. Thus it takes workers quite some time to dig them out. The example in figure 1.4 forced two workers to spend 35 minutes searching and transporting one pipe spool in the pipe pallet to the work site.

Thus, efficient outfitting processes rely on proper logistics. This raises the question: how to sequence material deliveries based on their assembly order? But indeed, this requires having an assembly order in the first place.

\footnotetext{
${ }^{3} \mathrm{~A}$ spool is another name for a piece of pipe, a piece of HVAC duct and a piece of cable tray but cannot be further split into smaller units. Workers install a spool as a single component on the worksite.
} 


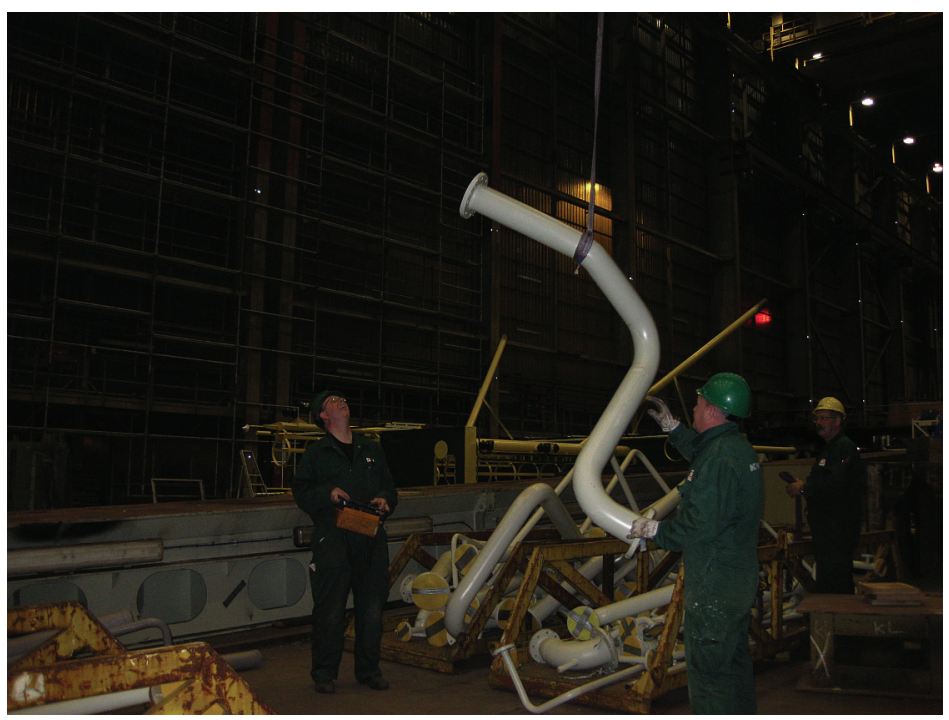

Figure 1.4: Search pipe spools in a pipe pallet

\subsection{Approach the target-Research objectives}

When hidden problems in outfitting processes gradually emerge, pushed by intense competition in the shipbuilding industry, an innovative attitude is essential. Given the background of the subcontracting and considering the complex nature of the outfitting processes in shipbuilding, this research brings forth a novel approach to the automatic generation of assembly sequences for outfitting items across different disciplines.

The CAD model is the basic input for the research. It is more than the collection of geometric models representing the shapes of all kinds of components in a ship. It also contains much implicit assembly knowledge. For example, in the low temperature (LT) cooling system in a 3D model, a LT-charged air cooler is connected to a lubricating oil cooler via steel pipes and valves. When assembling this cooling system, it is not preferable to install the air cooler and the oil cooler first and then insert pipes and valves in between, because it incurs the difficulty of connection. To translate this knowledge into practical assembly work requires heavy involvement of human mental powers. A product drawing tells an experienced worker much about the production process. In principle it should be possible to harvest this implicit knowledge and turn it into an explicit method.

Thus, the hypothesis of the research is that it is possible to automatically generate assembly sequences for ship outfitting based on the product model obtained during the detailed engineering phase.

The purpose of the research is to develop an automatic sequence generation method which is able to give the yard and subcontractors a realistic and reliable outfitting plan that identifies the relationships among outfitting activities, estimates a 
realistic mounting time, and displays it by means of animation.

The research was split up in the following specific objectives:

- To capture the assembly knowledge in terms of technical aspects

A fundamental aspect of creating an assembly sequence is deciding which component to do next. This requires sufficient attention to be paid to: what activities are carried out in what order with what resources during the outfitting stages, collectively named the 'assembly knowledge'.

The execution of the assembly of a particular component is largely influenced by its physical attributes and the availability of required resources. The 'assembly knowledge' can be categorized into technical aspects and resource aspects. Technical aspects explain what kind of physical attributes of the product affects its mounting order and how. Resource aspects refer to how the amount and qualification of resources, such as machines and employees, influence the product's mounting order.

The type and capacity of resources vary depending on the size of a shipyard. To schedule them in a way that adapts to a specific assembly case becomes possible by the availability of suitable planning tools. While resource limitations influence the duration of activities, it should not hamper its efficiency. Thus, ideally, resource should be adapted to the nature of the work to be done. Therefore, this research mainly captures the assembly knowledge in terms of the technical aspects on the work site. It is one of the indispensable prerequisites for realistic assembly sequences.

- To fill the gap between engineering and planning

Here 'engineering' specifically means the detailed engineering phase, following the systems engineering phase. It generally includes making production drawings for the construction and pre-production departments, for pre-outfitting of sections and the outfitting of the erected ship. Planning states when things will be done, adjusting resources according to the actual production needs. It aims for the fulfillment of the construction milestones established during the sale phase.

Although currently the availability of the tools largely accelerates both the engineering and planning processes, to convert the engineering documents (e.g. 3D models, production drawings, a list of components) into a reliable planning still heavily relies on a planner's experience. This research aims to bridge the gap between engineering and planning work.

- To provide insight into the interdependencies of assembly work for a more detailed production planning in order to optimize the production process control 
8 Automatic generation of assembly sequence for the planning of outfitting processes in shipbuilding

The nature of outfitting processes can be portrayed as interferences, disturbances and great interdependencies. It makes that the minimization of resources and the reliable adherence to a tight schedule is challenging. The dependencies between section/block construction with its internal diversity of components, the assembly processes and the involved resources are just too complex and variable for a human planner to control without proper tooling. A more detailed production planning, with reasonable estimates of throughput time and usage of resources would, to a great extent support the production processes and reduce the risk of overrunning the budget and time.

Figuring out the dependencies of assembly work in outfitting processes would provide a planner with an indication of how resources should be deployed and how long the assembly of a series of specific components would take. The research deals with how and why outfitting activities are related.

- To present a starting point for the simulation of outfitting processes.

Simulation is a very helpful and valuable work tool in manufacturing. It can be used in the industrial field allowing the system's behavior to be learnt and tested. For example, a distributed simulation system is developed to support the design and control for container handling in the port (Boer et al., 2002); fatigue simulation methods are presented to assess the fatigue life of the seam welds joining the vehicle components and body structure (Hung, 2002). It is also known that the application of simulation technology in production processes of shipbuilding industry helps to increase the planning reliability and robustness as well as minimize risks. Because the major impacts of the product, the building method or other constraints on the production process can be analyzed dynamically, possible bottlenecks are detected and avoided in advance (Steinhauer, 2005, 2006). However, detailed simulation of outfitting processes still remains challenging for two reasons: first of all, the complex behaviors of the outfitting processes have to be modeled and secondly the data about the outfitting parts are often not at all or not sufficiently available (Steinhauer, 2011).

In the research, we retrieve the outfitting parts' data and reorganize them in such a way that they can be accessed for simulation purpose. Also, in many cases, a planning is used as input for simulation analysis and our research yields just that.

In order to accomplish the above objectives, next to the literature study, most of the effort has been spent on developing a suitable method and subsequently test it in practical situations obtained in the field. Observations were necessary for understanding real production processes. These were done on the work site in one of the leading shipyards in the Netherlands which has a close collaboration with a pool of specialist co-makers. In order to record the actual work processes, the observations were maintained for a prolonged period. Besides, interviews with workers, foremen, 
outfitting coordinators, project planners and project supervisors have been carried out through the whole research period.

\subsection{Build the structure-outline of the thesis}

To describe the research done to realize the objective, the thesis is divided into three parts, each including several chapters.

Chapters 2 to 4 are introductory chapters providing backgrounds. They begin with the overview of outfitting processes in shipbuilding. Since it is not possible to investigate everything relevant to outfitting processes in this research, the research boundaries have also been explicitly described in chapter 2. After the introduction of current practices of outfitting planning and establishing both the strengths and weaknesses, chapter 3 puts forth our concept of integral outfitting planning. Chapter 4 contains the summary of the literature review. It mainly deals with the methods that are used to generate assembly sequences in the mechanical industry, and the possibility of their application in shipbuilding outfitting processes.

Chapters 5 to 9 are the main body of the research dealing with the research into an automatic generation system of assembly sequences. Chapter 5 presents a holistic view of the automatic generation method of assembly sequences. Chapter 6 is devoted to the analysis of physical attributes of outfitting components that influence their mounting order, and the mathematical way to represent them. Once these attributes are clear, data collection and preparation are addressed in chapter 7 . It describes the availability of these data and how to make them useful as input, ready to be accessed by the model to be established later. Chapter 8 mainly focuses on how a sequence generation model works and what results can be obtained. Chapter 9 presents the procedure of validating the assumptions made during the model development and the results generated with the help of our method. On the basis of four realistic test cases, the validity of the model is discussed. Some application examples are also included, which shed further light on the nature of outfitting processes and the potential use and current limitations of the method.

Chapter 10 focuses on the sensitivity analysis. It is geared to analyze the sensitivity of the assembly sequences to the input parameters. Finally chapter 11 presents the conclusions and recommendations of this research. 



\section{Chapter 2}

\section{Outfitting Processes in Shipbuilding}

To build ships the shipyard, together with the project partners must accomplish three main tasks - the design and engineering of the ship, the construction of the steel structure, and the outfitting by installing various systems and equipment that allows the ship to operate and perform various missions. After introducing the position of the outfitting process in the whole shipbuilding process in section 2.1, section 2.2 gives the definition of 'outfitting' and illustrates its integral relationship with steel construction. Its present-day characteristics are introduced in section 2.3. Since it is not possible to view everything about outfitting processes in this research, section 2.4 explicitly describes the research boundaries.

\subsection{Shipbuilding process}

Shipbuilding, which dates back B.C., has been growing as an important and strategic industry since the 20th century. Nowadays, it is an extremely complex business, which involves shipyard, owner, co-makers, suppliers, class, authorities and specialist consultants and their interactions. The time between order and delivery is very short, which means that a very large number of tasks must be performed in parallel. The ship in many cases is a one-off product and is handled as a distinct project. The processes are recognizable, but vary slightly from shipyard to shipyard. The process of shipbuilding, conducted in one of the modern shipyards in Europe, is shown in figure 2.1. Each block represents a phase in the process.

The shipbuilding processes start with the pre-contract design. It provides sufficient information for a basic techno-economic assessment of the alternatives to be made, fills out the arrangements, describes the expected service performance, details the systems agreed with the owner and satisfies the building contract conditions including the major planning milestones. At this stage the builder should have sufficient 
12 Automatic generation of assembly sequence for the planning of outfitting processes in shipbuilding

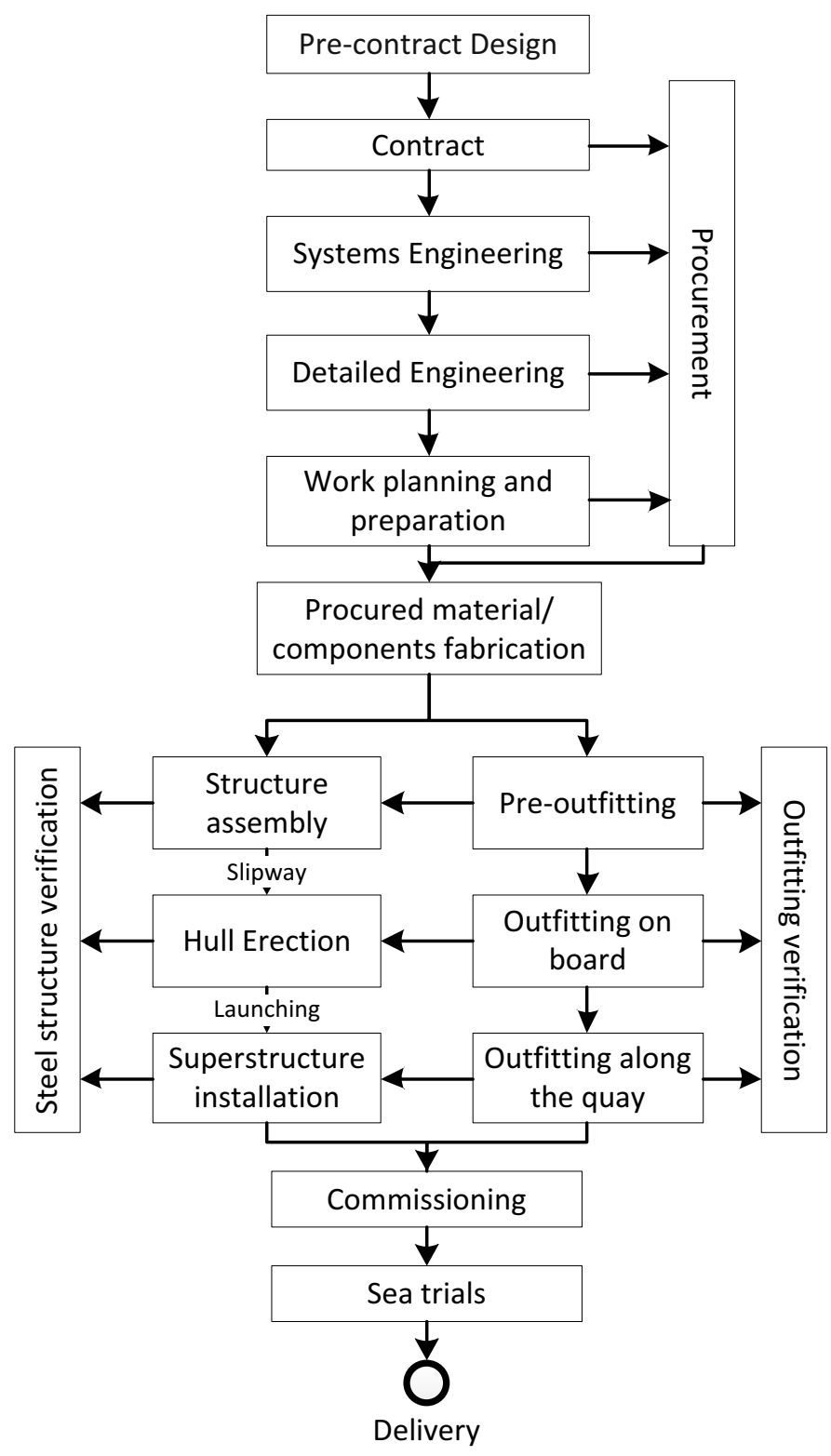

Figure 2.1: Main stages in shipbuilding process 
information to tender.

After the contract in which the construction milestones ${ }^{1}$ are set up, systems engineering starts. It is a process of transformation of a general specification and set of requirements into a complete description of a physical product that matches this specification. It includes the detailed specification of the main structural elements, the specification and arrangement of all main systems and specification of parts and properties for purchase and the layout of compartments (Coenen, 2008). In the detailed engineering phase, the required output and deliverables are well-defined, for instance by drawing lists and equipment lists.

Work planning and preparation is the stage to establish the building plans, including small iron work building plans, section building plans, hull erection plans and pre/outfitting plans. Its goal is to guarantee the fulfillment of the construction milestones. In some shipyards and market conditions, procurement starts even before signing the contract, considering the long-lead-time items and some advance construction work. It is an ongoing process throughout the whole production process if the smallest items, like bolts and nuts, are taken into consideration. But in general, the major items are purchased during the engineering stage and work preparation stages.

The fabrication of procured material/components includes marking, cutting and bending steel parts, transforming steel tubes into pipes and steel bars into cable trays, assembling metal panels, electrical circuits and special (standard) components to electrical switchboards, etc. It is the step to make sure that all components of a ship are in conforming with the specifications and have the shapes required by engineering drawings.

Production processes follow two lines: the line of the steel structure construction and the line of the non-structure component assembly. Along the first line, steel structure assembly often including panel assembly, section assembly and block assembly, hull erection and superstructure installation take place chronologically but partly in parallel. For example, the panel assembly of one section may be proceeding simultaneously with the erection of another section on the slipway. The non-structure component assembly commonly called 'outfitting', needs to be differentiated from the steel-structure construction. In which building stage 'outfitting' happens and how it relates to the structure construction will be explained in section 2.2.

Along the production processes, the verification work is proceeding. The steel structure verification is used to check whether the dimensions of steel parts are correct, whether the type of welding is according to the quality control standards, whether tanks are watertight, etc. The outfitting verification is used to check whether the out-

\footnotetext{
${ }^{1}$ In the contract, often there is an agreement on a number of progress payments to be made when certain milestones have been passed. These milestones could include: the cutting of the first steel plate, the laying of the keel, completion of the hull, installation of engines, tanks and generators, launching, installation of the superstructure, completion of interior finishing, completion of sea trials, and expiry of a trouble free period since delivery (Caldwell, 2002).
} 
14 Automatic generation of assembly sequence for the planning of outfitting processes in shipbuilding

fitting components are the same as required by the specifications and drawings when they are delivered, to test basic functions of equipment after they are installed (if possible), to test whether there is leakage on pipes, etc. The verification work is done by the quality control department of a shipyard and its co-makers and will be independently checked by the personnel from classification society and also by the representatives of ship owners.

During commissioning, the ship comes alive and is made ready for sea trials and delivery. Of course, in order to save time, an overlap often exists between phases. For example, when the detailed engineering of the first section of a ship is done, the production preparation of this section can start and thus, the steel parts of this section are immediately fabricated. In such a case, there is no need to wait until the engineering of the whole ship is finished before starting the production of the first section.

\subsection{Definition of outfitting processes}

In the ship construction processes, outfitting usually refers to two distinctive but closely linked phases, i.e. Pre-outfitting and Outfitting on board. Pre-outfitting, generally refers to installing piping, electrical components, HVAC components and other system components (engines, winches, blowers, switchboards, etc.) in the panels, sections and blocks making up the hull structure. This work, generally, is performed inside the specialized and well-equipped workshops with ideal lighting and access (Maffioli et al., 2001). Outfitting on board continues these activities, which have not yet been finished in the previous pre-outfitting stage, or embarks on new activities that can only be done during the hull erection on the slipway or after launching alongside the quay. Examples are equipment alignment and further connection work. In this thesis, the term 'outfitting processes' is the general description of both. Special occasions will be distinguished by either 'Pre-outfitting' or 'Outfitting on board/ along the quay'.

Clearly, outfitting can never be separated from the hull construction. Figure 2.1 shows the demarcation of 'pre-outfitting' and 'outfitting on board/ along the quay' along the steel structure building process.

Outfitting that occurs during the assembled ship stage requires workers to move to the dry dock, slipway, or land-level facility, bringing with them the materials and equipment and their construction tools. Working conditions are not ideal because of factors such as difficult access, limited space, and difficult work positions (e.g. overhead welding). The workplace is typically congested, with high material flow costs, and often more hazardous conditions. This adds to the indirect time required to perform an assembly task. It is now widely accepted that many of these problems can be relieved to some degree by installing more components earlier during the preoutfitting stage where the materials and equipment are close at hand and steel sections 
can be positioned in the best way to allow easier access and installation. However, so far there is no clear boundary to identify which specific activities should be done during pre-outfitting or which during outfitting on board. Briefly, the choice of the best stage to perform the assembly work ${ }^{1}$ depends upon (amongst others)

- Production schedule- In order to meet milestones in the shipbuilding process, the section building plan and hull erection plan have been established, which have a profound influence on the decision of how many weeks are given to execute the assembly work during the pre-outfitting stage. In general, if time is allowed, experienced foremen at shipyards reported that, it is possible to assemble $80 \%$ of the pipe spools during section building.

- Space accessibility- Some assembly work must be done during the building of the steel structure construction stage, since it would be very difficult and expensive to do those tasks later during the assembly stage. An example is installing pipe work in double-bottom sections of dredgers, which could not feasibly be done after the sections are built.

- Lifting capacity-The facility layout is the root for the choice of the optimum section and block handling capacity. A large lifting capacity is a significant advantage because the larger the prefabricated hull sections, the more assembly work can be transferred from on board to the workshop.

- Material availability- In-time material delivery is one of the key factors contributing to a high-percentage pre-outfit process. Because of the unavailability of material during the section building stage, a one-day assembly job may require one week in a later stage.

- Section-building position- The section-building position, upright or upside down, decides what outfitting component can be assembled in that particular situation. For example, when the section is upside down, big and heavy pieces of equipment are hardly installed on the deck because they need extra lifting, securing and protection to prevent damage when the section is turned to the upright position. They are often installed when sections are put on the slipway in the upright position. However, when the section is upside down, outfitting components which are closer to the ceiling of the section are preferably installed because of easy handling and the possibility of under hand working.

Of course, the organization of outfitting processes has evolved against the background of the current shipbuilding strategy. It tries to optimize the steel structure work and occupy the dock/slipway as briefly as possible. As the amount of outfitting

\footnotetext{
${ }^{1}$ Assembly work in this book means the installation of outfitting components and does not refer to the steel structure construction.
} 
16 Automatic generation of assembly sequence for the planning of outfitting processes in shipbuilding

work grows resulting from complex and high-value vessels, the necessity of a new shipbuilding approach which emphasizes earlier and easier outfitting work gradually emerges.

It might not be impossible to build a ship starting from interior outfitting work to the outside hull structure construction ('inside-out'). It means that as soon as the bottom deck of a ship has been laid on the slipway, the outfitting work including installing equipment and carpentry work on that deck can start. When the outfitting work is finished, the starboard and portside can be closed by placing and welding hull steel plates. Then, another deck is put on, followed by the accomplishment of all outfitting work belonging to this deck and closing it by constructing the hull structure. The rest of the ship is built in the same manner. At the end, the last piece to put into the ship is not a piece of equipment but a steel plate and there is no need to perform further outfitting along the quay any more.

In this way, there is no so called 'pre-outfitting' because traditional section building and erection do not exist. All outfitting work is done in the dock/slipway. The practicality of the new approach is not immediately clear, but as a thought experiment it sheds light upon the future shipbuilding strategy which undoubtedly should focus on better ways to carry out outfitting work.

\subsection{Characteristics of outfitting processes}

Today, the relative importance (at least in terms of cost) of the steel cutting, shaping and assembling has decreased substantially. This is true especially for the high value-added small series or one-of-kind ships where the western European shipbuilding industry still holds a considerable share of the market. The focus of shipyards has thus shifted from the pure steel construction towards extensive outfitting work. Any improvement in the outfitting process would yield substantial benefits for the shipbuilding industry. The logic is simple: completing outfitting earlier allows the work to be done in better conditions. If outfitting work is done in better conditions, in a less cluttered environment such as in a work shop, it can be done with fewer men in less time with better quality and corrosion prevention; thus the work is done more efficiently. All this leads to the reduction of time and cost.

It is easy to see that improved outfitting processes should pay off. Nevertheless, many shipyards have difficulty achieving it. There is clearly a need to investigate why some shipyards find this very challenging.

\subsubsection{Interferences between yard and different subcontractors}

The majority of EU shipyards that were surveyed (Schank et al., 2004) use total subcontracting extensively, maintaining in-house capabilities primarily in the steel structure area. For example at Meyer-werft in Germany, the outsourcing rate of the 
ships under construction is some $75 \%$. This implies that many parties are involved in the outfitting processes. There exists not only the vertical relationship between a yard and a subcontractor but also the horizontal relationships between the subcontractors themselves. Figure 2.2 provides the use case diagram to describe the relationships among outfitting entities for a particular example of boiler installation. In figure 2.2, this falls under the category of 'HVAC'.

After the finalization of its specification which has been detailed by the engineering department and approved by the ship owner, the purchase department from the yard asks the HVAC subcontractor to buy and deliver it in a certain place and on a certain date. The HVAC subcontractor contacts the boiler supplier and negotiates the specific delivery time and price. Upon delivery and acceptance, the finance department processes payment. If the section in which the boiler is located is outsourced to another company and if the boiler is to be pre-outfitted, the HVAC subcontractor must inform the boiler supplier to deliver it to the appointed work site. Since the boiler is part of a water central heating system which consists of the boiler, the radiators and the interconnecting piping, the piping company must make sure that there are no pipes in the way when using the crane to lower down the boiler on its foundation from above. This foundation is installed by the production department in the yard and painted by the conservation company. Besides, within the boiler, there are sensors used to measure the temperature. They need wires to be connected to the electrical power by the electrical company. No matter in which stage the boiler is installed, it must fit in the building plans established by the planning department.

Currently, in order to update the outfitting progress and control root causes of variability, weekly meetings are conducted in which all subcontractors and the outfitting coordinator from the yard participate. However, this does not prevent the necessity of extensive coordination efforts on the production site to handle day-to-day work. According to the authors' observation, the communication is often informal and not extensively documented, making the management of a detailed planning of outfitting processes more difficult.

\subsubsection{Requirement of timely detailed engineering information}

Production performance depends largely on the quality, quantity, and timeliness of technical information supplied by engineering. Large investments in the preparation of timely and accurate engineering information are required in order to reduce the production time during the outfitting stages. For example in figure 2.3, four pipe holes in a plate have not been burnt when cutting the plate because of the unavailability of the information. It took two pipe fitters three hours at a later stage during production to burn them, including waiting for the crane to transport a welding machine, preparing the proper welding torch and finding the positions of these holes in the steel plate.

We also want to address the important roles that classification societies and ship 
18 Automatic generation of assembly sequence for the planning of outfitting processes in shipbuilding

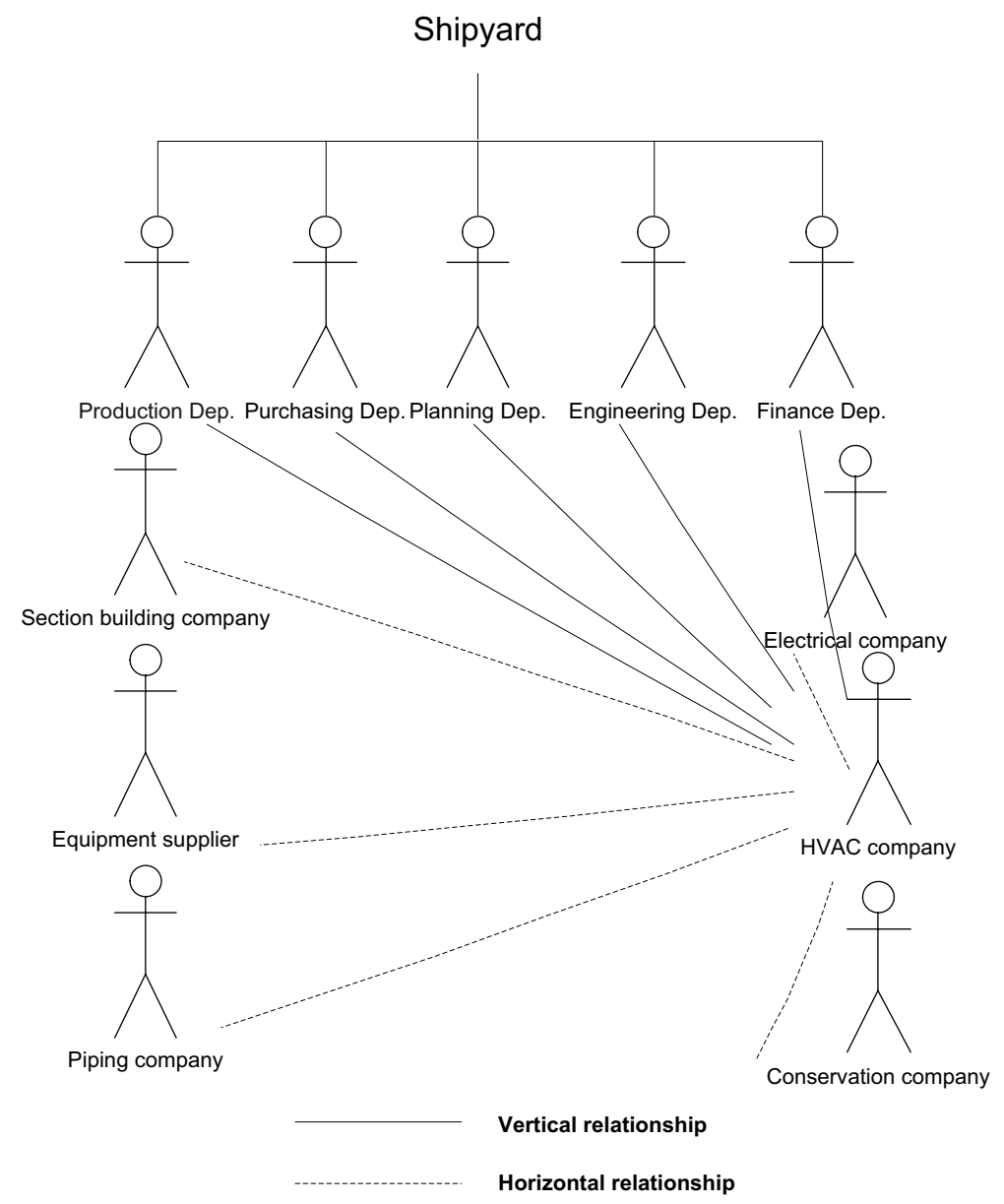

Figure 2.2: Use case diagram for outfitting entities 


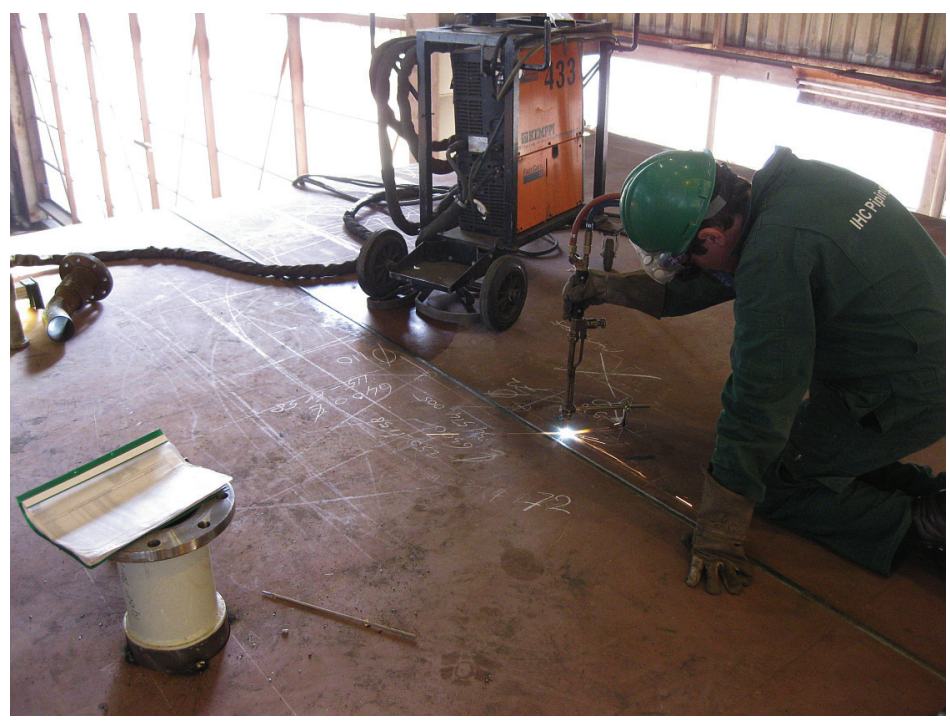

Figure 2.3: The worker burns the pipe holes

owners play in the delivery of the detailed engineering drawings. Although ship owners enjoy complete participation in the pre-contract design, they continue to be entitled to be actively involved in the basic engineering stage before all the necessary drawings are sent to classification societies. This is especially true for tailor-made one-of-kind ships. Only after the classification society has approved all the required drawings, the engineering department can start formally to do the detailed engineering. However, due to time constraints, the yard will often press on without formal approval, possibly incurring some rework at a later stage. Thus, the good cooperation with ship owners and the timely approval from societies together benefit the shipyard to deliver the precise, detailed and required engineering information in time.

For many years the necessary level of detail of engineering information is a topic of much controversy, due to the precarious balance between the large initial investments and the uncertainty of the final benefit obtained by subcontractors. In most situations, the proper balance largely depends on how much effort all involved parties are willing to invest. A rather narrow viewed concentration of each co-maker on its own part is bound to limit the level of detail of production management and planning, both of the yard and the co-makers. This may undermine the controllability of outfitting processes and cause overrun of the budget and throughput time.

Here is an example stated by someone in the field: in previous years, considering that too many man-hours would be spent on engineering work, pipes supporting lighting arrangements were not added in the 3D model. This caused at least 50\% of these pipes installed during the pre-outfitting stage in the engine room to be erroneously removed by another subcontractor during the section assembly stage because 
20 Automatic generation of assembly sequence for the planning of outfitting processes in shipbuilding

they blocked the route of other pipes that were routed and added in very late. The loss the co-makers suffered became twice as much as the investment that they would have had to put in at the beginning of the engineering stage.

\subsubsection{Existence of much ambiguity and tacit knowledge}

Much ambiguity and tacit knowledge exists in shipbuilding, especially in outfitting processes. For example, after a certain percentage of building a steel section has been completed, workers from different outfitting companies enter into the section and start to do all kinds of mounting work. But what is the right percentage? If outfitting work starts too early, steel construction work, like welding, may interfere with pipe mounting work. If it starts too late, getting components in sections may become complicated because of the obstructions from structural components. Also time may be too short to achieve a high pre-outfitting percentage. Normally, planners assume $70-80 \%$ of a section to be finished before allowing start of pre-outfitting work. But also another major constraint, the nature of a section (e.g. double bottom or superstructure) also complicates the decision-making process. To extract these kinds of invisible 'rules' requires much experience or thorough investigations. It is necessary to make knowledge explicit through codifying experience in order to promote innovative manufacturing management and engineering. According to the observations by the author and interviews with subcontractors, planners quite often use rules of thumb abundantly to make important decisions.

Furthermore, the current assembly work in outfitting itself very largely relies on skillful workers, who quite often possess more than ten years of experience. Their expertise helps them visualize a three-dimensional image and corresponding assembly procedure by observing a two-dimensional drawing. However, this is very difficult for workers with less experience because there are various components and the shape is complex. They often make mistakes and have to perform rework. To convert tacit knowledge to explicit knowledge by codifying the outfitting assembly process may help to enable efficient assembly regardless of work experience and thus, to a certain extent, soothe the aging problems of the work force.

\subsection{Setting boundaries}

From the definition of the outfitting process, it is not hard to see that in terms of timespan, it spreads almost across the whole ship production process, from the section assembly all the way to the delivery of a vessel. In terms of work content, it covers a broad range of functional tasks, including structural (installing equipment foundations, doors, ladders, etc.), piping, electrical power distribution, HVAC, joinery, painting and insulation. Within the scope of this research, it is neither necessary nor possible to dive into every aspect of this process. Consequently, it is indispensable to set boundaries to make sure the essential problems stated in chapter 1.2 are 
sufficiently dealt with.

1. The boundary of building stages: the outfitting processes as covered in this research start with the section building stage and ends with the hull erection stage.

Most of onboard outfitting assembly work occurs within this period to make sure that the whole underwater part is ready before the vessel is launched. Activities on the critical path, like the installation of all components which are under the waterline and a considerable part of cable pulling and connection work, should be carried out during the outfitting processes thus defined. Thus, it is worth investigating within this period how to improve the assembly work efficiency and by what way. Besides, it is commonly accepted that goals of $80 \%$ pre-outfit at the section building stage are reasonable and achievable. This goal so far often remains beyond reach in one-off shipbuilding.

2. The boundary of types of assembly work.

- Four main categories of assembly work are elaborated in the research:

- Pipe installation-installing and welding pipes, including spools and pipe supports

- Electrical cable tray installation- installing and connecting electrical cable trays and pipes supporting lighting arrangements, including measuring, cutting and welding their supports

- HVAC ducts installation- Cutting, connecting, coupling insulated or uninsulated, spiral or rectangular ducts

- Equipment installation- Installing all kinds of equipment, like pumps, boilers, engines, generators, etc., which must be installed or can be installed during the section building and hull erection stage

The manufacture and assembly of piping systems represents one of the largest outfit tasks in shipbuilding. For specialized ships, although the number of pipe spools in the whole ship depends on the dimension and the type of that ship (for example, diving support vessels have more pipes than dredgers), it may easily reach 5,000 pieces. According to the report of a foreman from a piping company, around $85 \%$ of them are installed before the ship is launched. In addition, the main routes of cable trays, the total length of which can be between 2-4 kilometers, and pipes supporting lighting arrangements are installed during the pre-outfitting stage. Furthermore, complex vessels are heavily equipped for their size. Increased emphasis is being placed on early outfitting of sections before assembly to avoid installing equipment under cramped conditions later (Lamb, 2004). 
22 Automatic generation of assembly sequence for the planning of outfitting processes in shipbuilding

- Small iron work, paintings, insulation, joinery and scaffoldings are excluded from the research.

- Small iron work, like installing equipment foundations, doors, ladders, hatches, and windows, is not included. Most of them are portable items and their installation time is more flexible with less consideration of space accessibility. For example, it is no problem to install a water-tight door either during the section building stage or during the hull erection stage. This depends very much on its purchase lead time. Of course, earlier installation can save lots of effort. Yards often prefer to take the small iron work as part of the steel construction instead of the outfitting. If this is the case, it precedes outfitting. However, also co-makers carry out small iron work. Not all of this is done before (pre-)outfitting starts. Thus, while it is excluded in this report, ultimately our method will have to include all small iron work.

- As to painting, as long as the space in painting halls is available, steel sections, which have already been pre-outfitted to a certain degree, can be sent in and painted. This procedure does not involve any other assembly work and is not part of the typical pre-outfitting. Two types of painting take place during the outfitting on-board: final painting of compartments and the painting of the outside of the ship before the launching. The principle of assembly work on-board relevant for painting is to reduce the damage to the basic painting as much as possible and try to be finished before the final painting. The application of this principle varies across different types of assembly. For example, since cables are not allowed to be painted, before pulling cables, the electrical company will ask the painters to do the final painting of cable trays, steel strips and foundations of electrical equipment first. But for steel pipes, there is no such need. Detailed product data relative to paint (locations, layer, preconditions) and a detailed painting schedule is not normally available at present. To simplify the problem, we do not deal with the painting in the research.

- Insulation is excluded in the research for two reasons. First, detailed insulation drawings have not been integrated into the detailed 3D model. In a practical situation, it is not easy to access digital insulation information. Secondly, when insulation takes place, it is influenced by many factors. For example, insulation studs are welded or shot onto the steel plates by subcontractors before sections are sent to the slipway. Then on board, some $25 \%$ of the required insulation area (the ceiling, if necessary, and the upper parts of the walls) of a certain compartment has been covered with the insulation material before larger pieces of equipment are installed. The insulation of the lower parts usually is postponed to the last moment when the installation of all pieces of equipment is finished. This 
way it is tried to avoid damage of the insulation material by other installation work. Meanwhile, the insulated areas vary from compartment to compartment. A proper inclusion of insulation work would thus require comprehensive digital data on layout, component population, compartment particulars and insulation details. For reason of data scarcity, this aspect was excluded from the research.

- Joinery, which includes installing accommodation parts such as cabins or berths, dining facilities, food preparation areas, and rooms for meetings or other administrative purposes, is excluded from the research. The reason for this choice is that it can be done more or less separately and independently without interfering too much with the assembly of steel structure and other outfitting elements. Also it is a process that comes relatively late in the planning and is relevant only for a part of the compartments on board, mainly in the superstructure.

- Indeed, scaffoldings are required almost across all production stages. However, they are not a part of the ship. The purpose of scaffoldings is to provide a safe place of work with safe access suitable for the work (overhead welding, painting, cable pulling, etc.) being done. Thus they are regarded as a necessary resource and do not play a role in the research.

The exclusion of these aspects from this research does not mean that the importance of these works should be underrated. For example, the installation of a certain piece of equipment is carried out based on the prerequisite that its foundation, if it needs one, has already been welded on the steel structure. It is possible that to make sure the hull erection plan is fulfilled, painting halls may become one of the bottlenecks in production processes. And indeed, all these mentioned aspects do influence the total outfitting duration considerably that will be elaborated in chapter 9.2.2.

Once again, the four main categories of assembly work covered in this research play a significant role in the pre-outfitting and outfitting on-board stage, covering something like $75 \%$ of the total outfitting work estimated by an outfitting coordinator. Making the planning of the outfitting activities of these four categories helps to control the whole outfitting processes. Moreover, given the fact that only the technical aspects of assembly knowledge are considered, the interdependencies among these outfitting activities still remain valid and are not influenced by excluding other types of outfitting work. Thus, it is believed that this research provides an important contribution to dealing with the major part of the earlier outfitting (pre-outfitting and outfitting on-board) work in shipbuilding. 



\section{Chapter 3}

\section{Outfitting Planning}

We have seen that the intrinsic characteristics of outfitting processes to a certain degree pose challenges to improve assembly work efficiency in terms of many savings in man-hours and duration. This chapter deals with the possibility to tackle these challenges by introducing the idea of integral outfitting planning. Section 3.1 gives an overview of current outfitting planning as used in shipbuilding. Both the current practices and their strengths and weaknesses are described in section 3.2 with the purpose of providing the foundation for a novel approach to integral outfitting planning which will be illustrated in section 3.3. A summary is provided in section 3.4.

\subsection{An overview of outfitting planning in shipbuilding liter- ature}

Business planning has multi levels: strategic, tactical and operational (Galnur, 2005). This can also be applied to the production planning in shipbuilding processes as displayed in figure 3.1. The strategic planning is long term planning and involves making decisions which will work towards reaching a shipyard's vision. It starts before any established contract and covers all contracts, signed and anticipated. It defines the production program for a ship based on the early design with the aim to meet a contractual delivery date. During this stage, construction milestones are established. The tactical planning is the middle term planning which typically ranges from a couple of months to one year. It is used to detail the strategic planning on a more practical level. It states what will be produced and when it will be produced, considering the total demand on plant resources. Section building schedule, hull erection schedule and engineering schedule are set up during this stage. The operational planning and control is on a weekly basis. During this stage, e. g. the foreman on the shop floor allocates the resources that are available to him, realizes the plan and reacts to possible disturbances like missing materials or information, drawing modifications and machine breakdowns. The ideal outfitting plan is expected not only to be relevant 
26 Automatic generation of assembly sequence for the planning of outfitting processes in shipbuilding

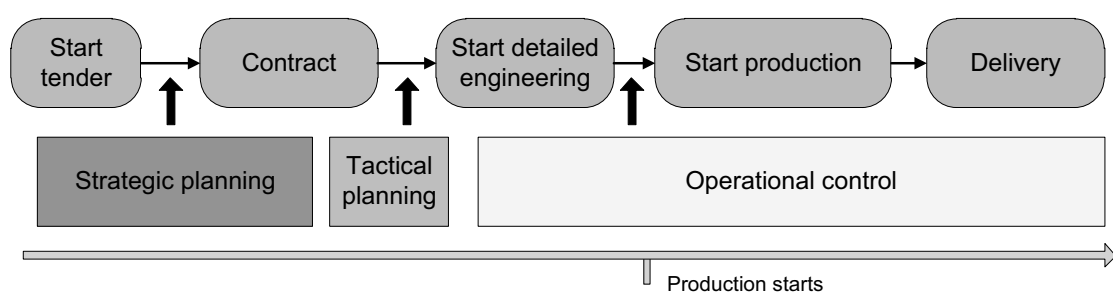

Figure 3.1: Phases of production planning

for meeting strategic and tactical plans but also to be instructional enough to help the operational control on the site.

Why is outfitting planning needed? Shipbuilding is primarily concerned with the assembly and construction processes. Management and control of such a large assembly operation is very complex, particularly because each ship is often a one-of-akind endeavor (Diesslin, 1982). Add to this the physical aspects of ship construction, large and inflexible material (e.g., steel plate, piping), outdoor construction, lack of standardization, limited work and storage space, etc., and it soon becomes obvious that anything which can simplify the construction process will be very useful in increasing productivity. Viewed simply, outfitting planning does this by breaking the work on the ship down into a series of small units (other than the whole piece of superstructure) or pallets instead of several large systems (piping, ventilation, etc.) or zones. These are much easier to control and actually increase the flexibility of management to meet deadlines, since many small, well-organized tasks are easier to change and/or reschedule than attempting to modify a few very large tasks (Diesslin, 1982). This is especially true since it is difficult for employees to keep track of all the interdependencies within or between large chunks of work.

The one-of-a-kind nature of many shipbuilding projects is a significant barrier to productivity improvement since it reduces the ability of labor to learn through repetition and makes the application of mass processing production concepts difficult to justify and apply. On the other side, there are many similar activities involved in shipbuilding almost regardless of the type of ship. Outfitting planning views each ship in units which are common to most ships. From there, the assembly work during the outfitting stages is differentiated into series of activities with similar work content in different combinations. The type of assembly, either pipe fitting or cable tray installation, will vary section by section, depending on the size and type of ship, but very often the work content, methods and production considerations do not change very much.

Outfitting planning should never be treated as a separate process as it has an im- 
portant relationship with the way that the other shipbuilding processes are proceeding. Before describing the current practice in outfitting planning, we first provide an overview of previous work on this issue even though, this topic has not received much attention in shipbuilding literature. These are three outfitting methods: conventional outfitting, zone outfitting and modular outfitting.

\section{Conventional outfitting}

Conventional outfitting, in contrast to 'Zone outfitting' described later, was planned and implemented along the lines of the function systems (Jonson and Chirillom, 1979). It was widely applied before the concept of 'Zone outfitting' was introduced in the late 1970s. It was typified by the allocation of resources to activities associated with ships' systems, e.g., cargo oil, bilge and ballast, main propulsion, etc., and did not recognize that certain interim products, i.e. subassemblies, could be produced more efficiently away from hull erection sites.

The assumption which a conventional planner usually made was that outfitting should commence in a given area as soon as that portion of the ship was erected (Jonson and Chirillom, 1979). The work was usually planned as a follow-on to hull construction. The sequencing of the outfitting effort was accomplished system-by-system with designated starts not earlier than completion of the surrounding hull. Thus the planned sequences and allocations for outfitting were not, with rare exceptions, optimal for minimizing resources.

\section{Zone outfitting}

In the late 1970s, the zone outfitting method was introduced with a purpose of doing more outfitting activities earlier in the production process. It recognizes that certain multi-system interim products, such as significant subassemblies of structural and/or outfit materials, can be produced more efficiently away from hull erection sites. This approach allows most of the outfitting work to be accomplished earlier and in shops where it is safer and more productive with higher quality. Outfitting, thus organized, is not a successor activity to hull construction, but is accomplished simultaneously with it, and hence is as much as possible independent from the large-scale hull erection progress.

Zone outfitting is divided into three basic stages listed by order of priority (Jonson and Chirillom, 1979), (Diesslin, 1982):

- On-Unit. The assembly of an interim product consisting of manufactured and purchased components not including any hull structure.

- On-Block. The installation of outfit components, which could include a unit, onto a hull structural assembly or block prior to its erection. 
28 Automatic generation of assembly sequence for the planning of outfitting processes in shipbuilding

- On-Board. Installation of any remaining outfit material and the connection of units and/or outfitted blocks.

Some outfit items can only be installed on-board, like furnishings. Some can be referred to as free components since they may be installed in any of the three modes, like doors. Thus, the full exploitation of the zone outfitting concept requires that production management is able to resolve all the alternative choices available.

Meanwhile, the hull block/section erection schedule is a constraint in outfitting planning because it obviously fits the date of ship launching which is one of the milestones in the production process. It is also possible to treat the hull block erection schedule as part of the decision process, i.e. if it were rationally justifiable, a hull block/section might remain in the assembly area longer to allow more pre-outfitting to be performed.

Another constraint which may affect outfitting planning decisions in many yards is the available lifting capacity. The weight of pre-outfitted blocks/sections must not exceed the safe lifting capacity of the available equipment. The effect of outfitting planning decisions on the yard's own resources must also be considered. Among the resources to be considered are labor and material availability and production or storage space.

Based on these constraints, a decision support system (DSS) was developed (McGinnis and Graves, 1980), (Graves and McGinnis, 1982). In developing such a model, two types of decisions were identified: selection decisions and scheduling decisions.

It will be convenient to formalize the selection decisions first. Associate with each outfit component an index, $\mathrm{i}$, where $\mathrm{i}=1,2, \ldots, \mathrm{N}, \mathrm{N}$ being the total number of outfit components. Similarly, associate with each outfit unit an index $\mathrm{j}=1, \ldots, \mathrm{M}$, and with each block an index $\mathrm{b}=1, \ldots, \mathrm{B}$.

The selection decisions are represented by an indicator variable. For a particular component $\mathrm{i}$, the variables are:

$$
\begin{aligned}
& x_{i}^{u}= \begin{cases}1 & \text { if component } i \text { is outfitted on - unit } \\
0 & \text { otherwise }\end{cases} \\
& x_{i}^{b}= \begin{cases}1 & \text { if component } i \text { is outfitted on - block } \\
0 & \text { otherwise }\end{cases} \\
& x_{i}^{h}= \begin{cases}1 & \text { if component } i \text { is outfitted on - board (in the hull) } \\
0 & \text { otherwise }\end{cases}
\end{aligned}
$$


There are similar indicator variables associated with each unit:

$$
\begin{aligned}
& z_{j}^{s}= \begin{cases}1 & \text { if unit } j \text { is installed on-section assembly } \\
0 & \text { otherwise }\end{cases} \\
& y_{j}^{b}= \begin{cases}1 & \text { if unit } j \text { is installed on - block } \\
0 & \text { otherwise }\end{cases} \\
& y_{j}^{h}= \begin{cases}1 & \text { if unit } j \text { is installed on - board (in the hull) } \\
0 & \text { otherwise }\end{cases}
\end{aligned}
$$

and again similar indicator for each block. In addition, when making selection decisions, the precedence relationships defined by sequencing requirements must also be satisfied. Define:

$\mathrm{P}(\mathrm{j})=$ index set of components (units) which must precede component $\mathrm{j}$ (unit $\mathrm{k}$, when considering the unit assembly process) in production.

Then the precedence constraints on the selection decisions are:

$$
\begin{aligned}
& x_{i}^{u}+2 x_{i}^{b}+3 x_{i}^{h}-\left(x_{j}^{u}+2 x_{j}^{b}+3 x_{j}^{h}\right) \leq 0(i \in P(j)) \\
& y_{j}^{b}+2 y_{j}^{h}-\left(y_{k}^{b}+2 y_{k}^{h}\right) \leq 0(j \in P(k))
\end{aligned}
$$

to make sure that e.g. an on-block component is not mounted before an onunit component. These constraints require that for any component or unit, its predecessors must be outfitted or installed at the same time or during an earlier production stage.

In order to deal with the time and labor availability constraints, the scheduling decisions must be formalized. The following scheduling variables are defined:

$t_{i}=$ scheduled start time for component $i$ outfitting (on unit, on block or in the hull)

$\theta_{j}=$ scheduled time for completing unit $j$ fabrication

$\tau_{j}=$ scheduled start time for unit $j$ installation (on block or in the hull)

$d_{i}^{u}=$ time to outfit component $i$ on unit

The scheduling variables must satisfy all the precedence constraints as well as the scheduling limitations imposed by the steel schedule.

Consider the constraints involving on-unit outfitting.

$$
t_{i} x_{i}^{u}+d_{i}^{u} x_{i}^{u}-t_{j} x_{j}^{u} \leq 0(i \in P(j))
$$

This constraint requires that all predecessors of component $\mathrm{j}$ must be completed before component $\mathrm{j}$ can be outfitted on-unit.

$$
t_{i} x_{i}^{u}+d_{i}^{u} x_{i}^{u}-\theta_{j} z_{j} \leq 0(i \in P(j))
$$


30 Automatic generation of assembly sequence for the planning of outfitting processes in shipbuilding

The above constraint requires all on-unit outfitting to be completed before the unit itself is complete.

Similarly, other constraints are also set up for on-block outfitting. The final outfitting planning should satisfy all constraints required by selection decisions and scheduling decisions. The model requires large amounts of information , which was to be obtained from an outfit planner's experience, and generates large numbers of detailed decisions. Thus, any practical implementation will require a fairly detailed, production orientated data base to support the solution procedure (Graves and McGinnis, 1982). However, since many shipyards did not have such a data base at that time and were not willing to build it, the use of the DSS system as a support tool for the outfitting planning according to the concept of the zone outfitting was not widespread.

\section{Modular outfitting}

The concept of modular construction is not new in the manufacturing, construction, automotive, aeronautical or marine industries (Baade et al., 1997). Modular outfitting in shipbuilding is to assemble the equipment and other outfitting components in the work shop. Such assembly takes place concurrently with steel structure construction and should be easily lifted without exceeding crane-lifting capacities during the installation. The biggest advantage of modularization is the separation of the construction area and time between shipbuilding and outfitting activities (Altic et al., 2003).

It is very important that early in the project phase it is determined what areas of the ship can be modularized. It begins in the early stage of design, especially in machinery arrangement. At this stage, functionally related equipment, systems and tanks are located in order to reduce the occupied area and maximize the potential of modularization and standardization. The final module content and layout is confirmed by a series of studies, build strategy, and preliminary system routing. Thus, modules are optimized based upon engineering, spatial, regulatory, and economic parameters (Asok and Aoyama, 2009; Altic et al., 2003; Rubesa, 2011).

Thyssen Nordseewerke in Emden (Germany) developed a patented concept for modular construction of its engine rooms (Baade et al., 1997). It allows mutually independent construction of the ship structure and the engine room module. Nevertheless, there have been a number of problems. Two of the most pronounced problems are due to cost increases. The cost of design increased as a result of the level of detail required for production. The production costs also increased due to the requirement for a new production factory and the transportation equipment required to move the engine room to the construction site. But there are more problems to be expected. Such modularization will 
normally require extra space on board since systems may no longer efficiently share the same space. Also introducing an additional grouping structure (module) will oppose other groupings, reducing some of the advantages.

Along with the development of hardware and software technology, another two concepts have been proposed in the last decade. These are digital manufacturing and constraint-based simulation of outfitting processes. They may support outfitting planning and outfitting work in the future.

\section{Digital manufacturing}

Digital manufacturing is essentially a computer technique for defining all the steps necessary to build a product (structural assembly, install an engine, join blocks together, bend pipe, etc.), test them for completeness, and generate machine and work instructions to manufacture/assemble them (Popko and Barlach, 2003). The overall phases are: process planning; process detailing and validation; resource modeling and simulation; the extraction of manufacturing data and work instruction. It deals with both Design-for Manufacturing and Design-for-Assembly. It is used to determine the optimal production plan, taking into consideration real-world constraints. The application of digital manufacturing technology in shipyards was put forward and published by DELMIA Corp (Popko and Barlach, 2003) at the beginning of the 21st century.

It claims to be able to provide digital manufacturing and simulation support from concept design through manufacturing execution. It increases the productivity by using the 3D model-based environment for Design-for-Manufacturing and Design-for-Assembly activities. It also maintains its ability to analyze 'what-if?' scenarios and understand dynamic relationships between design, layout and production systems behavior. One disadvantage is that such software is prohibitively expensive. Also it requires extremely complicated algorithms, which often are not available. Indeed, this research develops some of the needed algorithms. Currently this development focuses more on the visualization of the 3D model, which scarcely refers to how to carry out the outfitting assembly work.

2. Constraint-based simulation of outfitting processes

Simulation has been established as an effective tool to support facility layout planning and production planning on shipyards. Some initial research results (van Alphen et al., 2004; Kaarsemaker and Nienhuis, 2006; Steinhauer, 2006). Konig (Konig et al., 2007) proposed a constraint-based simulation approach to detail outfitting tasks and their corresponding restrictions and requirements. Typical outfitting restrictions and requirements are specified as hard and soft constraints. For example, technological dependencies, capacity, availability of 
32 Automatic generation of assembly sequence for the planning of outfitting processes in shipbuilding

material and safety criteria are classified as hard constraints; productivity and outfitting strategies are regarded as soft constraints.

By using the simulation models, different assembly strategies were analyzed regarding the required execution time, worker's efficiency and space utilization. However, possibly the most prominent hard constraints, the technological dependencies between outfitting activities, were derived manually, which implies a prohibitively labor-intensive process.

\section{Summary}

Basically in practice, detailed planning of outfitting processes is not in any useful detail covered in the shipbuilding literature. Although this has not become a main factor to stop many ships from being launched on time, shipbuilders may suffer from escalating costs, long lead times, and schedule delays. This research concentrates on the development of outfitting planning in shipbuilding. Therefore, before we look at how to make such a detailed outfitting plan, the following section provides a closer look at the current way for shipyards to plan the outfitting work.

\subsection{Current shipbuilding practice}

When making the production plans, each shipyard has its own way and resorts to its own experience. This is because of availability of production plans from the past, the facilities of the yard, the type of vessels being built, the way planners prefer to work, etc. These together determine the way of generating the production plans. However, for a specific shipyard, their planning process is fairly fixed apart from minor projectspecific changes (such as an extra equipment delivery plan because of specialization of a certain ship). For example, before pre-outfitting a section, as a rule of thumb, there may be reserved 8 weeks for the material preparation and 4 weeks for the steel parts fabrication and assembly of that section.

The example shown in figure 3.2 illustrates how a modern shipyard, specialized in building offshore vessels, generates the production plans. Basically, it adopts the forward and backward planning method. Since the slipway work is leading ${ }^{1}$, the planners start from the hull erection stage. Aiming at meeting the milestones stated in the contract, they extrapolate the section building plan, pre-outfitting plan, hull erection plan and equipment delivery plan. These production plans will be distributed to the participating companies.

Having taken consideration of how complex each steel section is, the pre-outfitting plan states how many weeks are assigned to a certain section. The execution of the pre-outfitting plan is normally considered more flexible than that of the hull erection plan. Even if a delay in section construction causes only little pre-outfitting work to be done in that section, the date for that section to be put on the slipway is not

\footnotetext{
${ }^{1}$ The slipway is an asset of strategic importance to a shipyard in its long-term production plan
} 


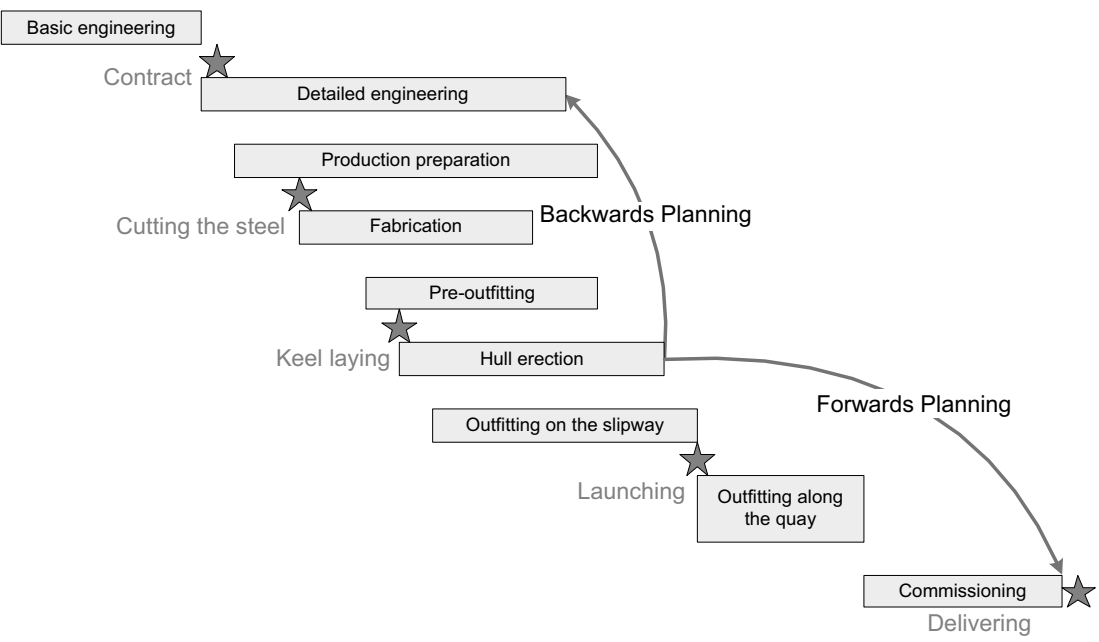

Figure 3.2: How to make the production planning

normally rescheduled. The reason is that to reschedule one section erection date may influence the erection dates of its neighbor sections and finally cause the delay of ship launching. This is however one of the crucial milestones on which a shipyard often receives a certain payment from a ship owner. Also changing the launch date immediately affect the construction schedule for the next ship. In order to determine how to carry out assembly work on a specific section and to cope with unexpected disturbances, the yard organizes team meetings every other week, in which all involved companies participate. In these meetings with the yard playing the role of coordinator, encountered problems are discussed, work progress is reported and new weekly plans are fixed. The importance of the team meetings for creating daily production plans for medium-sized yards cannot be overemphasized (Dlugokecki et al., 2009). It gives the benefits of transparency of the process and a clear insight into interdependencies, and is the basis for production control. The discipline of all participating companies in joining team meetings and creating even daily plans is a necessary step to sustain the implementation of both the pre-outfitting and outfitting plan.

Nevertheless, the rough pre-outfitting and outfitting plan plus team meetings are far from sufficient to cope with unexpected interferences on a daily basis. The number of possible execution solutions is very large though limited by technical constraints. These are, for example, constructional dependencies between the executed work steps, the limitations of available employees and equipment and the availability of working space to execute a given work step. If sufficient working space is not available, workers' productivity will be reduced, and as a consequence projected execution time and costs will rise. Thus, extensive and integral planning, which takes 
34 Automatic generation of assembly sequence for the planning of outfitting processes in shipbuilding

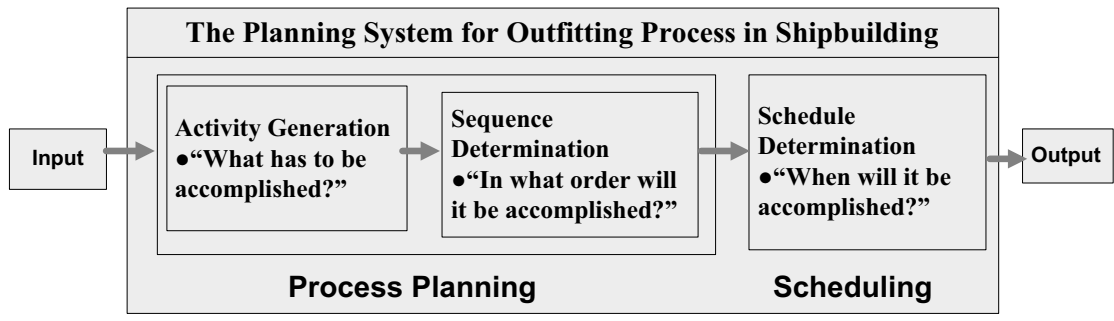

Figure 3.3: Planning system for outfitting processes

into consideration all possible technical attributes and constraints that have a dominant influence on the carrying out of assembly work in steel structures, is still needed. Currently, such integral (outfitting) planning is not normally available.

\subsection{Integral outfitting planning}

\subsubsection{What is integral outfitting planning?}

Integral outfitting planning means the organizational process of creating and maintaining a plan for outfitting processes by the participating companies providing insight into each other's contribution to the outfitting processes and the effects of choices made in those processes. The idea of integral outfitting planning originates from the outsourcing strategy nowadays pursued by all companies. The distribution of activities across actors necessitates improving the coordination among co-makers. It aims at communicating better about each co-maker's activities in advance, thus reducing the conflicts on the work site. In our research we put forward a planning system for this goal.

In Figure 3.3 apart from the steps of input and output, the planning system for the outfitting process, on the operational level, has been divided into three interrelated steps: activity generation, sequence determination and schedule determination, trying to answer questions "What has to be accomplished?", "In what order will it be accomplished?", and "When will it be accomplished?" respectively. The first two steps comprise process planning and the last is scheduling.

This planning system comprises of the following five steps:

1. Input

The basic input is the detailed engineering CAD model which is provided by different co-working organizations (yard and different sub-contractors, sometimes by the customer in case of owner's deliveries) and integrated during the engineering processes. 


\section{Activity generation}

The activity generation method is used to automatically translate the CAD model information into a list of assembly activities. Currently, such a list is not adopted by the shipyard or subcontractors. Today after getting the information from foremen, such as 2D drawings and specification sketches, workers start their installation work according to their experience. In our proposed planning system an activity template, representing the typical installation work, is adopted to generate the activity list.

The activities in the template were recorded during the observation on the work floor. They are classified and arranged as follows:

- Preparation work. Make sure drawings, tools, material and personnel are all available before starting installation.

- Measurement, i.e. precisely find the positions of the component with its supports in the steel structure.

- Connection. If the component is the first piece to be installed in a section, this step may be omitted. Otherwise, the connection of the current piece to the formerly installed one is needed. Meanwhile, a heavier item requires the activity of using a crane in this step. In a large space some form of scaffolding may be necessary.

- Support-making. Since all information about the support is currently not included in the drawings, this step comprises the whole support-making process, including calculating their length, cutting, grinding, welding and also picking up corresponding bolts and nuts.

- Component-mounting. This step concerns the actual work to put the component in the steel structure. For electrical cable trays, supports may first be welded on the tray and then the whole piece will be welded to the steel structure; a ventilation duct may directly be put on the supports which have been already welded in the steel structure; supports for a lighter pipe may be inserted between the pipe and the steel structure because the pipe has already been connected with the valve, reducer or formerly installed item and the space between the pipe and steel plate is rather limited.

These five types of activity are not always carried out in a sequential process. Note that this template is in principle applicable for the installation of pipes, HVAC ducts and cable trays.

In the activity generation template, since the procedures of assembling spools bears many similarities, and to avoid excessive detail in the resulting sequences, we have grouped the activities on the spool level. Thus, the assembly of a certain spool, which can be a pipe spool or spiro-duct or cable tray, is regarded as 'one activity'. The duration of such 'one activity' is the aggregation of that of 
36 Automatic generation of assembly sequence for the planning of outfitting processes in shipbuilding

its sub-activities allowing for possible parallel execution of these sub-activities. This grouping simplifies the sequence determination and allows to mainly deal with how the attributes of each spool influence its mounting sequence.

3. Sequence determination

This step is to determine in what order the components, defined in the list, are carried out. Its focus is to detect the technical dependencies (explained in section 1.3) between components and their activities and to provide the assembly sequences.

4. Schedule determination

Once the assembly sequence of the components and their defined activities is known, the schedule determination carries out the resource allocation: the number of workers and the type of equipment. It aims to maximize the efficiency of the operation and thus reduce the costs and/or duration.

The two steps, activity generation and sequence determination, comprise process planning. Process planning is the bridge between the product design and manufacturing, and determines what tasks to perform and in what sequence. It emphasizes the technological and organizational requirements of a task.

Scheduling, the following step, receives process plans as the input and imposes time ordering and resources allocation constraints while satisfying the precedence relations given in process plans (Berry, 1990). The objective of scheduling is to balance work with required and available production facilities. A good schedule must reflect a satisfactory compromise among these competing influences (Liu, 1993).

Scheduling has received considerable attention and many methods and tools have been developed, like Mars Planning, Microsoft Project, Primavera, etc. This research focuses on process planning.

\section{Output}

The output is the feasible assembly sequence satisfying the time and resource constraints. From input to output, this is an automatic process with the integration of different disciplines.

Based on the above, the focus of our integral outfitting planning research is to determine the assembly sequence of each outfitting component. This brings us to the investigation of the subject of assembly sequence planning. In this area, much work has been done since the early 1980s in the mechanical engineering industry. Methods have been developed to automatically generate assembling procedures since 1977. Even now, assembly sequence planning, a critical technology that bridges the divide between product design and realization ( $\mathrm{Su}, 2008)$, is still an active topic in current researches concerning computer aided process planning (CAPP) . Whether 
this concept can be applied to the outfitting processes in shipbuilding and how it may function in practice, will be investigated in detail in chapter 4 .

\subsubsection{Adoption issues}

The full implementation of such a planning methodology, if and when possible, brings forth the following requirements to all co-makers.

- With the application of rules of marketing game, which is to 'cope with constantly changing situations by sticking to a fundamental principle-be willing to change' according to McConnell (McConnell, 2006), the yard should be willing to adapt its current way of planning to the new planning methodology.

Since the yard today plays the role of coordinator of the project, its emphasis should be placed not only on getting the ship built, with little regard for the efficiency of all parties involved, but also on motivating subcontractors to supervise performance. The yard should take the initiative to encourage the involved parties to use this or indeed any new planning methodology. It requires a long-term commitment to realize and complete this methodology on the operational level, through organizing the necessary trainings, through offering co-makers benefits resulting from the application of this methodology, etc.. This is justifiable because the yard stands out most to gain in terms of either profit or its reputation in the field.

- All parties who are involved in the engineering processes should make efforts to generate more and more consistent information in that phase. The more detailed the product model, the more precise the results generated by the planning method are, and the more probable for the production plans to be stuck to.

- All involved parties should be willing to use this planning method at the same time and cooperate with each other to monitor and execute the outfitting work according to the pre-defined assembly sequence. Their outlook should be broadened to the whole project when making decisions. It may require them to put prejudices aside which may be caused by all kinds of reasons, like personal preferences or different work styles, and run the project together toward the same goal.

- Last but not the least, all parties should keep an open mind to challenge what they are doing now. Doing something for many years does not mean this is the best or even the correct way to do it. Quite often, routines blind people and make them ignorant of the potential of change. This happens more often to workers, the downstream of the production line, who decide to a large extent if the new planning tool can really be applied on the work site. Getting workers trained should start by helping them to depend less on their deep-rooted routines and be more open to alternative work approaches. 
38 Automatic generation of assembly sequence for the planning of outfitting processes in shipbuilding

Just as every coin has two sides, this planning system is no exception. Currently, the development of this method is in the research stage, which will be elaborated in detail in chapter 8 and 9 . To apply it in the shipbuilding industry operationally, at least the following requirements need to be considered.

- Ability to access to companies' databases. So far, the input of this system is specifically prepared for the research purpose. It is not general enough to allow all co-makers to access their own databases in an automated manner.

- Additional testing to debug the system. The test cases in the research are not sufficient to make sure the software is flawless.

- Adding a professional user interface. The current interface is too abstract and only suitable for academic purposes.

The functional limitations of the system will also be described in the later chapters.

\subsection{Summary}

This chapter introduces different ways to carry out outfitting planning. From the conventional outfitting before 1970 to the constraint-based simulation of the outfitting process in the first decade of 21 st century, the outfitting planning has developed correspondingly. It has moved from the allocation of resources to activities associated with ships' systems to being evaluated beforehand by the use of simulation. Against the background of the strategy of outsourcing, the current outfitting planning on the tactical level is done by shipyards and the detailed planning on the operational level is executed by individual co-making companies under the coordination of the yard.

Although the topic of how to develop a more efficient and reliable outfitting planning system has not received sufficient attention due to either its intrinsic complicated nature or people's unconscious tendency to ignore it, the idea of integral outfitting planning is vital for improved shipbuilding operations. Thus, a new planning system has been put forth. Apart from the steps of input and output, it consists of three interrelated steps:

- Activity generation

- Sequence determination

- Schedule determination

The implementation of this planning methodology poses challenges to all participating companies. An open mind and the willingness to objectively coordinate activities are a few of the prerequisites. 
The focus of this research is the sequence determination. The assembly sequence determination has been tackled in the mechanical industry. To see if the same approaches can be applied in outfitting work and to what degree it works will be further analyzed in the following chapter. 



\section{Chapter 4}

\section{Assembly Sequence Planning Approaches}

This chapter deals with the methods used to generate assembly sequences in the mechanical industry, and the possibility of their application in shipbuilding outfitting processes. These methods include liaison diagram, a tree structure, AND/OR graph and the binary matrix method. Section 4.1 gives a brief introduction of what assembly sequence planning entails. The similarities between the assembly of mechanical parts and that of outfitting components in a ship section are also illustrated here. Section 4.2 provides a short overview of the methods developed in the last 30 years to generate the assembly sequence. The advantages and disadvantages of each method, and its applicability for the purpose of this research are analyzed in detail. Section 4.3 summarizes conclusions.

\subsection{Introduction of assembly sequence planning}

\subsubsection{General introduction}

Specific terminology is used in the mechanical industry. Familiarity with them helps understand why and how the assembly sequence planning may be applied in the shipbuilding industry as will be explained later in this chapter. The terms that need explanation are as follows:

- Assembly

Assembly is a very important activity for manufacturing enterprises. Wang and $\mathrm{Li}$ (Wang and $\mathrm{Li}, 1997$ ) defined assembly as a 'process of creating a connection between components or subassemblies to form complex products'. Nof et al. (Nof et al., 1997) defined assembly as 'the aggregation of all processes by which various components and subassemblies are built together to form a 
42 Automatic generation of assembly sequence for the planning of outfitting processes in shipbuilding

complete, geometrically designed assembly or product (such as a machine or electronic circuit) either by an individual, batch or a continuous process'.

Note that the word can be used as an activity (to assemble) and as a product (assembled things).

- Component

A component is a basic part which, at least on the site where the assembly takes place, is not assembled itself from smaller parts. So, a switchboard may be the result of an assembly operation at the electrical company, but it is a component for the assembly process at the yard.

- Subassembly

A subassembly is a group of assembled components built for certain functional or manufacturing purposes. A subassembly is less complete than an assembly. An assembly could also be composed of several subassemblies. The distinction between assembly and subassembly is vague and depends on the perspective. A steel section which has been pre-outfitted can be regarded as an assembly when compared to a single component. It also can be considered as a subassembly when compared to a whole ship.

- Assembly sequence

We know each component is a distinct, solid object, of which the shape in most cases remains unchanged (unless it contains internal joints, e.g. hinges or if it is flexible such as a cable). Components are interconnected whenever they have one or more compatible surfaces in contact. Surface contacts between parts reduce the degrees of freedom for relative motion (Homem de Mello and Sanderson, 1991a). Because of geometrical and physical constraints among different components of an assembly, certain components must be assembled before assembling others. Even if there is no such hard rule, there may be organizational reasons to adhere to a certain order. This ordering among different components is called assembly sequence (Huang and Lee, 1989).

- Assembly sequence planning

Most mechanical products can be assembled in several ways (Ben-Arieh, 1994), meaning that different sequences of assembly operations can result in the same final product. Each such sequence implies different a degree of difficulty for the various operations. Thus, the main purpose of assembly sequence planning is to organize a proper assembly sequence with which the components can be grouped or fixed together to construct a final product (Tseng et al., 2007).

We use an example to further illustrate the above terms. The product to be assembled consists of four parts labeled cap, stick, receptacle, and handle as shown in 


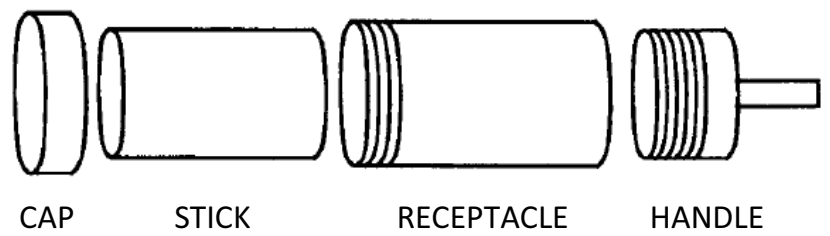

Figure 4.1: A simple product in exploded view (reproduced from Homem (Homem de Mello and Sanderson, 1990))

figure 4.1. The stick is a solid cylinder which fits inside of the receptacle, and the receptacle is a hollow cylinder which is closed on one end by the cap and on the other end by the handle. Figure 4.2 shows one of its corresponding assembly sequences. It can be seen that the component stick, receptacle and handle are joined together first to become a subassembly. The assembly sequence of figure 4.2 also shows that the subassembly is built by combining the handle and receptacle first, then joining the stick. The cap is finally attached to the rest.

\subsubsection{A proper assembly sequence}

In general, there are two types of constraints to evaluate whether the assembly sequence is proper or not, one is the technical constraints, dealing with the feasibility, and the other is the business constraints, checking the reasonability in organizational and financial terms.

\section{Technical constraints}

- Collision/interference free path- There must be no collisions/interferences as each component is brought into contact with the rest of the assembly, either between components or between components and/or tools used during the assembly process.

- Accessibility- Each component in the assembly will be held in place either by surrounding components or by some joints to some foundations. In outfitting, it means that the work space and the corridors leading towards it must be sufficient for the component to be accessed by workers and their tools during the installation process.

- Stability- Every component must remain gravitationally and elastically stable at all times during and after the assembly. It means that the component maintains its relative position and (elastic) shape under the action of gravity, the action of assembly forces and any subsequent and reasonably expected influences such as weather or accidental loads. 
44 Automatic generation of assembly sequence for the planning of outfitting processes in shipbuilding

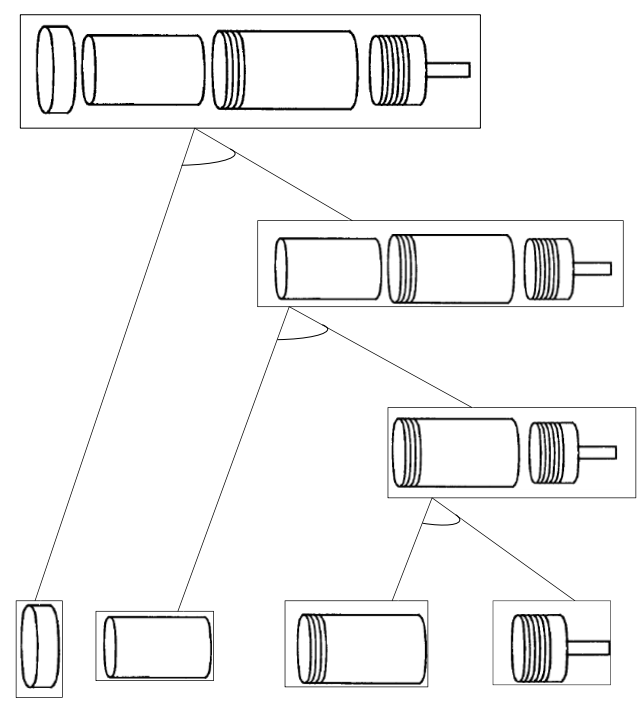

Figure 4.2: Example of an assembly sequence (reproduced from Homem (Homem de Mello and Sanderson, 1990))

\section{Business constraints}

- Assembly time- The assembly time of the product must satisfy accepted standards. If it takes too long, the sequence must be rejected.

- Resource utilization- The execution of the different work steps/assembly operations require various skilled employees and/or equipment. The sequence of these operations should utilize required resources efficiently.

- Cost- The assembly cost relates to assembly operations, assembly motions and assembly directions (Sharma et al., 2008).

A feasible assembly sequence must fulfill all the technical constraints. However, a proper assembly sequence should not only satisfy all the technical constraints but also violate the business constraints as little as possible. A proper assembly sequence might not be the best one, because the criteria used to judge the 'best' are quite subjective, but it is the sequence that meets the needs for a particular purpose within the available resources. As described in chapter 1.3, the outfitting assembly sequence to be tackled in our research is supposed to mainly fulfill the technical constraints. This will be further discussed in chapter 9.5 . 


\subsubsection{Similarities and differences}

Assembly sequence planning has drawn much attention of manufacturers and researchers since the 1980s. For industrial products, it is very important for a couple of reasons. On the one hand it provides feedback to the designer at an early stage, indicating how his product is suited to be assembled. On the other hand, assembly sequence planning guides automatic or manual assembly.

Before answering the question of whether the technology of industrial 'assembly sequence planning' can be applied in outfitting processes in shipbuilding or not, we take a closer look at the similarities and differences between assembling manufactured products and installing all kinds of components inside a ship's steel section during the outfitting stages.

\section{Similarities}

Similarities between assembly processes in the manufacturing industry and outfitting processes in the shipbuilding industry do exist. They both start with a separate and independent component and end with the final product which actually is the assembly of all these independent components. They both also require a series of assembly operations that put all these components together to become the designed final product.

Assembly plans in both industries are ordered sequences of operations that transform one configuration of components into another, starting with all components disconnected from each other and ending with all components properly joined to form the desired assembly. Therefore, it seems possible to apply the methods of assembly sequence planning in the manufacturing industry to the outfitting processes of shipbuilding industry.

\section{Differences}

The difference is caused by the nature of the 'final product'. An industrial product consists of a number of components. These components have physical contacts with each other because of the mating with others. When a component is joined to the already assembled components/ subassembly, it has a physical contact with at least one component. The way of how one component connects to the other influences the assembly sequence. For example, in figure 4.3, a caster consists of a shaft, spacer, fork, wheel and axle. The spacer has both the axial contact with the shaft and the surface contact with the fork. The shaft has the axial contact with both the spacer and the fork. In this case, if the shaft and the fork are connected first, there is no way to insert the spacer between them. Indirectly the wheel is connected to the shaft by the intermediate fork and axle. The size, tolerances, orientation and types of contacts all influence the assembly operations (Maziero et al., 2004).

However, in ship's outfitting, many components do not have (direct or indirect) contacts with others because components in different systems are largely independent from each other. As shown in figure 4.4, two pipe spools in different pipe lines are 
46 Automatic generation of assembly sequence for the planning of outfitting processes in shipbuilding

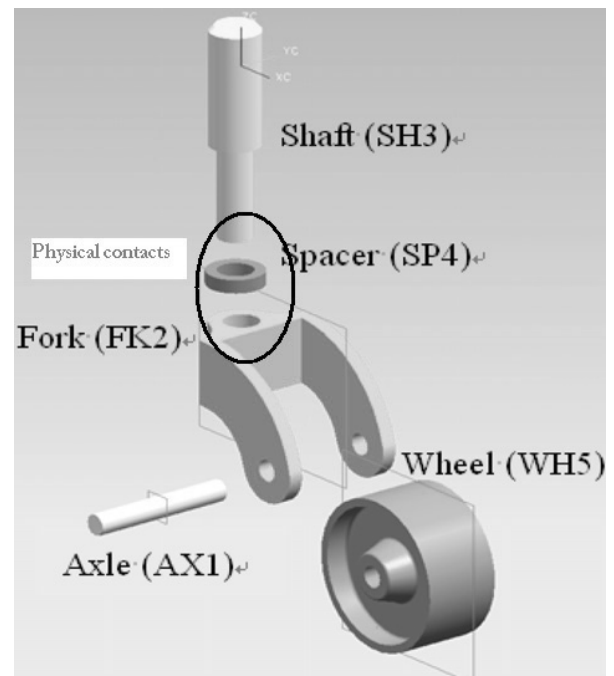

Figure 4.3: Physical contacts among components of a caster

separate. Even if the components (two pieces of spiro-duct for example) in the same system are connected end to end, they are not mated and their assembly orders would not be restricted by such physical contacts. Thus, the assembly sequence of outfitting components is much less influenced by such kind of physical contacts.

\subsection{Automatic generation of assembly sequences-a litera- ture review}

Much work has been done since the early 1980s on assembly sequence generation. These researches focused on sequence generation and sequence representation. For sequence generation, there are two approaches- assembly and disassembly. 'Assembly' has already been described in the previous section. Let us take a brief look at disassembly.

Automatic assembly may be studied from the point of view of disassembly. As Woo described (Woo, 1987), 'If we visualize the process of assembly as having a finite number of 'states', each being captured by a single frame on film, then rolling the film backward corresponds to disassembly. The process of assembly/disassembly is reversible, if the components are rigid and there is no internal energy stored. (Such would not be the case with components of variable geometry such as spring). Therefore, the problem of establishing how to assemble a given product can be converted into an equivalent problem of finding how the same product can be disassembled.

Widely used and well-documented examples of assembly and disassembly methods that may be candidates to generate the assembly sequence in outfitting processes 


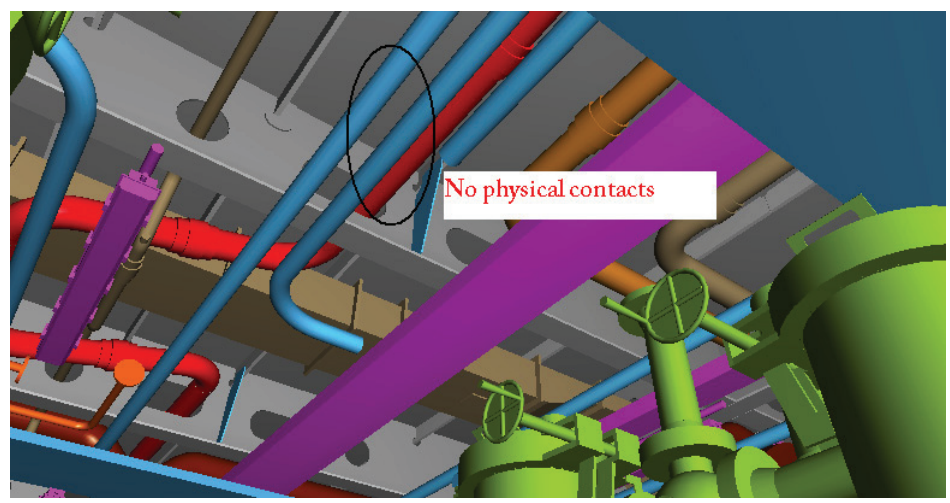

Figure 4.4: No physical contacts between two pipe spools in a section

are described next.

\subsubsection{The assembly sequence approach}

\section{Liaison diagram}

From the point of view of assembling the product, in 1984, Bourjault (Bourjault, 1984), (De Fazio and Whitney, 1987) presented means for generating all assembly sequences algorithmically from a series of rules. The rules are generated from the answers to a series of questions about the mating of parts.

He began with the liaisons diagram (the 'graphe de liaisons fonctionelles'), which is a graph devised to represent an assembly. The graph uses the nodes to represent the components of the assembly and the arcs to establish the relations between the components. Based on this diagram, a list of 'Yes-No' questions are generated, and by answering these question the assembly sequences is determined.

We use an example of assembling 7 components (6 pipe spools and 1 piece of cable tray) in a section as shown in figure 4.5 to illustrate this method. The liaison diagram manually generated by a practitioner is the first creative step in generating the family of assembly sequences. As shown in figure 4.6, the diagram is straightforward.

One may well note that the liaisons between pipe a and c, pipe a and d, pipe a and cable tray e are omitted. Most would agree that it is physically possible to install them ( $a$ and c, a and d, a and e) at the same time. However, such assemblies represent poor practice because they block the way to install the components located in-between them (along $\mathrm{Z}$ direction). The omission of liaisons between pipe $\mathrm{f}$ and $\mathrm{c} / \mathrm{d}$, and pipe $\mathrm{g}$ and $\mathrm{c} / \mathrm{d}$ prevents an assembly involving inserting pipe $\mathrm{b}$ between them. Thus, to generate the diagram requires the practitioner to be able to anticipate some possible sequences. It thus depends on the work experience and requires human labor. 
48 Automatic generation of assembly sequence for the planning of outfitting processes in shipbuilding

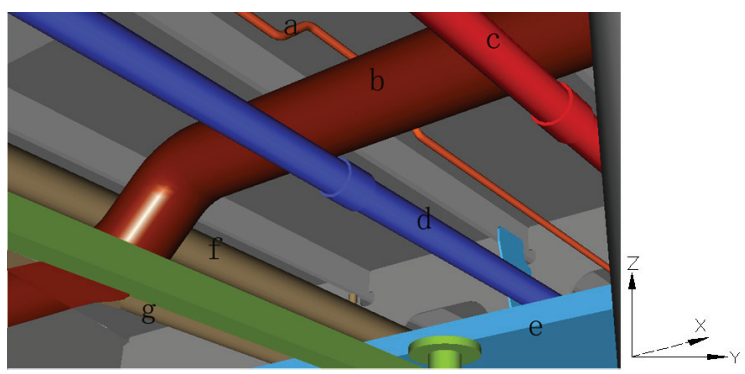

Figure 4.5: An example of assembly consisting of 7 components

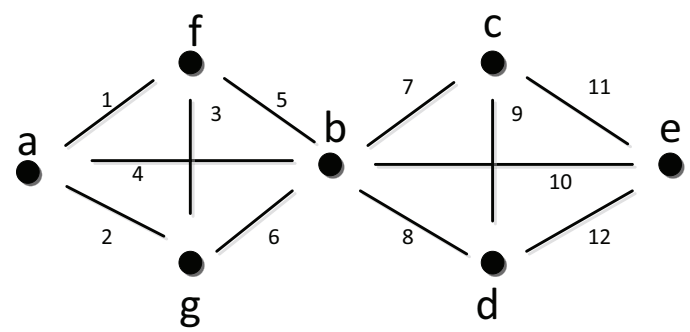

Figure 4.6: Liaison diagram of 7 components

The liaison diagram accomplished, the next process step is to ask and answer a group of questions. Bourjault's question is 'Is it true that $\mathrm{L}_{i}$ cannot be done after $\mathrm{L}_{j}$ and $\mathrm{L}_{k}$ have been done?' Here, $\mathrm{L}_{i}$ means the Liaison $\mathrm{i}$. Liaison means the mating of two parts, i.e. parts that have been manufactured to fit. In the outfitting problem, the mating of two pipe spools or ducts scarcely exist, apart from some penetrating pieces. Thus, our question becomes: 'Is it possible to install two components related by Liaison i at the same time?'. In case the answer is 'No', a second question needs to be asked: 'If it is not, which component related by Liaison i is first to be installed?'

$\mathrm{i}=1$ : Yes, pipe a and $\mathrm{f}$ can be installed at the same time;

$\mathrm{i}=2$ : Yes, pipe a and $\mathrm{g}$ can be installed at the same time;

$\mathrm{i}=3$ : Yes, pipe $\mathrm{f}$ and $\mathrm{g}$ can be installed at the same time;

$i=4$ : No, the installation of pipe a precedes pipe $b$;

$i=5$ : No, the installation of pipe $f$ precedes pipe $b$;

$i=6$ : No, the installation of pipe $g$ precedes pipe $b$;

$i=7$ : No, the installation of pipe $b$ precedes pipe $c$;

$i=8$ : No, the installation of pipe $b$ precedes pipe $d$;

$i=9$ : Yes, pipe e and d can be installed at the same time;

$i=10$ : No, the installation of pipe $b$ precedes cable tray e;

$i=11$ : No, the installation of pipe c precedes cable tray e; 


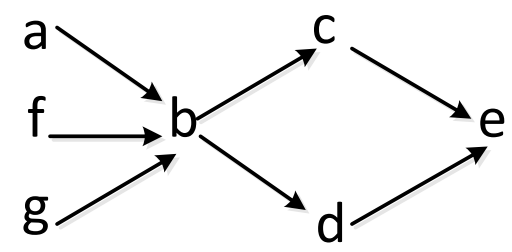

Figure 4.7: The assembly sequence of 7 components based on the liaison diagram

$i=12$ : No, the installation of pipe d precedes cable tray e.

The final assembly sequence is displayed in figure 4.7. Pipe a, $\mathrm{f}$ and $\mathrm{g}$ can be assembled simultaneously and are followed by the installation of pipe b. Then, pipe $\mathrm{c}$ and $\mathrm{d}$ are mounted. Cable tray e is the final one to be mounted because all the other 6 pipe pools are above it.

If the number of components increases, the application of Bourjault's method in outfitting processes seems not practical for two reasons. First, it is not easy for the practitioner to first generate a reasonable liaison diagram with numerous components and liaisons; second, the accuracy of answers to 'yes-no' questions cannot be guaranteed when plenty of pipes, ducts and pieces of equipment are crowded together. This method could only be practically applicable if the diagram and Q\&A were automatically generated which brings us back to square one.

\section{A tree structure}

In order to obtain detailed assembly plans, Ben-Arieh and Kramer (Ben-Arieh and Kramer, 1994) presented a methodology and algorithms to consistently generate all feasible assembly sequences considering the various combinations of subassembly operations. Since the complete sequence is represented by a tree structure, from the tree root node to a leaf node, it is called 'a tree structure' by the author. This approach is the 'assembly' or 'forward' approach contrary to the 'disassembly' or 'backward' approach.

They use three types of constraints to limit the number of feasible sequences. These are contact constraints, geometric precedence constraints and technological constraints.

- Contact constraints- Ben-Arieh and Kramer specify which parts are connected to other parts in terms of an assembly operation. For example, an assembly consists of parts $\mathrm{A}, \mathrm{B}, \mathrm{C}$ and $\mathrm{D}$, see figure 4.8. If part $\mathrm{C}$ is connected only to part D, introducing the parts in the order of A, C, B, D will yield an infeasible 


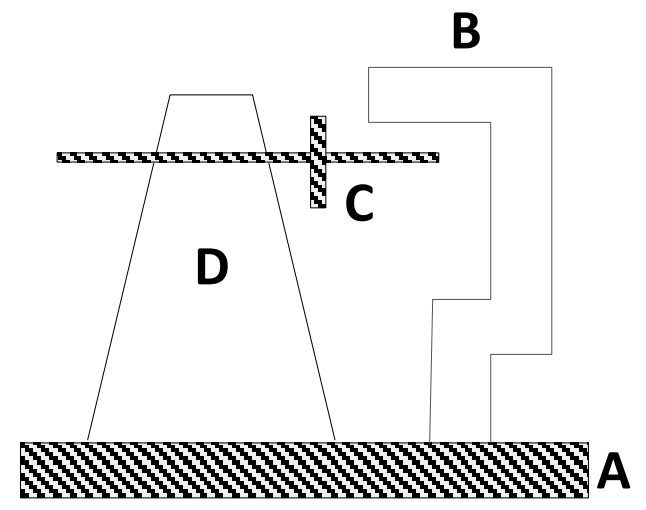

Figure 4.8: Example of an assembly

sequence of assembly operation. In this case, part $\mathrm{C}$ is introduced after part $\mathrm{A}$ and cannot be assembled because it is not in contact with part A.

- Geometric precedence constraints- They represent the fact that some components have to be assembled before the others, otherwise, they will interfere with a later assembly operation. In the example, it is not possible to assemble $\mathrm{C}$ after A, B and D are assembled because there is insufficient space to insert $\mathrm{C}$ into D.

- Technological constraint- It is a precedence relationship that recommends a particular sequence over another based on technological advantage. It is applicable when multiple mating occurs. For example, if a plate has to be welded at one end and screwed to another plate at the other end, then the technological constraint may recommend only one sequence: the plate is screwed first and then welded as the reverse sequence may prevent a successful assembly. In our example it is preferred to start with the base plate A because that lends stability for the subsequent operations.

The input of this algorithm is: list of components, a list of contacts of each component and a list of the precedence constraints. The output is the list of all feasible sequences, represented as a set of trees. Here, the generation of assembly sequences of part A, B, C and D is used to illustrate how this algorithm works.

The algorithm starts by creating four trees with the root nodes A, B, C, and D. Each such node is associated with its contact list. Each such node is expanded by creating a new node for each part on its input list. For example, node A in figure 4.9 is expanded by adding two new nodes $\mathrm{B}$ and $\mathrm{D}$, thus representing the partial 
sequence $\mathrm{A} \Rightarrow \mathrm{B}$ and $\mathrm{A} \Rightarrow \mathrm{D}$. For each node, a new input list is created by merging its contact list with its predecessor's. In the example, node $\mathrm{B}$ has the contact list [A], and its predecessor (node A) has the input list [B D]. The combined list is [A B D]. By eliminating duplicate nodes and nodes already on the path, the new input list is reduced to [D]. In the next iteration, node $\mathrm{B}$ is expanded by adding node $\mathrm{D}$ to its path, and finally node $C$ is added to node $D$, representing the sequence $A \Rightarrow B \Rightarrow D \Rightarrow C$. The other sequences resulting from root node $A$ are $A \Rightarrow D \Rightarrow C \Rightarrow B$ and $A \Rightarrow D \Rightarrow B \Rightarrow C$. In the same principle, the other three trees respectively with the root $\mathrm{B}, \mathrm{C}$ and $\mathrm{D}$ are also derived as shown in figure 4.9. Altogether 8 possible sequences are generated from four trees, which are:

$$
\begin{aligned}
& A \Rightarrow B \Rightarrow D \Rightarrow C ; A \Rightarrow D \Rightarrow C \Rightarrow B ; A \Rightarrow D \Rightarrow B \Rightarrow C ; \\
& B \Rightarrow A \Rightarrow D \Rightarrow C ; \\
& C \Rightarrow D \Rightarrow A \Rightarrow B ; \\
& D \Rightarrow C \Rightarrow A \Rightarrow B ; D \Rightarrow A \Rightarrow B \Rightarrow C ; D \Rightarrow A \Rightarrow C \Rightarrow B .
\end{aligned}
$$

While traversing the tree, the generated sequences are also checked against geometrical precedence constraints and infeasible sequences are eliminated. Thus the tree is pruned and only feasible initial sequences are generated, which are:

$$
\begin{aligned}
& A \Rightarrow D \Rightarrow C \Rightarrow B ; \\
& C \Rightarrow D \Rightarrow A \Rightarrow B ; \\
& D \Rightarrow C \Rightarrow A \Rightarrow B ; D \Rightarrow A \Rightarrow C \Rightarrow B .
\end{aligned}
$$

At last, the technological constraint is used to find the optimized sequence based on technological advantage. The sequence $A \Rightarrow D \Rightarrow C \Rightarrow B$ is the best one compared to the other three because it starts with the base plate $\mathrm{A}$, which provides stability for the subsequent operations.

The contact list is the indispensable input of this algorithm. It indicates that the application of this method requires the components to be assembled must have physical contacts because of the mating with others. However, in the outfitting processes as we pointed out in section 4.1, many components are not mated with others. Thus, to generate the contact lists does not help in pruning the tree. To use this method to generate assembly sequences of outfitting components is therefore not very appropriate.

\subsubsection{The disassembly approach}

\section{AND/OR graph}

In the early 1990s, Homen de Mello and Sanderson (Homem de Mello and Sanderson, 1991b) generated the assembly sequences for a product using a disassembly or decomposition method based on the assumption that the disassembly sequence is the reverse of a feasible assembly sequence. The basic idea underlying the approach is to enumerate the decompositions of the assembly and to select those decomposi- 
Input:

- List of components: [A, B, C, D]

- Contact List:
A: [B D]
B: $[A]$
C: [D]
D: $[A C]$

- List of the precedence constraints:

$C$ cannot be installed after A, B and D are assembled.
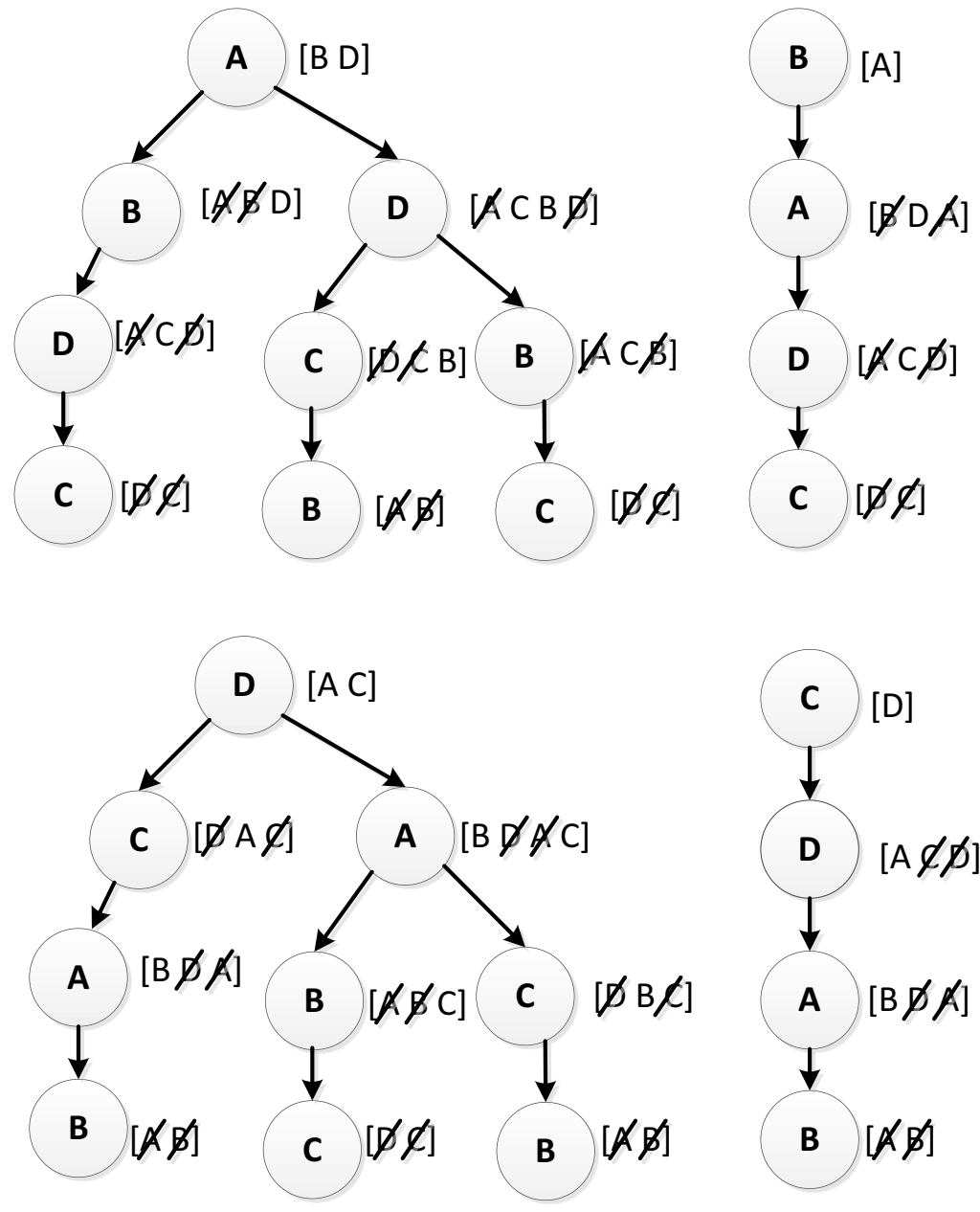

Figure 4.9: Various tree structures of the assembly with four components 


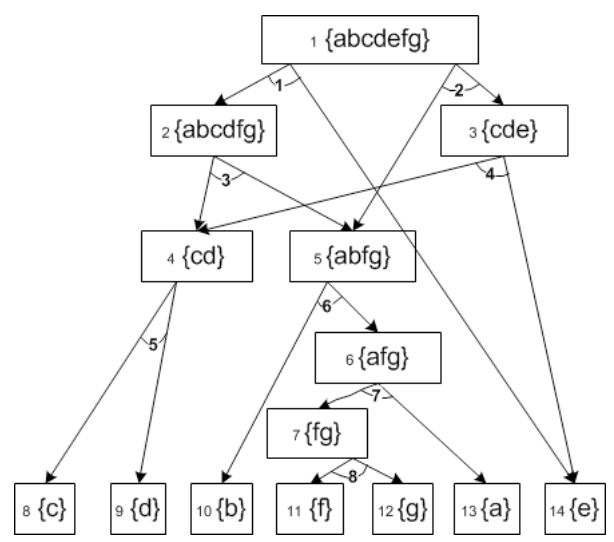

Figure 4.10: The AND/OR graph of the assembly with 7 components

tions that are feasible. Here, Homen de Mello and Sanderson (Homem de Mello and Sanderson, 1990), (Homem de Mello and Sanderson, 1991a) proposed an AND/OR graph representation for all the possible decompositions.

As an illustration, we use the AND/OR graph shown in figure 4.10 of the outfitting assembly consisting of the 7 components in figure 4.5 as an example to explain the AND/OR graph, which is represented by $\mathrm{G}=(\mathrm{W}, \mathrm{D})$.

$\mathrm{W}-$ Each node in $\mathrm{W}$ can be a product, part, or subassembly. In figure 4.10, node $1\{\mathrm{abcdefg}\}$ is a product, node $5\{\mathrm{abfg}\}$ a subassembly and node $14\{\mathrm{e}\}$ a part.

D- Hyperarcs D represent the set of feasible disassembly tasks. In figure 4.10, hyperarc 1 is a task to disassemble node 1 \{abcdefg\} into node 2 \{abcdfg\} and node $14\{\mathrm{e}\}$.

A node $\mathrm{i}$ representing a product or subassembly can have $\mathrm{k}(\mathrm{k} \geq 1)$ disassembly methods, forming an OR-relation. In figure 4.10, since hyperarc 1 and 2 are two different methods to disassemble node 1 abcdefg\}, the relation between them is an OR relation; if a method disassembles $i$ into $u(u \geq 2)$ nodes, $u$ arcs link node $i$ to those $\mathrm{u}$ nodes, forming an AND relation, here node 1 abcdefg\} is disassembled into node $3\{\mathrm{cde}\}$ and node $5\{\mathrm{abfg}\}$, thus they form an AND relation.

In this example, pipe $b$ and cable tray e are transverse to the main axis of the ship (from stern to bow) and the others are longitudinal. Two disassembly methods can be generated because of the two transitions of main axis direction. Thus the disassembly order of the outfitting shown in the graph is $e \Rightarrow(c, d) \Rightarrow b \Rightarrow(f, g) \Rightarrow a$.

Unlike the assembly in the manufacturing industry in which the construction of the AND/OR graph is based on the ability to find all stable subassemblies and depends much more on the physical contacts between parts of a product, intersection (called 'collision') of outfitting components is not frequent. This implies even if an 
54 Automatic generation of assembly sequence for the planning of outfitting processes in shipbuilding

outfitting component or a subassembly is removed from the remaining assembly of outfitting components in steel structures, the whole system still remains stable. Thus it is not feasible to disassemble outfitting into subassemblies according to the criterion if the subassembly is stable or not. Meanwhile, the resultant AND/OR graph is large and the number of nodes grows exponentially as the number of components in the assembly increases. To determine and store all (feasible) assembly sequences consumes large amounts of computer processing time and memory. Therefore, the application of this method for our purpose is debatable.

\section{Binary matrix}

Another disassembly method was put forward by Dini and Santochi(1992) (Santochi and Dini, 1992). They developed a mathematical model to detect subassemblies and select the assembly sequences for a product. In order to express the accurate knowledge of the position and connection relationships among the components of a product, this model defines 3 matrices: the interference matrix, the contact matrix and the connection matrix. Their explanations are as follows:

- A=Interference matrix

It detects all the collisions during the translation along the direction $+/-\mathrm{k}$ until the element is completely separated from any part of the product. The direction $\mathrm{k}$ can be $\mathrm{XYZ}$ of the Cartesian coordinate system of the CAD space. Thus, the interference matrix $\mathrm{A}_{k}$ of a product formed by $\mathrm{n}$ elements $\mathrm{e}_{1}, \mathrm{e}_{2}, \ldots, \mathrm{e}_{n}$ is defined as

$$
A_{k}=\begin{gathered}
e_{1} \\
e_{2} \\
e_{2} \\
\ldots \\
e_{i} \\
\ldots \\
e_{j} \\
\ldots \\
e_{n}
\end{gathered}\left[\begin{array}{cccccccc}
a_{1,1} & a_{1,2} & \ldots & a_{1, i} & \ldots & a_{1, j} & \ldots & a_{1, n} \\
a_{2,1} & a_{2,2} & \ldots & a_{2, i} & \ldots & a_{2, j} & \ldots & a_{2, n} \\
\ldots & \ldots & \ldots & \ldots & \ldots & \ldots & \ldots & \ldots \\
a_{i, 1} & a_{i, 2} & \ldots & a_{i, i} & \ldots & a_{i, j} & \ldots & a_{i, n} \\
\ldots & \ldots & \ldots & \ldots & \ldots & \ldots & \ldots & \ldots \\
a_{j, 1} & a_{j, 2} & \ldots & a_{j, i} & \ldots & a_{j, j} & \ldots & a_{j, n} \\
\ldots & \ldots & \ldots & \ldots & \ldots & \ldots & \ldots & \ldots \\
a_{n, 1} & a_{n, 2} & \ldots & a_{n, i} & \ldots & a_{n, j} & \ldots & a_{n, n}
\end{array}\right]
$$

Here $\mathrm{a}_{i, j}=1$ if the element $\mathrm{e}_{i}$ collides with the element $\mathrm{e}_{j}$ during the translation of $\mathrm{e}_{i}$ (by whatever distance) along the direction $+\mathrm{k}$, otherwise $\mathrm{a}_{i, j}=0$. By convention $\mathrm{a}_{i, i}$ is always equal to zero since it is not possible for the component to interfere with itself no matter along which direction it is translated. The transpose matrix $\mathrm{A}_{+k}^{T}$ represents the interference along the opposite direction $-\mathrm{k}$.

- $\mathrm{B}=$ Contact matrix 
The contact matrix $\mathrm{B}_{k}$ of a product formed by $\mathrm{n}$ elements $\mathrm{e}_{1}, \mathrm{e}_{2}, \ldots, \mathrm{e}_{n}$, is the square matrix of order $\mathrm{n}$ where $\mathrm{b}_{i, j}=1$ if the element $\mathrm{e}_{i}$ is in contact with the element $\mathrm{e}_{j}$ along the direction $+\mathrm{k}$, otherwise $\mathrm{b}_{i, j}=0$. As a convention $\mathrm{b}_{i, i}$ is always equal to zero.

Both the interference matrix and the contact matrix are derived from the CAD simulation of each of the component translations; the former is obtained detecting all the collisions until the component is completely separated from the product, the latter detecting only the immediate intersection.

- $\mathrm{C}=$ Connection matrix

It describes the kind of connection existing between elements. The kinds of connection can be 'threaded connection', 'drive fit', 'elastic ring', etc. and are represented by a numerical code (such as $1,2,3$ ) by Dini and Santochi. The connection matrix $\mathrm{C}_{k}$ of a product formed by $\mathrm{n}$ elements $\mathrm{e}_{1}, \mathrm{e}_{2}, \ldots, \mathrm{e}_{n}$, is the square matrix of order $\mathrm{n}$ where each $\mathrm{c}_{i, j}$ assumes a numerical code that represents the kind of connection existing between the element $\mathrm{e}_{i}$ and $\mathrm{e}_{j}$ along the direction $\mathrm{k}$. For example, $\mathrm{c}_{i, j}=1$ means the element $\mathrm{e}_{i}$ is joined to the element $\mathrm{e}_{j}$ through a threaded connection along the direction $\mathrm{k}$ and $\mathrm{e}_{i}$ represents the element to be disassembled. If conversely $\mathrm{e}_{j}$ is to be disassembled, $\mathrm{c}_{i, j}=-1$.

The process for the detection of the subassemblies takes into account the similar position of components. If the following condition is satisfied: $\mathrm{a}_{i, m}=\mathrm{a}_{i, j}(1 \leq$ $\mathrm{i} \leq \mathrm{n}$ and $\mathrm{m} \neq \mathrm{j}$ ), it indicates component $\mathrm{m}$ and $\mathrm{j}$ have the same relationship with the remaining components and can be grouped as a subassembly. Therefore, from the interference and connection matrices, the system detects the elements or subassemblies to be disassembled and the direction of the translations, obtaining all the possible ways of disassembly; when an element or a subassembly is separated from a set, the matrices are updated to the new configuration, setting to zero the elements of the corresponding row and column or indeed deleting the row and column. The detection is repeated until all subassemblies are disassembled.

Based on the fact that no element of a product is in the 'air', this method should be modified when applied to the outfitting assembly where no or very few components in space are physically in contact with one another as we discussed before. Thus matrix $\mathrm{B}$ and $\mathrm{C}$ will be less relevant in the outfitting case.

Here, we take the assembly of the seven components in figure 4.5 as an example to explain the application of the interference matrix to the outfitting process.

According to the definition, when translating the components either along the $+\mathrm{z}$ direction (from the bottom to the top of a ship) or along the $-\mathrm{z}$ direction, the interference matrices $\mathrm{A}_{+z}$ and $\mathrm{A}_{-z}$ of this example can be obtained as: 
56 Automatic generation of assembly sequence for the planning of outfitting processes in shipbuilding

$$
\begin{aligned}
& A_{+z}=\begin{array}{ccccccc}
a & b & c & d & e & f & g \\
a \\
b \\
c \\
d \\
e \\
f \\
g
\end{array} \quad\left[\begin{array}{ccccccc}
0 \\
1 & 0 & 0 & 0 & 0 & 0 & 0 \\
0 & 1 & 0 & 0 & 0 & 0 & 0 \\
0 & 1 & 0 & 0 & 0 & 0 & 0 \\
1 & 0 & 1 & 1 & 0 & 1 & 1 \\
0 & 0 & 0 & 0 & 0 & 0 & 0 \\
0 & 0 & 0 & 0 & 0 & 0 & 0
\end{array}\right] \\
& \left.\left.A_{+z}^{T}=A_{-z}=\begin{array}{lllllll}
a & b & c & d & e & f & g \\
a \\
b \\
c \\
d \\
e \\
f \\
g
\end{array}\right] \begin{array}{lllllll}
0 & 1 & 0 & 0 & 0 & 0 & 0 \\
0 & 0 & 1 & 1 & 0 & 0 & 0 \\
0 & 0 & 0 & 0 & 1 & 0 & 0 \\
0 & 0 & 0 & 0 & 1 & 0 & 0 \\
0 & 0 & 0 & 0 & 0 & 0 & 0 \\
0 & 1 & 0 & 0 & 0 & 0 & 0 \\
0 & 1 & 0 & 0 & 0 & 0 & 0
\end{array}\right]
\end{aligned}
$$

In the matrix $\mathrm{A}_{+z}, \mathrm{a}_{2,1}=1, \mathrm{a}_{2,6}=1$ and $\mathrm{a}_{2,7}=1$ imply that the translation of pipe $\mathrm{b}$ along the $+\mathrm{z}$ direction will be hindered by pipe $\mathrm{a}$, $\mathrm{f}$ and $\mathrm{g}$. In the matrix $\mathrm{A}_{-z}, \mathrm{a}_{2,3}=1$ and $a_{2,4}=1$ indicate that the translation of pipe $b$ along the $-\mathrm{z}$ direction is blocked by pipe $c$ and d. In this way, the components that interfere with pipe $b$, either above or below, can be detected when the translations along the $\mathrm{z}$ direction are carried out.

\subsubsection{Discussion}

These assembly methods (liaison diagram, a tree structure, AND/OR graph and binary matrix) comprise the major methods of assembly sequence generation reported in literature. When considering their application to the outfitting in the shipbuilding industry, one major difference between the assembly of a product and that of the outfitting assembly is the physical contact as we pointed out in section 4.1. All these methods take the physical contact as a major constraint. Through the analysis of how each component contacts with others, they try to generate feasible assembly sequences. However, there exist relatively few physical contacts between outfitting components dominating their mounting order. The possibilities of their applications in our research are discussed as follows:

- Liaison diagram: the liaison diagram is the first creative step in generating the family assembly sequences. It requires the practitioner to be able to anticipate some possible assembly sequences. Thus, this step much depends on human labor. The second step, to answer Yes-No questions, also requires the practitioner to give the answer. In the outfitting, the number of components in a section may easily run into the hundreds. When the number of components 
increases, it is not easy for the practitioner to first generate a largely reasonable liaison diagram and then answer Yes-No questions properly. This conflicts with our research purpose-to automate the assembly sequence generation process. Thus it is not practical to apply this method in our research.

- A tree structure: the application of this method requires that the components to be assembled have physical contacts because of the mating with others. However, in the outfitting processes, many components are not mated with others. Thus, to generate the contact lists is fairly irrelevant. To use this method to generate assembly sequences of outfitting components is problematic.

- AND/OR graph: it is not practically relevant for this method to disassemble an outfitting assembly into subassemblies according to the criterion if the subassembly is stable or not. Meanwhile, the resultant AND/OR graph is large and the number of nodes grows exponentially as the number of components in the assembly increases. To determine and store all (feasible) assembly sequences is expected to consume large amounts of computer processing time and memory. The application of this method need to consider issues of computer load and operator work involved in using it. Therefore, its application is thought debatable.

- Binary matrix: the contact matrix $\mathrm{B}$ and the connection matrix $\mathrm{C}$ will be applicable to a much smaller extent in the outfitting case. The interference matrix is selected to express the physical relationship between the outfitting components in a ship's section for two reasons.

The first is that the function of this matrix is to detect whether there exists a collision/interference between two components or not. It makes sure no other components are in the way during the assembly. It is thus immediately related to the feasibility of the assembly. To be able to generate a collision-free sequence is the basic requirement of the research. The second reason is the availability of 3-D coordinates of each component which makes it possible to formulate the matrix. Besides, a test case with 400 components shows that the computer time for the matrix is less than 30 seconds which is also quite good for practical applicability of the method.

\subsection{Summary}

Assembly work of outfitting processes in shipbuilding and that in the manufacturing industry bear a high resemblance to each other. The same characteristics have to be considered such as geometrical attributes of each component, dependencies between components and the necessity to evaluate various assembly operations. In addition, 
58 Automatic generation of assembly sequence for the planning of outfitting processes in shipbuilding

the planners have to answer the same questions: find a practicable sequence of assembly operations while maximizing the production profitability and resource utilization.

Based on the previous assembly sequence researches done in other manufacturing industries, this chapter illustrates the widely used assembly methods with the potential to be used in outfitting processes. Through the comparison of the definition of components in the manufacturing industry and that in outfitting, and the assembly attributes, it is expected that the technology of 'assembly sequence planning' can be used in outfitting processes in shipbuilding. Besides, four assembly methods (liaison diagram, a tree structure, AND/OR graph and binary matrix) are explained in detail. The possibilities of their applications in our research were discussed in section 4.2. In conclusion, the interference matrix, part of the binary matrix method, is provisionally selected to detect the relationships between outfitting components. 


\section{Chapter 5}

\section{An Overview of the Structure of the Assembly Sequence Generation System}

The previous chapters focused on the problem identification in outfitting processes in shipbuilding and emphasized the need for an assembly sequence planning system. Besides, the fundamental theory of this system has also been laid and described in chapter 4. It adopted the disassembly or decomposition approach under the assumption that the feasible assembly sequence is the inverse of the disassembly sequence if there is a feasible solution for the disassembly. One of the typical disassembly methods, called 'Binary Matrix' was chosen to detect the physical relationships between components. Based on this foundation, the structure of this system is established in this chapter. The goal of this chapter is to provide a holistic view of the assembly sequence generation system as displayed in figure 5.1, which will be described in detail in the following four chapters.

Chapter 6 has two major functions:

$\begin{gathered}\text { Chapter } 6 \\ \begin{array}{c}\text { Chysical attributes } \\ \text { analysis } \\ \text { What information are we } \\ \text { interested in? }\end{array} \\ \text { Data collection and preparation } \\ \text { Where to get these information? } \\ \text { How to translate these } \\ \text { information into useful input data? }\end{gathered}$

Figure 5.1: The structure of assembly sequence generation system 
60 Automatic generation of assembly sequence for the planning of outfitting processes in shipbuilding

1. To establish a conceptual assembly sequence generation model from observations of outfitting assembly work in shipyards;

It explores:

- What kind of geometry attributes of outfitting components are important in the determination of their mounting orders?

- How to detect the physical relationships between components?

- How do the geometry attributes and physical relationships affect their mounting orders?

2. To interpret the conceptual model mathematically.

Based on the possible assembly methods and representations introduced in chapter 4 , it describes the way how to interpret these attributes mathematically with a purpose of making them programmable. Weighting coefficients, represented by the module 'Weighting coefficients' in figure 5.1, are used to quantify the impact of the components' geometry attributes on the mounting orders. They are calculated using the analytical hierarchy process (AHP) method, applied to questionnaire results.

Once the attributes related to the components' geometry and the ones related to the physical relationship between the components are clear, chapter 7 describes how to obtain this information and how to transform it into a proper input format, ready to be accessed by the model. For our own laboratory purpose, the detailed engineering CAD model, generated by TRIBON, was chosen to be the original data source. A data preparation model is necessary to reorganize the data in the format that it can be read by the sequence generation model. Apart from this, it is also able to extract the necessary information, like diameters of pipes, which is implicit in the original data without being explicitly stated. All equipment information can be found in another database, represented by the module 'Equipment DB' in figure 5.1.

Taking the data from the data preparation module and the equipment database as input, chapter 8 mainly focuses on how the sequence generation model works. Here, the 'basket done' method is introduced. It enables the model to generate the assembly activities in parallel. Three outputs from the model are as follows:

- Visualization of the assembly sequence by Gantt Chart, which shows throughput time of each task and start-finish relationships among installation activities.

- Recording the assembly sequence by means of a Excel file, which allows other planning tools, like Mars Planning, Microsoft Project, Primavera, etc., to access it.

- Visualizing components in a 3D view, which provides planners with the animation of the sequence. 


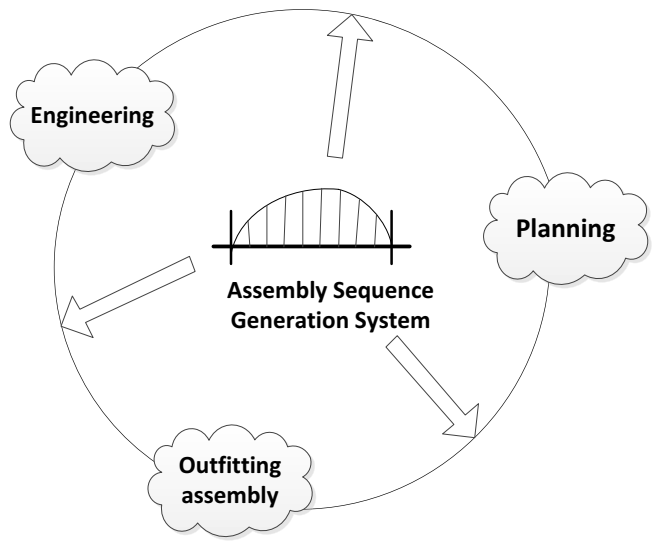

Figure 5.2: A supporting tool for outfitting planning and outfitting assembly

Chapter 9 presents the model application and evaluation. It is used to describe the verification of the mounting time of assembling each type of components and the application of the method for four test cases. The differences between the duration of the model and that of the pre-outfitting plan are analyzed. By discussing the results with expert outfitting employees of shipyards, the limitation and the further improvements of the model are also discussed here.

Since the assembly sequence generation system mainly captures the assembly knowledge in terms of technical aspects and answers the questions 'what has to be accomplished in outfitting processes?' and 'In what order will it be accomplished?', it is not intended to be another planning tool of which the function is to commit resources to a variety of possible tasks. So far, this system is not an assembly instruction tool either, because it has not been incorporated into an e-viewer of the engineering model, which allows visualization of real scenarios. Nevertheless, the assembly sequence generation system can be regarded as a supporting tool for outfitting planning and outfitting assembly. As shown in figure 5.2, this system not only helps planners to translate the engineering drawings into a more realistic outfitting plan but also deepens their understanding of how outfitting assembly work is proceeding; moreover, it provides production staff with outfitting assembly knowledge and helps them translate engineering drawings into final products. As such it bridge the current gaps between engineering and planning; planning and outfitting assembly; and engineering and outfitting assembly.

The entire assembly sequence generation system should be able to: 
62 Automatic generation of assembly sequence for the planning of outfitting processes in shipbuilding

- Interface with external systems, like CAD models and planning tools, such as Mars Planning, Microsoft Project, Primavera, etc.

- Support the planner by proposing valid relations for assembling outfitting components. When the planner makes a pre-outfitting plan, the proposed relationships for each task together with its duration time helps him to schedule human and facility resources more realistically at a higher level of detail.

- Capture outfitting assembly knowledge in such a way that it enables generation of a realistic assembly sequence comparable to a real-world outfitting process. 


\section{Chapter 6}

\section{Physical Attributes Analysis}

In the pre-outfitting stage when sections are open, workers mount components according to a certain (but undefined) sequence. Figure 6.1 is an example. The left part of the figure shows the early stage, during which a few pipes were already mounted; in the middle (taken from the opposite direction), the picture shows the situation one day later; on the right hand side of the picture more pipes were added to the section three days later. Why were these pipe spools installed in this sequence?

According to Arieh (Ben-Arieh, 1994), when considering a feasible assembly sequence in terms of technical aspects, two types of attributes of the components involved are of significance: attributes related to the components' geometry, and ones related to the physical relationship between the components. Both not only decide the feasibility of assembly operations, but also influence the degree of difficulty to accomplish them. Thus, to answer the above question, another three questions need to be addressed:

- What kind of geometry attributes of outfitting components are important in the determination of their mounting orders?

- How to detect the physical relationships between components?

- How do the geometry attributes and physical relationships affect their mounting orders?

These questions will be answered in this chapter through the analysis of physical attributes of outfitting components. Each section is devoted to answering one question and the summary is made in section four.

\subsection{Physical attributes of components}

According to chapter 2.2, four main categories of assembly work in outfitting processes are included in the research: pipes, electrical cable trays, HVAC and equip- 
64 Automatic generation of assembly sequence for the planning of outfitting processes in shipbuilding

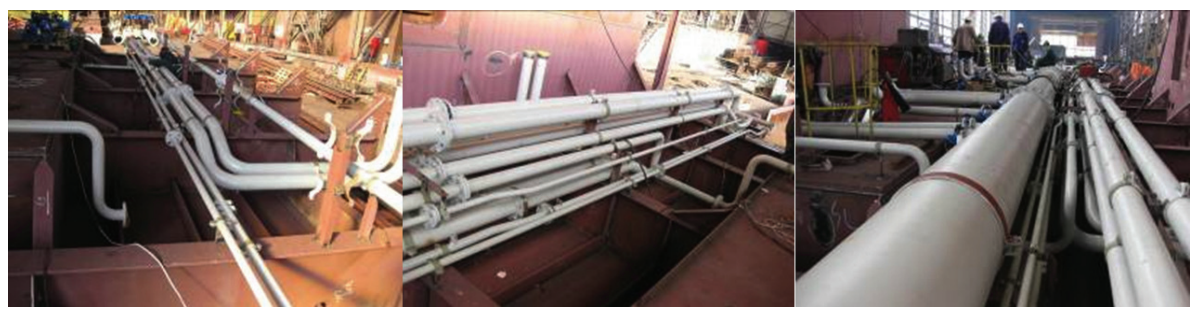

Figure 6.1: Pipes are mounted in a certain sequence

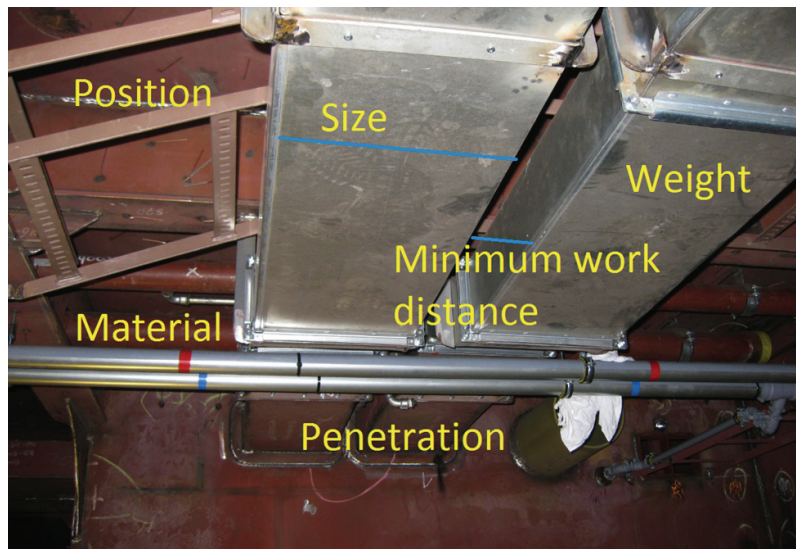

Figure 6.2: Attributes of outfitting components

ment installation. When investigating what physical attributes of outfitting components are important in the determination of their mounting orders, they should preferably be general enough so that they can be applied for all these four types of components but also representative in order that they are sufficient to determine the assembly sequence. Furthermore, the attributes should be sufficiently simple so that the information can be expected to be available early enough to support the planning process.

After the field observation and thorough consultation with workers, the following attributes of components have been chosen and are shown in figure 6.2: position, material, weight, size, penetration and minimum work distance. How they influence the components' assembly sequences is explained in detail in the rest of this section in which we also endeavor to quantify such influence.

\subsubsection{Position}

When considering the feasible assembly operations, it would be practical to first install components closest to the steel structure boundaries, like walls, floors or ceilings. Then the components located further from boundaries can be gradually added 


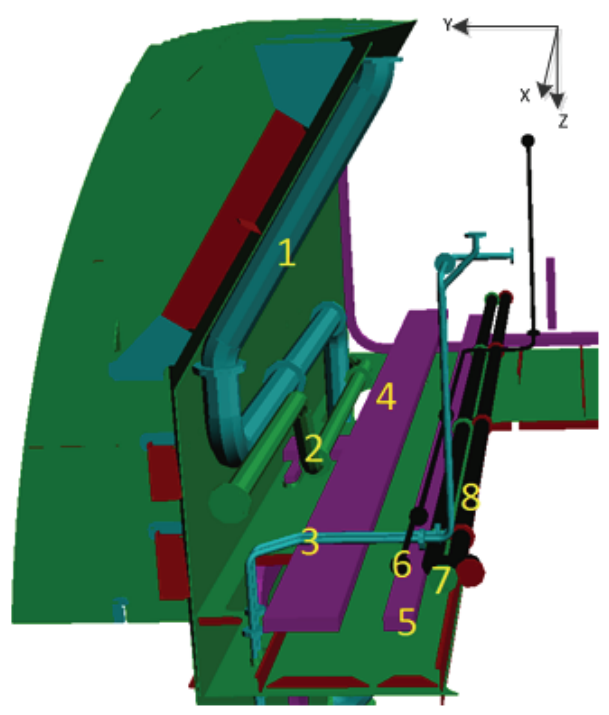

Figure 6.3: 8 components in a steel section

in. This guarantees in most cases that no other outfitting components are in the way when installing (locating and positioning) a certain component. Thus, a collisionfree path is created. Take the installation of 8 components in a steel section shown in figure 6.3 as an example. The front view of this steel section can be found in figure 6.4 .

In this example, pipe 1 is closer to the portside shell steel structure than pipe 2. Thus, the installation of pipe 1 is followed by that of pipe 2 . Among pipe 3 , cable tray 4,5 and pipe 6 , cable tray 4 and 5 are closest to the deck and thus, probably assembled first. The installation of pipe 3 precedes that of pipe 6 which is above pipe 3 .

From this aspect alone, the position of the component within a steel section, when taking it to extremes, completely decides its installation order in the assembly process. In the research, 'position' specifically links to the detection of interferences between outfitting components. This will be explained in chapter 6.2.

As a convention in the 3D model, the position is expressed in terms of three dimensional coordinates and generated during the engineering stage. For example in figure 6.5, the position of a piece of pipe consisting of 4 spools is defined by the coordinates of end points and bend points along its axis. A piece of equipment (a central heating boiler) is expressed by the coordinates of 8 corner points of its boundary box, see chapter 7.1.3. In this way, an outfitting component is 'located' in a vessel. Here, the Cartesian coordinate system of a vessel shown in figure 6.6 is defined as follows: positive $\mathrm{X}$ is from the rudder post forward, positive $\mathrm{Y}$ is from the mid-ship portside and positive $\mathrm{Z}$ is from the keel upward. The global origin $\mathrm{O}$ is 


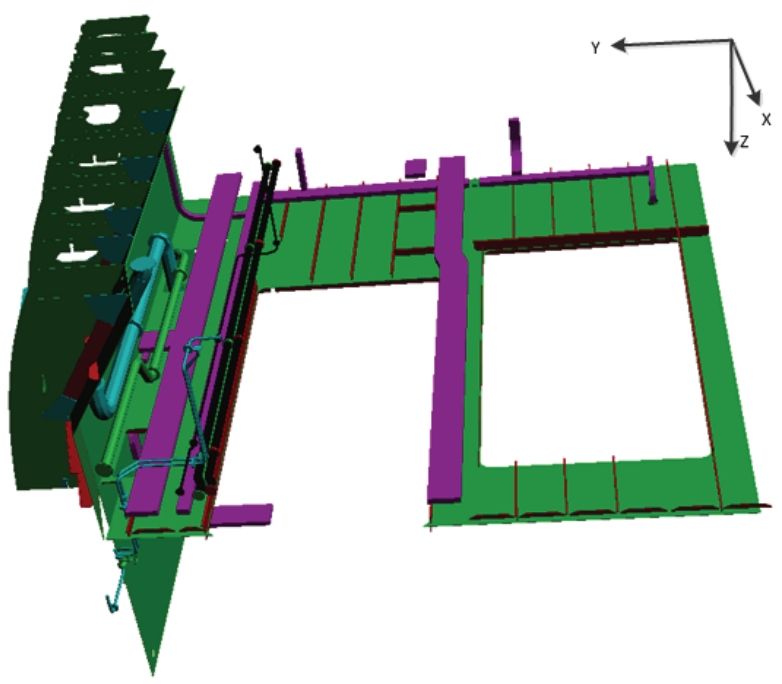

Figure 6.4: The front view of the steel section

located at the zero frame of a vessel.

\subsubsection{Material}

The possibility for an installed component to be damaged by later assembly operations is directly related to the material this component is made of. For instance, the welding of brackets for a steel pipe may cause the damage to a sheet metal duct adjacent to this pipe that has already been perfectly positioned and supported. Therefore, rework occurs and cost increases.

Apart from steel and sheet metal, more and more components are made of polyvinyl chloride (PVC) and light metal alloys. Especially plastic pipes are widely used for their light weight, chemical resistance, anti-corrosive properties, and ease of making connections. On the other hand, their installation during the production processes requires more attention due to the fragility. It implies that the material aspect also plays a role in the determination of the assembly sequence and should be given attention.

How to quantify this aspect? By definition, the more fragile the material is, the greater is the possibility that it may be damaged. Thus, a rule is set that the more fragile materials need to be disassembled earlier (i.e. assembled later). In the research, we choose five most common types of material to depict the material attribute of each outfitting component. These are: steel, spiral metal, rectangular metal, plastic and copper. The method to quantify the material's influence on the assembly order of components is developed as:

Step1: The components are categorized based on their material: steel, spiral metal, rectangular metal, plastic, copper, etc. 


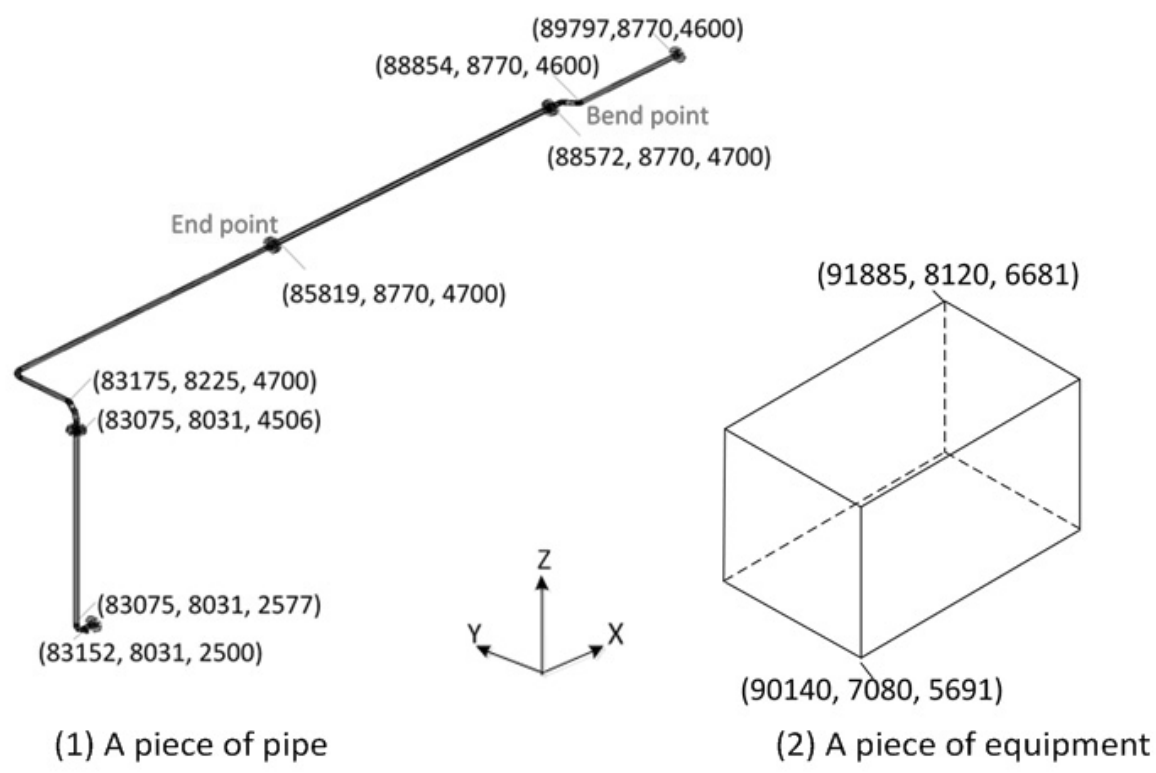

Figure 6.5: A piece of pipe and equipment with their coordinates
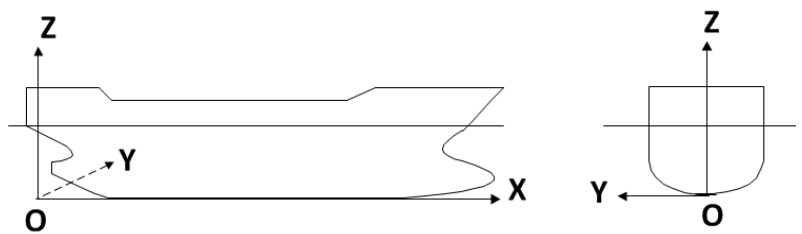

Figure 6.6: Cartesian coordinate system of a ship 
Step2: A value $\alpha$ is assigned to each category according to its priority to be assembled. For instance, if the category of steel should precede all other categories, it should be given the highest $\alpha$ value. If the category of copper should follow all other categories, it should be given the lowest $\alpha$ value.

Step3: Rank the components according to their corresponding $\alpha$ in descending order. It makes sure that components with stronger material enjoy higher priority to be assembled.

For equipment, the fragility is not suitably defined by its overall material. Rather, it may be more related to internal connections, i.e. what kind of components a piece of equipment is composed of and how these are connected. Most pieces of equipment in a ship are made of steel material. If they are partly composed of fragile components, like sensors, electrical wires, or other measuring apparatuses, their installation could be carried out at the later outfitting stage to reduce the chances of damaging these fragile components. Alternatively, they can be protected e.g. by a wooden casing, after placing them. Although in our research all equipment are treated as steel (and thus assumed to be very sturdy, with or without protection), their fragility needs to be considered in more detail in future improvements to this method.

\subsubsection{Weight}

Most outfitting components weigh more than $25 \mathrm{~kg}$. According to health and safety regulations in construction (Hughes and Ferret, 2008), manual handling of pipes or equipment can lead to musculoskeletal health problems and components weighting more than $25 \mathrm{~kg}$ should not be carried by a single worker. In most cases logistic resources, such as cranes, trucks and forklifts, to transport certain components from storehouse to the appointed assembly area are needed. Thus, weight is associated with ease or difficulty of handling.

Since during the early assembly stage the space is more easily accessible for handling/lifting equipment and the materials and equipment are closer at hand, the heavier outfitting components are, the earlier they are preferably assembled. Otherwise, the obstruction from structural components would hinder the accessibility to hoist and lower the heavier components or equipment into the right position, which adds to the indirect time required to perform an assembly or erection task. Meanwhile, if the component of which the weight is beyond the capacity of a chain block, is close to the ceiling of a compartment, it must be assembled during the section building stage, since at that moment it is possible to use the cranes to move it into the up-side down section. Otherwise, there is no means to lift it up to the ceiling. Therefore, weight also plays a role in the determination of components' assembly orders.

The weight information of each component can be obtained from the detailed 3D model. Indeed, it is customary to exclude smaller or light-weight components from the CAD-models, for example, pipes less than $12.50 \mathrm{~mm}$ in diameter. They 
are however often specified in the bill of material (BOM) that has to be acquired by procurement but locations of these smaller components are in that case normally not available. Therefore, they do not allow inclusion in automatic sequence generation in the research.

For components of which the weight is available in 3D model, rank the components according to their weight in descending order. This way makes sure that heavier components enjoy higher priority to be assembled.

\subsubsection{Size}

The word 'size' refers to how big something is. In terms of dimensions, it includes length, width, height, diameter, perimeter, area and volume. In outfitting, the bigger components are, the larger area they will occupy in confined spaces and the larger the openings that are needed to put them in. For example, a piece of duct $700 \mathrm{~mm}$ in width, $1500 \mathrm{~mm}$ in length and $250 \mathrm{~mm}$ in height may be put inside the section in a much easier way when the compartment is still open allowing access from above. This type of attribute is primarily concerned with the spatial accessibility and ease of handling. Thus, the size of components also has the impact on their mounting orders. Which size parameter is decisive heavily depends on the situation. Transportation routes, access openings and compartment dimensions all influence this.

In the research, we take the outside diameter for pipes or width for rectangular ducts and cable trays as an indicator of their sizes. The reasons are that they dominantly decide whether it is easy or not handle the components during the assembly; and that they are explicitly defined in the CAD model as opposed to the parameter 'length'. We will discuss the derivation of the length later in chapter 7.2.

In particular, 'width' means the length of the transverse section that is perpendicular to the flow direction, i.e. the direction in which the air, water or electricity flows. Figure 6.7 shows the width of a duct and that of a piece of a cable tray. For equipment, the size is represented by the largest value among its length, width and height. Through the comparison of the 'size', components with bigger size will be assembled earlier.

\subsubsection{Penetration}

A pipe or a duct spool, which goes through a wall or watertight bulkhead, is called a 'penetrating piece'. During assembly processes, they are regarded as reference pieces and in most situations they are the first ones to be mounted because of their rather precise position in the steel structure. Violating this constraint may bring forth re-assembly or modification of spools, both of which are time-consuming processes. Therefore, whether a component is a 'penetrating piece' to some extent also decides its mounting order. 
70 Automatic generation of assembly sequence for the planning of outfitting processes in shipbuilding
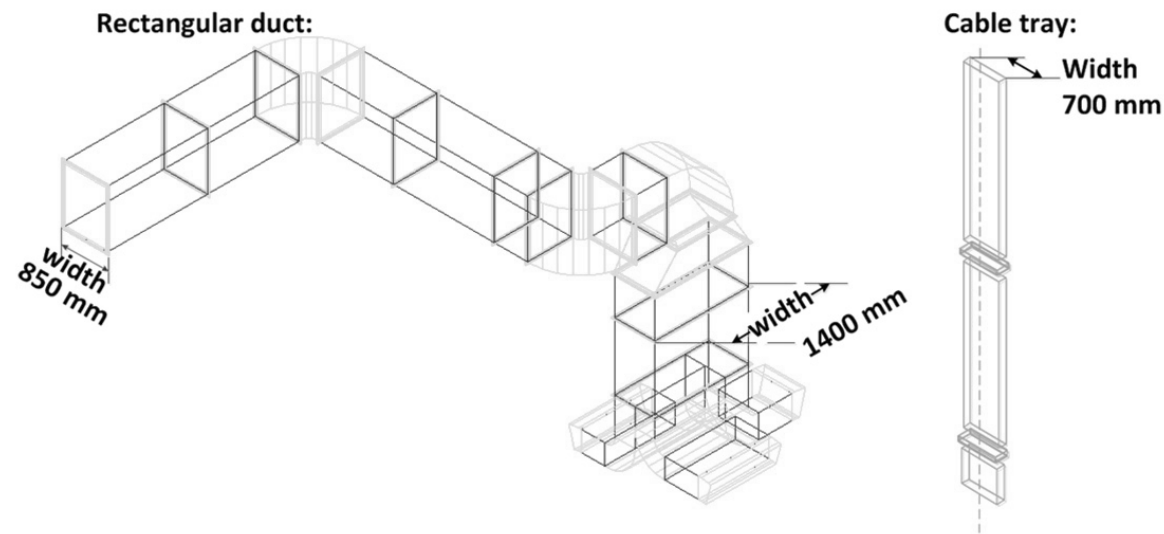

Figure 6.7: Width of rectangular ducts and cable trays

In the 3D model, the penetrating spools often have distinctive names beginning with a letter 'P'. Thus, through their names, the priority to install them earlier is indicated.

\subsubsection{Minimum work distance}

It is recognized that favorable ergonomic conditions on the work site lead to an increased freedom of action for the workers (Bullinger et al., 1997). Sirajuddin (Sirajuddin, 1991) and Thabet and Beliveau (Thabet and Beliveau, 1994) also propose that construction workspace is a combination of different resources, including equipment and tools. In the outfitting processes, workers need sufficient space to position themselves with all kinds of tools, like a welding torch, grinding machine and screw spanner. On the work site, the installation of several spools at the same time takes place frequently when the work space is sufficiently big. This means that the minimum work distance that allows two spools to be mounted simultaneously also influences the assembly sequence.

The definition of the 'minimum work distance' can be found on the left hand of the figure 6.8 when two workers assemble two spools in the back-to back position. In such a scenario, the distance between two spools should be at least equal to or bigger than the minimum work distance if they are to be assembled at the same time. In general, two workers work together as a team to assemble a spool. In such a scenario, the second worker in this team is either busy with measuring the position of spool supports or busy with making supports in the warehouse.

However, in the scenario when workers work face to face as displayed on the right hand of the figure 6.8, there is no requirement for the minimum work distance. In the research, where we disregard the steel structure information, it is not possible to decide which scenario is applicable when two workers assemble two spools at the 

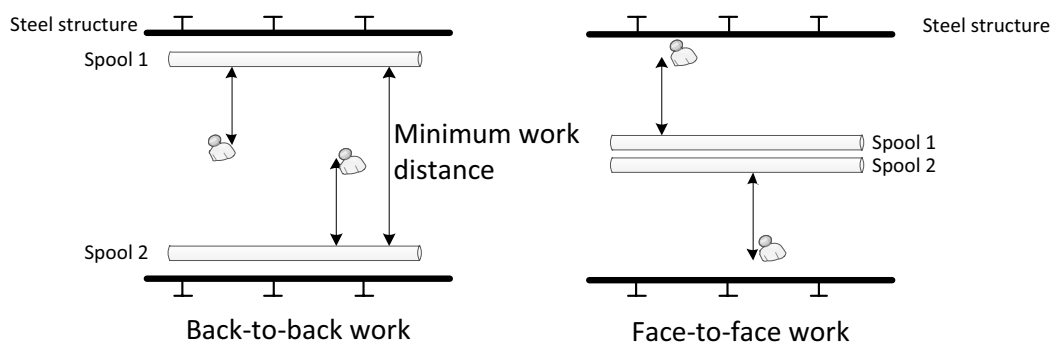

Figure 6.8: Two extreme scenarios of simultaneously assembling two spools

same time. Thus, we assume the minimum work distance is an indispensable prerequisite to decide if two spools can be assembled simultaneously.

There is scarcely literature providing a definite value of minimum work distance when looking at the environment of assembly work in the shipbuilding industry, where the surroundings are full of steel structures. The field observation showed that workers constantly are either in a kneeling, bending or squatting position when they do the measuring with rulers, grinding with grinders or welding with welding torches. The photos in figure 6.9 are just a few examples among many. Thus, the maximum horizontal reaching distance by workers becomes a crucial aspect for the minimum work distance.

Field observations and interviews with pipe fitters showed that the minimum work distance between two workers, who install two different spools separately at the same time, falls into the range of 2-3 m. This fact corresponds with the graphics and tables used to describe the maximum horizontal reaching, provided by Steenbekkers. Figure 6.10, reproduced from (Steenbekkers and van Beijsterveld, 1998), illustrates that the maximum average horizontal reaching distance of a person is $120 \mathrm{~cm}$ and the minimum is $82 \mathrm{~cm}$. Thus, for two persons who work opposite to each other, this distance is between $164 \mathrm{~cm}$ and $240 \mathrm{~cm}$. Since the measurements were taken in the Netherlands where the tallest people in the world live, with the average height of $1.81 \mathrm{~m}$ for males (Frenken, 2008), it should be mentioned that this range only applies to Northern Europeans. For the purpose of this research, the minimum work distance is set as $2.5 \mathrm{~m}$. Figure 6.11 shows the number of $2.5 \mathrm{~m}$ consist of 2 times of $1.2 \mathrm{~m}$ and $0.1 \mathrm{~m}$ between the backs of two workers.

In summary, the above physical attributes of components all play a role in the determination of assembly sequences. The relevant information can be obtained through the detailed engineering 3D model, which are as follows: 
72 Automatic generation of assembly sequence for the planning of outfitting processes in shipbuilding

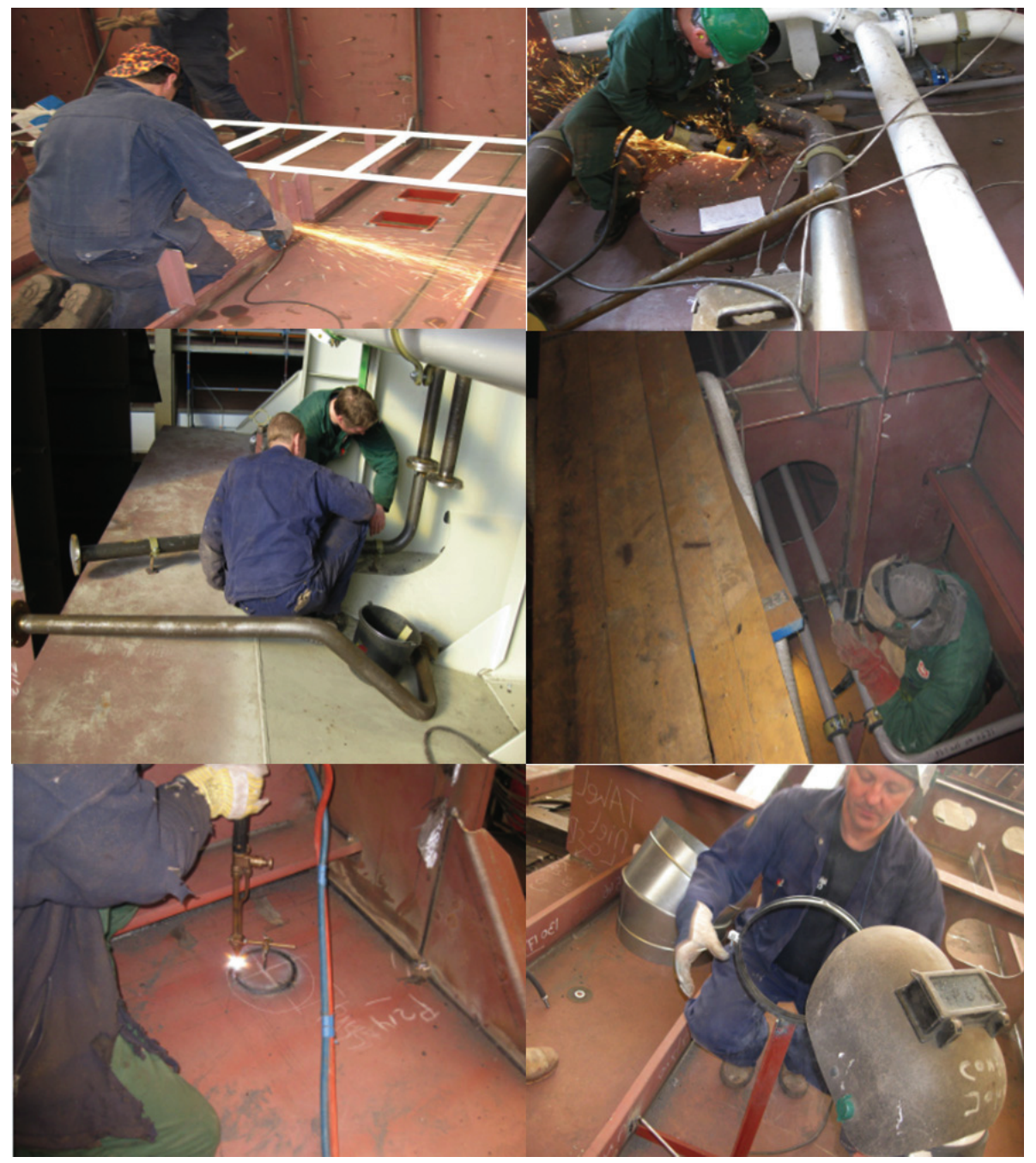

Figure 6.9: Examples of work position 


\section{Envelope of horizontal reaching, 20 - 30 years of age}

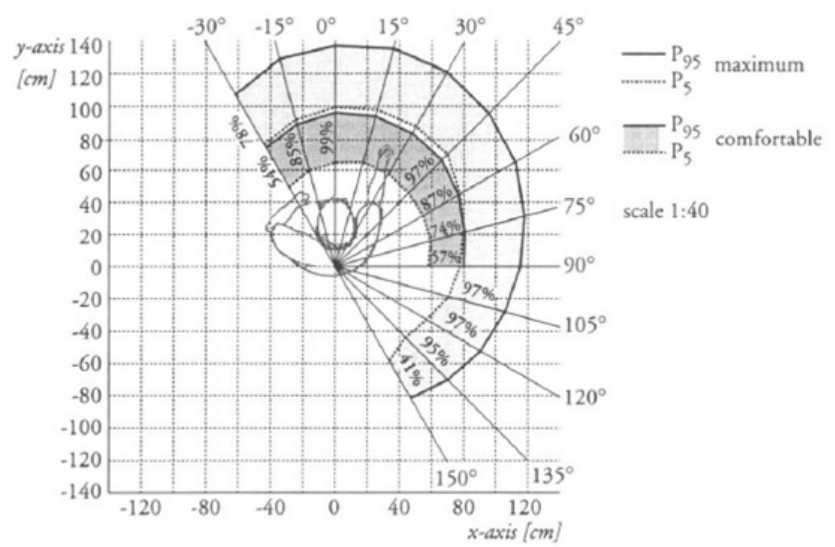

Note: Percentages in the graphic denote the proportion of the subjects ( $\mathrm{n}=117$ ) that reaches to related radius.

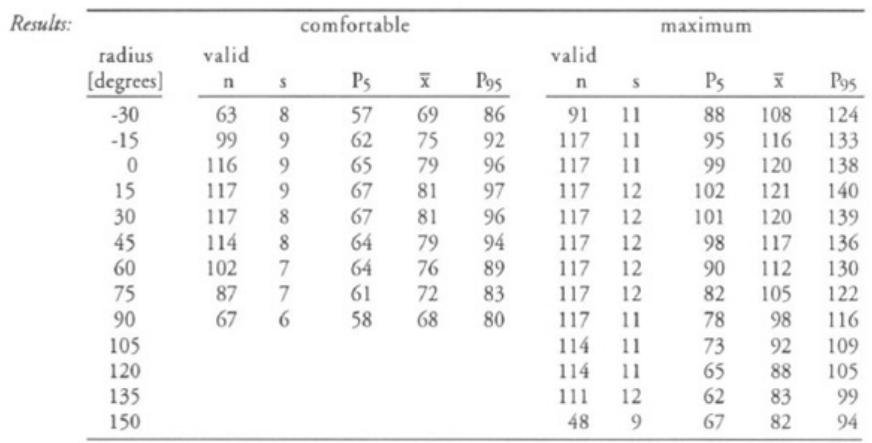

Figure 6.10: Envelope of horizontal reaching (reproduced from Steenbekkers (Steenbekkers and van Beijsterveld, 1998)) 
74 Automatic generation of assembly sequence for the planning of outfitting processes in shipbuilding

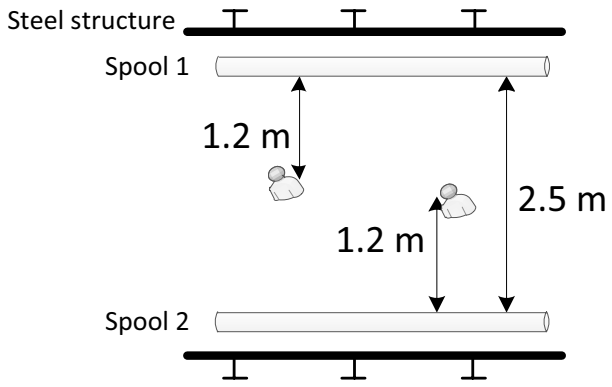

Figure 6.11: The minimum distance set as $2.5 \mathrm{~m}$

- Components' name for identification purpose only

- 3D coordinates of end and bend points

- Weight

- Material

- Diameter/Width

- Whether it is a penetrating piece or not

Together, they provide the initial input of the assembly sequence generation model.

\subsection{The detection of interferences between outfitting com- ponents}

Detecting the interferences between outfitting items serves to find the assembly order of components when only taking the position attribute into account. As we saw in chapter 4.2, automatic assembly may be studied from the point of view of disassembly. The backward process nicely fits the available starting data that invariably comes in the form of a more or less complete CAD model. Based on this assumption that the disassembly sequence is the reverse of a feasible assembly sequence, the interference matrix is used to detect the interferences among the $\mathrm{n}$ components in a section or compartment. It is a part of the Binary Matrix put forward by Dini and Santochi (Santochi and Dini, 1992). This section explores the application of the interference matrix in the research. We also specifically address a problem encountered during the application. It refers to endless height iteration. This section also describes how to solve it by trial and error. 


\subsubsection{The application of the interference matrix}

How can the interference matrix be applied to detecting the interferences and how these outfitting components could be disassembled in a collision-free order? As described in chapter 6.1, during the engineering stage the position of a component in a ship is described by the three dimensional coordinates of its end and bend points. These data can be accessed through querying the detailed engineering model.

The procedure to generate the disassembly order along the $+Z$ direction (from bottom of a ship to top of a ship) by interference matrix can be described as follows:

Step1: Build the matrix. Through translating spools along the $+Z$ direction, the interference matrix detects the spatial relationship between two components. This is done by the comparison of their $\mathrm{Z}$ coordinates. If there exists an interference between components $\mathrm{e}_{i}$ and $\mathrm{e}_{j}, \mathrm{a}_{i, j}=1$ and otherwise 0 . Set the initial disassembly order number to 0 ;

Step2: Find all the components $\mathrm{e}_{i}(1 \leq i \leq n)$ satisfying the condition: $\sum_{j=1}^{n} a_{i, j}=$ 0 , which implies none of the other components collide with component $\mathrm{e}_{i}$. These components are on the same height level (in the same height level group);

Step3: Record the components found in step 2, increment the disassembly order with 1 and set the disassembly order for $\mathrm{e}_{i}$ equal to the disassembly order number. This means that this batch of spools are the first ones to be disassembled;

Step4: Remove the above components from the matrix;

Step5: Rebuild the matrix with the remaining components;

Step6: Repeat steps 2-6 until no components remain.

By now, each component should have its own disassembly order and be categorized into a specific height level group. Figure 6.12 shows an example of 8 components. How the interference matrix along the $+Z$ direction detects the inference is shown in figure 6.13. The matrix on the very left side shows that component 2,3 and 7 are the first ones to be removed because all elements in row 2, 3 and 7 are 0 . It means no components collide with them along $+\mathrm{Z}$ direction. Thus, these components' disassembly order is 1 . Then, their absence makes available the disassembly of component 1 and 4 which have the disassembly order 2 . Next, the disassembly of component 8 becomes possible, followed by that of component 6 and finally, component 5 . At the end, the disassembly order of the 8 components is:

component $2,3,7 \rightarrow$ component $1,4 \rightarrow$ component $8 \rightarrow$ component $6 \rightarrow$ component 5

The same principle can be applied to detect the interferences when disassembling components along $-Z$ (from top of a ship to bottom of a ship) direction. Figure 6.14 shows the procedure to detect the interferences of the 8 components along the $-\mathrm{Z}$ direction. At the end, the disassembly order of the 8 components is:

component $1,2,5 \rightarrow$ component $3,6 \rightarrow$ component $8 \rightarrow$ component $4 \rightarrow$ component 7

The major difference is: when the translation is along the $+\mathrm{Z}$, the components 
76 Automatic generation of assembly sequence for the planning of outfitting processes in shipbuilding

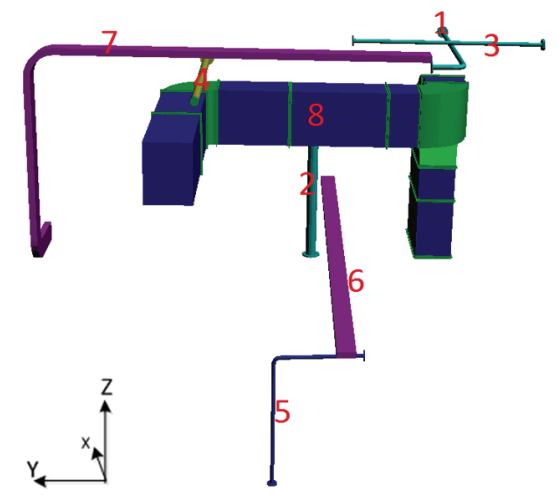

Figure 6.12: A test case with 8 components

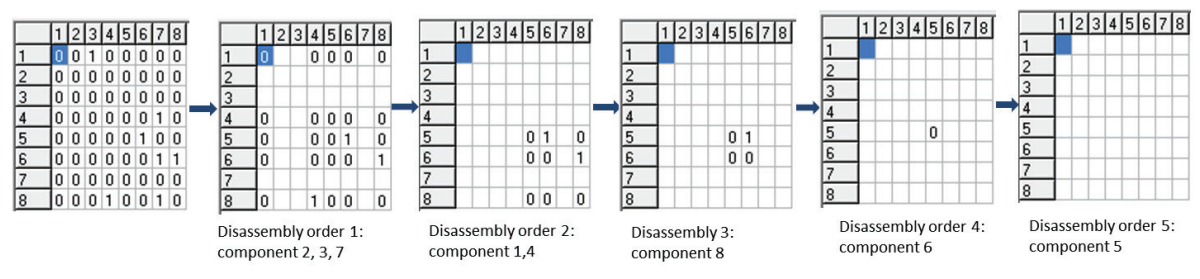

Figure 6.13: The procedure of interference detection along $+\mathrm{Z}$ direction

closer to the decks or ceilings are earlier to be disassembled. Their disassembly order is the same as their assembly order. When along the $-\mathrm{Z}$, the components further away from the decks or ceilings are earlier to be disassembled. Thus, the reverse of their disassembly orders are their assembly orders.

If the assembly sequence is derived by disassembling components along the $+\mathrm{Z}$ direction, it is called the 'Upward' version; on the contrary, it is called the 'Downward' version if along the $-\mathrm{Z}$ direction.

If two components have the same $\mathrm{Z}$ coordinates indicating the possibility to be assembled at the same time along $\mathrm{Z}$ direction, the same method can be used to detect their spatial relationship along either the $\mathrm{X}$ or $\mathrm{Y}$ direction to make sure the component closer to the bulkhead or other type of steel structure is assembled earlier? For example, in figure 6.15, spool 1 and 2 have the same $\mathrm{Z}$ coordinates but spool 1 is closer to the main transverse watertight bulkhead. During the assembly, workers prefer to install spool 1 first and then spool 2.

For a proper use of the disassembly method along the $\mathrm{X} / \mathrm{Y}$ direction by the interference matrix approach, the distance between spools and steel walls and bulkheads along the longitudinal/transverse direction should be accounted for. This requires using the steel structure 3D coordinates from the engineering model. This research has not included the steel structure information. In principle this is quite straight forward 


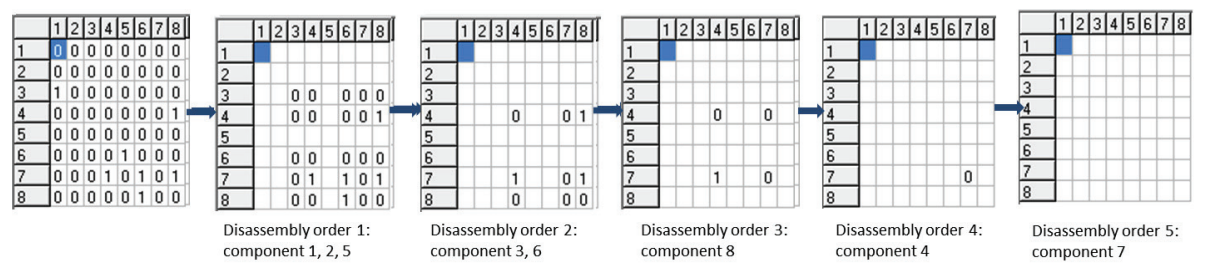

Figure 6.14: The procedure of interference detection along $-\mathrm{Z}$ direction

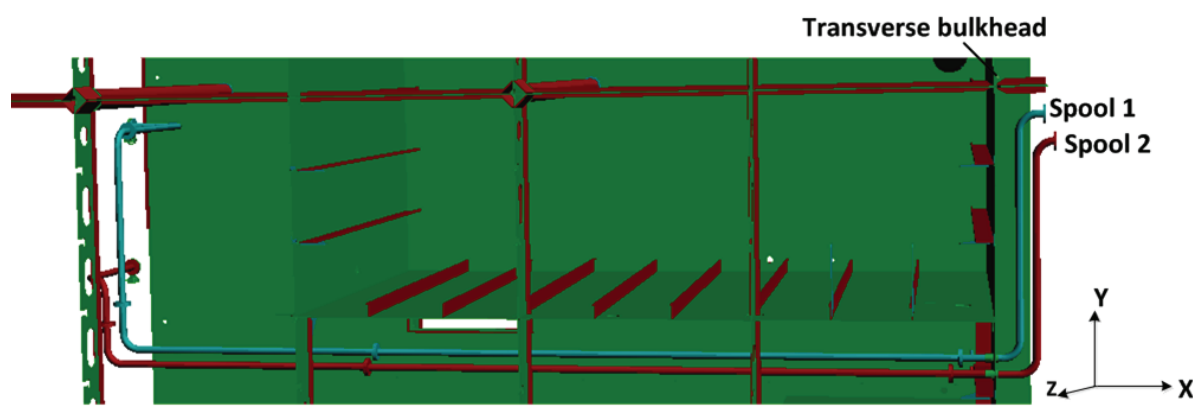

Figure 6.15: An example of two spools having the same $\mathrm{Z}$ coordinates but different $\mathrm{X}$ coordinates

but in practice it requires a large number of computations in order to cover for all potential structural/component situations. Indeed, a more general approach may be required if one takes account of the fact that workers may also insert components in a sequence of arbitrary directions. However, it is believed that once the distances (either along X or Y direction) between spools and their nearby structures are known, the interference between two components can be detected through the comparison of the distance, thus interference matrix along X/Y direction can also be established.

\subsubsection{Endless height iteration}

From the description in section 6.2.1, it is known that through the application of the interference matrix, spatial collisions along the direction $+Z$ between any two spools can be detected by the comparison of their $\mathrm{Z}$ coordinates. When collisions happen, the topology should be such that either one spool is above the other or the other way round. The matrix made 'black and white' judgments. However, some exceptions happen when two spools are interwoven with each other. In this case the model fails to discern which one is above the other. It causes the height iteration to run endlessly. So far, it was only encountered for HVAC ducts.

Figure 6.16 shows one of these examples. Three numbered HVAC ducts, duct 1 , 2 and 3 , are presented. Apparently, without the branch $1_{a}$ of duct 1 , there would have been no spatial collisions between them along the $\mathrm{Z}$ direction. Nevertheless, once the 
78 Automatic generation of assembly sequence for the planning of outfitting processes in shipbuilding

branch was added, not only its upper part is above duct 2 and 3 but its lower part is below them. Both duct 2 and 3 were half circled by this branch. The interference matrix established by the model looks like:

$$
A_{z}=\left[\begin{array}{cccc}
I D & d u c t 1 & \text { duct } 2 & \text { duct3 } \\
\text { duct } 1 & 0 & 1 & 1 \\
\text { duct2 } & 1 & 0 & 0 \\
\text { duct3 } & 1 & 0 & 0
\end{array}\right]
$$

Clearly no assembly order can be established in this case.
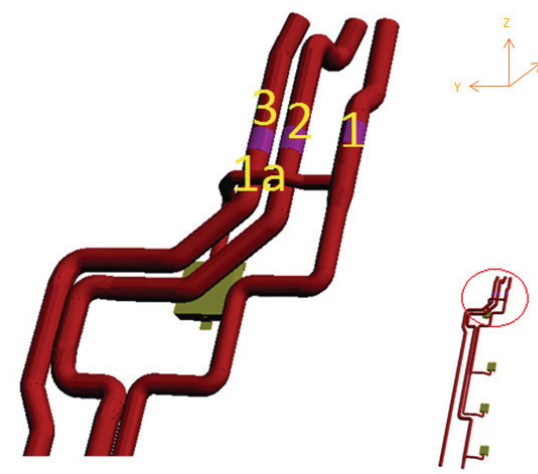

Figure 6.16: An example of the endless iteration problem

\section{Method 1: Further splitting spools}

After a conversation with a HVAC engineer, it was found that although such a case does not happen often, it indeed does exist. It happens more often especially in accommodation rooms where more air terminals are required to control the volume of treated air. Some of these terminals were added to the routed ducts very late in the engineering stage. Since the connection joints of spiral ducts are couplings with different radiuses, unlike assembling pipes, finding the space for it in between other ducts is somewhat easier. Thus, the case shown in figure 6.16 is regarded as being acceptable.

When the interweaving of spools happens, to distinguish which part of a spool is above the other and which part is below may help to untie the 'knot'. This idea led us back to a closer look at the original engineering model, where HVAC ducts are commonly presented as a whole piece without being split into 'spools'.

In the research, the data preparation model mentioned in section 7.1.2 is able to split a whole piece of HVAC duct into several pieces with a standard of $3 \mathrm{~m}$ each in length. This was based on how the assembly work in steel sections was carried out. 
However, interweaving cases may require further splitting of HVAC ducts in order to pinpoint the spatial relationship between different parts of a spool and another spool. Thus, a further splitting of the spools into smaller pieces could be attempted $(2 \mathrm{~m}$, $1.5 \mathrm{~m}, 1 \mathrm{~m}$ and $0.5 \mathrm{~m}$ for example).

However, it was found that the endless height iteration still occurs after such a further split because some parts of a spool were still half circled by couplings. These couplings are a kind of finished product and cannot be further divided. Meanwhile, this further split also caused a drastically increased number of spools. These spools are too small and violate model assumptions of mounting time, number of supports, etc.

\section{Method 2: Setting a rule}

Since this interweaved design was judged acceptable, workers should be able to find a way to install these ducts, following some implicit and unknown rules. Further field observations and interviews showed that workers are inclined to assemble 'complicated' spools first. Their definition of a 'complicated' spool is one that has many couplings. This is because increased positioning, drilling and wrapping work is associated with a 'complicated' spool, and the execution of these procedures takes some space and a longer time. Thus, the workers' logic is that once the complicated ducts are installed, it is much easier to find space for the simple ones. Based on their description, an additional rule was set up, which is if two spools are interwoven with each other, the one with more couplings should be installed earlier.

Since the number of bends of a certain spool has already been derived in the data preparation model, the application of this rule in a computerized manner is easy. The new procedure is:.

Step 1: Establish the interference matrix, including all spools;

Step 2: Find where the endless iteration happens: if both $\mathrm{e}_{i j}$ and $\mathrm{e}_{j i}$ have the value ' 1 ', the spool $i$ and $j$ are interwoven with each other;

Step 3: Compare which spool, i or j, has more couplings; if spool i has more, the value of $\mathrm{e}_{i j}$ remains ' 1 ' and $\mathrm{e}_{j i}$ becomes 0 , because spool $\mathrm{i}$ will be disassembled later and assembled earlier than spool $\mathrm{j}$. If they have equal number of couplings, in reality it does not matter which one is assembled earlier. In this case, the value of $\mathrm{e}_{i j}$ is chosen to remain ' 1 ' and $\mathrm{e}_{j i}$ is set to 0 .

\subsection{The relative importance of each physical attribute}

The possibility to quantify the impact of each physical attributes in the determination of the assembly order and the availability of interference relationships of components lead to the consideration of 'the relative importance'. The contribution of each physical attribute to the determination of the assembly sequence varies based 
80 Automatic generation of assembly sequence for the planning of outfitting processes in shipbuilding

on the particular situation and is subjectively influenced by expertise from experts. One attribute may require the component to be assembled earlier and another later. Thus, we adopt the AHP method to decide the relative importance of each physical attribute which is measured by weighting coefficients. This method has unique advantages when importance levels of the decision are difficult to quantify or compare and where communication among experts from different disciplines is hindered by their different specializations, terminologies, or perspectives (Bhushan and Rai, 2004). Then, we rank components based on their physical attributes and weighting coefficients to determine their mounting orders.

\subsubsection{AHP method}

AHP is a methodology where various elements are evaluated based on pairwise comparisons of the elements. In making the comparisons, the decision makers can use concrete data about the elements, or they can use their even more subjective judgments about the elements. It is the essence of the AHP that human judgments can be used in performing the evaluations (Saaty, 1980).

As the AHP is based on subjective judgments of human perception, it must be checked for consistency. To measure how consistent the judgments have been relative to samples of purely random judgments, Saaty (Saaty, 1980) proposed what is called the consistency index $(\mathrm{CI})$. If the value of $\mathrm{CI}$ is smaller or equal to $10 \%$, the inconsistency is acceptable because a human is not always consistent. If it is greater than $10 \%$, we need to revise the subjective judgments. The general description of the AHP calculation process, including the pairwise comparison, making the comparison matrix, computing the priority vectors and calculating the Consistency Ratio can be found in 'Analytic Hierarchy Process AHP Tutorial' (Teknomo, 2006).

During the field study, interviews were carried out to ask foremen and workers to make a pairwise comparison of the relative importance of the five physical attributes, using the scale shown in figure 6.17. The value 1 indicates that two attributes are equally important. The value 3 means one is slightly more important than the other. The larger the value, the higher importance level of one versus the other. The results of these interviews can be found in appendix A.

The results of the analysis are shown in figure 6.18. The CI of this calculation was $4.79 \%$, less than the maximum allowed inconsistency of $10 \%$. Therefore, the degree of inconsistency is acceptable and the result of AHP is considered reliable and valid.

The data in figure 6.18 shows that when workers decide on the assembly sequence on site, they are of the opinion that the position of spools in the steel structure takes the dominant role (0.434). The next attribute of importance is the penetration $(0.34)$. The third important attribute is the weight (0.104), followed by the size and material. The relative importance percentages one-to-one reflect the weighting coefficients. 


$\begin{array}{ll}\text { Value } & \text { Level of importance } \\ 1 & \text { Equally important } \\ 3 & \text { Slightly more important } \\ 5 & \text { Strongly more important } \\ 7 & \text { Very strongly more important } \\ 9 & \text { Extremely more important }\end{array}$

Figure 6.17: Scale to measure the level of importance

\subsubsection{Ranking}

In chapter 6.1, each attribute of the component leads to its ranking in the assembly order. Take 8 components in the figure 6.12 as an example. The rankings of each component resulting from each attribute are displayed in table 6.1. The column of 'Penetration' is used to check whether it is a penetrating piece. If components have different penetration values, care must be taken that their ranking values are according to the number of components. This is necessary to be ensured that the weighting of e.g. position ranking (which varies with the number of components) and that of penetration remain proportional. Therefore the ranking of components with penetration $=1$ is set to the number of components. For non-penetration pieces the value is set to zero.

For the position ranking, several spools can be assembled simultaneously because of the absence of collisions and should have the same position ranking. We take the average of the assembly position value (the value in the brackets in the column of 'Position Ranking' in table 6.1) of the components that can be assembled at the same time as their position ranking. Components to be assembled earlier have a higher position ranking value. For example, the assembly position values of components 2 , 3 and 7 respectively are 6,7 and 8 and their position ranking equals $(6+7+8) / 3=7$.

The same principle is also applied to material ranking. In most cases more than one component has the same material type and should have the same position ranking. For example, in table 6.1, apart from component 8 which is a rectangular duct, the other components are steel pipes. Thus, the average value is calculated and assigned as material ranking to each component. 
82 Automatic generation of assembly sequence for the planning of outfitting processes in shipbuilding

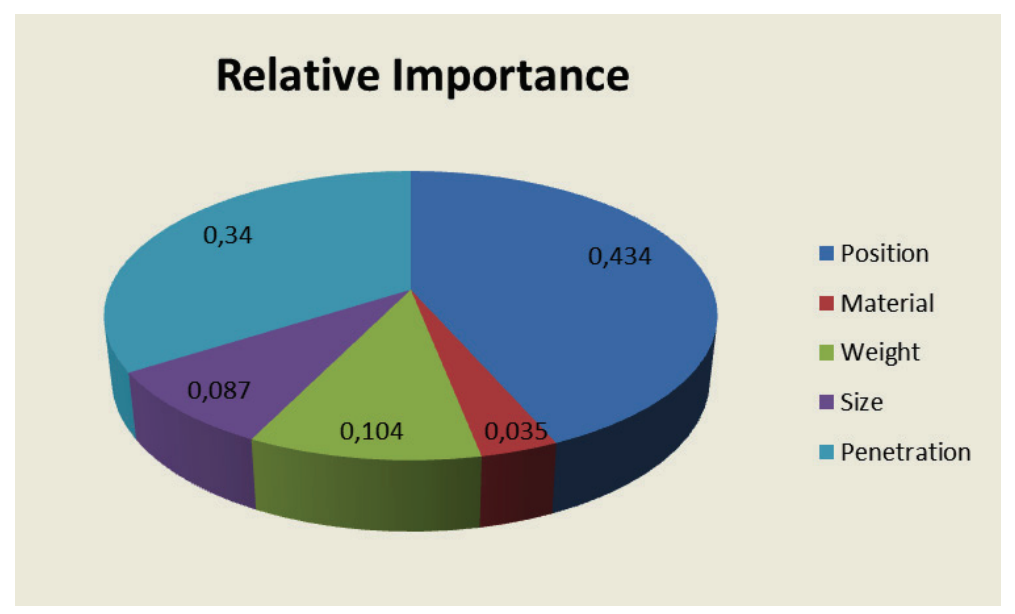

Figure 6.18: Relative importance of physical attributes

Table 6.1: Assembly ranking of 8 components

\begin{tabular}{llllllll}
\hline $\begin{array}{l}\text { Component } \\
\text { ID }\end{array}$ & $\begin{array}{l}\text { Position } \\
\text { Ranking }\end{array}$ & $\begin{array}{l}\text { Material } \\
\text { Ranking }\end{array}$ & $\begin{array}{l}\text { Weight } \\
\text { Ranking }\end{array}$ & $\begin{array}{l}\text { Size } \\
\text { Ranking }\end{array}$ & Penetration & $\begin{array}{l}\text { Total } \\
\text { score }\end{array}$ & $\begin{array}{l}\text { Final } \\
\text { Ranking }\end{array}$ \\
\hline 1 & $4,5(4)$ & $5(2)$ & 3 & 3 & 0 & 2,701 & 4 \\
2 & $7(6)$ & $5(3)$ & 5 & 6 & 0 & 4,255 & 7 \\
3 & $7(7)$ & $5(4)$ & 1 & 1 & 0 & 3,448 & 6 \\
4 & $4,5(5)$ & $5(5)$ & 4 & 4 & 0 & 2,892 & 5 \\
5 & $1(1)$ & $5(6)$ & 2 & 1 & 0 & 0,948 & 1 \\
6 & $2(2)$ & $5(7)$ & 6 & 7 & 0 & 2,32 & 2 \\
7 & $7(8)$ & $5(8)$ & 7 & 7 & 0 & 4,593 & 8 \\
8 & $3(3)$ & $1(1)$ & 8 & 5 & 0 & 2,604 & 3 \\
\hline
\end{tabular}

Since the relative importance of each physical attribute has been measured by weighting coefficients, each component gets its own total score by the formula:

Total Score $=\sum_{i=1}^{5}($ Assembly Ranking $i \times$ Weighting Coefficient $i)$

For example, in table 6.1, the total score of component 1 is:

$$
4.5 \times 0.434+5 \times 0.035+3 \times 0.104+3 \times 0.087+0 \times 0.34=2.701
$$

Consequently, the assembly order of all components within a certain steel section can be obtained by sequencing them according to their scores. The higher the total score is, the earlier the corresponding component is assembled. In table 6.1, the total scores are listed and the 8 components are sequenced according to the final ranking, which is: 
component $7 \rightarrow$ component $2 \rightarrow$ component $3 \rightarrow$ component $4 \rightarrow$ component $1 \rightarrow$ component $8 \rightarrow$ component $6 \rightarrow$ component 5

This ranking method combines the factors of the physical attributes and their importance level to generate sequences. In terms of technical aspects, the results are feasible. However, the derived assembly sequences require the components to be installed sequentially. This is not realistic on the work site where workers often install several components at the same time if workspace allows it. How to make the assembly sequences more reasonable with the consideration of minimum work distance will be discussed in chapter 8 .

\subsection{Summary}

Through the analysis of physical attributes of components, the answer to each question put forth in the beginning becomes clear and is as follows:

- Five physical attributes are chosen: position, material, weight, size, penetration and minimum work distance. The determination of their ranking has also been quantified.

- The interference matrix has been applied to detect the inferences amongst the components, trying to create a collision-free disassembly path.

- Weighting coefficients, resulting from the calculation by AHP method, are used to measure the relative importance of each attribute. The final rankings of components for their assembly sequence are available, considering both the physical attributes and their importance level.

These answers form the initial input of the assembly sequence generation model. What kind of data is needed in the detailed engineering 3D model is also known. The data collection is introduced in the next chapter. 



\section{Chapter 7}

\section{Data Collection and Preparation}

To be able to generate the assembly sequence automatically, obtaining the necessary information through the logic reasoning to subsequent deduction of sequence should be done by a computer model facilitating reduction of the workload for a planner as much as possible. Chapter 6 gave an overview of the data definition, i.e. what types of data for the creation of automatic assembly sequence generation model are needed. UML class diagram is applied to express the operations and attributes of different data classes and to show their interrelationships. It can be found in the appendix C. This chapter mainly presents what data can be obtained from which sources. The reasons why some data currently are not available will be explained and the solution of how to get them in the future will be recommended. In order to make the method more universal, some assumptions are made during data collection, which will be described.

\subsection{Data sources}

\subsubsection{Raw data}

In the ideal situation, the physical attributes of a certain outfitting component which may influence its mounting sequence, as described in chapter 6 , should be included in 3D model. Of course, such geometric information of components should be precise and detailed enough to allow them to be produced and installed. Workers should need no other information to complete the job. Thus, the detailed engineering model is chosen as the resource for input data.

It provides the basis for the desired production drawings and instructions (because that is what the engineering process is meant to provide) in time for steel pre-fabrication, fabrication of pipes and other parts, steel structure construction, preoutfitting of sections, ship erection and the outfitting of the erected ship. Another reason to choose the detailed engineering model as the data source is the low prob- 
86 Automatic generation of assembly sequence for the planning of outfitting processes in shipbuilding

ability of late fundamental modifications. According to the staff, 6 weeks before cutting the plates of a section, all production information of that section is available in the detailed engineering model. This, to a great extent, helps to reduce the chance of modifying the assembly sequence at a later stage caused for example by the replacement of crucial equipment or rerouting of pipes or cable trays.

From the planning aspect, the detailed engineering model becomes available in a rather late stage just before work preparation starts. To use the detailed engineering model as an input implies that the results of our method cannot be used during the initial planning stage unless the initial information of components is available. According to Asmaras' work (Asmara and Nienhuis, 2006, 2007, 2008; Asmara, 2012), his method allows to generate pipe routes during an earlier stage of engineering. As soon as the initial physical attributes of pipes (and other parts) are available, given the functionality of our model to further split pipes into spools, our method can also be applied in the initial planning stage. Thus, to a certain extent, it can help to reduce the uncertainties and make the planning more reliable even at the very early stage of a shipbuilding project. The method may then provide a more reliable indication of the duration time of outfitting certain sections when making a pre-/outfitting plan for the next shipbuilding project.

When looking into the detailed engineering model of a real case, it turns out that the model contains in principal all the geometric information that is needed, including the size, position, weight, diameter, and material of components. Extracting and manipulating the data from the engineering model requires a separate module. Indeed, each CAD application requires its own specific version of this module. For our purposes, we programmed in Delphi. Delphi was chosen as the programming language for the following reasons:

- Allowing fast and high-level/abstract programming and generating fast optimized code.

- Operating in an industry standard environment and allowing any industry to carry it out in their respective fields of production.

- Being object oriented, it is easier to make a coupling with existing systems and to extend it in future research without limitation.

- Availability of local knowledge.

A proper export format from the detailed engineering model needs to be chosen to make sure it fulfills the requirement of the Delphi software. In the shipbuilding industry, different CAD systems, such as Tribon, Nupas-Cadmatic or NX, are used for design work. Different CAD packages use different types of database structures to store the information of a part in a CAD file. The Tribon model was selected because the real cases were based on Tribon. 


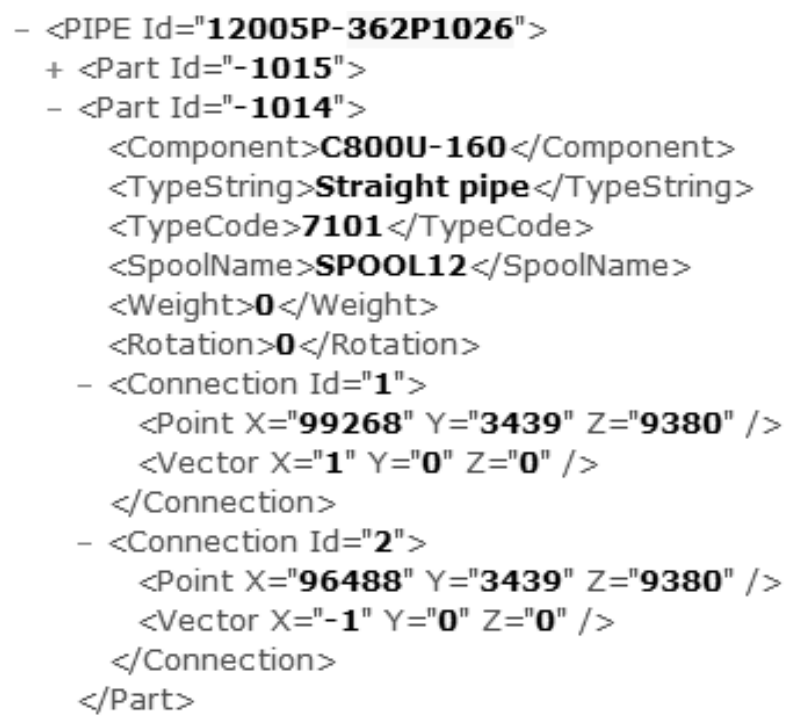

Figure 7.1: An example of xml file produced by Tribon

Regrettably, data exchange format (DXF) cannot be selected as the format to extract data from a Tribon model. DXF is a widely used standard and is based on geometry information and primarily used by 'general purpose CAD systems' for data exchange regarding drawings and solid models. Since it entails the collection of all points (vertices) and faces of components without knowing which faces belong to which components, it is sufficient for generating the drawings of components. However, it is not readable since there is no simple way of associating attribute data with points, lines, and polygons. Besides, although DXF files are usually text files (though some are binary) and can be viewed, and in theory, edited in a text editor, in practice, they are not easy to understand.

Therefore extensible markup language (XML) was selected to be used as an exchange mechanism between Tribon and the Delphi software. In July 2003, Tribon Solutions promoted the standardization of the shipbuilding CAD and CAM data exchange using XML to become a standard within the shipbuilding industry (Tann and Shaw, 2007). The XML data is structured and has a tree shape. Even complex relationships in the tree structure are clear in its format. The codes in XML are easily legible to people who have had no formal introduction to it, because it is all written in simple plain text and in a human readable language. Moreover, XML is very easy for the Delphi programming language to be parsed and reorganized. Figure 7.1 is an example of a part of the XML file used to describe part of a Tribon model in the detailed engineering stage and figure 7.2 is the visual representation of its corresponding solid component, a HVAC duct.

For this particular component, as shown in figure 7.3, the detailed engineering 
88 Automatic generation of assembly sequence for the planning of outfitting processes in shipbuilding

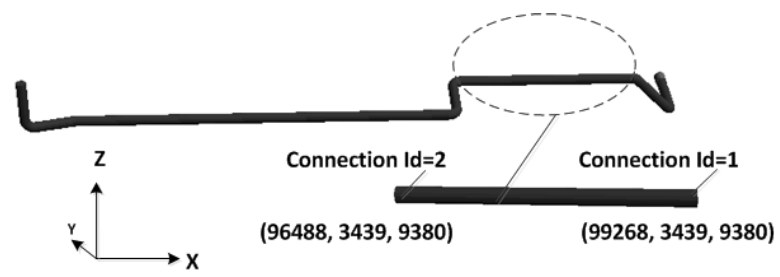

Figure 7.2: A piece of HVAC duct corresponding to the XML file in figure 7.1

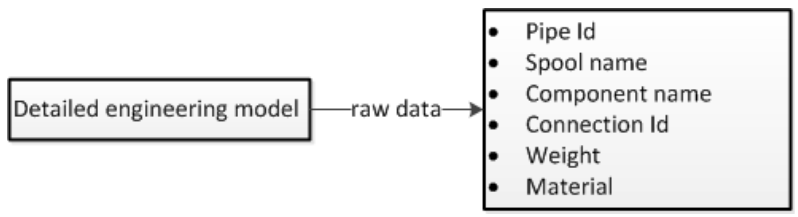

Figure 7.3: Raw data from the detailed engineering model

model provides the data of pipe ID, component name, spool name, connection ID, weight and material. Pipe ID is the specific name of a pipe, cable tray or a duct in a ship. Under the class of pipe ID, there may be several instances of 'spool name' and it describes what spools belong to this item. The purpose of using both pipe ID and spool name is to distinguish each spool from the rest for its unique fabrication and handling in the later stages. Component name is used to distinguish the type of this component, i.e. whether this component is pipe, cable tray or duct. Connection ID illustrates the position of a spool piece by means of its three dimensional coordinates (of its beginning and end points). Weight and material plainly describe the weight and material of each spool.

Nevertheless, not all data indispensable for our sequence generation model can be directly obtained from the detailed engineering model. Examples are the length of each spool and the number of couplings per piece of HVAC duct. This leads to the necessity of a data preparation model, which is used to extract and/or derive the needed information from the XML file. It will be introduced in the following subsection.

\subsubsection{Data preparation model}

XML data from the Tribon model can be read by the code written in Delphi. To reorganize these data in a way that suits the assembly sequence generation model to be described in chapter 8 still needs some exploration. Moreover, data available in an XML file is not sufficient for the requirements of physical attributes of each component described in chapter 6; more information should be added in. Hence, the data preparation model is applied as a bridge. It is not only able to read original XML data and output the reorganized data in a suitable layout, but also to provide 


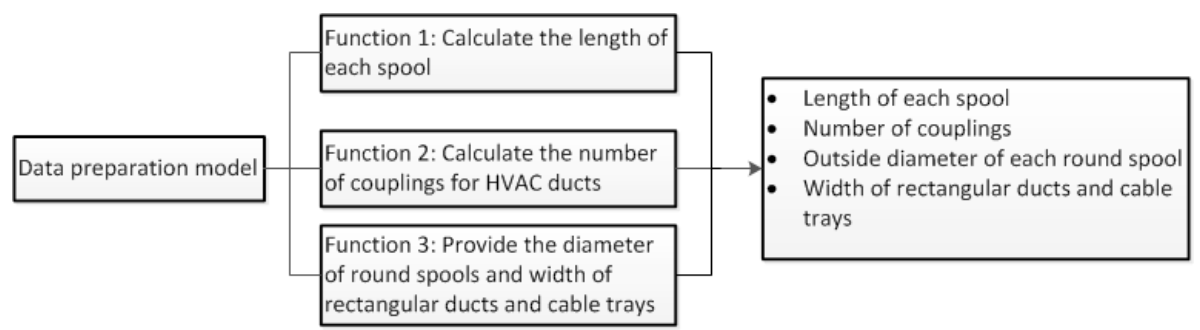

Figure 7.4: Functions of data preparation model

a platform allowing more data to be added in. Figure 7.4 shows that it has three functions. Each function is explained as follows.

1. Calculate the length of each spool for two purposes:

- The length is a standard parameter indicating the need for production purposes to split pipes into spools.

In this report, we also apply the term, spool, to cable trays. In reality outfitting processes are decomposed into a set of tasks up to an appropriately detailed level. Pipes, HVAC ducts and cable trays are assembled spool by spool on the work site. Thus, the engineering drawing is expected to provide geometric information of components on the spool level.

However, in the original engineering database, often only pipes and cable trays are split up into spools. This is because pipe spools consist of tubes and flanges, welding corners, adapters, etc. Cable tray spools consist of bends and straight cable tray. They need a certain time for fabrication before they are transported to the assembly site. Therefore pipe and cable tray spools are mostly clarified in the model in advance. Note that this does not apply to thin pipes (diameter is less than $12.5 \mathrm{~mm}$ ) and strips (used to carry one or two cables) which are prepared on site.

HVAC ducts however, are often modeled as a whole piece without being split in the detailed engineering model. Figure 7.2 is an example of one piece of HVAC duct in the model. It has not been split into several spools which are suitable for workers to assemble. Their standardized products are directly transported to the work site from the warehouse. Workers make decisions of how to split them on the site based on their routing conditions shown on the drawings. The standard length of a duct is $3 \mathrm{~m}$ or $6 \mathrm{~m}$. According to the staff, in the maritime industry, they prefer to use $3 \mathrm{~m}$ in length as a standard duct. Figure 7.5 is an example of cutting HVAC ducts. Thus, spool-splitting is not included for HVAC in the original engineering database.

Taking the HVAC duct shown in 7.2 as an example, the method to split a com- 
90 Automatic generation of assembly sequence for the planning of outfitting processes in shipbuilding

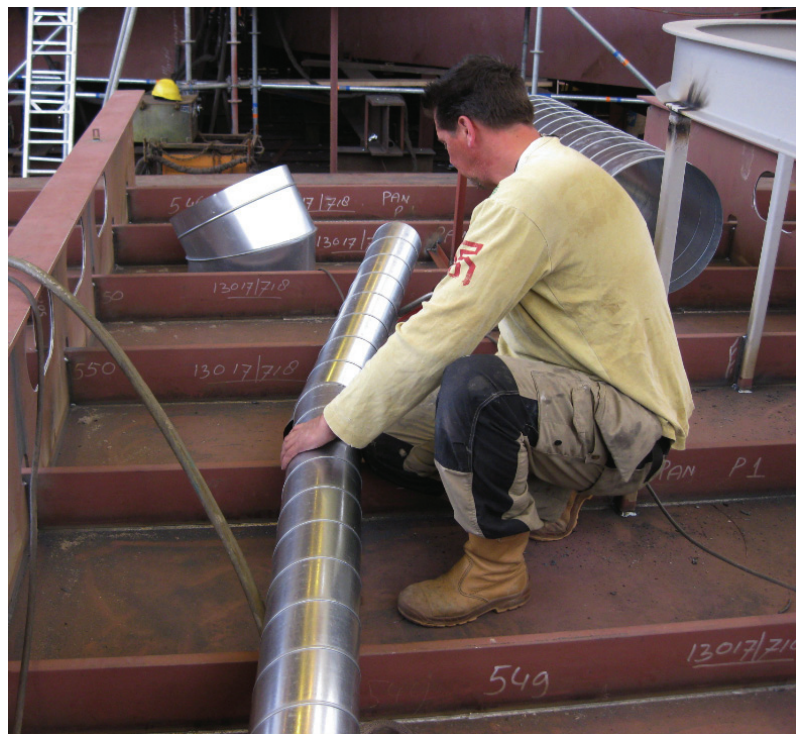

Figure 7.5: An example of cutting HVAC ducts

plete piece of HVAC duct into several spools with the standard of $3 \mathrm{~m}$ in length is as follows. The geometrical information of this duct has been displayed in figure 7.6.

Step 1: Find the starting and ending points of a piece of HVAC duct by counting the frequencies of the repetition of the 3D coordinates, because they are put in random order in the XML file. It is logical that the $3 \mathrm{D}$ coordinates of the starting and ending points should only be counted once since they do not show up both as starting and ending point. Figure 7.6 has marked out the starting and ending point of this duct.

\begin{tabular}{|c|c|c|c|c|c|c|c|c|c|c|c|c|c|}
\hline \multirow[t]{2}{*}{2} & \multicolumn{4}{|c|}{ 그 7719-12005P-362P1026 } & $\Gamma$ Sorted & \multicolumn{3}{|c|}{ Change SPOOL id } & CountCouplings & \multicolumn{3}{|c|}{ Sort Pieces } & \multirow{2}{*}{$\frac{\sqrt{3000} \text { Max. }}{\text { Tot.L }}$} \\
\hline & Type & \begin{tabular}{|l|} 
Spool \\
\end{tabular} & P1X & P1Y & P1Z & $\mathrm{P} 2 \mathrm{X}$ & $\mathrm{P} 2 \mathrm{Y}$ & $\mathrm{P} 2 \mathrm{Z}$ & Con. Type & 0 & 0 & L & \\
\hline .1003 & Component & SPOOL12 & 99973 & 4604 & 8740 & 99973 & 4404 & 8740 & Couplings & 1 & 2 & 200.00 & 200 Starting point \\
\hline-1012 & Straight pipe & SPOOL12 & 99973 & 4404 & 8740 & 99973 & 3599 & 8740 & Pipes & 2 & 2 & 805.00 & 1005 \\
\hline-1006 & Component & SPOOL12 & 99973 & 3599 & 8740 & 99860 & 3439 & 8853 & Couplings & 2 & 2 & 226.14 & 1231.14 \\
\hline .1005 & Straight pipe & SPOOL12 & 99860 & 3439 & 8853 & 99379 & 3439 & 9334 & Pipes & 2 & 2 & 680.24 & 1911.38 \\
\hline .1004 & Component & SPOOL12 & 99379 & 3439 & 9334 & 99268 & 3439 & 9380 & Couplings & 2 & 2 & 120.15 & 2031.53 \\
\hline .1014 & Straight pipe & SP00L12_A & 99268 & 3439 & 9380 & 96488 & 3439 & 9380 & Pipes & 2 & 2 & 2780.00 & 2780.00 \\
\hline-1007 & Component & SPOOL12_B & 96488 & 3439 & 9380 & 96328 & 3279 & 9380 & Couplings & 2 & 2 & 226.27 & 226.27 \\
\hline-1001 & Straight pipe & SP0OL12_B & 96328 & 3279 & 9380 & 96328 & 2553 & 9380 & Pipes & 2 & 2 & 726.00 & 952.27 \\
\hline-1008 & Component & SPOOL12_B & 96328 & 2553 & 9380 & 96168 & 2393 & 9380 & Couplings & 2 & 2 & 226.27 & 1178.54 \\
\hline .1010 & Straight pipe & SP0OL12_C & 96168 & 2393 & 9380 & 90352 & 2393 & 9380 & Pipes & 2 & 2 & 5816.00 & 5816.00 \\
\hline-1013 & Component & SP0OL12_D & 90352 & 2393 & 9380 & 90309 & 2387 & 9380 & Couplings & 2 & 2 & 43.42 & 43.42 \\
\hline .1015 & Straight pipe & SP0OL12_D & 90309 & 2387 & 9380 & 89738 & 2234 & 9380 & Pipes & 2 & 2 & 591.14 & 634.56 \\
\hline .1009 & Component & SP0OL12_D & 89738 & 2234 & 9380 & 89584 & 2193 & 9540 & Couplings & 2 & 2 & 225.83 & 860.39 \\
\hline .1002 & Straight pipe & SP0OL12_D & 89584 & 2193 & 9540 & 89584 & 2193 & 10188 & Pipes & 2 & 1 & 648.00 & 150839 Ending point \\
\hline
\end{tabular}

Figure 7.6: The geometrical information of the duct in data preparation model 


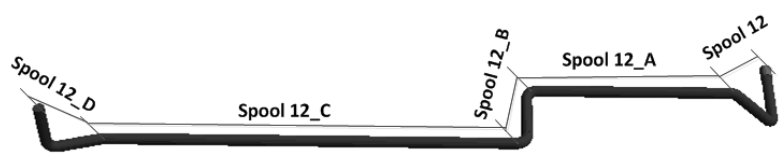

Figure 7.7: An example of splitting a piece of HVAC duct into 5 spools

Step 2: Following the main line indicated by the starting and end points, sequence the segment of a piece of duct according to their 3D coordinates. The type of the segment could be a straight pipe, coupling or cable tray.

Step 3: Calculate the length of each segment. If the length of a segment is longer than $3 \mathrm{~m}$, this segment is regarded as one separate spool even though it is longer than $3 \mathrm{~m}$. For example, in figure 7.6, the length of a segment is 5816 $\mathrm{mm}$. It is treated as one spool with the name spool12_C.

Step 4: Calculate the length from the starting point of the first segment to the end point of the current segment, called 'Total length'. In figure 7.6, spool 12 is the first spool and consists of 5 segments (three couplings and two straight pipe pieces).

Step 5: If the total length counted from the first segment up to and including the current segment is longer than $3 \mathrm{~m}$, the current segment should become the first segment of the next spool and a new spool name is assigned. In figure 7.6, the segment with id -1014 is the starting piece of the next spool spool12_A.

Step 6: Repeat step 4-5 until the last segment has been categorized .

After the splitting, the whole piece of HVAC duct with the length of $13.3 \mathrm{~m}$ consists of 5 spools as shown in figure 7.7. Note that splitting thus always occurs at bends or after a straight line segment with the length close to $3 \mathrm{~m}$ or more than $3 \mathrm{~m}$.

- The length of the spool is also necessary to estimate the mounting time of a pipe spool and a cable tray spool.

To be able to estimate the duration of outfitting a section is one of the research objectives described in chapter 1.3. In order to achieve this goal, the geometric attributes of components that decide their mounting time have been investigated. Field observations during one day per week on the work site have been carried out for more than one and a half years. During the observations, the assembly activities and their duration were recorded and can be found in appendix B.

For pipe spool and cable tray mounting, the length of the spools decides the number of spool supports. As observed, preparation work, measurement, connection spools, support-making and welding, and mounting spools are the main 
92 Automatic generation of assembly sequence for the planning of outfitting processes in shipbuilding

steps to mount a spool. The step of making supports for a spool occupied the largest part of its mounting time. For pipe supports, it requires workers not only to make supports in the warehouse, but also beforehand to observe the pipes to be installed and figure out the number of needed supports and their positions. For cable trays, although supports are made on the worksite, measuring, cutting, grinding and welding them still takes most of the cable tray mounting time. Thus, the mounting time of a spool is related to the number of supports it needs.

According to Pipe Hanger and Support Recommended Specifications (Erico, $2009)$, for pipes with a 10 feet $(3.0 \mathrm{~m})$ maximum spacing, there is a minimum of one hanger per pipe section close to the joint and also one at a change of direction and/or branch connection. From this point of view, the length of the spools decides the number of supports.

2. Calculate the number of couplings of HVAC ducts.

The purpose of calculating the number of couplings is to find the mounting time of a piece of HVAC duct. In HVAC system installation, flexible ducts should be joined by a metal sleeve, collar, coupling, or coupling system. Although the time spent on the supports-making to connect ducts with the steel structure is necessary, it is insignificant compared to the mounting time spent on the installation of couplings. This is also due to the fact that HVAC ducts are light weight and only transport air. Figure 7.8 illustrates the large number of couplings in the HVAC ducting. Our field study and experience from HVAC installation foremen shows that the installation of couplings is the most time-consuming part due to the strict requirements during their complicated installation procedures. These are for example:

- At least 2 inches $(50 \mathrm{~mm})$ of coupling must extend into the inner core of a duct while allowing 1 inch attachment area on coupling for the application of tape.

- The inner core of a duct should be mechanically fastened to couplings, using screws ( 3 screws for ducts up to 12 inch diameter, and 5 screws for ducts over 12 inch diameter) equally spaced around the diameter of the duct. Such a connection should also be able to capture the wire coil of the inner liner of the duct if it is insulated.

- The inner core should be sealed to the fitting with mastic or approved tape. Tape used for sealing the inner core should be applied with at least 1 inch of tape of the duct, 1 inch of tape on the coupling, and wrapped at least three times.

More couplings mean more connection work. Thus, the mounting time of a piece of duct largely depends on how many couplings the duct has. The estimation of such mounting time can be found in appendix B. 


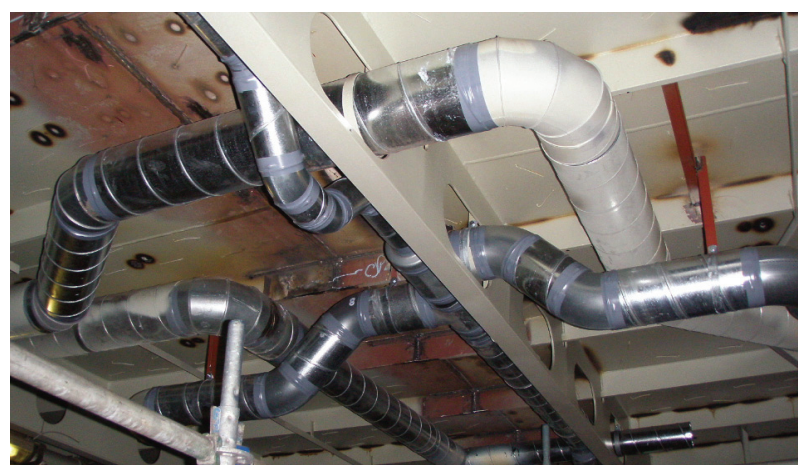

Figure 7.8: Ducts with couplings

3. Provide the diameter of round spools and the width of rectangular ducts or cable trays.

Since size is one of the important physical attributes of a component to decide its mounting order, as described in chapter 6 , the diameter of round spools and the width of rectangular ducts/cable trays are chosen as a criterion when the comparison of the size of spools is made. So far, the model includes the diameter of three types of round spools- steel pipes, plastic pipes and insulated or non-insulated Spiro-duct. In the original XML file as we use it, the diameter and the width information were not expressed directly but implied in the description of a name of component. For example, according to the name convention:

- If the name of a component is ' $\mathrm{C} 800-80$ ', C800 represents it as a spiral duct and 80 after '-' its nominal diameter;

- If the name is 'C820-65-85', 'C820' signifies a rectangular air duct and '-65$85^{\prime}$ means the width of the duct is $850 \mathrm{~mm}$ and the height is $650 \mathrm{~mm}$;

- If the name is 'C700-100', 'C700' indicates a piece of cable tray, '-100' means the width of the cable tray is $100 \mathrm{~mm}$.

Engineering departments in different shipyards each have their own name convention or even use another way to describe the information on the diameter. By this aspect, the data preparation model will not be compatible with different ways to express the diameter and the width. It will need to be adapted for different companies.

\subsubsection{Equipment database}

For equipment the location and the connection points with other spools or equipment play an important role in their mounting order. This is because location information is used to detect the spatial relationships between a piece of equipment and its surrounding outfitting components. Connection points help to detect to which spool a certain 
94 Automatic generation of assembly sequence for the planning of outfitting processes in shipbuilding

piece of equipment is connected. In the CAD model, equipment is often expressed as a rectangular boundary box. Since all equipment-related data came from different sources, they were put together in the database. The coordinates of the boundary box, the section ID and the mounting time of equipment were input manually.

To establish the equipment database, the following four points need to be emphasized:

1. A list of equipment

Equipment is not named according to its locations but by which functional system it belongs to. To know what equipment is in which steel section requires some data manipulation. Based on the two diagonally opposite corner points of the bounding box of each steel section and bounding box of each piece of equipment, a reasonable assumption can be made. By filtering for the boundary boxes of all equipment that lie within a certain section, a list of equipment within that section can be generated.

\section{Coordinates of eight points of the equipment boundary box}

When the alignment of equipment is along the three Cartesian axes of a ship, the boundary box of a piece of equipment is sufficiently precise to express its location, such as EP1 (equipment 1) in figure 7.9. If it is not the case, boundary boxes are too 'rough'. In that case, there is a high possibility that the surrounding pipe spools of that equipment also fall into its boundary box. EP2 (equipment 2) in figure 7.9 is such an example. Consequently, the spatial relationship between EP2 and spool 1 will be detected by the model as intersection, but in reality, they may be free from any conflict and can be installed at the same time.

In such a case, the information of the real positions of at least two points of equipment and one size (width, length or height) should be known in order to locate the equipment more precisely in a ship. However, such information is not always available in the digital engineering model or cannot be found in the shipyard database. This may be caused by the involvement of so many subcontractors not all working in the same model or not all using the same work procedures. Under such conditions, manual input had to be adopted. The measurement of the real position of equipment was based on the detailed engineering 3D model.

\section{Connection points of equipment}

A system in a ship consists of equipment and pipes, ducts or other components to fulfill a certain function. For example, lubrication systems are used to provide lubrication to equipment like diesel engines, gas turbines and gearboxes. It contains equipment like the engine, lubricating oil drain tank, coarse filter, cooler, etc. Steel pipes are needed to connect all these pieces of equipment and form a closed system.

In theory, the coordinates of connection points of equipment should be the same as the ending points of pipe spools to which it will be connected. However, in reality, a valve or pump maybe put in between. Or when the joined components are in their final position, the pipe spool is measured on board and then its production sketch is 


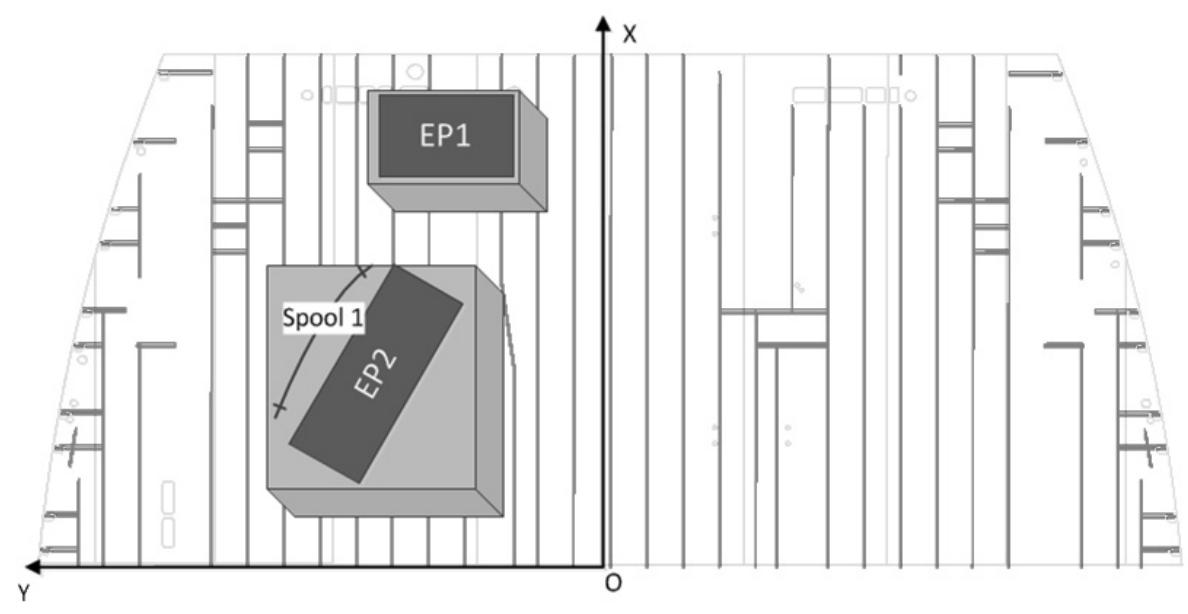

Figure 7.9: The position of equipment boundary box

made. This sketch goes to the pipe shop for prefabrication. Thus, such pipe data may not be available yet during the detailed engineering stage.

Besides, in the XML file, only a batch of spools belonging to the same pipe are known. It is not in general possible to find the starting or ending point of a pipe system among these spools. The method of counting the frequencies of two end points of each piece of component was attempted. If an end point only occurs once, it would have to be a connection point. This turned out to be infeasible, because some pieces were just missing in the data. This leads to points which cannot be the ending points to seem ending points. For these reasons, the information of connection points of equipment is not used in the current version of the sequence generation model. All of this would greatly benefit if automatic routing would be employed, see Asmara (Asmara and Nienhuis, 2008).

\section{Section ID and mounting time}

So far, we have two sources of inputs: the preparation model and the equipment database. The former provides the data relevant to pipes, cable trays and ducts and the latter equipment. Since the section ID is included in the name of a spool, the section ID of equipment serves as an indicator to bring the two inputs together. All types of components, as long as they have the same section ID, will be regarded as outfitting items in that section and participate in the sequence generation model.

The mounting time of equipment should also be known in order to calculate the total duration time of outfitting a section. Unlike the mounting time of pipes, cable trays and ducts which largely depends on their physical attributes, the mounting time of equipment is influenced by too many factors, like the complexity of equipment (pumps, heat exchanger, or switch board), how skillful the workers are, the location 
96 Automatic generation of assembly sequence for the planning of outfitting processes in shipbuilding

of equipment (which strongly influences the accessibility of cranes), the number of connections to be made, the alignment procedures, etc. Consulting with the staff on the worksite, the mounting time of each piece of equipment in the test cases was estimated and input by hand.

In a later stage it will be fairly straight forward to accumulate database with mounting time per piece of equipment. A search algorithm or some form of statistical modeling will then allow automation of this part of the sequence generation model.

In general, due to the fact that we did not take into account the detailed steel structure information which is heavily involved in the detection of equipment's location and because of the complexity of equipment installation, the investigation of equipment assembly sequence is not sufficiently detailed yet. To deal with it thoroughly, further research is still needed and we will discuss it in chapter 9 .

\subsection{Summary}

The main source of data is from the detailed engineering 3D ship model. However, there is no guarantee that this $3 \mathrm{D}$ model itself is able to provide everything needed. Thus, more information is required to provide a proper input set for the sequence generation model. This chapter has detailed the approach to data preparation. Recommendations in relation to further improvements in data collection are:

- The generated XML file obtained by inquiring the 3D model database should be complete without losing bends, spools, pieces of equipment etc.

- Pipe support information should be added in the engineering model, which not only helps to calculate the mounting time of each spool in the research but also shortens the time for determining their position in the steel structures on site.

- Equipment dimension and connection points should be added in the 3D model or at least be traceable.

- The order of coordinates of all pipe spools within one pipe system should be made explicit and arranged in a logical way. This makes it easier to detect which pipe spool is connected to which. This is useful because the spools within one pipe system should preferably be mounted consecutively. In the research, time had to be spent to develop a sensible way to find appropriate spool connections within one system and this often resulted in not ideal and sometimes even wrong results. For example, if pipe spools have branches, it is not possible to detect which spool should be regarded as the main trunk and which one as a branch. Combination with the work of automatic pipe routing will be very helpful in this respect. 
- More data concerning the mounting time of pipes, HVAC and equipment should be collected to improve its accuracy, for instance with the help of regression analysis. 



\section{Chapter 8}

\section{Assembly Sequence Generation Model}

The previous chapter described what kind of data are needed and the way to obtain them. Taking these data as the necessary input, this chapter mainly presents how the assembly sequence generation model works. Section 8.1 focuses on the working principles of the model and the output of the model has also been illustrated here. Section 8.2 explores the possibilities to incorporate more functionality into the sequence generation model. Section 8.3 describes further improvements of the model.

\subsection{Description of the assembly sequence generation model}

The goal of this model is to generate a reasonable assembly sequence of non-structural components in the outfitting processes, under the assumption that the various resources, such as drawings, material, equipment and workers with certain skills, etc. required to perform the activities are always available. The research only takes the geometrical aspects of components into consideration. In chapter 6.1 , the physical attributes of outfitting components that may influence their mounting order were discussed and selected. Based on this, the working principles of the model are explored and developed in order to achieve the goal. This section presents how the model works, taking all these attributes into consideration step by step.

The generation of the assembly sequence comprises of six major steps.

Step 1: Perform the height iteration with the application of the interference matrix, with a purpose of detecting the spatial relationships among outfitting components (including spools and equipment). The definition of 'spool' can be found in chapter 7.1.2. The position ranking derives from this step, see chapter 6.2.1 and chapter 6.3.2;

Step 2: Rank the components based on their material type, weight, size and penetration, see chapter 6.3.2; 
100 Automatic generation of assembly sequence for the planning of outfitting processes in shipbuilding

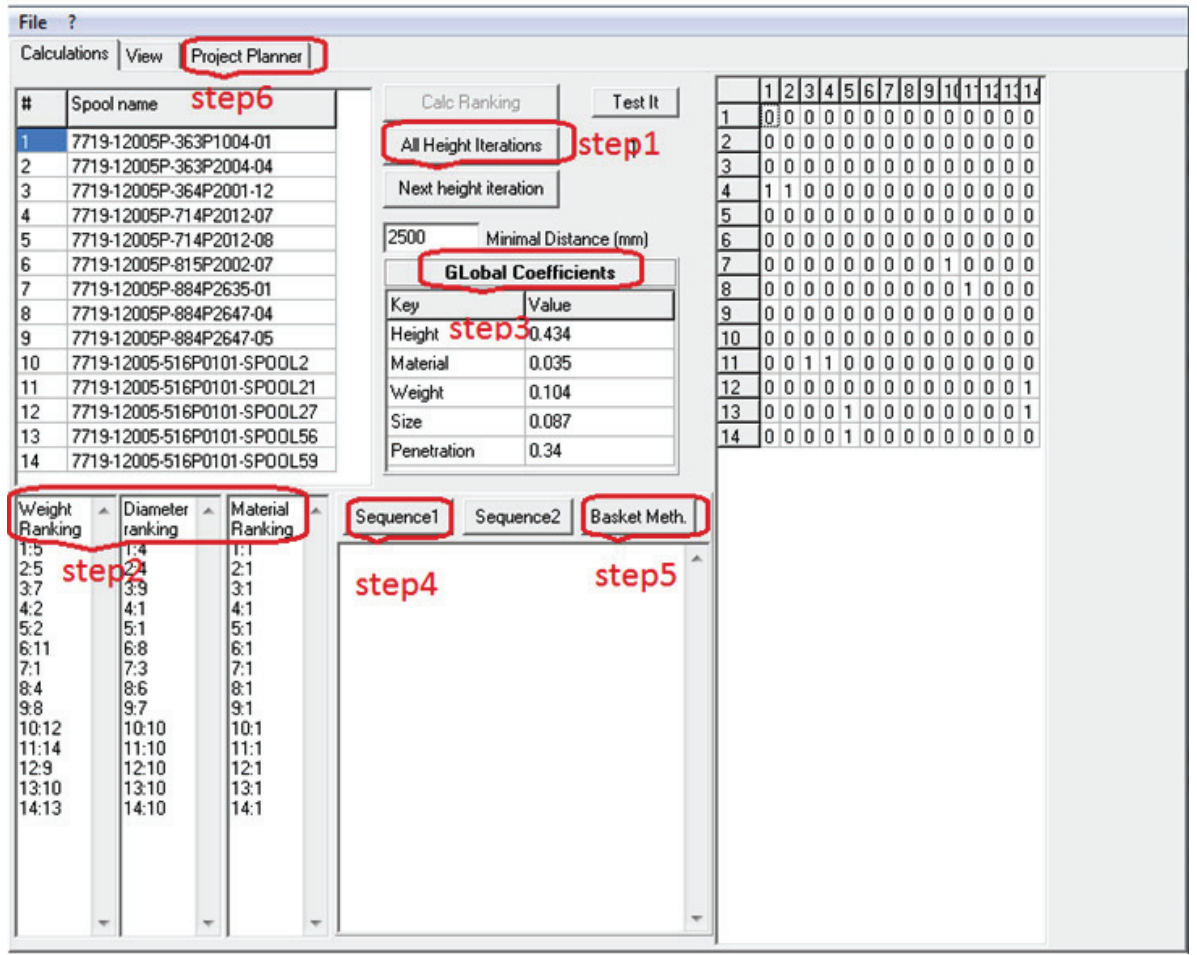

Figure 8.1: 6 steps on the operating screen of the model

Step 3: Use AHP to estimate the relative importance of each physical attribute in deciding the mounting order, see chapter 6.3.1;

Step 4: Obtain the mounting order in a sequential manner, see chapter 6.3.2;

Step 5: Use the 'Basket Done method' which will be explained in detail in this section, taking the spatial accessibility into consideration, to group as many components as possible that can be installed in parallel;

Step 6: Display the assembly sequence by means of the Gantt Chart, which is able to present the finish-start relationships between any component and the rest.

Step 1-4 have been described in the previous chapters. Step 5 and 6 will be specified in the following two subsections. To appreciate the relation between this method and the user interface, each step is visualized in figure 8.1, showing the operating screen of the model.

\subsubsection{Generate assembly sequence in parallel by "Basket Done method"}

In chapter 6.3, the assembly sequence in which components are assembled sequentially, was obtained. On the work site, (several teams of) workers often install several components simultaneously. With a purpose to detect which components can be in- 


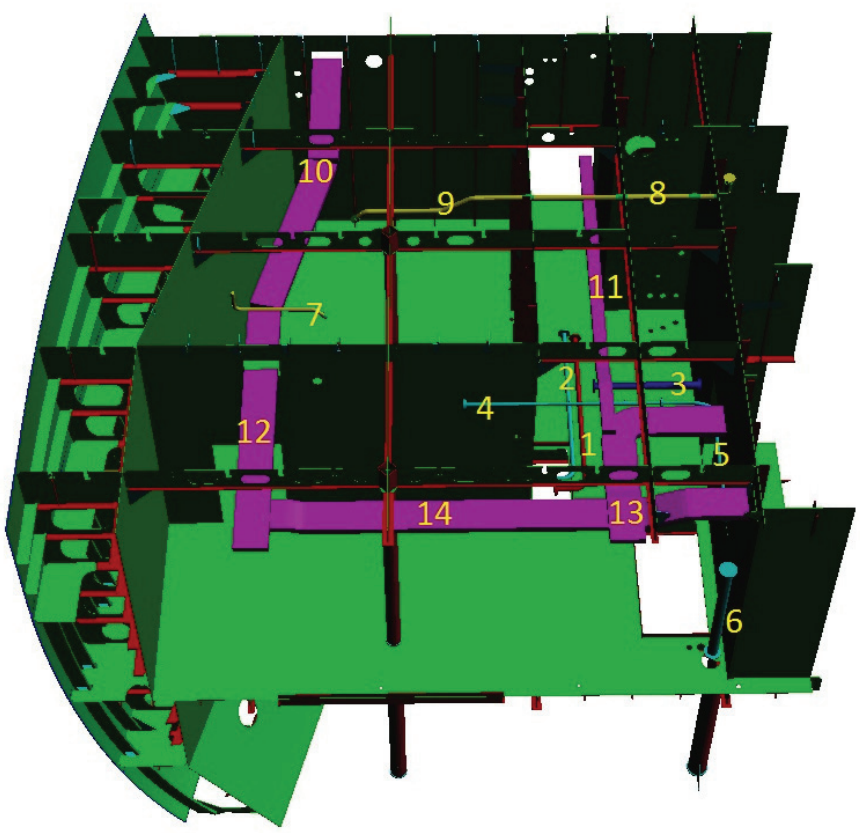

Figure 8.2: A test case with 14 components

stalled in parallel, a new method was developed, called the 'Basket Done Method'. It takes the minimum work distance into consideration. Thus, it contributes to further unveiling the possible finish-start relationships among components. The essence of this method is explained with the help of a 14-component test case seen in figure 10.2. The detailed description has been made in the flowchart in appendix D.

- Step 5.1: Get the result from the sequential method (steps 1-4 above). The result has been sorted by the descending score. The components with higher score will be assembled earlier. The assembly sequence of the 14-component test case is shown in table 8.1. The first column is the ID number of each component, the second column its position ranking (see chapter 6.3) and the last its total score.

- Step 5.2: Group the components according to their position ranking. Within each group, components are listed according to their descending score. Table 8.1 should remain the same because the position ranking of components in the original sequence has also been listed in descending order. 
102 Automatic generation of assembly sequence for the planning of outfitting processes in shipbuilding

Table 8.1: The mounting order in a sequential manner of 14 components obtained from step 1-4

\begin{tabular}{ccc}
\hline Position Ranking & Component ID & Total score \\
\hline 4 & 8 & 12,38 \\
\hline 3 & 11 & 7,66 \\
3 & 13 & 7,55 \\
3 & 12 & 7,45 \\
\hline 2 & 14 & 6,67 \\
2 & 7 & 6,53 \\
2 & 4 & 4,51 \\
\hline 1 & 10 & 4,39 \\
1 & 6 & 3,94 \\
1 & 3 & 3,51 \\
1 & 9 & 3,44 \\
1 & 1 & 2,91 \\
1 & 2 & 2,91 \\
1 & 5 & 2,34 \\
\hline
\end{tabular}

- Step 5.3: Create baskets based on the minimum work distance by using Basket 1 and Basket 2, starting from the group of components which have the highest position ranking.

- Put all components of the group in basket 1;

- Check the minimum distance among the components in basket 1 ; keep the ones satisfying the requirement of minimum work distance in basket 1 and the remainder goes to the second basket.

Taking each component as a line segment, the calculation of the minimum distance between two components amounts to finding the shortest distance between two line segments. The algorithm to calculate the shortest distance, including the Pascal program code, can be found in Hoffmann (Hoffmann, 2004). Figure 8.3 shows the distance between any two components in this test case. Take the seven components which have the position ranking 1 as an example. Taking $2.5 \mathrm{~m}$ as a standard minimum distance, we translate the mutual distances into table 8.2 by setting boolean values. If the distance of two components is equal to or bigger than $2.5 \mathrm{~m}$, the value ' 1 ' is used to indicate the possibility to install them at the same time; otherwise 0 . When we choose the component to compare its distance with other components, we try to keep its original mounting order as much as possible. For example, among these seven components, component 10 is the first one to be mounted, see table 8.1. We 


\begin{tabular}{|l|c|c|c|c|c|c|c|c|c|c|c|c|c|c|c|}
\hline & 1 & 2 & 3 & 4 & 5 & 6 & 7 & 8 & 9 & 10 & 11 & 12 & 13 & 14 \\
\hline 1 & 0 & 160 & 366 & 105 & 1220 & 2656 & 3837 & 2707 & 2777 & 4757 & 390 & 4346 & 535 & 435 \\
\hline 2 & 160 & 0 & 492 & 105 & 1379 & 2839 & 3668 & 2608 & 2648 & 4577 & 544 & 4126 & 691 & 624 \\
\hline 3 & 366 & 492 & 0 & 737 & 708 & 2877 & 4249 & 3489 & 3597 & 5050 & 394 & 5041 & 1080 & 2589 \\
\hline 4 & 105 & 105 & 737 & 0 & 12 & 2667 & 2777 & 4081 & 4075 & 3197 & 215 & 3057 & 371 & 1940 \\
\hline 5 & 1220 & 1379 & 708 & 12 & 0 & 1401 & 5346 & 4095 & 4474 & 6057 & 871 & 5984 & 115 & 186 \\
\hline 6 & 2656 & 2839 & 2877 & 2667 & 1401 & 0 & 7235 & 6439 & 6897 & 7679 & 2784 & 7100 & 2211 & 1576 \\
\hline 7 & 3837 & 3668 & 4249 & 2777 & 5346 & 7235 & 0 & 3924 & 2296 & 161 & 4185 & 1122 & 5022 & 3727 \\
\hline 8 & 2707 & 2608 & 3489 & 4081 & 4095 & 6439 & 3924 & 0 & 0 & 3510 & 240 & 5370 & 4450 & 6023 \\
\hline 9 & 2777 & 2648 & 3597 & 4075 & 4474 & 6897 & 2296 & 0 & 0 & 810 & 1018 & 3661 & 4693 & 6013 \\
\hline 10 & 4757 & 4577 & 5050 & 3197 & 6057 & 7679 & 161 & 3510 & 810 & 0 & 4319 & 260 & 5543 & 2877 \\
\hline 11 & 390 & 544 & 394 & 215 & 871 & 2784 & 4185 & 240 & 1018 & 4319 & 0 & 5140 & 250 & 1271 \\
\hline 12 & 4346 & 4126 & 5041 & 3057 & 5984 & 7100 & 1122 & 5370 & 3661 & 260 & 5140 & 0 & 5290 & 100 \\
\hline 13 & 535 & 691 & 1080 & 371 & 115 & 2211 & 5022 & 4450 & 4693 & 5543 & 250 & 5290 & 0 & 199 \\
\hline 14 & 435 & 624 & 2589 & 1940 & 186 & 1576 & 3727 & 6023 & 6013 & 2877 & 1271 & 100 & 199 & 0 \\
\hline
\end{tabular}

Figure 8.3: Distance calculation between any two components in the 14-component test case

Table 8.2: The distance comparison of seven components

\begin{tabular}{llllllll}
\hline ID & 1 & 2 & 3 & 5 & 6 & 9 & 10 \\
\hline 1 & 0 & 0 & 0 & 0 & 1 & 1 & 1 \\
2 & & 0 & 0 & 0 & 1 & 1 & 1 \\
3 & & & 0 & 0 & 1 & 1 & 1 \\
5 & & & & 0 & 0 & 1 & 1 \\
6 & & & & & 0 & 1 & 1 \\
9 & & & & & & 0 & 0 \\
10 & & & & & & & 0 \\
\hline
\end{tabular}

first check which components could be installed at the same time with component 10. Thus component 10 remains in Basket 1 and does not go to Basket 2. As the next in line, we conclude that component 6 can be installed in parallel. The next one would be component 3 which can indeed be installed in parallel with both component 10 and 6 . Component 9 does not satisfy the distance criterion and is moved to Basket 2. And so on.

After step 5.3, Basket 1 contains component 10, 3 and 6, and Basket 2 now contains component $1,2,5$ and 9 .

- Step 5.4: Look for components with the shortest mounting time in Basket 1. There could be more than one. These components are mounted and subsequently moved to Basket Done, while recording their ID and mounting time.

- Step 5.5: As soon as a component is moved out of Basket 1, look for components in Basket 2 to find which ones fit in with the remaining spools in Basket 1 satisfying the criterion of minimum work distance, starting from the component having the highest total score. Such components are the successors of components in Basket Done and their waiting time should be the mounting 
104 Automatic generation of assembly sequence for the planning of outfitting processes in shipbuilding

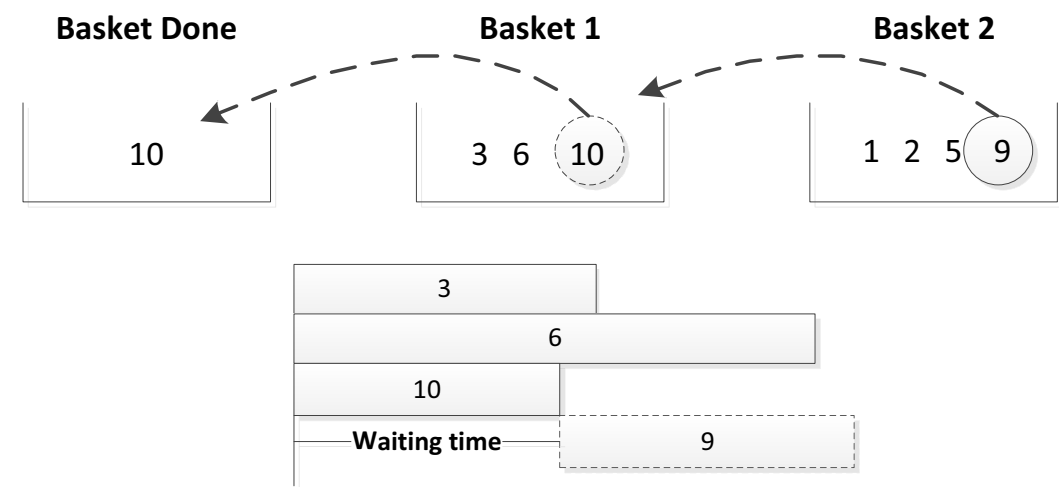

Figure 8.4: Illustration of Basket Done Method

time of spools which just went into Basket Done.

Figure 8.4 further illustrates how steps 5.4 and 5.5 work. Component 10 has the shortest mounting time, compared with the other components in Basket 1 and was sent to Basket Done after its mounting was completed. Component 9 in Basket 2 satisfies the requirement set in step 5.5 and thus, was transferred to Basket 1. Thus, component 10 is the predecessor of component 9 and its mounting time is the waiting time of component 9 . The new mounting time of component 9 becomes the sum of its waiting time and its own mounting time. At the moment component 9 starts, component 3,6 and 9 stay in Basket 1.

- Step 5.6: Repeat step 5.4-5.5, until Basket 2 is empty.

In Basket 1, since component 3 has the shortest mounting time, compared to component 6 and 9, it goes to Basket Done first. Then, component 1 in Basket 2 has been chosen to enter Basket 1. Repeat step 5.4-5.5 and the following component coming out from Basket 2 is component 5 , finally component 2 .

- Step 5.7: Move all remaining components in Basket 1 to Basket Done. There are no finish-start relationships among them. Figure 8.5 shows the assembly sequence of these seven components according to the Basket Done method.

- Step 5.8: When Baskets 1 and 2 are empty, move to the next height level and repeat steps 5.2-5.7 until components in all height levels have been cleared.

Clearly, this method requires the mounting time for assembling each spool. Although the way to calculate the mounting time for pipe and HVAC duct piece is 


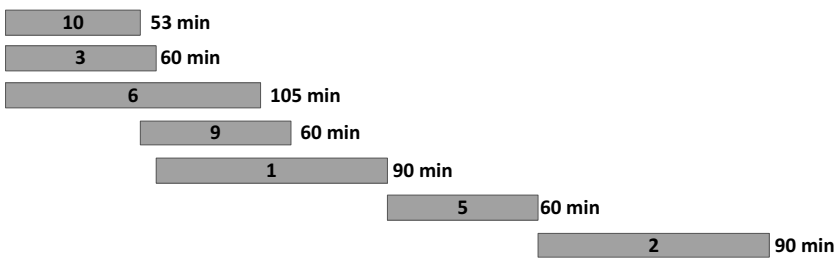

Figure 8.5: The assembly sequence of seven components after the Basket Done method

described in appendix B, it is summarized here.

- Pipe piece: 30 minutes for preparation; 45 minutes for transporting it by crane if the weight of a pipe spool is more than $50 \mathrm{~kg}$ and if the weight is less than $50 \mathrm{~kg}$, there is no such time; 30 minutes for each support including measuring, making and welding it. If the length of a pipe spool is less than $3000 \mathrm{~mm}$, at least one support is needed.

Mounting time $=30+45($ or 0$)+30 *($ length/3000). $3000 \mathrm{~mm}$ is the span between every support.

- HVAC duct: On average, 19 minutes is needed to connect a coupling with straight ducts.

Mounting time $=19^{*} \mathrm{n}, \mathrm{n}$ is the number of couplings

- Cable tray: 10 minutes for making a pair of supports; 5 minutes for aligning the current cable tray with previous or surrounding ones; 2 minutes for welding them on the supports. If the length of a piece of cable tray is less than 3000 $\mathrm{mm}$, at least one pair of supports is needed.

Mounting time $=10 *($ length/3000) $+5+2 *($ length/3000). $3000 \mathrm{~mm}$ is the span of every pair of support.

- Equipment: through consulting the staff on the worksite, the mounting time of each piece of equipment in the test cases was estimated.

\subsubsection{The output of the model}

To achieve the research goals set up in section 1.3, the model should be able to:

- Generate a reasonable mounting sequence for outfitting processes;

- Show the finish-start relationships by displaying the sequence by means of a Gantt Chart 
106 Automatic generation of assembly sequence for the planning of outfitting processes in shipbuilding

Table 8.3: The final assembly sequence of 14-component in text

\begin{tabular}{ccc}
\hline Component ID & Actual mounting (minute) & Predecessors' ID \\
\hline 8 & 60 & \\
12 & 29 & \\
11 & 41 & 11 \\
13 & 29 & \\
14 & 53 & \\
7 & 60 & 14 \\
4 & 60 & \\
10 & 53 & \\
3 & 60 & 10 \\
6 & 105 & 3 \\
9 & 60 & 1 \\
1 & 90 & 5 \\
5 & 90 & \\
2 & 60 & \\
\hline
\end{tabular}

- Output the assembly sequence to a planning software.

The first two goals help planners, both in subcontracted companies and the shipyard itself, to understand the assembly process better by providing them with finish-start relationships of mounting activities. Meanwhile, outputting the assembly sequence into a planning software, for example Microsoft Project or Primavera, enables the planners to access such results. Consequently, they may arrange more reliable production schedules, including the reasonable estimation of pre-outfitting time and the leveled usage of resources. It may make the result of the research more useful in a practical way. This section is devoted to the description of the outputs of the model, which are:

- the display of the assembly sequence in text;

- the display of the assembly sequence in Gantt Chart;

- the visualization of the assembly sequence by $3 \mathrm{D}$ animation.

Here, the 14-component test case mentioned in the previous section is used to illustrate how the model displays its assembly sequence in text and in Gantt Chart, and visualizes the assembly sequence in a $3 \mathrm{D}$ animation. In text, the result of the test case is shown in table 8.3. If a particular spool does not have any predecessors, the entry under 'Predecessors' is left empty.

If the text file is opened by Excel, it could be easily accessed by other planning softwares, like Microsoft Project, Mars Planning or Primavera. This makes it possible 


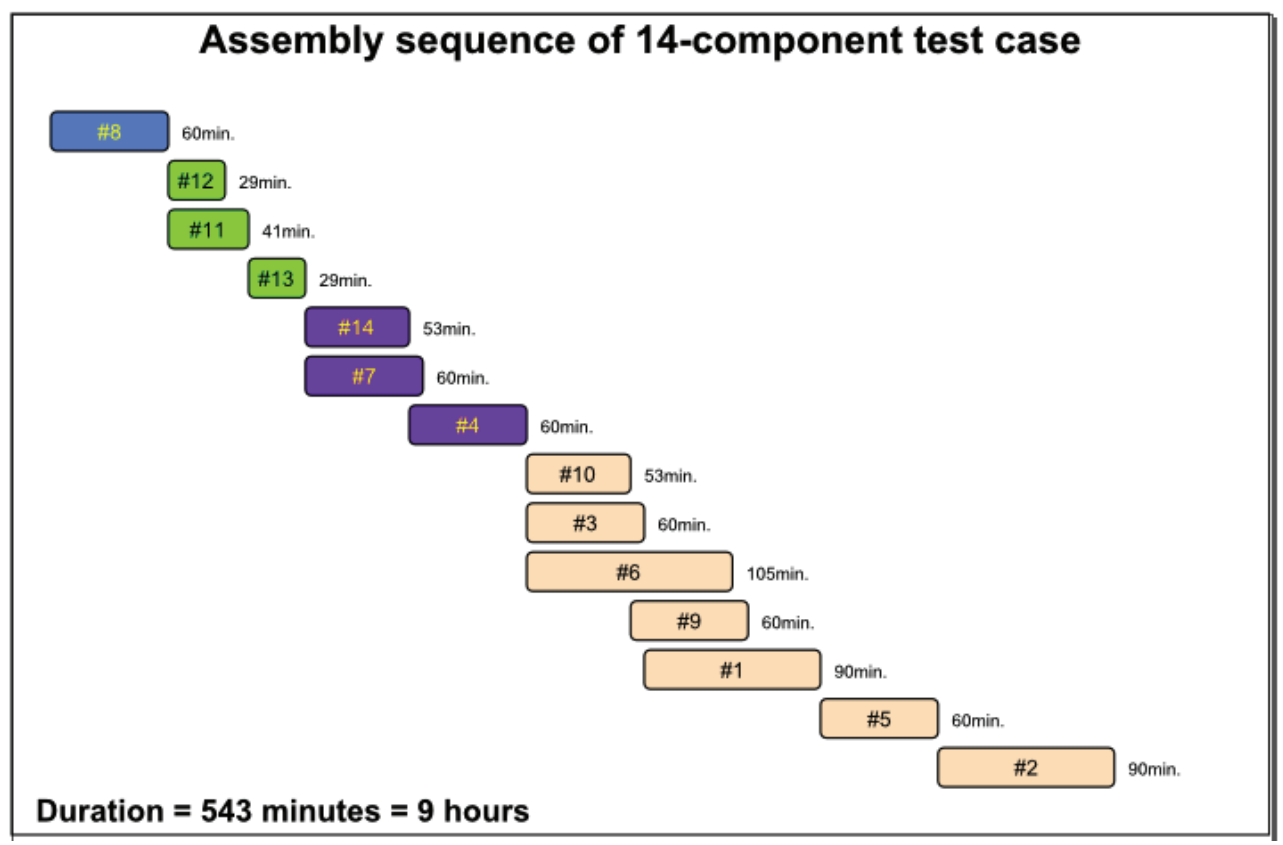

Figure 8.6: The final assembly sequence of 14-component in Gantt Chart

for planners to use the result of the model directly once the layout of the excel file is (automatically) modified according to the input requirement for the applied planning software.

Visualizing the sequence can be done by a Gantt Chart that directly shows durations and start-finish relationships. A Gantt Chart is a bar chart that illustrates a project planning, putting activities as separate bars on a time line. The display of the above result by a Gantt Chart can be seen in figure 8.6. Each bar represents the assembly of a component. The ID of a component is in the middle of the bar and the mounting time at the right side of the bar. The bars with the same color mean that components represented by them have the same height level.

Besides, the assembly sequence can also be animated in a 3D view, which provides planners with a better appreciation of the order in which components are mounted in the assembly sequence. The 3D view example of the 14-component case is presented in figure 8.7, in which the snapshots after 4, 8, 11 and 14 components are installed were displayed. Consecutively clicking the 'Next' or 'Previous' button on the operation screen allows the generation of a simple animation. 
108 Automatic generation of assembly sequence for the planning of outfitting processes in shipbuilding

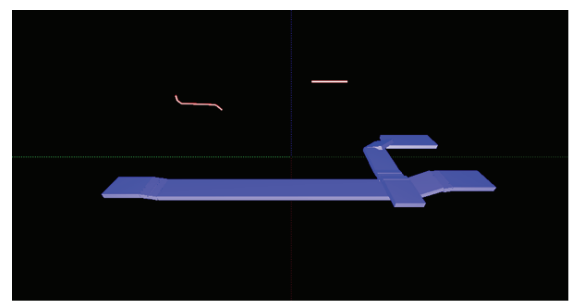

after 4 components

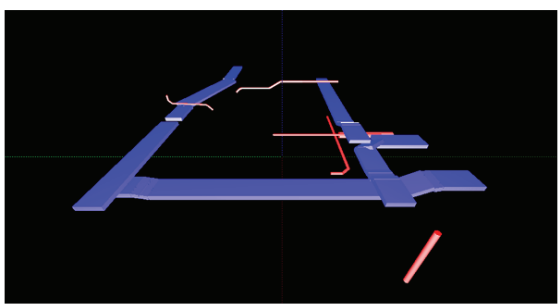

after 11 components

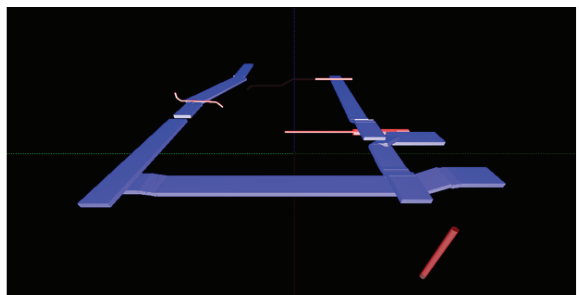

after 8 components

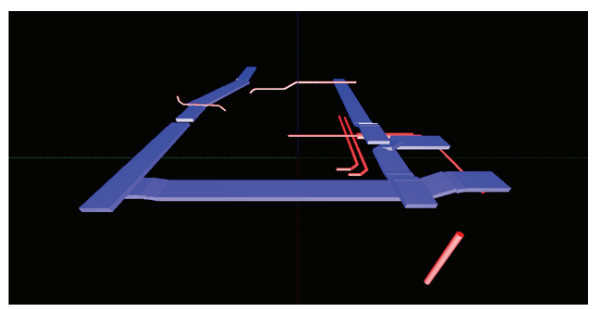

after 14 components (finished)

Figure 8.7: The final assembly sequence of the 14-component case in 3D animation

\subsection{Added functionality}

In this section, we explore the possibilities to incorporate more functionality into the sequence generation model. These functions are resource leveling, handling component delays and generating the assembly sequence of components according to their categories.

\subsubsection{Resource leveling}

As mentioned in chapter 1.3, the research focuses on the technical aspects when dealing with the assembly sequence. It pays little attention to the resources that are indispensable to convert empty steel structures into sections and compartments that are fully equipped with outfitting components. The assembly sequences possible according to the presented method may lead to an unreasonable resource allocation or may surpass the resource capacity and thus, may result in low productivity and/or high production costs.

In outfitting processes, these resources primarily are workers, cranes and transportation vehicles. Largely, cranes and transportation vehicles in shipyards are also heavily involved in the steel structure construction process. Thus, it is difficult to trace and assign their usage for outfitting purposes. Indeed, their unavailability when needed during outfitting stages causes longer waiting times. This has already being taken into account when estimating the mounting time of assembling outfitting components. For workers, their allocation is directly influenced by the possible as- 
sembly sequences. Therefore, this section mainly discusses the allocation of human resources in outfitting processes.

On the work site, usually a team consisting of two workers carries out the assembly work. For example, to assemble a pipe spool, one worker is busy with measuring its position on the steel structure by reading the drawings and the other may make the supports for it in the warehouse nearby. Based on this fact, figure 8.8 shows the human resource distribution according to the final assembly sequence of the 14component case. Apparently, by following the generated assembly sequence of this test case, the number of workers fluctuates greatly throughout the whole assembly process.

Even if several sections are outfitted at the same time, to transfer work teams frequently from one section to another may still slow down the work progress. This is because when workers are assigned to work in a new section, they need more time to read the new drawings, get familiar with the environment (like where the nearest power supply is, where the entrance of scaffoldings is, etc.), figure out what previous components were mounted and what is next to be mounted, and prepare their tools before they start the real assembly work. Therefore, technically the feasible assembly sequences may not be realistic from a human resource management aspect.

In order to minimize the variations in human resource demand, some resource leveling was done with the help of Microsoft Project while not violating the finishstart relationships obtained from the assembly sequence. In this 14-component test case, we suppose there are either two, four or six workers available for the outfitting work, with two as a work team. Workers were manually assigned to certain assembly tasks. After resource leveling by Microsoft Project, figure 8.9 shows how the minimum duration is influenced by the number of work teams. The human resource distributions with two, four and six workers can be found in figure 8.10. Obviously, the trend is that the more work teams there are, the shorter the minimum duration. Practically, the number of work teams is however limited by the work space. This explains the less than proportional reduction in duration when increasing the number of workers 4 to 6 .

As the number of components to be installed increases, it is no longer possible to accommodate the assembly sequence with an unlimited number of workers because in some situations, more than ten components can be assembled simultaneously which would require as many teams. When performing the resource leveling, manually assigning workers to hundreds of assembly tasks while not exceeding the maximum allowed outfitting time according to the production plan is not feasible. By coupling this method with a suitable resource leveling algorithm, this can be routinely performed. Thus, to combine the resource limitation and resource leveling with the feasible assembly sequence requires some further work. 
110 Automatic generation of assembly sequence for the planning of outfitting processes in shipbuilding

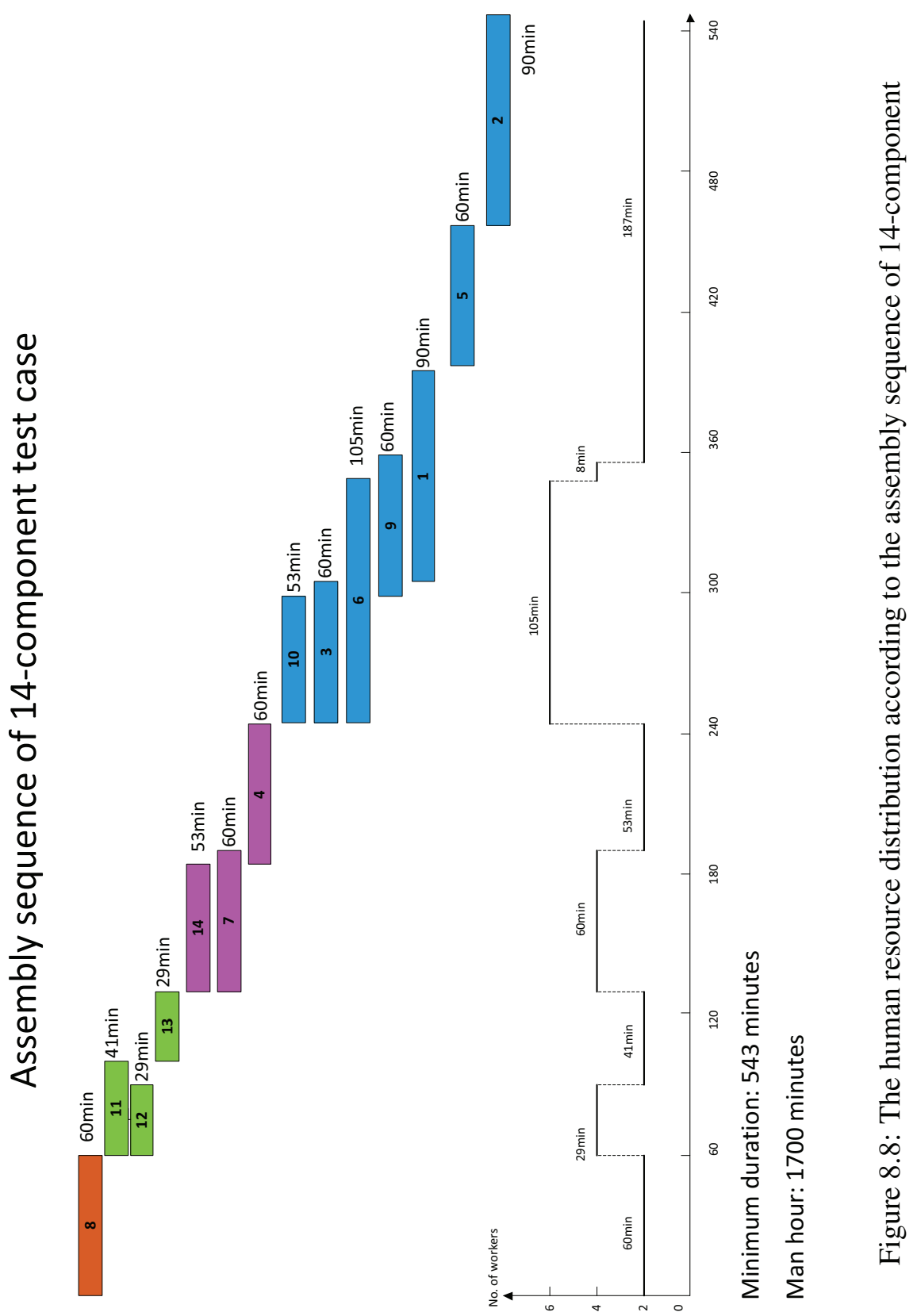




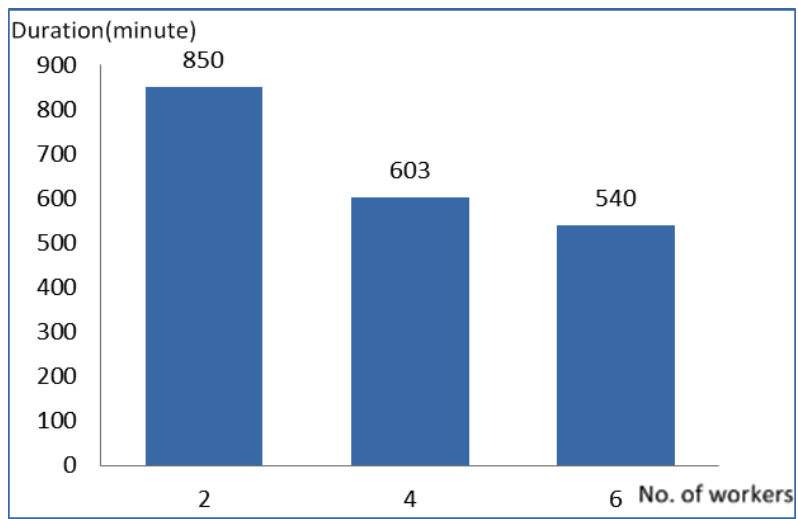

Figure 8.9: The minimum duration of the 14-component case influenced by the number of workers

\subsubsection{Component delay}

Component delay often causes domino effect. The delay of a single component may require postponing the assembly of other components, because the other components block its way or the other components are connected to it. First, we look at why a component could be delayed. Then, the possibility to handle the delay by the current model is discussed.

According to a senior foreman of a piping team, on average less than approximately $5 \%$ of pipe spools in a whole ship are delayed. This statement is based on the record of a piping company plus the foreman's experience. The reasons for any delay could be for example:

- In the engineering stage, pipe routing comes too late;

- Modifications of engineering information may take place in the late stage so that pipe sketches are not ready in time for production;

- Pipes with special flanges and dimensions need more time for fabrication;

- Galvanized and painted pipes take more time and the fabricating shops are too busy to fabricate them on time;

- A component is damaged during transport and needs to be repaired or produced again.

Regarding cable trays, the electrical supervisor described that it is extremely rare that a cable tray could be delayed. If it is delayed, the main reason is a late modification of engineering information. Even if the delay happens, usually it concerns only small pieces of cable trays and it is not a problem to put them in at the late stage. For 
112 Automatic generation of assembly sequence for the planning of outfitting processes in shipbuilding
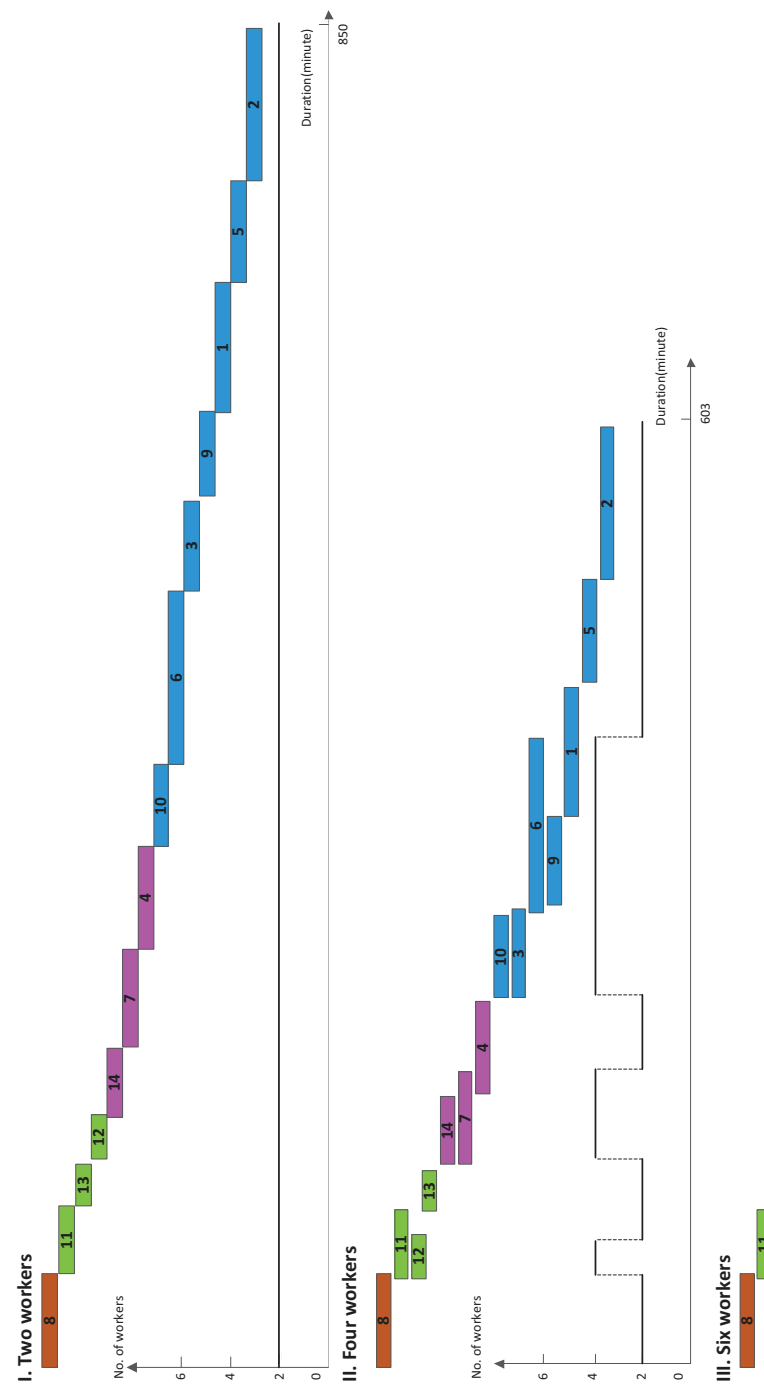

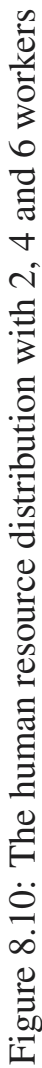


HVAC ducts, at least one HVAC project coordinator was of the opinion it is almost impossible for ducts to be delayed. His statement is also supported by the fact that ducts are standard products. As long as they are purchased in time, their chances of being delayed by fabrication procedures are small. Therefore, any delays mainly happen to pipe spools and equipment.

To deal with the delay, the following factors need to be considered:

- The critical components.

As long as the lead time of a delayed component is not on the critical path of the pre-outfitting/outfitting plan, its delay is not important.

- The milestones of the project.

When a critical component is delayed during the pre-outfitting stage, in reality there are two approaches to solve it. The first way is to extend the pre-outfitting time of the section where this component is located. It requires postponing the hull erection date of that section but such delay should not influence the final ship launching date. This approach is often rejected out of hand by the shipyard even though there are cases where it may be advisable. The second approach is to stick to the hull erection plan and install this component on the slipway. It may cause higher cost because of bad accessibility and more difficult working conditions. Both approaches require the model to be able to distinguish preoutfitting from outfitting.

After initial trials, we must conclude that the current sequence generation model is unable to handle delays in general for the following reasons:

- In the beginning of the research, the assumptions were that material and workers are always available. Based on these assumptions, when developing the method to generate the assembly sequence, we aimed for the best assembly sequence. Alternatives to handle the delay have not been included.

- The current input data is intentionally limited. Analysis of the delay requires the determination of critical components and the incorporation of planning milestones as a constraint. These inputs are lacking at present.

In the future, if the steel structure data is also used, the interference detection should be possible in the $\mathrm{X}, \mathrm{Y}$ and $\mathrm{Z}$ directions. When the component delay happens, alternatives may exist to assemble a component in more than one (X, $\mathrm{Y}$ or $\mathrm{Z}$ ) direction. Indeed, in general one would wish for a much more elaborate interference method. In that case detailed 3D geometries are used to access the possibilities to maneuver a component in the available free space. While certainly possible, it is very questionable if such an approach would yield very superior results. All the uncertainties in the human factors can well be much more important. 
114 Automatic generation of assembly sequence for the planning of outfitting processes in shipbuilding

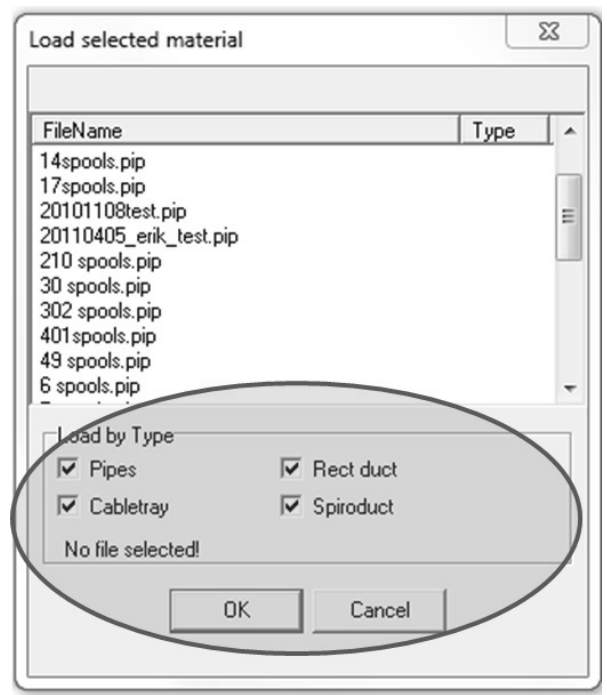

Figure 8.11: Sequence determination of components according to their categories

\subsubsection{Sequence generation by category}

Sequence generation by category means obtaining the assembly sequence only for a certain category (pipe/cable tray/HVAC/equipment). While it is unrealistic to install all equipment together during the pre-outfitting stage, the category approach provides insight into what happens to the sequence and duration if different disciplines work sequentially. In reality, it is possible to do the piping, cable tray or HVAC work category by category although this may lead to the interference problems in the later category.

The current model is able to generate the sequence category by category by loading different types of components as input data. Figure 8.11 is the screen snapshot showing how the model loads different categories of components. This function allows users to focus on a certain category of components. Note however that the method does not check in that case the position constraints caused by the mounting of a previous completed category. This would require a much more cumbersome full geometric analysis of maneuvering a component in the (remaining) free space.

\subsection{Summary}

This chapter mainly presents how the assembly sequence generation model works. All physical attributes of a component and their relative importance in deciding its mounting order were measured and modeled, which resulted in the derivation of finish-start relationships between components. For further implementation of this modeling method, it seems advisable to carry out the following improvements: 
- Develop a way to incorporate the 'mounting stage' factor into the model.

Some components can only be installed when the steel section is open, for example pipes located in the double bottom section, because once the section is closed, it is almost impossible to get these spools into the steel structure and assemble them. Another kind of spools, which cross more than one section, can only be installed after these sections are joined together on the slipway. The installation of equipment is also affected by such a situation. It is very well possible to install small pumps during the pre-outfitting stage. Therefore, outfitting components need to be categorized based on the good accessibility to their surrounding steel structures.

An attempt was already made by assigning 'mounting stage' attributes to each component based on its interaction with the steel structure. This 'mounting stage' is detailed as stages of Pre-outfitting, Slipway Top Open, Slipway Top Closed, Quay and Arbitrary. How to make such an important distinction automatically by a model requires further research. With certainty, the information of the steel structure is indispensable for this.

When a mounting stage factor is included, it can be filtered for component availability. This could in principle be derived from a sufficiently detailed procurement schedule.

- More accurately estimate the mounting time of assembly components.

Because information about pipe, HVAC and cable tray supports is lacking in the engineering model, their number and mounting times were obtained based on rules derived from field observations. In reality, the manufacture time of pipe supports in the warehouse varies, depending on their type and position. Thus, more relevant information needs to be collected in real-life projects.

A videotape on the jobsite is suggested to obtain a record of actual assembly times. It is especially important to automate the calculation of mounting time for pieces of equipment. A detailed questionnaire and subsequent statistical analysis would provide a feasible approach.

- Generate a more reasonable sequence with the consideration of leveled human resources.

The current sequence is optimal from the point of view of minimum duration but it is not automatically acceptable from the point of view of resource management. The current method allows either components to be installed one by one or as many as possible at the same time. A possible approximation is to limit the number of components in Basket 1 when applying the Basket Done Method.

Suppose at the maximum, $\mathrm{N}$ components can be installed at the same time. This corresponds to limiting the maximum number of work teams to N. Af- 
116 Automatic generation of assembly sequence for the planning of outfitting processes in shipbuilding

ter categorizing components into Basket 1 and Basket 2 based on the minimum work distance, only $\mathrm{N}$ components would be allowed to stay in Basket 1 (because components in Basket 1 can be installed in parallel) and the rest still goes to Basket 2. The selection of $\mathrm{N}$ components is based on the descending order of their total scores. Each time if $\mathrm{m}(\mathrm{m} \leq \mathrm{N})$ components are transferred to Basket Done from Basket 1, select $\mathrm{N}-\mathrm{m}$ components from Basket 2 and put them into Basket 1.

However, this method does not guarantee that the $\mathrm{N}$ work teams are occupied as much as possible. It merely shows the peak from the work load, but does not remove the troughs. Possibly, this needs to be combined with a less strict application of the work distance criterion, imposing a penalty on mounting times if two components violate the distance criterion. 


\section{Chapter 9}

\section{Model Application and Evaluation}

Verification is the process of determining that a computational model accurately represents the underlying mathematical model and its solution (ASME, 2006). Validation is the process of determining the degree to which a model is an accurate representation of the real world from the perspective of the intended uses (ASME, 2006). It is sometimes said that verification can be expressed by the query "Are you building it right?" and validation by "Are you building the right thing?" (Boehm, 1981). Both usually are parts of the total model development process. This chapter first describes how the verification of mounting times for different types of components was carried out. Section 9.2 is about the selection of two versions of assembly sequence, Upward version and Downward version. Section 9.3 applies three more cases to test the model. The validation of the assembly sequence and the discussion of duration have also been addressed in this section. The limitations and possible improvements of the model are described in section 9.4. Section 9.5 concludes this chapter.

\subsection{Mounting time verification}

This section describes the way to verify the mounting time. Further interviews with workers, specialized in different disciplines, such as pipe fitting and electrical installation, were conducted. Altogether, 6 senior people, 3 from subcontracted companies and 3 from shipyards, joined in the verification tests. The background of the questionnaire people can be found in appendix E.

The mounting time of assembling each component was calculated based on observations for different types of components. The way to calculate them has been summarized in chapter 8.1.1. Questionnaires, devoted to the verification of mounting time for pipes, cable trays and HVAC ducts were designed. In this section, we illustrate the verification processes for each type of component. The mounting time of each piece equipment was separately estimated through consulting workers on the site. It will not be verified in the research. 
118 Automatic generation of assembly sequence for the planning of outfitting processes in shipbuilding

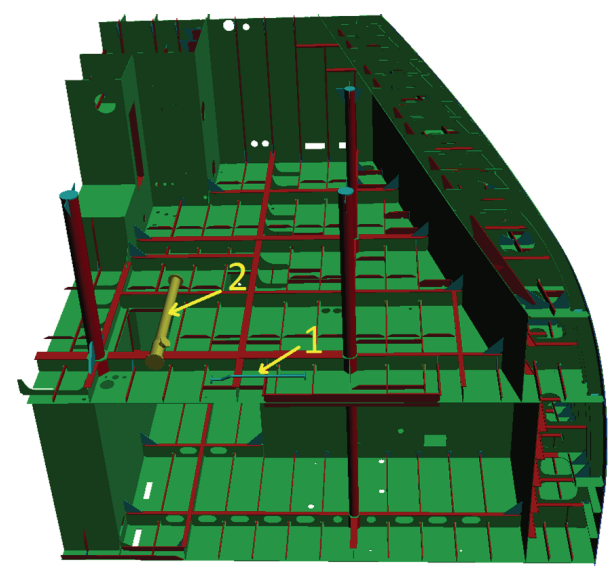

Figure 9.1: Two pipe spools in a section

\subsubsection{The mounting time of pipe spools}

In the verification, two pipe spools were randomly chosen in the section, see figure 9.1. Their geometrical attributes can be found in table 9.1. During the interview, 6 people were asked to estimate the mounting time and the number of supports of these two pipe spools, based on their position in the steel structure and geometrical data. Compared to the results from the model, their answers to the mounting time are shown in figure 9.2. The answers to the number of supports are presented in table 9.2 .

Table 9.1: Geometrical attributes of two pipe spools

\begin{tabular}{ccccc}
\hline Name & Weight $(\mathrm{kg})$ & Length $(\mathrm{mm})$ & Material & Diameter $(\mathrm{mm})$ \\
\hline Pipe spool 1 & 13 & 1920 & ERW 320 & 50 \\
Pipe spool 2 & 132 & 3098 & ERW 320 & 141 \\
\hline
\end{tabular}

Table 9.2: Answers from the questionnaires: estimation of the number of supports of pipe spool 1 and 2

\begin{tabular}{lllllllc}
\hline Item & Q1 & Q2 & Q3 & Q4 & Q5 & Q6 & calculated by model \\
\hline No. of supports for pipe spool 1 & 1 & 1 & 1 & 2 & 2 & 1 & 1 \\
No. of supports for pipe spool2 & 2 & 2 & 3 & 2 & 3 & 2 & 2 \\
\hline
\end{tabular}

Brosens has investigated the mounting time of mounting pipes in his (confidential) master thesis (Brosens, 2008). He took the mounted pipe spools on a particular yard number as an example. He concluded that 1004 pipe spools in the pre-outfitting 


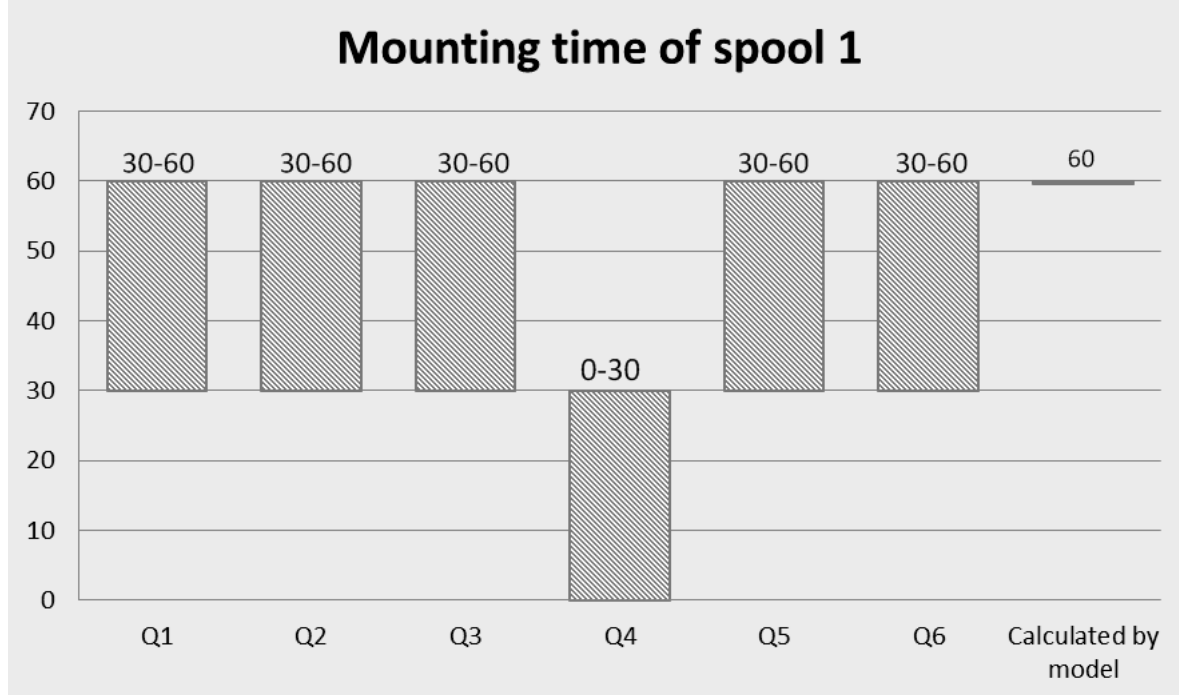

Mounting time of spool 2

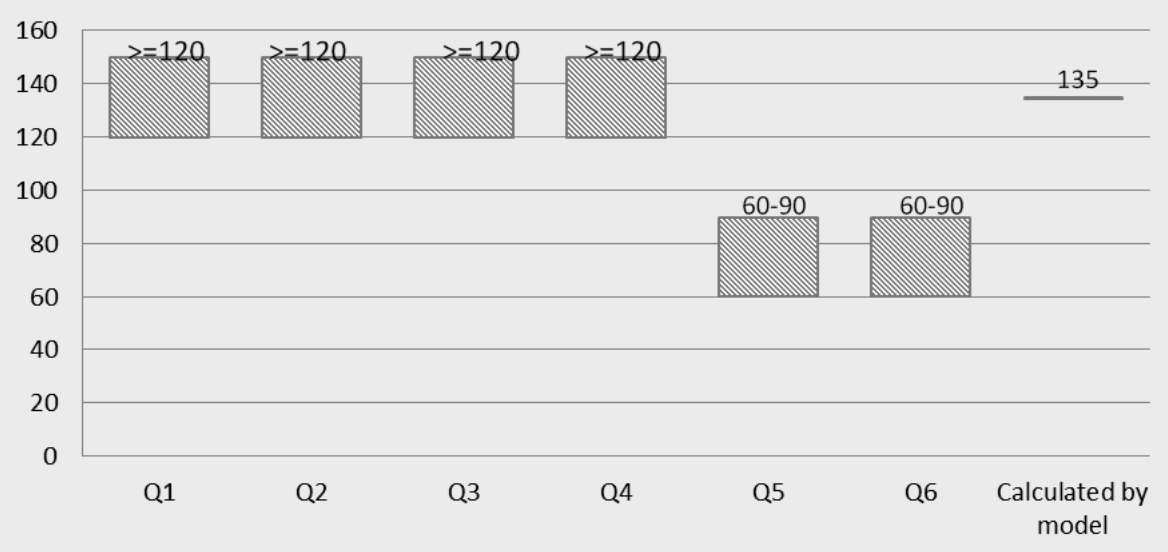

Figure 9.2: Answers from the questionnaires: estimation of mounting time of spool 1 and 2 
120 Automatic generation of assembly sequence for the planning of outfitting processes in shipbuilding

stage need 2899 man-hours and 755 pipe spools in the outfitting stage need 5121 man-hours. Because two pipe fitters are used for the mounting of a pipe spool, the average mounting time of a pipe spool could be obtained as follows:

- Pre-outfitting: 2899 man-hours/ 1004 spools/ 2 persons= 1.45 hours/ spool

- Outfitting: 5121 man-hours/ 755 spools/ 2 persons= 3.4 hours/ spool

We also calculated the mounting time of all pipe spools in the section of an engine room work shop (see chapter 9.2.1). According to the model, 393 spools need 448 hours. The average mounting time of a pipe spool is: 448 hours $/ 393$ spools $=1.14$ hours/ spool.

When comparing the mounting time of pipe spools determined by Brosens with the model, the calculated mounting time is somewhat too low. But there seems to be a fair agreement between the model and the questioned experts. It is because the number of supports is used in the model as a measure to decide the mounting time. This makes the estimation closer to the reality compared to Brosens' conclusion derived from the recorded man-hours which include workers' idle and rest time.

\subsubsection{The mounting time of HVAC ducts}

The mounting time of HVAC ducts, according to the observation, is mainly decided by the number of couplings that ducts have. For the connection of each coupling, 19 minutes are needed. To validate the mounting time, two pieces of ducts in figure 9.3 were presented to the people participating in the questionnaire. Table 9.3 describes their geometrical attributes. Their mounting times are compared in figure 9.4.

In general, the model overestimates the mounting time of ducts. In figure 9.4, the mounting time of the two ducts resulting from the model (152 minutes for duct 1 and 133 minutes for duct 2) is longer than the average results given by the questionnaires (100 minutes for duct 1 and 90 minutes for duct 2). The reason could be that the time for connecting each coupling should be less than 19 minutes.

It is worthwhile to note that the estimates of the worker vary considerably. Obtaining more precise model parameter from such questionnaires will thus be difficult even when allowing for larger test populations.

Table 9.3: Geometrical attributes of two ducts

\begin{tabular}{ccccc}
\hline Name & Weight $(\mathrm{kg})$ & Length $(\mathrm{mm})$ & Material & Diameter $(\mathrm{mm})$ \\
\hline Duct 1 & 38 & 14038 & Sheet metal & 192 \\
Duct 2 & 15 & 13670 & Sheet metal & 112 \\
\hline
\end{tabular}




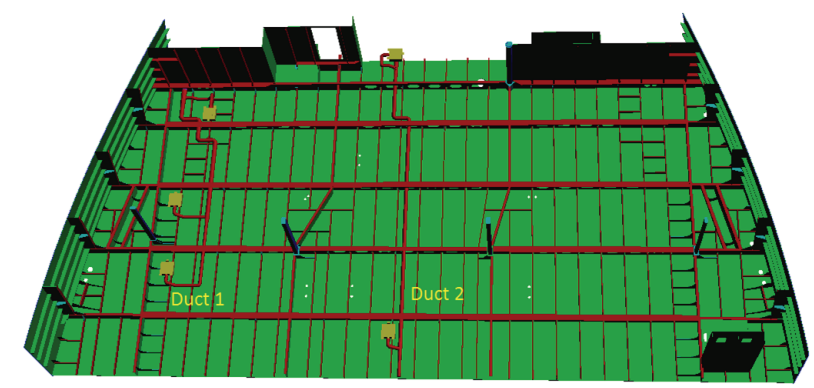

Figure 9.3: Two HVAC ducts in a section

Table 9.4: Geometrical attributes of two cable trays

\begin{tabular}{ccccc}
\hline Name & Weight $(\mathrm{kg})$ & Length $(\mathrm{mm})$ & Material & WxH $(\mathrm{mm})$ \\
\hline Cable tray 1 & 92 & 6856 & Steel 33 & $500 \times 100$ \\
Cable tray 2 & 78 & 5994 & Steel 33 & 200x100 \\
\hline
\end{tabular}

\subsubsection{The mounting time of cable trays}

Apart from the 5 minutes for the alignment of cable trays, their mounting time depends on the number of supports they need. To validate the mounting time, two pieces of cable trays were randomly chosen as shown in figure 9.5. Their corresponding geometrical attributes can be found in table 9.4. The number of supports from both the questionnaires and the model are presented in table 9.5. Their mounting times estimated by six people are compared with the model calculation, as shown in figure 9.6.

Table 9.5 shows that the number of supports for cable tray 2 (3 supports) calculated by the model is rather close to the average number ( 3.3 supports) given by 6 people. Figure 9.6 also shows that the mounting time estimated by the model is 41 minutes, compared to average mounting time from the questionnaires, 46 minutes. For cable tray 1, the value of the model are 4 supports and 53 minutes duration. And the questionnaires show 3.5 supports and 53 minutes duration. All in all, this comparison is quite good.

Table 9.5: Answers from the questionnaires: estimation of the number of supports of cable tray 1 and 2

\begin{tabular}{lllllllc}
\hline Item & Q1 & Q2 & Q3 & Q4 & Q5 & Q6 & calculated by model \\
\hline No. of supports for cable tray1 & 4 & 3 & 3 & 3 & 4 & 4 & 4 \\
No. of supports for cable tray2 & 3 & 3 & 3 & 3 & 4 & 4 & 3 \\
\hline
\end{tabular}


122 Automatic generation of assembly sequence for the planning of outfitting processes in shipbuilding

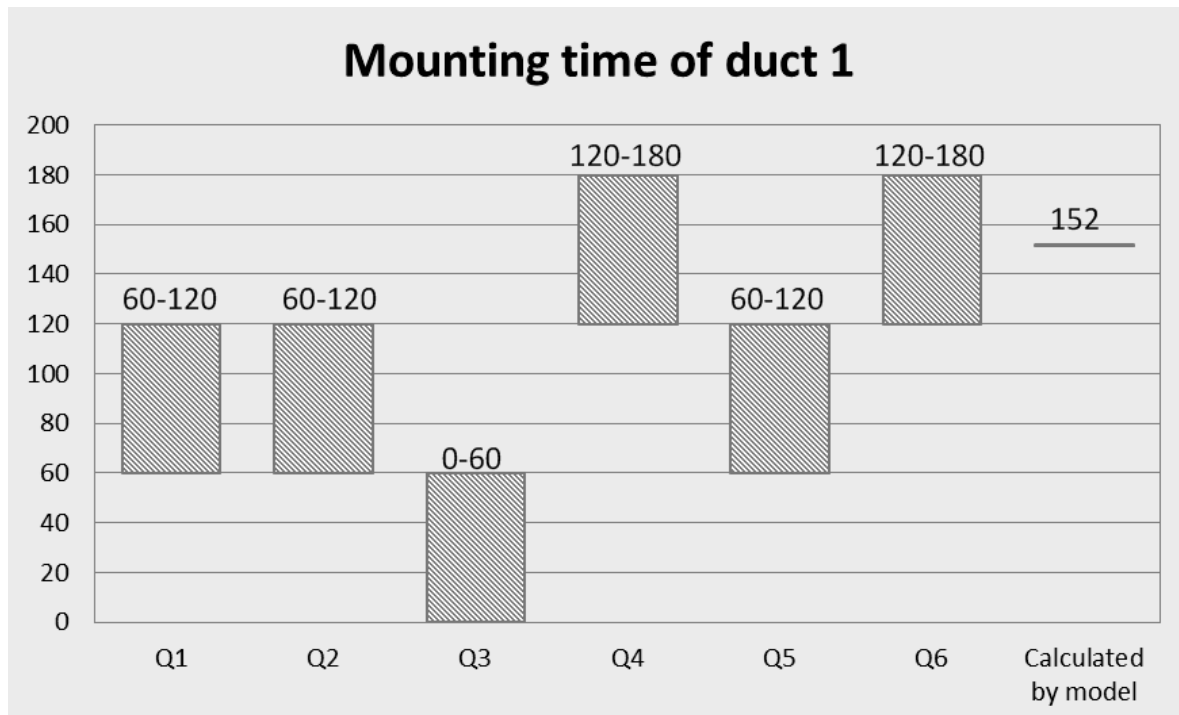

Mounting time of duct 2

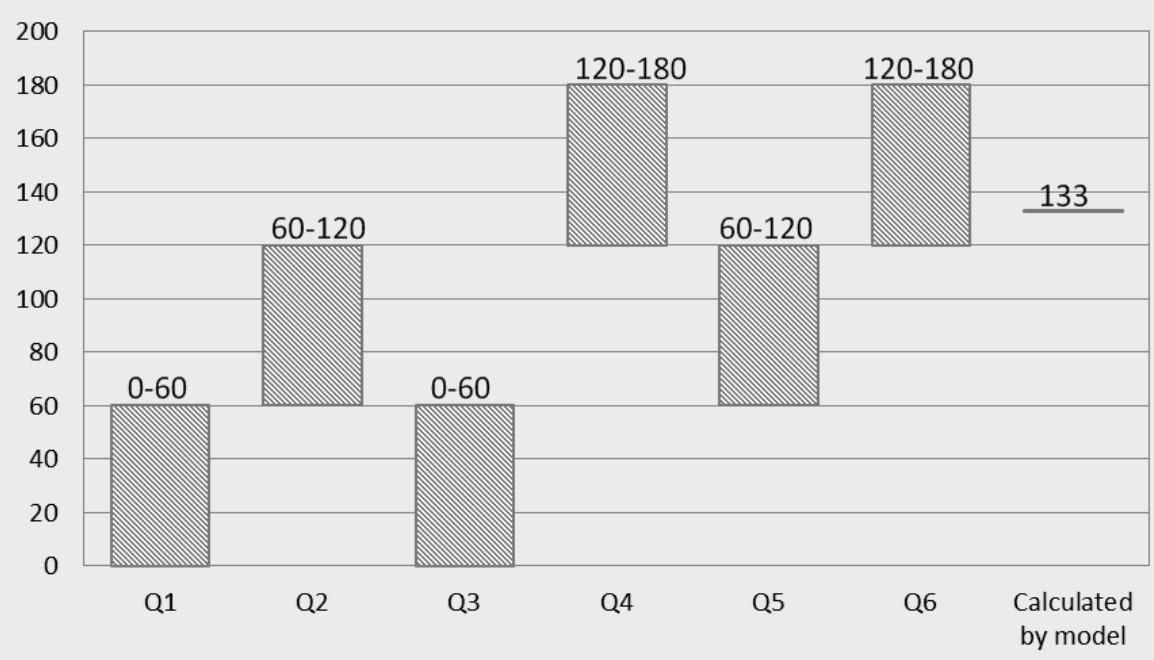

Figure 9.4: Answers from the questionnaires: estimation of mounting time of duct 1 and 2 


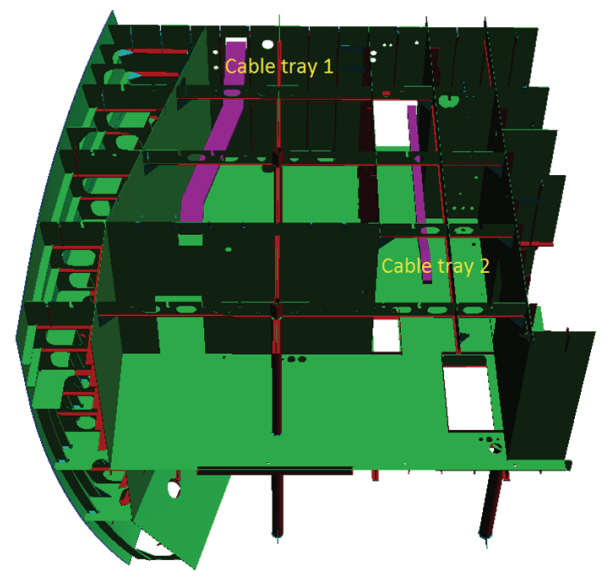

Figure 9.5: Two cable trays in a section

Apart from the mounting time of pipes which could be found in the literature, hardly any other literature investigates the mounting time of HVAC ducts and cable trays. The test cases with two components and six people giving a sample of 24 data points are insufficient to analyze the result statistically. There is therefore no guarantee that the way to calculate the mounting time is reliable. The suitability for further duration calculations should be considered cautiously. Nevertheless, the current assumptions provide insights to calculate the mounting time which is an indispensible index in any kind of production plan. Improvement is possible by non-stop recording of the assembly processes with videos for a certain time and analyzing the factors to determine the mounting time more reliably.

\subsection{Assembly sequences selection}

As mentioned in chapter 6.2.1, when generating assembly sequences, the model was able to give both the 'Downward' version and the 'Upward' version. This part focuses on how to choose the better assembly sequence for a certain assembly scenario.

Two versions of the assembly sequence for different assembly scenarios were presented in the form of questionnaires. The six senior people who joined the mounting time verification tests also participated in the assembly sequence selection. Although the six workers are statistically insufficient to formally validate the research results, it was practically impossible and unacceptable to ask a large number of workers to do the selection. Thus, the result from the questionnaires provides some indications, but by no means a formal validation.

A test case with 10 components (figure 9.7) was used to describe the two versions of the assembly sequence. The results are shown in figure 9.8. Apart from differences in the duration, the main difference between these two versions is that either more 
124 Automatic generation of assembly sequence for the planning of outfitting processes in shipbuilding
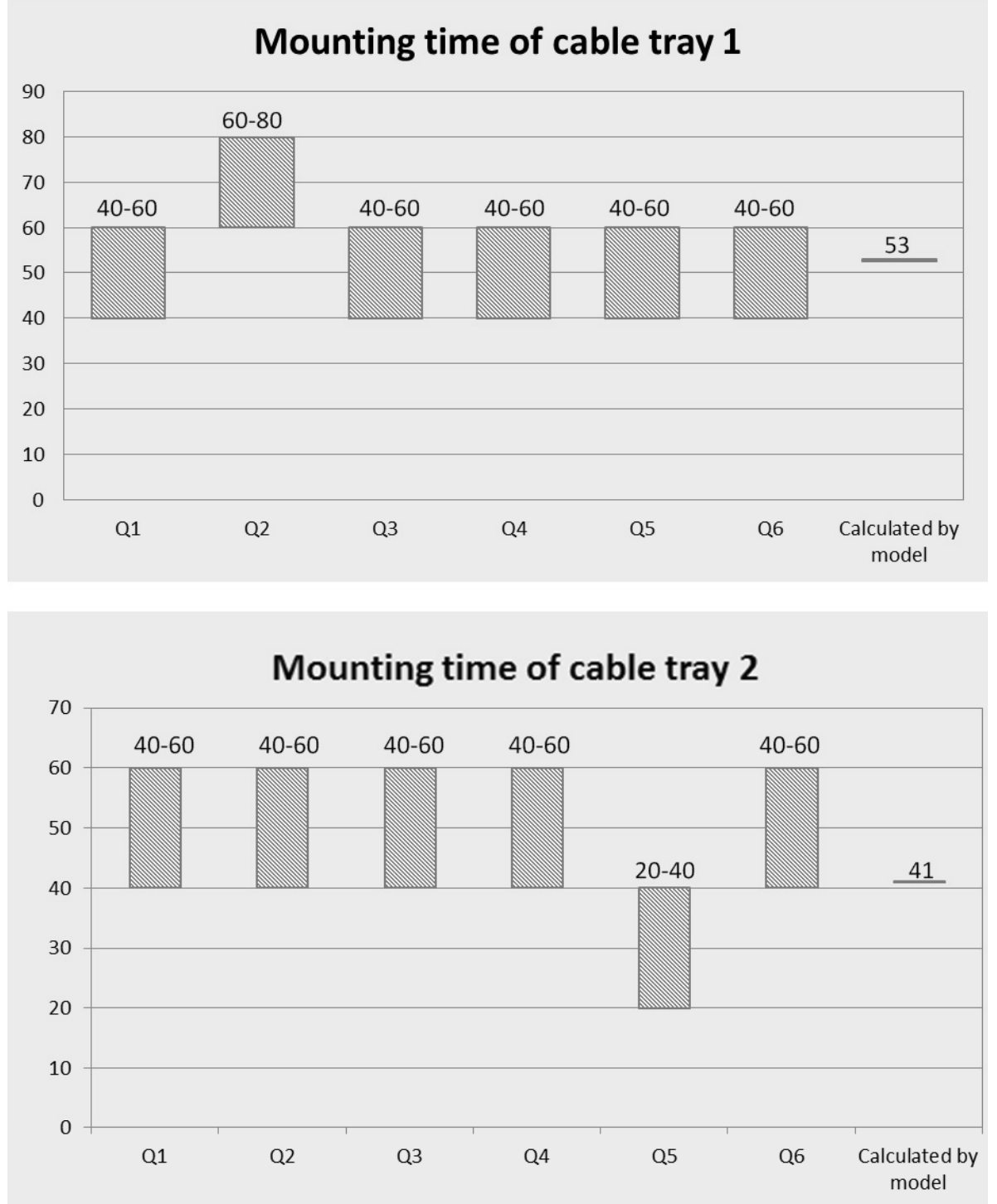

Figure 9.6: Answers from the questionnaires: estimation of mounting time of cable tray 1 and 2 


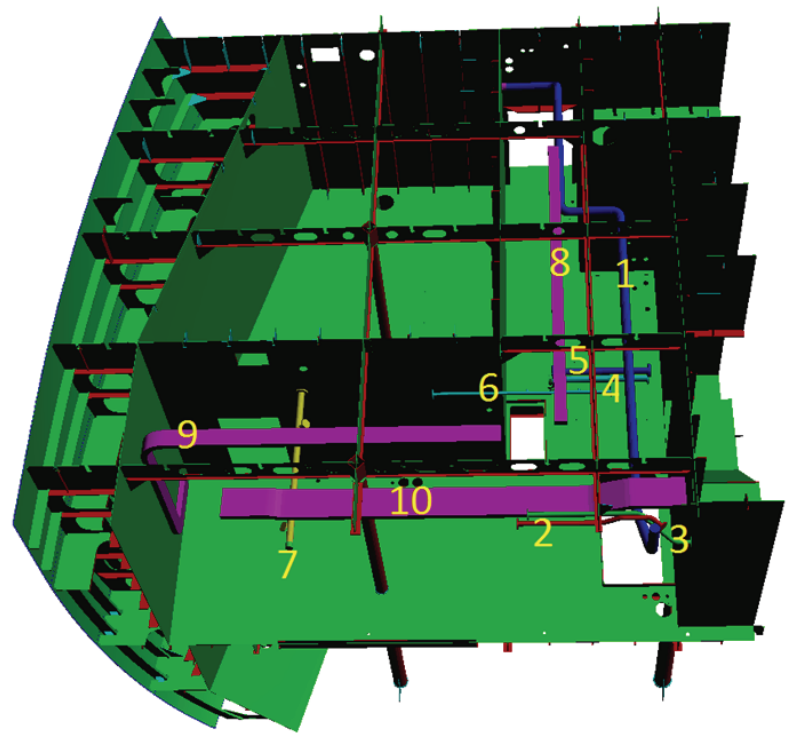

Figure 9.7: A test case with 10 components

components can be installed at the beginning or at the end. In the Downward version, component 4, 5, 6 and 7 can be disassembled at the beginning. Thus, these four components were assembled at the end. In the Upward version, component 2, 8, 9 and 10 were first removed and the first to be assembled. (In either version, components having the same color indicate that they have the same height ranking.)

As described in chapter 6.2.1, if there is no interference between one component and all other components, such a component can be removed. There are more components that can be removed at the first height iteration check compared to the number of components that can be removed in a later stage. In the 'Downward' version, more components will be installed at the end, but in the 'Upward' version more components will be installed at the beginning. This is due to the assumption that the 'Downward' disassembly order of components is the reverse order of assembling them. For the 'Upward' disassembly, there is no need of such an assumption, because the disassembly order of the components are the same as their assembly order.

When the duration is not taken into account, which version of the assembly sequence is better? Four test cases with 14, 10, 7 and 6 components were tested. When faced with the 4 assembly cases and their corresponding (upward and downward) sequences, 6 people were required to indicate which one they thought better: upward or downward. Table 9.6 shows the result of their comparison. Respondent Q5 thought both versions were feasible for the 14-component and 10-component test cases and the better one should have less duration than the other. The other 5 employees are clearly divided in their opinions on the better direction. They feel there is not too 
126 Automatic generation of assembly sequence for the planning of outfitting processes in shipbuilding

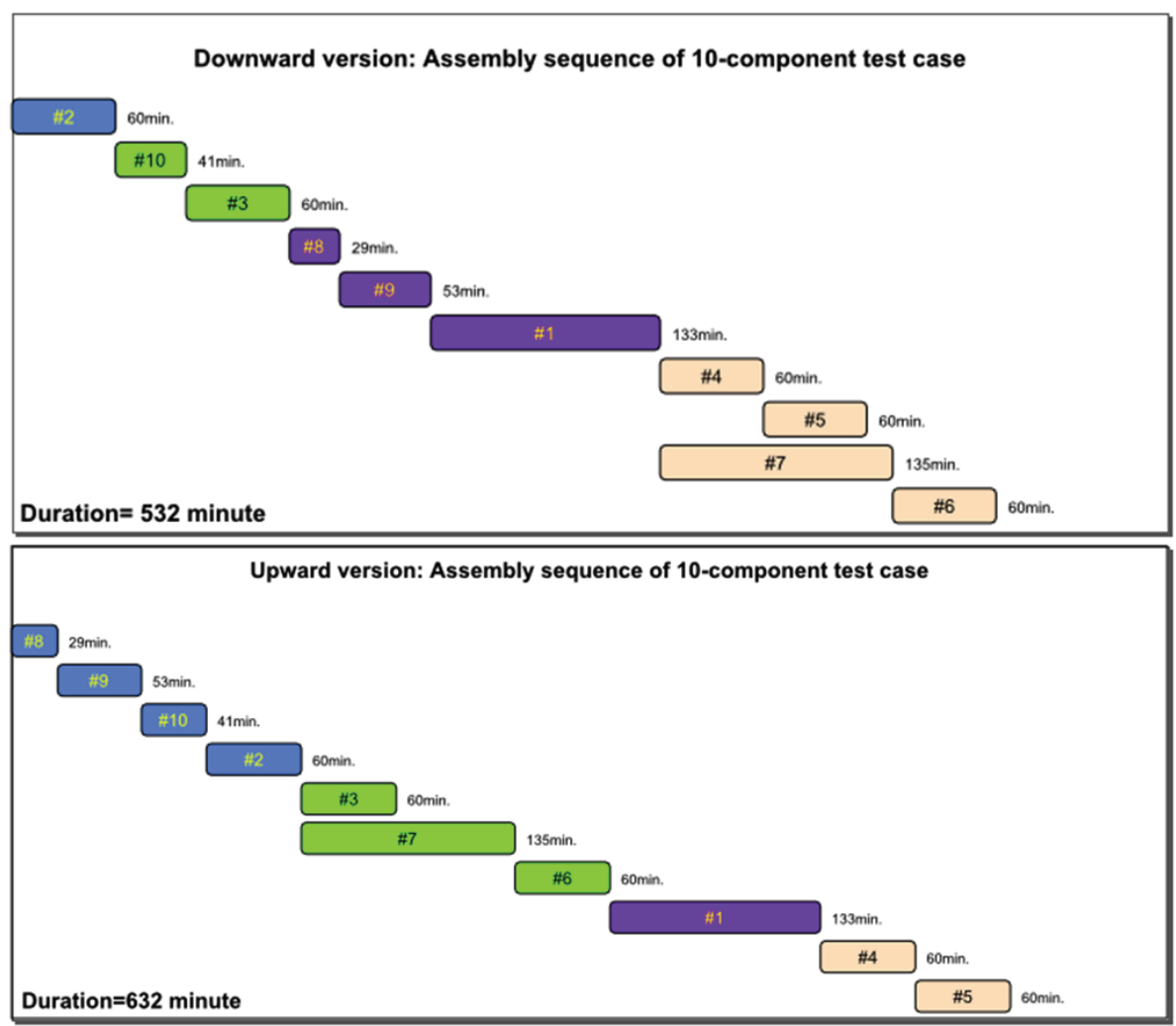

Figure 9.8: Downward and upward version: Assembly sequence of 10-component test case

much difference if either more components are assembled at the beginning or at the end of the outfitting process. Thus, the choice of the better assembly sequence hinges on the duration. The one with less duration is preferred.

\subsection{Analysis of test cases}

The previous test cases for different numbers of spools were from the same steel section of a ship- a section of an engine room work shop. Next to the analysis of the assembly sequences for that section, this chapter also includes test cases for three other sections from the same ship, two engine room sections and one wheel house section. Here the outfitting work is more complicated and can be considered representative to test the functionality of the model. Besides, the feasibility of the assembly sequence has been validated and the comparison of duration between the results from the model and the estimates from the pre-outfitting plan has also been conducted. 
Table 9.6: Choose the better assembly sequence

\begin{tabular}{ccc|cccc|cc}
\hline & \multicolumn{2}{|c|}{ 14-component } & \multicolumn{2}{|c|}{ 10-component } & \multicolumn{2}{|c|}{ 7-component } & \multicolumn{2}{|c}{ 6-component } \\
\multicolumn{2}{c|}{ Up } & Down & Up & Down & Up & Down & Up & Down \\
\hline Q1 & $\mathrm{X}$ & & $\mathrm{X}$ & & & $\mathrm{X}$ & $\mathrm{X}$ & \\
Q2 & $\mathrm{X}$ & & $\mathrm{X}$ & & & $\mathrm{X}$ & $\mathrm{X}$ & \\
Q3 & $\mathrm{X}$ & & $\mathrm{X}$ & & $\mathrm{X}$ & & & $\mathrm{X}$ \\
Q4 & & $\mathrm{X}$ & & $\mathrm{X}$ & $\mathrm{X}$ & & & $\mathrm{X}$ \\
Q5 & $\mathrm{X}$ & $\mathrm{X}$ & $\mathrm{X}$ & $\mathrm{X}$ & $\mathrm{X}$ & & & $\mathrm{X}$ \\
Q6 & & $\mathrm{X}$ & & $\mathrm{X}$ & $\mathrm{X}$ & & & $\mathrm{X}$ \\
\hline
\end{tabular}

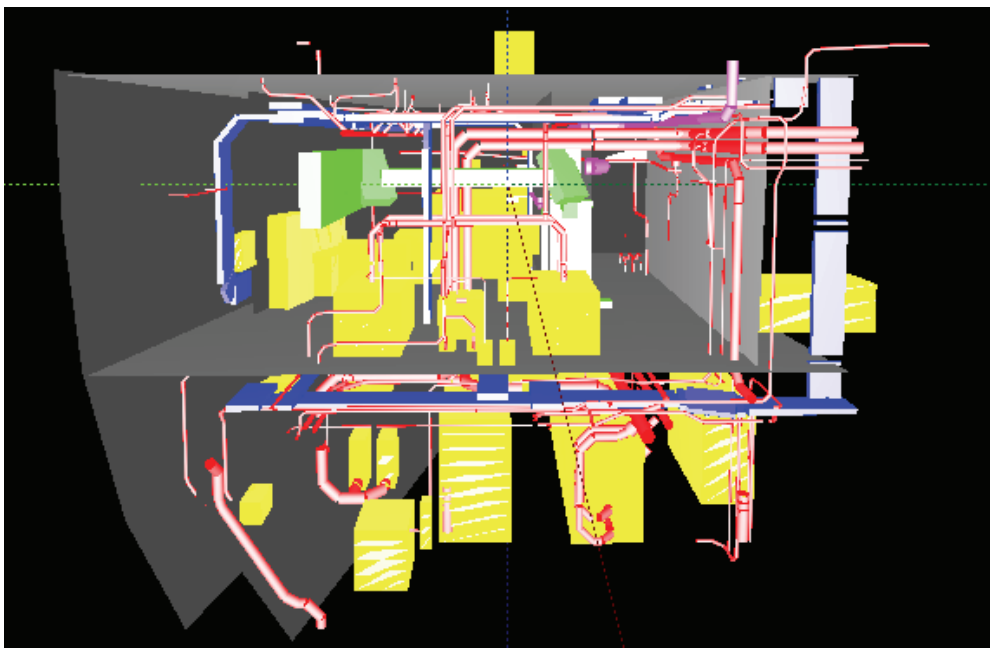

Figure 9.9: 3D visualization of the complete section $\mathrm{S} 1$

\subsubsection{Four test cases}

The section of an engine room work shop S1 for a support vessel contains 476 spools and 46 pieces of equipment to be installed during the outfitting stages. After running the model, its 3D visualization is displayed in figure $9.9^{1}$, compared with its original 3D engineering model (including the steel structure) shown in figure 9.10. The animation of its assembly sequence can be found in appendix $\mathrm{F}$.

Part of the complete assembly sequence in Gantt Chart is shown in the right hand side of figure 9.11 and the overall view of the sequence was presented in the left hand side of figure 9.11. The duration for assembling section S1 resulting from the model

\footnotetext{
${ }^{1}$ In this picture and the following ones, the steel structure in the model is only for a purpose of visualization and has not taken into account during the model development
} 
128 Automatic generation of assembly sequence for the planning of outfitting processes in shipbuilding

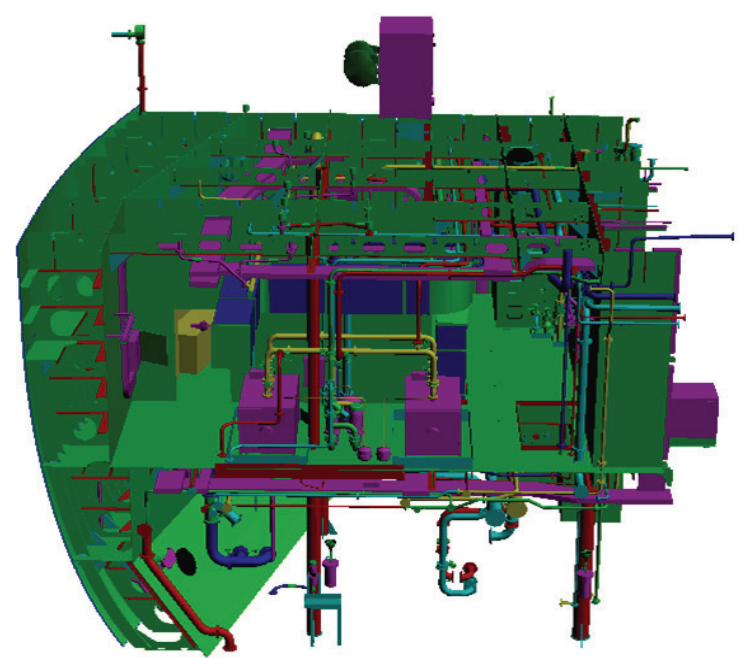

Figure 9.10: Original 3D engineering model of section S1

was compared to the value from the real pre-outfitting plan.

The same procedure has been applied to three other sections, two engine room sections (S2,S3) and one wheel house section (S4). The number of spools and pieces of equipment in these sections have been listed in table 9.7. Here the duration both of the plan as well as the model (including Downward and Upward version) was presented. It can be found that the duration of the model underestimates the time from the pre-outfitting plan by between $18 \%$ to $44 \%$.

Table 9.7: The comparison of duration

\begin{tabular}{lcccc}
\hline & S1 & S2 & S3 & S4 \\
\hline No. of spools & 476 & 427 & 410 & 219 \\
No. of piece of equipment & 46 & 35 & 26 & 3 \\
Duration-plan (hour) & 192 & 176 & 160 & 120 \\
Duration-model(D) (hour) & 120 & 98 & 106 & 98 \\
Duration-model(U) (hour) & 126 & 125 & 126 & 81 \\
\hline
\end{tabular}




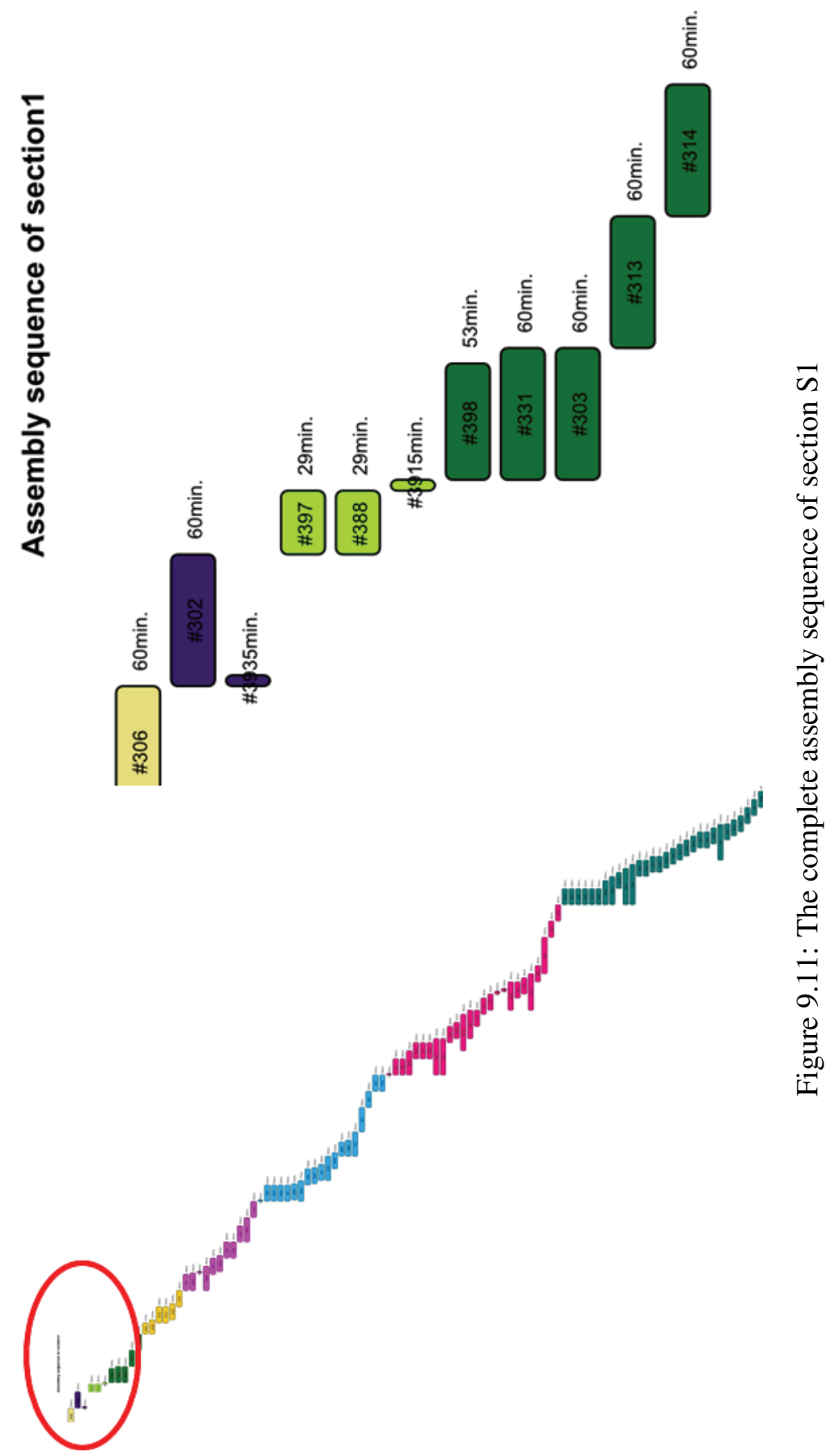


130 Automatic generation of assembly sequence for the planning of outfitting processes in shipbuilding

\subsubsection{Discussion of the assembly sequence and duration}

\section{The assembly sequence validation}

Among different validation techniques found in the literature (Sargent, 2005), face validity, i.e. asking individuals knowledgeable about the assembly work whether the model and/or its behavior are reasonable, was chosen due to the fact that neither assembly sequences on the work site nor the contextual conditions (such as organizational and procurement constraints) are recorded. To validate the feasibility of the assembly sequence, several meetings with people from different shipyards have been organized. The assembly sequence of section S1 was taken as an example to be discussed and analyzed during the meetings. The results from these meetings have been documented in the following paragraphs.

Two senior piping workers ${ }^{2}$ from a piping company were asked to participate in the first meeting. It is considered that their experience is sufficient for making judgments on the assembly sequences of test case S1. During the meeting, they put forth questions about mounting pipes, such as whether the penetrations and interferences have been considered or not. Their focus was only relevant to pipes. Concerning the assembly of other types of components, they did not possess such knowledge.

In conclusion, while being experts in their field of work, these two employees were not in a position to overview the whole outfitting work and therefore, were not able to give judgment on the assembly sequence.

The fruitful discussions were obtained from another three meetings participated by different people ${ }^{3}$. The following conclusions have been drawn from the feed-back of these experts:

Conclusion 1: In general, the sequence is physically possible;

Conclusion 2: The starting point that parts closer to the steel are preferred to be installed earlier is a good practical choice; this sequence solved the many interferences which is one of the main reasons for rework and more coordination on the work site

Conclusion 3: There are four major points in the model that are different from the real production:

1. In the model, due to the lack of steel boundary information related to the division of the ship's structure into sections, components nearby the edge of the steel structure have not been distinguished and participate in the sequence generation.

In reality, according to the convention of the Dutch shipyard where these experts work for, components located less than $50 \mathrm{~cm}$ away from the edge of the

\footnotetext{
${ }^{2}$ One has 15 years experience and the other 21 years.

${ }^{3}$ One is an outfitting coordinator, one is a piping supervisor, two are pre-outfitting coordinators, one is a project manager consulting with his planning work team
} 


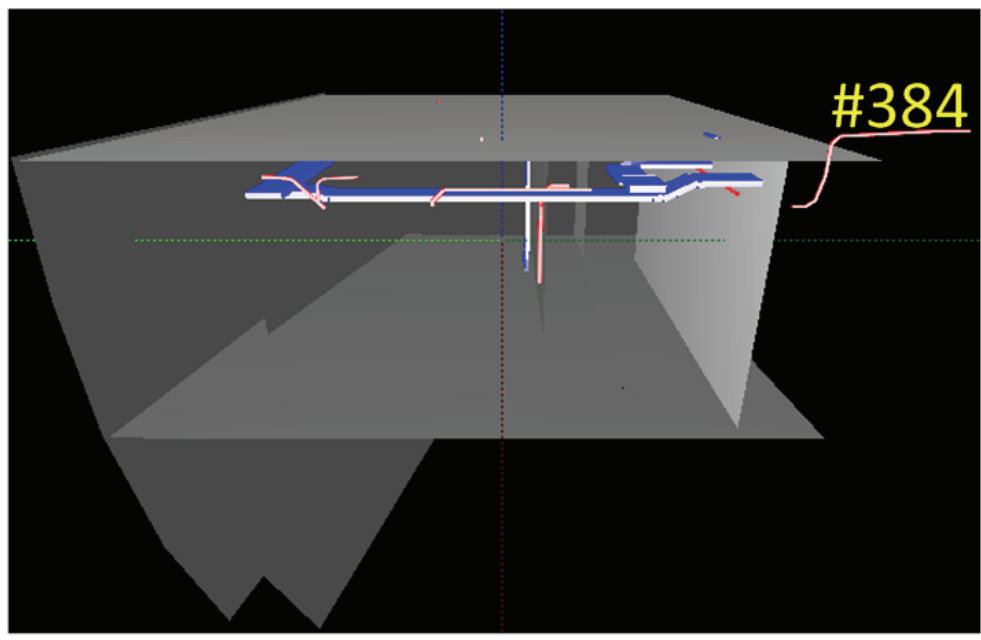

Figure 9.12: A pipe spool is near the edge of the steel section

steel section are not installed during the pre-outfitting stage. The reasons are:

- It is easy to damage them when assembling this section with its adjacent sections.

- The placement and the welding of the two sections on the slipway requires space near the edge to be empty for operational purpose.

- These components need to be connected with other components in another section. Such connections can only be completed when the other section where they are located is precisely assembled with this section.

An example in the model is shown in figure 9.12. In this test case, component $\sharp 384$ is very close to the edge of this section. In reality, it will not be installed during the pre-outfitting stage, while the model shows that it is the 18th to be assembled, far earlier than the other components, because it is closer to the upper deck. This clearance convention may not prevail in other shipyards that are able to handle section assemblies in another way. Thus, to distinguish the outfitting components near the edge of the steel structure from the rest will not be the concern of the model.

2. In reality, in most situations penetration pieces are installed earlier than the ones which are connected to them. If accessibility allows, they are preferred to be installed first during the pre-outfitting stage. It is not always done like this in the model, because the model does not distinguish the cable tray penetration pieces from other cable trays because of the limited cable tray information.

For example in figure 9.13 , in the test case, $\sharp 390$ is the cable tray penetration piece and the 349 th to be assembled based on the sequence. $\sharp 398$ is the cable 
132 Automatic generation of assembly sequence for the planning of outfitting processes in shipbuilding

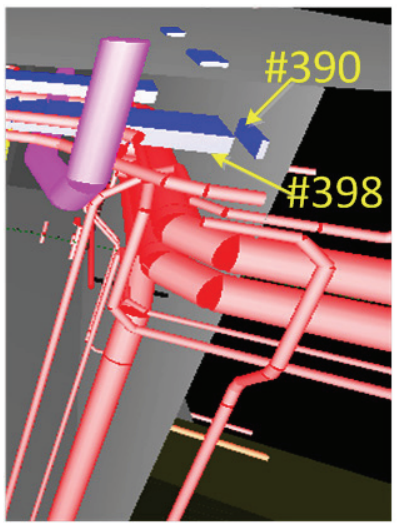

Figure 9.13: A cable tray penetration piece and its connected cable trays

tray which is connected with $\sharp 390$ and the 6th to be installed. This is different from real life. Note that in this case the small size and mass of the penetration piece cause its late assembly according to the model. Clearly, increasing the weighting of the penetration piece can reduce this problem but would violate the earlier judgments of the workers which were the basis for the weighting coefficients. This will be disccused in chapter 10.1 .

3. On the work site, components connected to equipment are assembled after the installation of that piece of equipment. However, exceptions do exist in the model.

In figure 9.14 , the sequence from the model shows that $\sharp 485$, a central heating boiler, is the 72 th to be assembled and $\sharp 7$, the pipe piece connected to $\sharp 485$, is the 35th to be assembled. This is because as described in chapter 7.1.3, the model is not able to detect which components are connected to what piece of equipment in the model and also because according to the interference detection of the model, the pipe piece is above the central heating boiler (viewed from $+Z$ direction) and its assembly should precede that of the boiler. This hardly ever happens in reality.

To trace the connections between the components and its connected pieces of equipment would require the availability of a topological model which currently is not available in shipyards. Alternatively it would require a completeness of the 3D model (no gaps) which is not practical because some gaps are deliberately used to represent e.g. spools manufactured on-site.

4. Usually, pipelines start with a penetration piece and end with either another penetration piece or equipment. Practically, the installation of pipes should follow the pipeline in order to avoid inserting one pipe spool in-between two 


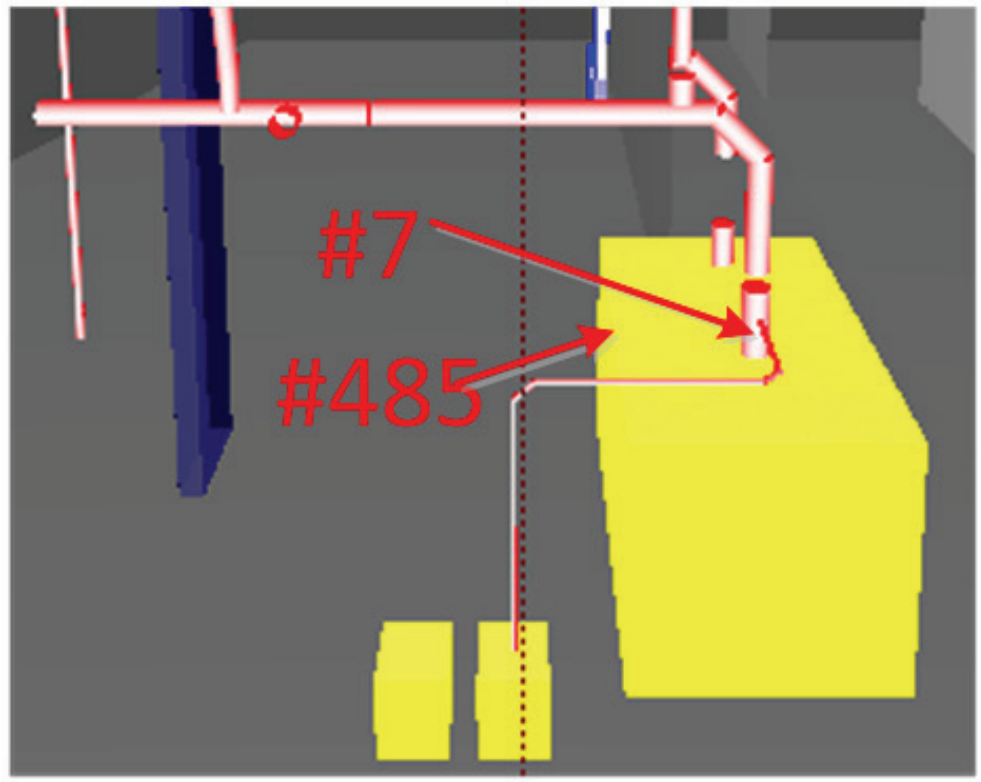

Figure 9.14: A central heating boiler and its connected spool

other pipe spools. However, according to the assembly sequence in the model, insertion operations do occur.

For example, in figure 9.15, there are two pipelines in the model. According to the sequence from the model, the assembly of components in the two lines does not take place by following the pipeline. Figure 9.16 shows that pipe spools in the middle of the two pipelines are inserted between other spools. This phenomenon is caused by the fact that at present, the model cannot detect which pipe spool is connected to which in a single pipeline due to the incompleteness of xml input data, although many efforts have been put into such detection. Again, a topological model would be the preferred solution.

\section{Duration discussion}

Apparently, table 9.7 shows that important deviations of the duration between the plan and the model do exist. In order to find the possible reasons that caused the deviations and provide a detailed look at the assembly sequences, two employees from the production department of the yard ${ }^{4}$ were asked to join the discussions. One was the supervisor of piping work and the other the outfitting coordinator. They not only gave the feedback of the assembly sequences of test case S1 but also explained

\footnotetext{
${ }^{4}$ It is the same shipyard that provided the test case data for the research and also accomplished the production work of these test cases.
} 
134 Automatic generation of assembly sequence for the planning of outfitting processes in shipbuilding

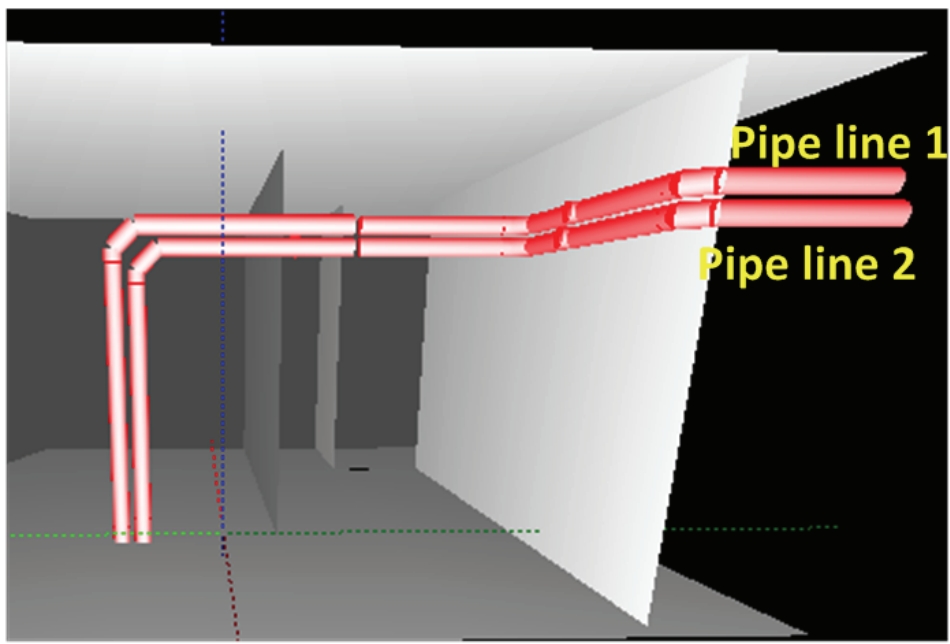

Figure 9.15: Two pipelines in the model
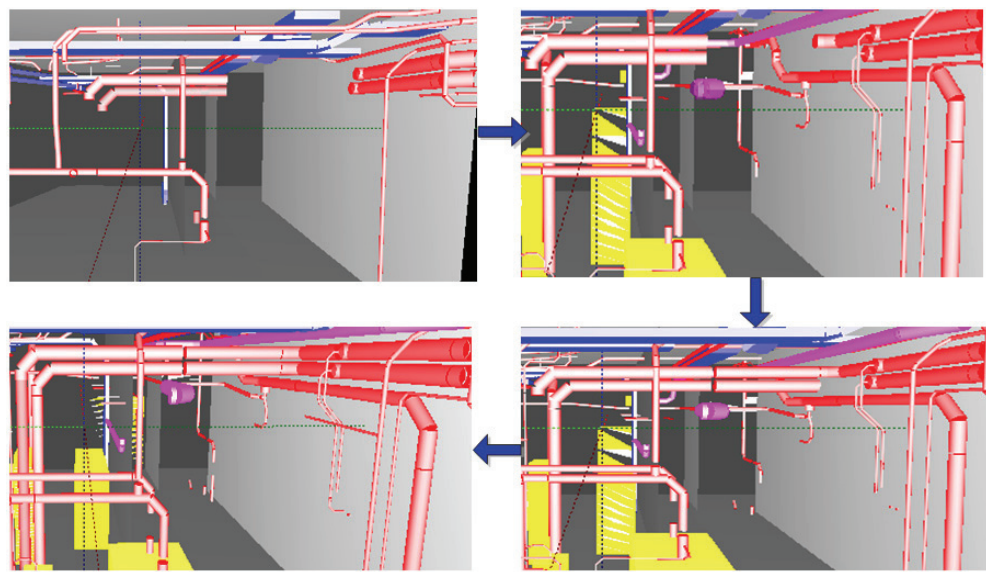

Figure 9.16: The assembly order of pipe spools on the two pipelines 
the duration generated by the pre-outfitting plan. The conclusions from this meeting have been listed as follows.

The reasons to cause the deviations between the duration of the plan and that of the model could be:

- Time spent on doing small iron work has been considered in the section preoutfitting plan.

Small iron work like stairs, handrails, foundations of heavy equipment shown in figure 9.17 is often regarded as part of steel construction as we have pointed out in chapter 2.4. The work to mount them could be proceeding at the same time with the pre-outfitting work. It influences the pre-outfitting work efficiency. For example, the welding work of mounting a foundation may prevent the assembly of a pipe spool nearby. This factor has been taken into account when planners produce the pre-outfitting plan based on their experience. It clearly leads to a longer duration for the real life plan.

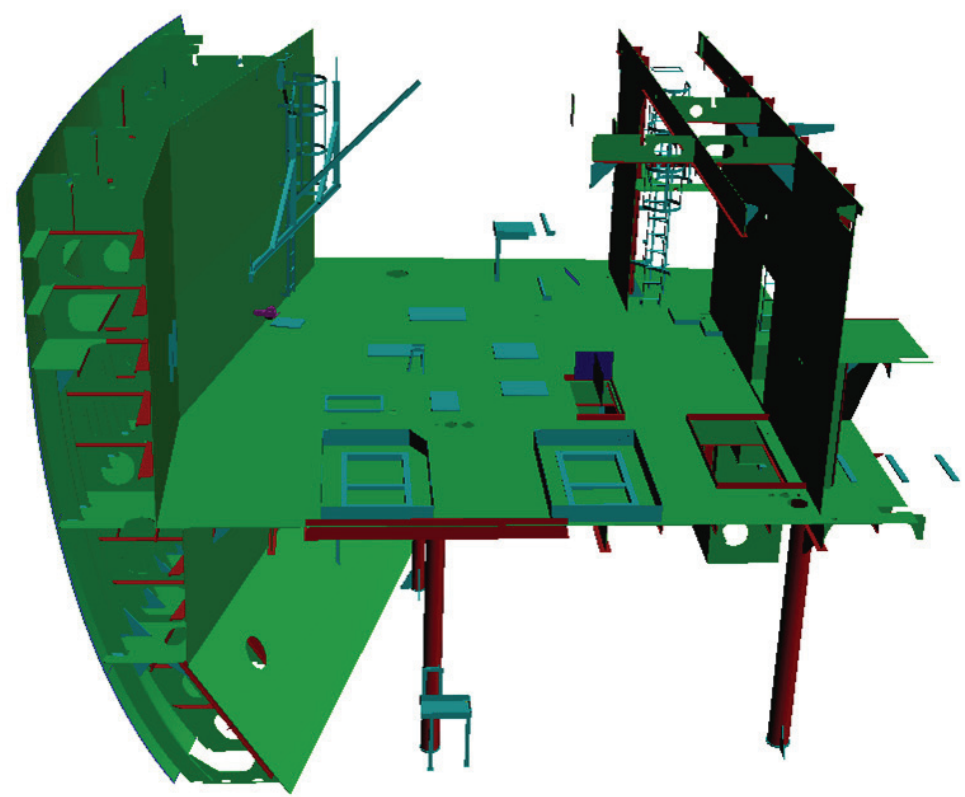

Figure 9.17: Small iron work of the test case S1 (not included in the assembly sequence generation model)

- Another reason to cause the deviations is the time spent on shooting pins (or studs) on the steel parts for the purpose of insulation. Figure 9.18 displays the area that should be insulated in the test case S1. The insulation pins were shot in the steel plates of this area during the pre-outfitting stage. 


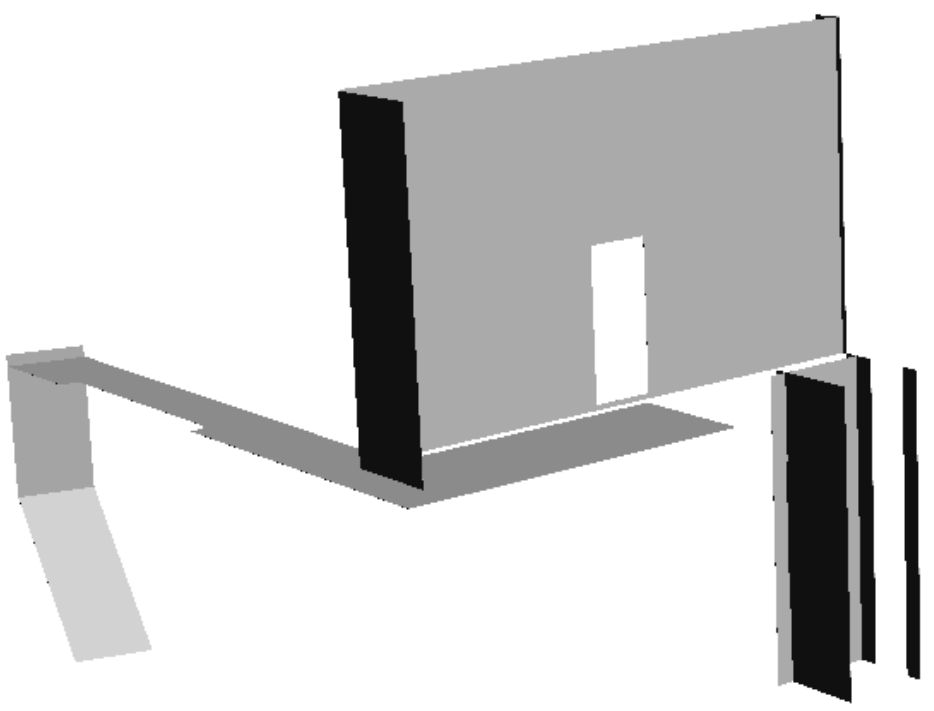

Figure 9.18: Insulation area of the test case S1

- The time necessary for turning sections from the upside down position to the upright position is also included in the pre-outfitting plan.

Apart from double bottom sections which are built directly on the slipway on the upright position, most steel sections are built in the upside down position in specific section building halls. When a certain percentage of the steel construction work is finished, the assembly of outfitting components which are closer to the ceilings will start. At a certain stage when such kind of assembly work has been finished, the section needs to be turned to the upright position so that the outfitting components closer to the floors can be added in. Changing the position of a section normally requires 1-2 days, because it requires cleaning work, dismantling and (re)building of scaffoldings, and also waiting time for the availability of the cranes.

In reality, there is no record of the order in which the outfitting components were assembled. It is also not possible to get the real duration of assembling pipes, cable trays, HVAC and equipment of a certain section, which has been at best roughly recorded by different subcontracted companies and is considered as confidential. Here, we take an indirect way to deduce the duration of assembling pipes, cable trays, HVAC and equipment from the pre-outfitting plan.

The planner in the yard was asked to estimate the duration spent on small iron work, shooting insulation pins and turning sections. In theory, the duration of assembling pipes, cable trays, HVAC and equipment from the pre-outfitting 
plan is the result of extracting this estimated duration from the pre-outfitting plan. However, in real life, as emphasized by the planner, the small iron work is carried out at the same time with the pre-outfitting work and it is incorrect to simply extract its duration the planning time. Shooting insulation pins and turning sections hardly involve (and are separated from) any pre-outfitting work. In this case it is rather safe to extract their duration from the planning time. Thus, the durations of assembling pipes, cable trays, HVAC and equipment for four test cases are obtained by deducting the duration of shooting insulation pins and turning sections from the planning time. They are listed in in row 1.b in table 9.8.

- The pre-outfitting plan hardly takes the installation of equipment into consideration. Without equipment installation, the duration of the model is listed in row 2.b in table 9.8 and is in general less than the duration including the equipment installation.

Table 9.8: The duration of four test cases under different scenarios

\begin{tabular}{lcccc}
\hline & S1 & S2 & S3 & S4 \\
\hline 1.a:Duration-plan (hour) & 192 & 176 & 160 & 120 \\
1.b:Duration-plan after deduction (hour) & 172 & 159 & 144 & 108 \\
& & & & \\
& 120 & 98 & 106 & 98 \\
2.a:Duration-model (hour) & 119 & 95 & 97 & 96 \\
2.b:Duration-model without equipment (hour) & 186 & 160 & 156 & 112 \\
2.c:Duration- model after resource leveling (hour) & 186 \\
\hline
\end{tabular}

- According to the model, too many components can be assembled simultaneously. The more components are assembled at the same time, the less duration is required. This could be the reason that the duration from the model is far less than given by the plan.

In the real world, normally at the beginning of pre-outfitting work, they need 2 teams in a section; later on, at the maximum, they may need 3-4 teams. The high peak of requirement for work teams from the model reaches to 25 teams. This is not practical. It implies the necessity of the resource leveling, which has already been discussed in chapter 8.2.

To have a quick look at how the limitation of the number of teams influence the duration, we simplify the problem by:

- supposing that two teams work for the first half of the duration and four teams work for the second half of the duration;

- allowing that man hours obtained from the model still remain the same. 
138 Automatic generation of assembly sequence for the planning of outfitting processes in shipbuilding

Thus new durations for four test cases after resource leveling can be calculated and these are listed in row 2.c in table 9.8. Compared to the durations of the model, they are much closer to the planning time. This simplification by no means precisely reflects how the labor resource has been distributed in real assembly processes, however it indeed sticks to the fact that normally, 2 teams and maximum 4 teams work on the site at the same time and also follows the realistic assembly sequence of the model. The new duration in row 2.c is considered as reasonable.

- It is not certain that the planned duration equals the realized duration since no detailed records are kept.

In table 9.8, the durations after deduction from the pre-outfitting plan are the closest values to the reality and marked in red in row 1.b. These values are compared to the durations without equipment installation from the model which are also marked in red. It can be found that the average percentage of duration reduction by the model is $28.5 \%$. In conclusion, the assembly sequence generated by the model cannot be firmly validated through the comparison with the real data. However, the feedback from the meetings suggest that the collision-free assembly sequence and the insight that the model offers to all co-makers in this joint outfitting operations may lead to the reduction of the total outfitting duration.

\subsection{Limitation of the model and possible improvements}

The introduction of the assembly sequence generation model for outfitting processes was aimed at the integration of assembly planning across the various disciplines and the reduction of coordination efforts on the work site. To an outsider the outfitting processes are complicated and seem chaotic. Little of the ongoing processes is recorded. Data is scarce. Modeling of such a process is extensive and complicated. The main obstacle has been to create a model that provides a sufficiently detailed insight into the assembly process. The use of geometrical attributes available in the CAD model to capture the assembly knowledge has led to conflict-free and acceptable sequences. To obtain the complete representation of outfitting elements and the clear configuration of their intertwined relationships, more investigations on the work site and into adequate engineering data are still required. Starting from the aspects of functional performance, this section dwells on the identification of the limitations of the model and its further improvements.

\subsubsection{Obtain the steel structure information}

As mentioned in section 8.4, the current exclusion of steel structure information hinders the model to be developed in a more realistic way. In a section, outfitting components closest to the steel structure are normally assembled first in the pre-outfitting 
stage when the section is either in the upright position or the upside down position. The crucial factor to decide the mounting order is the relative distance, in $\mathrm{X}, \mathrm{Y}$ or $\mathrm{Z}$ direction, between the outfitting components and their nearest steel structure.

So far, when detecting if there is interference between two outfitting components, the model only compares their global $\mathrm{Z}$ coordinates instead of their relative distances between the steel structure and themselves. This leads to the possibility that components closest to the bulkhead or steel walls may not be the first ones to be installed just because their global $\mathrm{Z}$ coordinates dominate the model's decision making.

Thus, possibilities for further optimization are:

- Obtain the steel structure information from the 3D model and integrate the relevant data into the model. It is believed that the data preparation model introduced in section 7.1 is able to reorganize the steel structure data in such a way that it can be accessed by the model.

- Categorize outfitting components based on their proximity to steel structural elements. The normal to such a piece of steel structure decides in what direction to detect interferences among its proximate outfitting components. For this, it is necessary to know which outfitting component is in the proximity of a certain piece of steel structure. If a component has the same proximity to more than one piece of steel structure, its proximate steel structure should be decided by where the major part of this component locates.

- For the group of components belonging to this particular steel element, build up the interference matrices along the normal to the steel structural element.

- Test the possibility to assemble all the components nearest to the steel structure in parallel, i.e., the installation starts from the periphery of the steel structure until it reaches the spatial center of that section. It implies that 6 components can be mounted at the same time because a closed compartment possesses 6 sides, ceiling, floor and four walls. However, this may not be acceptable from the point of view of safety because the assembly work on the ceiling may cause greater danger to people who work on the floor area. Thus, the possibility to apply this idea requires further consideration. Limiting the number of teams to a practical value of 2 to 4 would eliminate this problem.

\subsubsection{Installation of equipment}

The way to generate the mounting sequence for equipment needs more investigation. In the model, equipment is treated the same as other outfitting components, i.e. its position, weight, material and size decide the mounting order. In reality, this assumption is oversimplified. In table 9.9, the difference between the installation of equipment and other outfitting components, like pipes, HVAC ducts, is described 
140 Automatic generation of assembly sequence for the planning of outfitting processes in shipbuilding

from the aspects of engineering, the plan of installation and production. It shows that the installation of equipment depends on more factors than only the other outfitting components. For example, to make sure that a bigger piece of equipment can be placed into the compartment, the section division plan and hull erection plan provides the specific period during which the section is still open and allows to be accessed by the equipment.

Table 9.9: The difference in input sources for determining the mounting order and mounting time of equipment and other outfitting components

\begin{tabular}{|c|c|c|}
\hline & Equipment & Other outfitting components \\
\hline \multicolumn{3}{|l|}{ Engineering } \\
\hline 3D model & Rough boundary of box & Detailed geometrical attributes \\
\hline \multicolumn{3}{|l|}{ Plan of installation } \\
\hline \multirow[t]{7}{*}{ Input } & General arrangement; & Combination drawings; \\
\hline & Section division plan; & Specifications \\
\hline & Pre-outfitting plan; & \\
\hline & Outfitting plan; & \\
\hline & Hull erection plan; & \\
\hline & A list of components; & \\
\hline & Compartment numbers, doors and Hatches & \\
\hline \multicolumn{3}{|l|}{ Production } \\
\hline Installation procedure & Place inside of the compartment+ install & Directly install \\
\hline Relationship with steel structure & Compartment+ deck & Relative distance \\
\hline
\end{tabular}

The obstacles for the model to generate a more realistic assembly sequence for equipment are as follows:

- Lack of integration of the information about the equipment's connection points with other components and their locations, for example in which compartment and on which deck. Although this information is presented in the form of the DXF file in the database, integrating them in the way that they can be accessed by the sequence generation model needs further attention. For this more associative models, logically connecting geometrical, geographical and topological information are useful.

- Lack of knowledge of how the equipment's location in the steel structure and its geometrical attributes influence its mounting order. For example, some equipment could be installed in the pre-outfitting stage, but if the capacity of the crane used to transport the section to the painting hall or slipway, is taxed beyond its limit, it is not allowed to be put into the section. So it must be installed on-board. If its size is not too big and can be put into the compartment through the doors, then it does not matter (apart from possible impacts on production efficiency) whether this equipment is installed in the earlier stage when the compartment is still open or in the later stage on the slipway.

- Lack of insight in how the installation of other outfitting components, like pipes 
and HVAC, influences the installation of equipment. The placement of equipment requires the floor print nearby the equipment's location to be sufficiently clean to allow the maneuvering of equipment and efficient access for workers.

Considering the above barriers, which seem to hinder unfolding the complicated relationships involved in the installation of equipment, we have tried to picture the roadmap for subsequent investigations relative to equipment mounting. As shown in figure 9.19, it suggests:

- Investigate which factors influence the equipment mounting order.

- Reorganize the input information in a way that can be processed by the model. There are five main tasks in the equipment data preparation model:

First, assign the start and finish date of pre-outfitting and hull erection for each section. This can easily be derived from planning documents.

Second, couple each compartment with its sections and specify what equipment is in which sections. For example, in figure 9.20, Equipment 1 is located in section 101 and the neighboring sections of 101 will be identified. This leads to the composition of the compartment where equipment 1 is.

Third, detect the open-sides of a section which provides access for the equipment. The sizes of openings, whether they are a door or a bulk head in another section, indicate the possibility to install the equipment during pre-outfitting or on-board outfitting. If the size of equipment is smaller than the smallest opening, it does not strictly matter during which stage it should be installed.

Fourth, filter the equipment list with respect to the procurement planning to ensure that a component is delivered in time for the intended mounting date.

Fifth, collect the geometrical data of equipment from 3D model, including its size, weight, connection points and exact position.

- Establish a more detailed model for how both steel structure and other outfitting components influence the installation of equipment.

- Obtain a more realistic equipment installation sequence and validate it.

\subsection{Conclusion}

The application cases and validation analyses in this chapter show the potential and possibilities of an automatic assembly sequence generation approach. It shows that with the currently available engineering data a feasible sequence can be automatically generated. As described in chapter 4.1.2, the feasible assembly sequence is the one which satisfies all technical constraints, but it is by no means a proper sequence since 
142 Automatic generation of assembly sequence for the planning of outfitting processes in shipbuilding

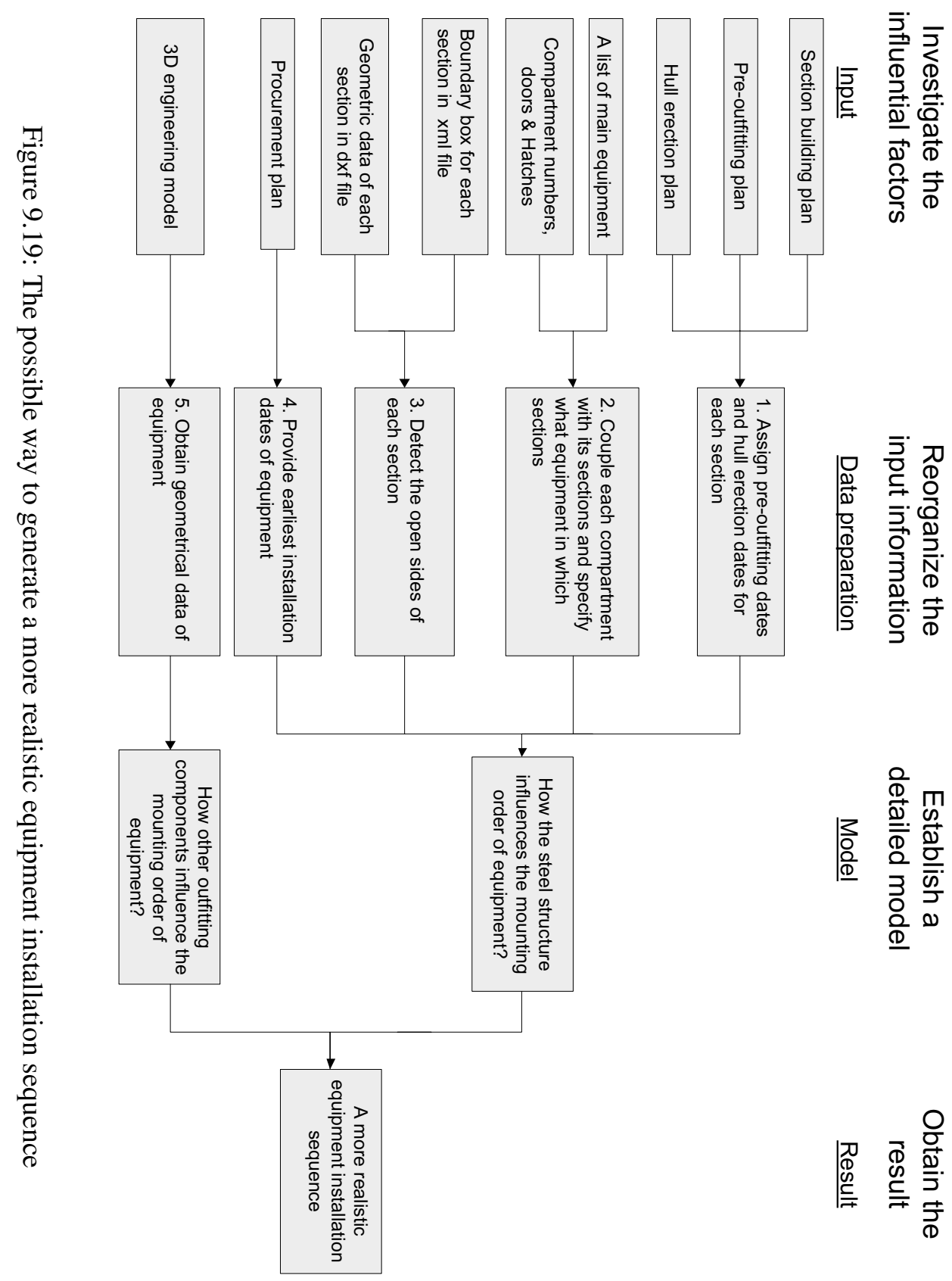




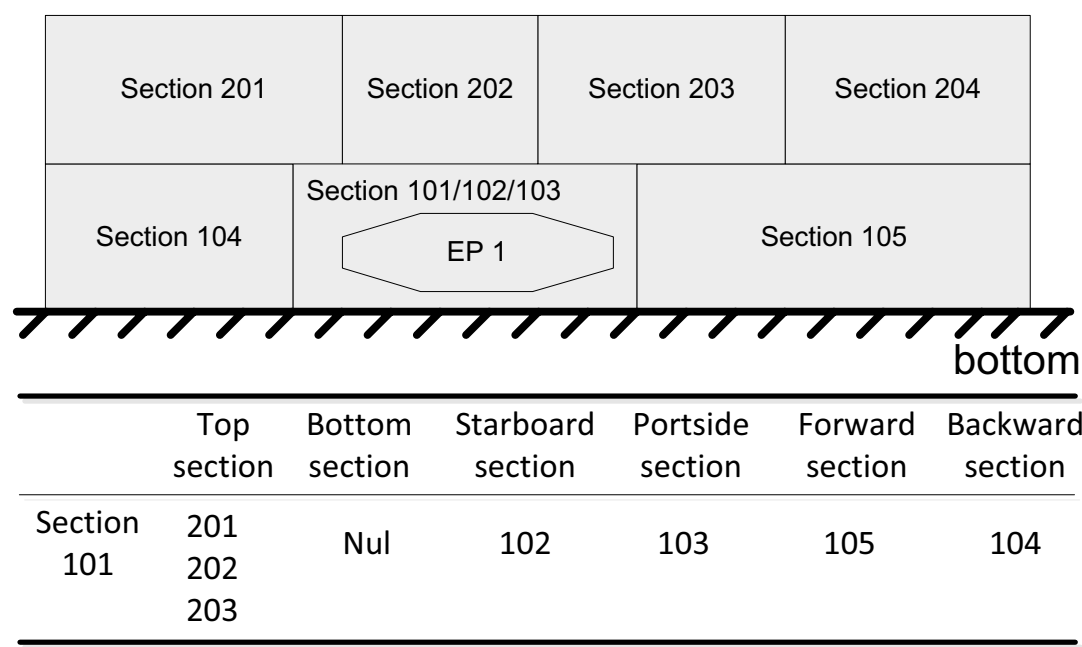

Figure 9.20: Couple compartments with sections

it is generated under the assumption that the various resources, such as drawings, material, equipment and workers with certain skills, etc. required to perform the activities are always available. The proper sequence should not only be interferencefree but have shorter duration with superior resource utilization. The validation of the mounting time indicates, but does not prove, that the estimates for the mounting time of assembling different type of components are rather realistic.

Discussion of the model's result shows that it is very difficult to consider all technical and organizational aspects of outfitting processes at the same time. In fact, given all the complexities, it is gratifying that the method yields already useful assembly sequences that provide a basis for a better planning method. Nevertheless, before the developed model can be implemented on the worksite, additional effort will be necessary both in gathering validation data and completing the model (including the integration of steel structure). Notwithstanding the evident shortcomings of the presented modeling method and tool, it already provides planners and process controllers with a powerful aid for guiding outfitting process analysis, planning and improvement. It already allows for generation of interference-free and integral assembly sequences including their durations. The behavior of the model will further improve and become more realistic by implementation of all three dimensions of interference-detection, an improved equipment-mounting algorithm and the integration of steel structure information. 



\section{Chapter 10}

\section{Sensitivity Analysis}

In many engineering and science disciplines, computer models or codes are used to simulate complex physical or organizational processes. Ideally, such a computer model is a perfect mathematical representation of the real system. If so, when setting the input variables used in the field, the computer output and the field measurement match exactly. In addition, one or more of the input variables of the computer model maybe unknown or immeasurable in the physical system. Thus, the output from a limited number of computer runs are compared to the field observation or measurement to adjust these input variables. Sensitivity analysis (SA) is the study of how the variation (uncertainty) in the output of a statistical model can be attributed to different variations in the inputs of the model (Saltelli and Ratto, 2008). Put into another way, it is a technique for systematically changing variables in a model to determine the effects of such changes. and evaluate them

When the model is applied to capture the assembly knowledge and generate the assembly sequences in outfitting processes, some inputs are known and specified, e.g. the geometrical information (position, weights and material) of components. Others are unknown but represent knowledge that can be easily made explicit, e.g. the mounting time of components and the minimum work distance. And some are largely unknown and represent tacit knowledge, such as weighting factors (for position, material, weight, size and penetration). In this case, physical observations of the system are used to learn about the unknown parameters. The sensitivity analysis helps to increase the understanding of relationships between input and output variables and thus, supports the decision making by testing the robustness of the assembly sequences.

The purpose of this chapter is to outline the procedures of carrying out the sensitivity analysis for the following input variables used in the model:

- Weighting coefficients, i.e. the relative importance of components' physical attributes such that a 'goodness' of fit is obtained. We try to detect the sensitivity of the results to each of the physical attributes. 
146 Automatic generation of assembly sequence for the planning of outfitting processes in shipbuilding

Position

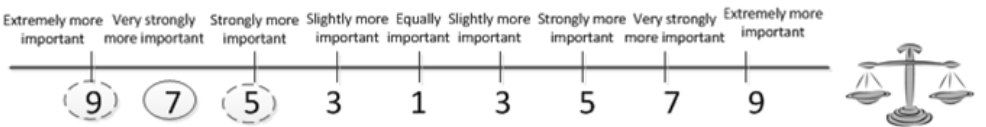

Figure 10.1: The comparison of importance levels

- Minimum work distance estimation and how it influences the total duration time and the maximum number of components that could be installed in parallel.

- Two versions, upward and downward, of assembly sequences.

\subsection{The sensitivity of the weighting coefficients}

As mentioned in chapter 6.3, weighting coefficients are used to measure the relative importance of each physical attribute. The input of the AHP method was the judgment of the relative importance between any two physical attributes, evaluated by foremen and workers based on their experience. As long as the CI is less than $10 \%$, the subjective judgments are regarded as acceptable. How their changes influence the final assembly sequence will be discussed in this section.

\subsubsection{Explore the acceptable weighting coefficients}

To investigate how the change of weighting coefficients affects the result, we first need to explore their acceptable values. In the research, this links to how they were derived.

The importance level of a specific physical attribute, compared to any other attribute, is varied between -4 levels and +4 levels. The minus-levels are 1/9, 1/7, 1/5, and 1/3 the importance; the plus-levels are 3,5, 7 and 9 times the importance. Our initial evaluation led to believe that a spool's 'position' in the steel structure was approximately 7 times more important than its 'weight' attribute when deciding its assembly order. The example in figure 10.1 is used to illustrate how to change the importance level of 'position' attribute, compared to 'weight' attribute. It is either increased from 7 to 9 or decreased from 7 to 5 .

Now, this importance has become one level higher or one level lower, by setting the value as 9 or 5. The Pairwise Comparison Matrix, used to express priorities 
among the physical attributes, originally was as follows:

$\left[\begin{array}{cccccc}\text { Attribute } & \text { Position } & \text { Material } & \text { Weight } & \text { Size } & \text { Penetration } \\ \text { Position } & 1 & 9 & 7 & 5 & 1 \\ \text { Material } & 1 / 9 & 1 & 1 / 5 & 1 / 3 & 1 / 7 \\ \text { Weight } & 1 / 7 & 5 & 1 & 1 & 1 / 3 \\ \text { Size } & 1 / 5 & 3 & 1 & 1 & 1 / 5 \\ \text { Penetration } & 1 & 7 & 3 & 5 & 1\end{array}\right]$

Increasing the importance level of the other parameter relative to 'position', it becomes:

$\left[\begin{array}{cccccc}\text { Attribute } & \text { Position } & \text { Material } & \text { Weight } & \text { Size } & \text { Penetration } \\ \text { Position } & 1 & 9 & 9 & 7 & 3 \\ \text { Material } & 1 / 9 & 1 & 1 / 5 & 1 / 3 & 1 / 7 \\ \text { Weight } & 1 / 9 & 5 & 1 & 1 & 1 / 3 \\ \text { Size } & 1 / 7 & 3 & 1 & 1 & 1 / 5 \\ \text { Penetration } & 1 / 3 & 7 & 3 & 5 & 1\end{array}\right]$

Previously, the position was 7 times more important than weight, 5 times more than the size and as important as the penetration. After increasing its importance by one level, it becomes 9, 7 and 3 times as important, as indicated by the red color in the matrix. For the material attribute, since the position is already 9 times more important, there is no space to further decrease its importance level. Thus, the number 9 remains the same after the increase. The same principle can also be applied to the number 1/9, which signifies the minimum measurement.

Decreasing the importance level, it becomes:

$\left[\begin{array}{cccccc}\text { Attribute } & \text { Position } & \text { Material } & \text { Weight } & \text { Size } & \text { Penetration } \\ \text { Position } & 1 & 7 & 5 & 3 & 1 / 3 \\ \text { Material } & 1 / 7 & 1 & 1 / 5 & 1 / 3 & 1 / 7 \\ \text { Weight } & 1 / 5 & 5 & 1 & 1 & 1 / 3 \\ \text { Size } & 1 / 3 & 3 & 1 & 1 & 1 / 5 \\ \text { Penetration } & 3 & 7 & 3 & 5 & 1\end{array}\right]$

The different weighting coefficients were only regarded as being acceptable when their corresponding CI value was less than $10 \%$. For example in table 10.1, CI coefficients derived for +3 and +4 importance levels of the 'position' are discarded because in both cases the CI, colored red in the table, exceeds the limit of $10 \%$. The same principle is applied to other physical attributes.

\subsubsection{Measure the sensitivity}

Once the acceptable weighting coefficients have been derived, the next step is to measure their sensitivities. Here, the assembly sequence generated under the originally 
148 Automatic generation of assembly sequence for the planning of outfitting processes in shipbuilding

Table 10.1: Position attribute: Weighting Coefficients under different importance level

\begin{tabular}{cccccccccc}
\hline & Position-4 & Position-3 & Position-2 & Position-1 & Original & Position+1 & Position+2 & Position+3 & Position+4 \\
\hline Position & 0,0505 & 0,099 & 0,1888 & 0,2952 & 0,4339 & 0,5553 & 0,6107 & 0,6403 & 0,6636 \\
Material & 0,0535 & 0,0456 & 0,0411 & 0,0375 & 0,0345 & 0,0328 & 0,0312 & 0,0301 & 0,0292 \\
Weight & 0,1856 & 0,1562 & 0,1297 & 0,1145 & 0,1043 & 0,0898 & 0,0825 & 0,0778 & 0,0738 \\
Size & 0,1705 & 0,1629 & 0,125 & 0,0964 & 0,0868 & 0,0744 & 0,0656 & 0,0621 & 0,0592 \\
Penetration & 0,5399 & 0,5362 & 0,5154 & 0,4563 & 0,34 & 0,2477 & 0,21 & 0,1896 & 0,1743 \\
CI & 0,0363 & 0,0701 & 0,0893 & 0,0812 & 0,0479 & 0,0657 & 0,09 & 0,1158 & 0,1407 \\
\hline
\end{tabular}

obtained weighting coefficients was chosen as a reference. The sensitivity of the assembly sequence to a certain physical attribute is measured by how many components change their position in the reference assembly order.

The position attribute is chosen as an example to describe how its sensitivity is measured. The assembly sequence of the 14 components test case, described in chapter 8.1, was set as the reference to measure how new sequences deviate from the original one. The test case is shown in figure 10.2.

In table 10.2, assembly sequences of these 14 components are generated for the different importance levels. The row of 'No. of moved components' indicates the number of components, whose assembly orders compared to the standard one have been changed in each new sequence. In 'moved steps', one step means that the assembly of a certain spool takes place either one step earlier or one step later compared to the original sequence. For example, in sequence 'Position-1', the assembly order of component 10, colored red in table 10.2, changes from the original eighth to the sixth. There are two steps in difference. The assembly order of component 7 changes from the sixth to the ninth with three steps in difference. The change of the assembly order also happens to components 6 and 4. Thus, for this case, 'No. of moved components' is 4 and 'Moved steps' is 8 .

\subsubsection{Analyze the sensitivity}

By the comparison of the No. of moved components and moved steps, the analysis of the sensitivity of the assembly sequence to each physical attribute is provided. We take the same example of the 14-component case to illustrate it.

\section{Position attribute}

Position plays a significant role in determining the assembly sequence. As its importance level decreases, the assembly order deviation becomes larger, reflected by the increase of moved steps. However, the resulting sequence is not reasonable anymore because of bad accessibility. For example, in the sequence generated with the 'position-4' level, component 4 is installed after component 1 and 2. In real life, this takes place the other way around because component 4 is closer to the ceiling. 


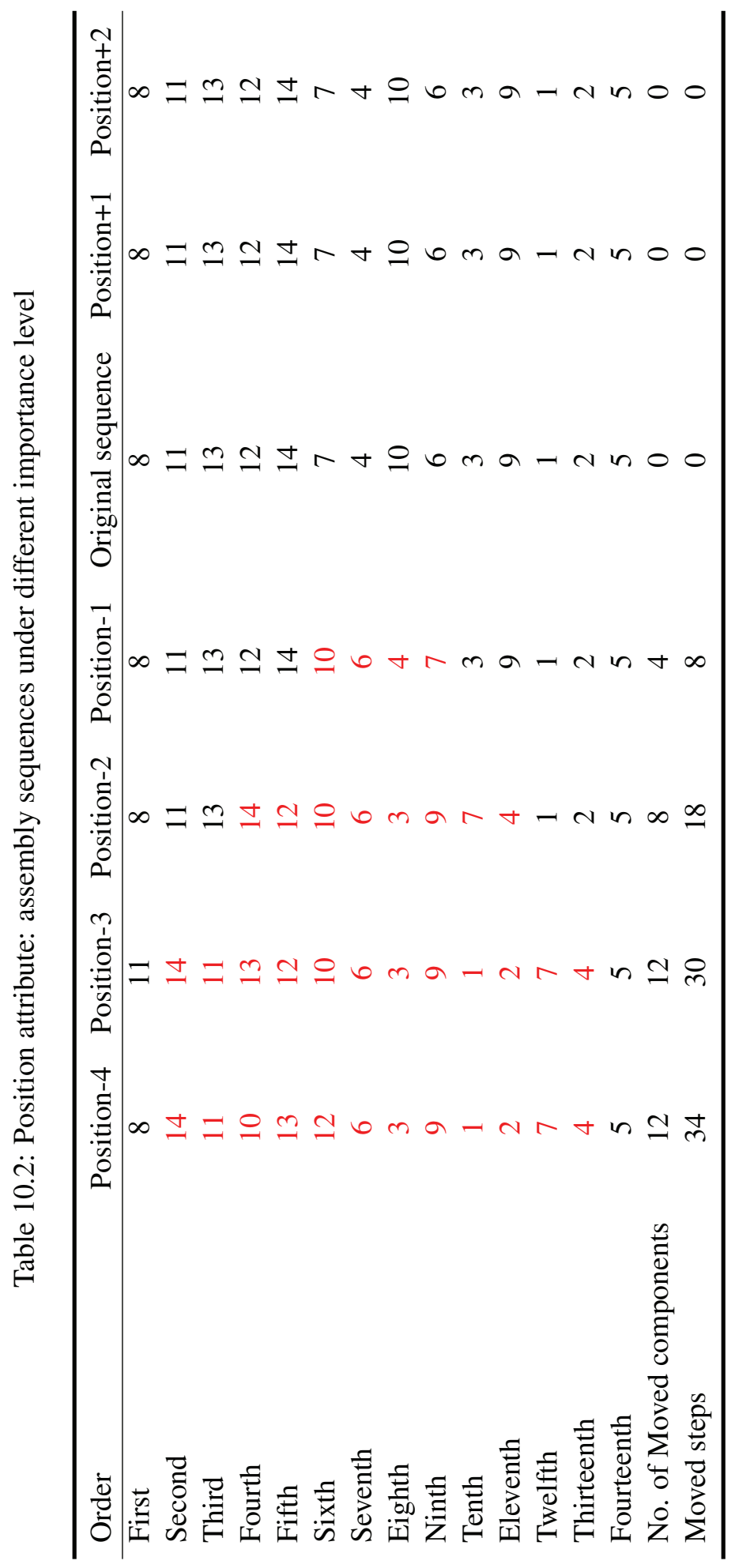


150 Automatic generation of assembly sequence for the planning of outfitting processes in shipbuilding

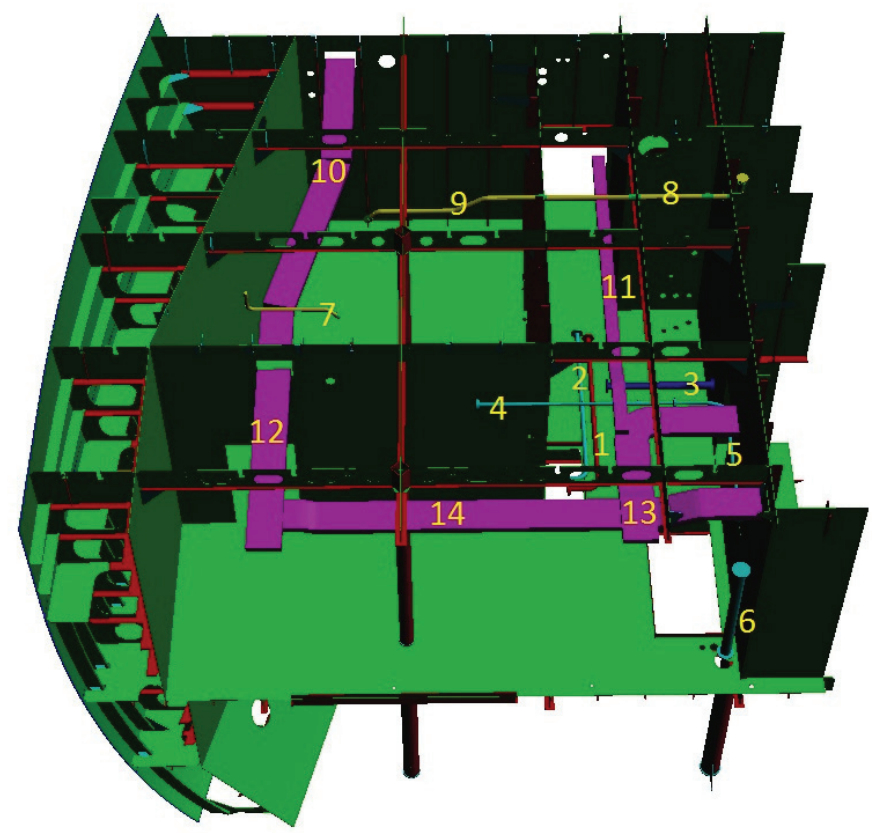

Figure 10.2: A test case with 14 components

It also can be found, see figure 10.3, that a further increase of its importance level to one or two levels higher (position+1 and position+2) does not bring any further change. The assembly sequences with these two levels remain the same.

In conclusion, when the importance level of position attribute is decreased, the model runs the risk to generate unreasonable assembly sequences.

\section{Material attribute}

The assembly sequence is not sensitive to the material attribute. In reality, apart from a few number plastic, bronze pipes or spiro-ducts, most of outfitting components are made of steel. This indicates that most of the components share the same material ranking. From figure 10.4 it is clear that the increase or decrease of its importance level hardly brings great changes to the final sequence. 


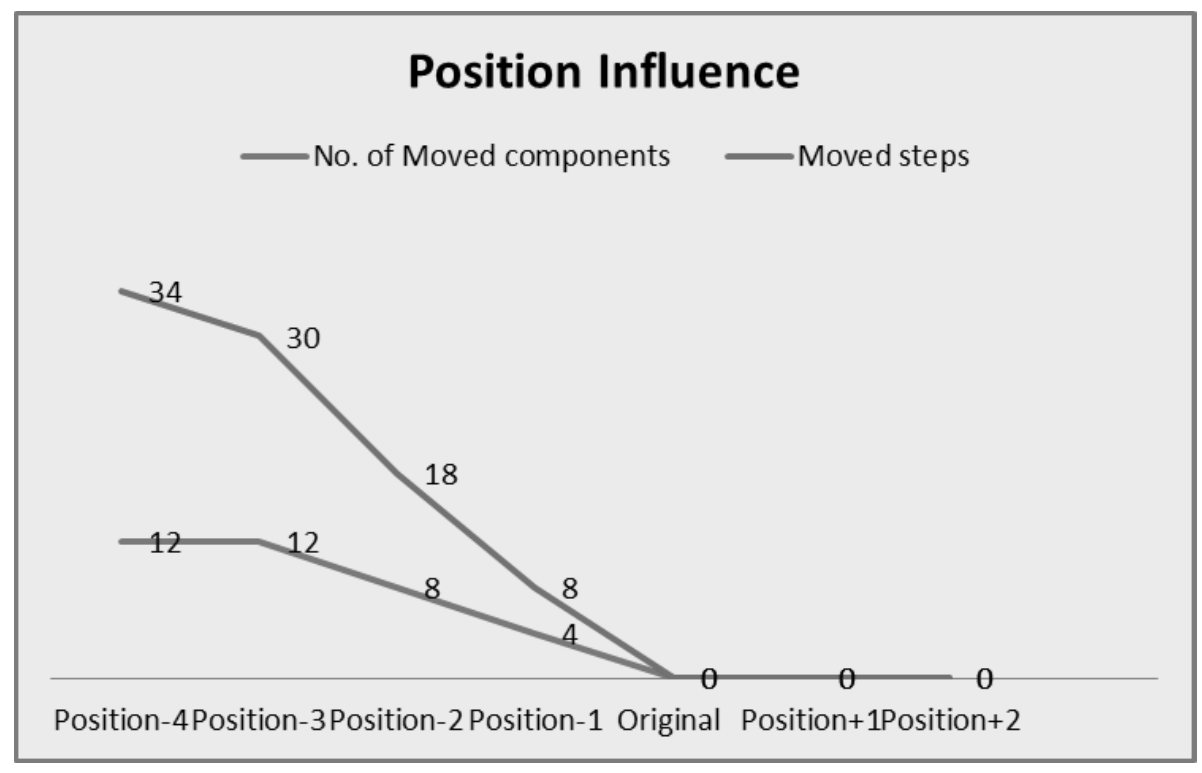

Figure 10.3: Position influence

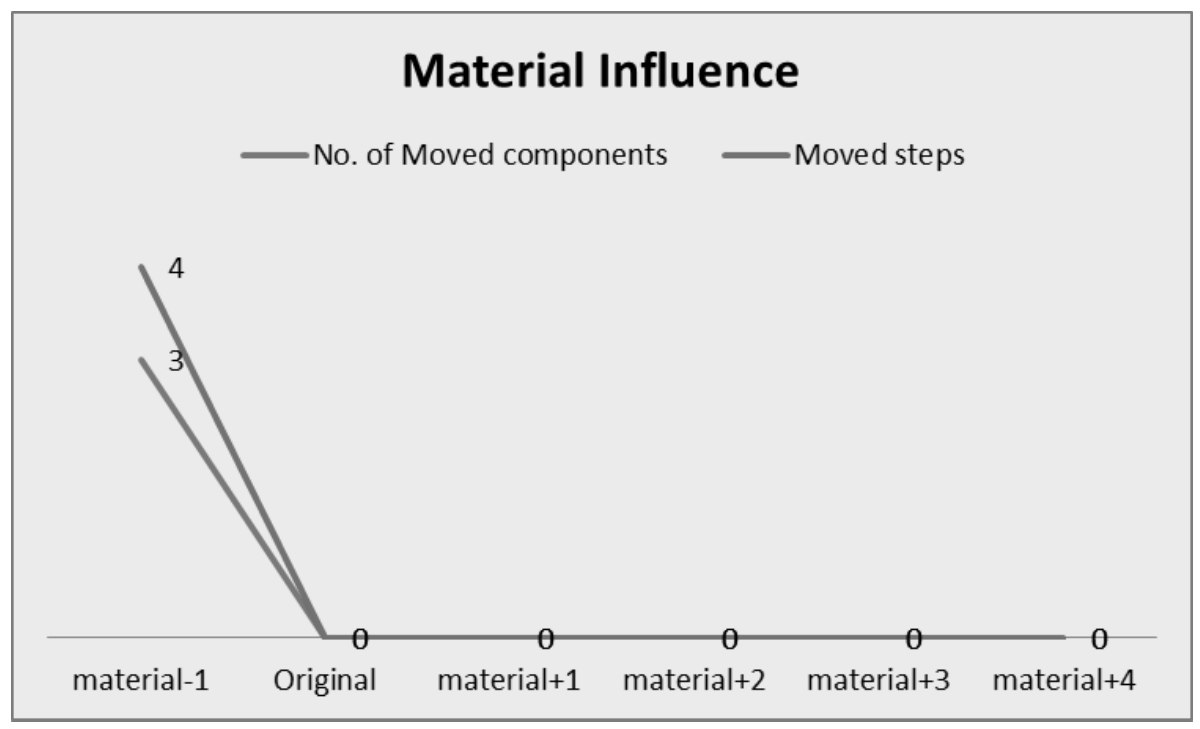

Figure 10.4: Material influence 
152 Automatic generation of assembly sequence for the planning of outfitting processes in shipbuilding

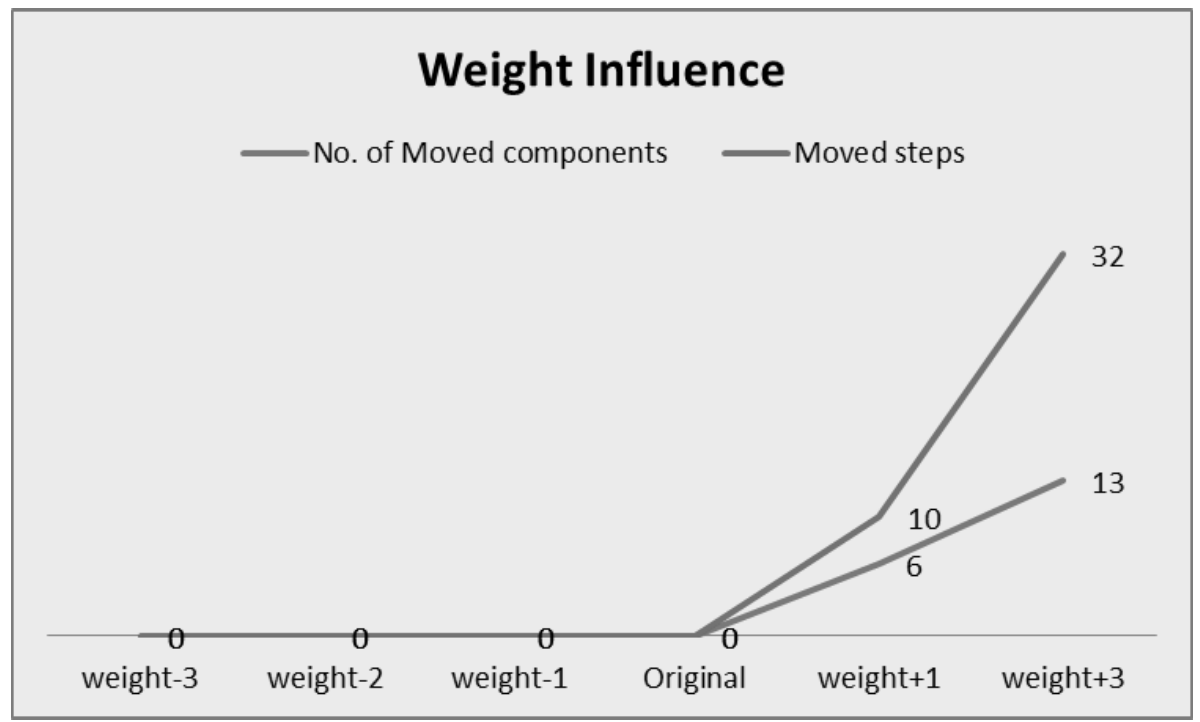

Figure 10.5: Weight influence

\section{Weight attribute}

The assembly sequence becomes sensitive to the weight attribute when its importance level is increased. In figure 10.5, the increase of its importance level (weight+1 and weight +2 ) induces important changes in the assembly sequence.

\section{Size attribute}

The assembly sequence is slightly less sensitive to the size attribute than to the weight attribute when its importance level becomes higher. When both size and weight's importance are three levels higher, i.e. size +3 and weight +3 , it can be seen in figure 10.6 that number of deviations due to the weight influence is higher than that of the size.

\section{Penetration attribute}

The assembly sequence is less sensitive to the penetration attribute than to the position attribute. It is because in real life, only a small percentage of spools is penetrating and goes through steel structures in a section. Figure 10.7 shows that in general, the deviation scale under the influence of penetration attribute is smaller than that of position.

The conclusion of the above analysis is drawn and presented in table 10.3. The assembly sequence becomes sensitive to weight and size attributes when their im- 


\section{Size Influence}

No. of Moved components $\quad$ Moved steps

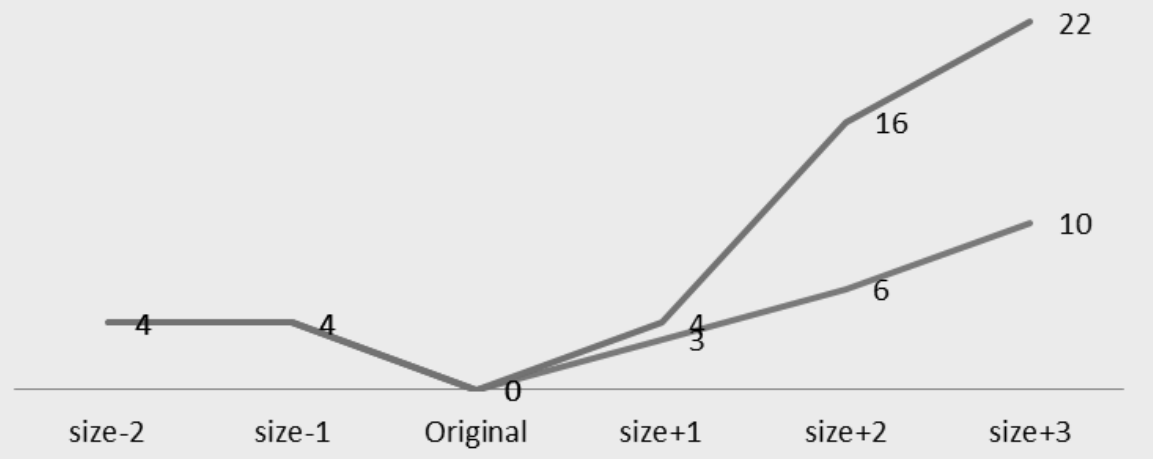

Figure 10.6: Size influence

\section{Penetration Influence}

No. of Moved components $\quad$ Moved steps

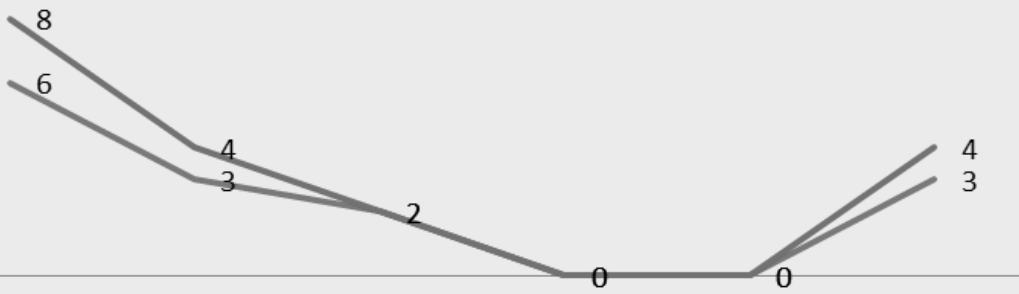

Penetration-4 Penetration-3 Penetration-2 penetration-1 Original penetration +1

Figure 10.7: Penetration influence 
154 Automatic generation of assembly sequence for the planning of outfitting processes in shipbuilding

portance levels are increased; it also becomes sensitive to position and penetration attributes when their importance levels are decreased; it is not sensitive to the material attribute. Thus, an increase of the current importance levels of position and penetration, which also implies a decrease of the current importance levels of material, size and weight, may result in a better assembly sequence that can avoid the situation shown in figure 9.13.

Table 10.3: Conclusion of the sensitivity analysis

\begin{tabular}{lccccc}
\hline & Position & Material & Weight & Size & Penetration \\
\hline Sensitive when its importance level is increased & & & +++ & +++ & + \\
Sensitive when its importance level is decreased & +++ & & & + & +++ \\
Not sensitive & & +++ & & & \\
\hline
\end{tabular}

\subsection{The sensitivity to the minimum work distance}

The minimum work distance was set to a constant, used to determine whether the distance between two outfitting components is sufficient to allow them to be assembled in parallel. This parameter influences the number of finish-start relationships of component assembly and the total duration time. On the work site, the minimum work distance is claimed to be crucial, but a precise value is not provided by the workers. Field study indicates it should be in the range of 2-3 m. Based on the analysis from the ergonomics aspect described in chapter 6.1.6, the minimum work distance was set as $2.5 \mathrm{~m}$ in the research. This section is devoted to the analysis of how the minimum distance influences the duration time and the maximum number of components that can be mounted simultaneously.

Here, a test case with 10 components in figure 10.8 is used to illustrate the analysis. The distance between any two of the components is calculated as shown in figure 10.9 and ranges from 0 to $5319.8 \mathrm{~mm}$. The assembly sequences are generated while varying the minimum distance from $2000 \mathrm{~mm}$ to $3000 \mathrm{~mm}$. Following the same procedure, test cases with different number of components have been also been applied.

Table 10.4 shows that the assembly sequence has shorter duration time when reducing the minimum work distance due to the fact that more components can be assembled in parallel and fewer dependencies are related.

For different test cases and for various minimum work distances, the maximum number of components that can be mounted in parallel has also been listed in table 10.5. It shows that as the minimum work distance increases, the maximum number of components mounted in parallel decreases. When the minimum distance was set at the maximum value of $3000 \mathrm{~mm}$, the duration time reached the highest and the maximum number of components mounted in parallel, on the other hand, drops to 


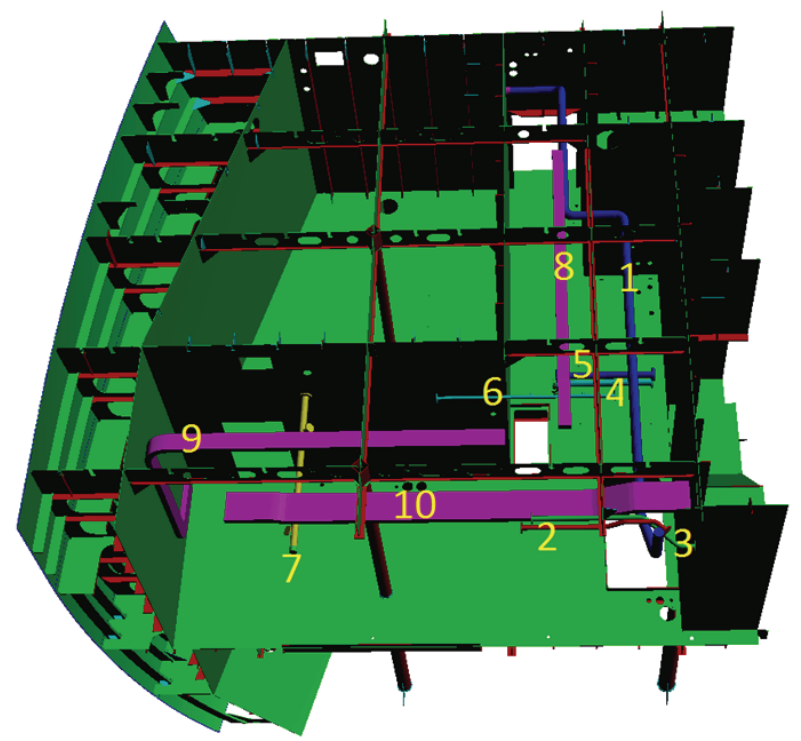

Figure 10.8: A test case with 10 components

\begin{tabular}{|l|l|l|l|l|l|l|l|l|l|l|l|}
\hline & 1 & 2 & 3 & 4 & 5 & 6 & 7 & 8 & 9 & 10 \\
\hline 1 & 0 & 315 & 155 & 450 & 220 & 346 & 5320 & 189 & 2108 & 231 \\
\hline 2 & 315 & 0 & 160 & 3109 & 3073 & 2410 & 3618 & 1701 & 1699 & 477 \\
\hline 3 & 155 & 160 & 0 & 2953 & 2914 & 2250 & 3777 & 1543 & 1574 & 310 \\
\hline 4 & 450 & 3109 & 2953 & 0 & 230 & 874 & 4201 & 621 & 1692 & 2619 \\
\hline 5 & 220 & 3073 & 2914 & 230 & 0 & 737 & 4188 & 394 & 1637 & 2589 \\
\hline 6 & 346 & 2410 & 2250 & 874 & 737 & 0 & 2137 & 215 & 737 & 1940 \\
\hline 7 & 5320 & 3618 & 3777 & 4201 & 4188 & 2137 & 0 & 4197 & 290 & 230 \\
\hline 8 & 189 & 1701 & 1543 & 621 & 394 & 215 & 4197 & 0 & 1007 & 1271 \\
\hline 9 & 2108 & 1699 & 1574 & 1692 & 1637 & 737 & 290 & 1007 & 0 & 1208 \\
\hline 10 & 231 & 477 & 310 & 2619 & 2589 & 1940 & 230 & 1271 & 1208 & 0 \\
\hline
\end{tabular}

Figure 10.9: Distance calculation between any two components in the 10-component test case

the lowest value.

The percentages of the increase of duration time and of the decrease of the maximum number of components mounted in parallel are also shown in tables 10.4 and 10.5. The average percentage of duration time increase is $20 \%$ and the average percentage decrease of the maximum number is $32 \%$ for an increase of the minimum work distance from $2000 \mathrm{~mm}$ to $3000 \mathrm{~mm}$. Having small employees would seem to pay off. However, this strong influence is tied to a substantial increase in resources since at a smaller work distance, more teams can work simultaneously. Allowing for this effect would greatly diminish the sensitivity.

The assembly process is sensitive to the total duration time and the maximum number of components that could be installed in parallel. To set the minimum work distance at $2500 \mathrm{~mm}$ in the research is mainly based on the experience, and it still 


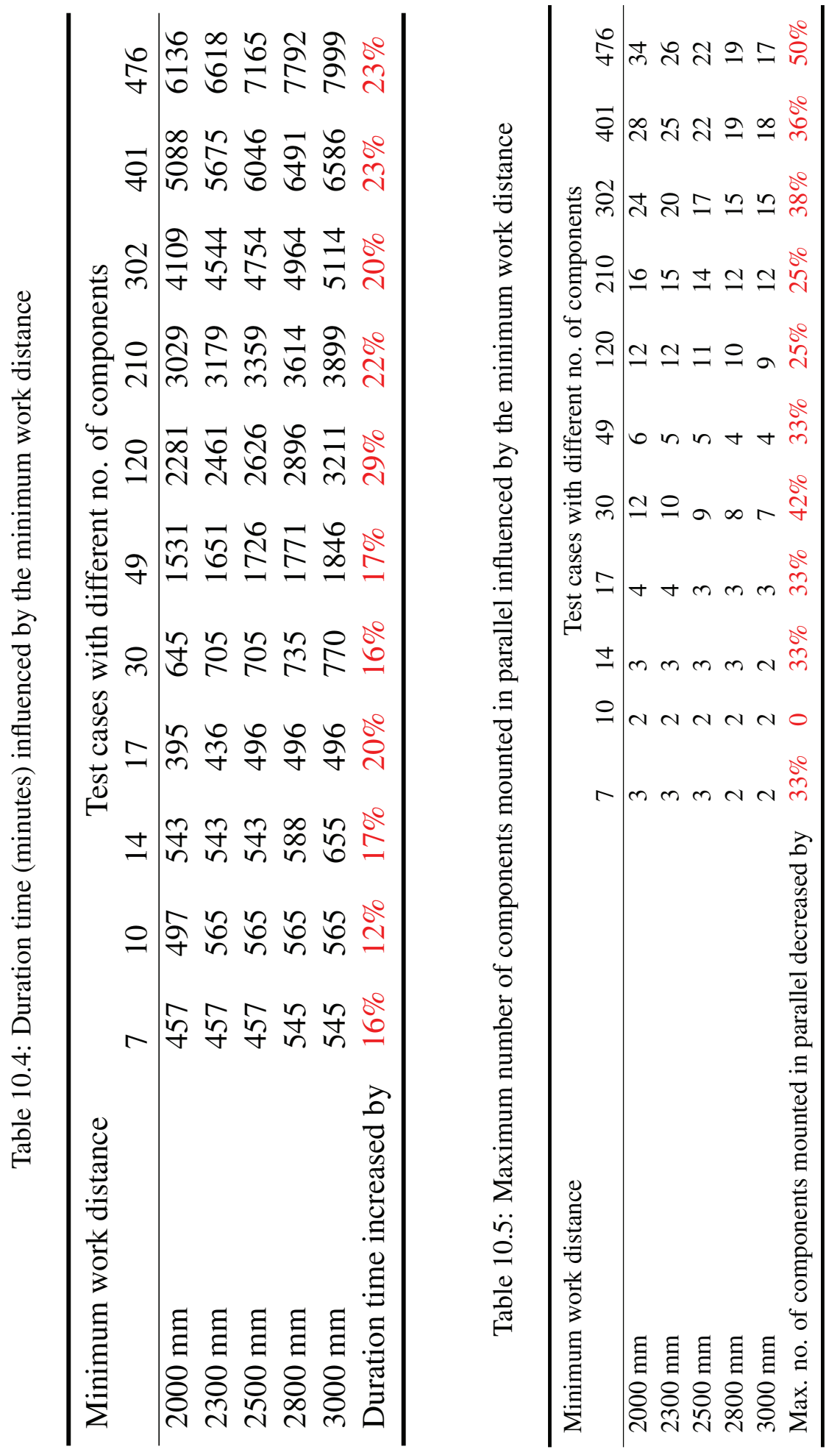


requires the comparison of duration time with reliable throughput times in real life to validate it properly. It is believed that in the future, further information-collection in the field may improve the accuracy of minimum work distance estimation.

\subsection{The analysis of two versions of assembly sequences}

As we have discussed in chapter 9.1.2, two versions of the assembly sequences, Downward version and Upward version, can be obtained from the model. To select the better one mainly depends on which version has shorter duration time. In this section, we investigate how the duration time is influenced by the two versions.

In table 10.6, test cases with random numbers of components chosen from steel section 1-4 are presented. The duration time resulting from the two versions have been calculated.

Table 10.6: Duration time comparison between two versions of assembly sequences

\begin{tabular}{|c|c|c|c|c|c|c|}
\hline & \multicolumn{6}{|c|}{ Steel section 1} \\
\hline Test cases with different no. of components & 49 & 120 & 210 & 302 & 401 & 522 \\
\hline Upward (hour) & 31 & 44 & 56 & 78 & 105 & 125 \\
\hline Downward(hour) & 29 & 44 & 56 & 79 & 101 & 118 \\
\hline \multirow[t]{2}{*}{ Increased/Decreased by } & $-6,50 \%$ & 0 & 0 & $1,30 \%$ & $-3,80 \%$ & $-5,60 \%$ \\
\hline & \multicolumn{6}{|c|}{ Steel section 2} \\
\hline Test cases with different no. of components & 30 & 81 & 127 & 232 & 415 & 462 \\
\hline Upward (hour) & 9 & 30 & 40 & 73 & 120 & 124 \\
\hline Downward(hour) & 9 & 29 & 42 & 65 & 90 & 98 \\
\hline \multirow[t]{2}{*}{ Increased/Decreased by } & 0 & $-3,30 \%$ & $5 \%$ & $11 \%$ & $-25 \%$ & $-21 \%$ \\
\hline & \multicolumn{6}{|c|}{ Steel section 3} \\
\hline Test cases with different no. of components & 45 & 132 & 237 & 279 & 321 & 436 \\
\hline Upward (hour) & 16 & 38 & 84 & 87 & 93 & 126 \\
\hline Downward(hour) & 16 & 40 & 68 & 82 & 89 & 109 \\
\hline \multirow[t]{2}{*}{ Increased/Decreased by } & 0 & $5,30 \%$ & $-19 \%$ & $-5,70 \%$ & $-4,30 \%$ & $-13,50 \%$ \\
\hline & \multicolumn{6}{|c|}{ Steel section 4} \\
\hline Test cases with different no. of components & 10 & 59 & 86 & 131 & 202 & 222 \\
\hline Upward (hour) & 16 & 34 & 38 & 48 & 52 & 78 \\
\hline Downward(hour) & 16 & 38 & 42 & 49 & 69 & 98 \\
\hline Increased/Decreased by & 0 & $11,80 \%$ & $10,50 \%$ & $21 \%$ & $32,70 \%$ & $25,60 \%$ \\
\hline
\end{tabular}

In table 10.6, it can be found that only in the test cases for steel section 4 , the duration time of the Upward version is always less than that of the Downward version. In the test cases for the other 3 sections, the percentages can take both positive and negative values. To explain this phenomenon, we take a further look at the attributes of these four sections.

Steel section 4 is the wheel house section, which implies that more components (typically cable trays) are located near the floor area. It would be easier to assemble 
158 Automatic generation of assembly sequence for the planning of outfitting processes in shipbuilding

them when the section is in the upright position. It corresponds to the Upward version in which the interference detection takes place along $+Z$ (from bottom of a ship to top of a ship) direction. The assembly sequence obtained from the Upward version prefers sections to be upright because the translation direction is the same as sections' position. Sequences resulting from the Downward version fit in the situation when sections are turned upside down. In the test cases for section 4, the Upward versions have lower duration time. Thus, steel section 4 is preferred to be pre-outfitted in the upright position.

Steel section 1 is an engine room workshop section and section 2 and 3 are engine room sections. Components are fairly equally distributed between locations close to the ceilings or floors. Some are easier to be assembled when the sections are turned upside down and some require the section to be in the upright position. Thus, the comparison of the duration time for two versions of the assembly sequences may provide indications of the preferred section position when to pre-outfit it.

\subsection{Summary}

The sensitivity analysis of the inputs of the model helps to gain insight in how a change of inputs influences the assembly sequences. Based on the discussions in this chapter, the following conclusions are summarized:

- Assembly sequences are sensitive to changes in the weighting of various attributes. An increase in the weighting of weight and size and a decrease in the weighting of position and penetration result in the biggest sensitivity. The sequence is not sensitive to the material attribute. The derivation of reasonable weighting coefficients hinges on the judgment of skilled employees so that the contribution of these attributes to the assembly sequences can be appropriately represented.

- The duration time of assembly sequence is rather sensitive to the minimum work distance, provided unlimited resources are available. This is because its value decides the number of components that could be assembled in parallel under the same height level. To set the proper value for the minimum distance requires further field study.

- Two versions of assembly sequence generation (upward and downward) result in different duration times for the same test case. The one having the lower duration time may be regarded as the more suitable sequence for the pre-outfitting of a certain section. This is not only because the lower duration time implies lower cost but also because it indicates the better position, either upright or upside down to carry out the pre-outfitting work of the section. 


\section{Chapter 11}

\section{Conclusions and Recommendations}

This research presents the possibilities to improve outfitting processes by means of integrating the assembly of different type of components, expressed by an automatic generation system of assembly sequences. To reduce the coordination efforts on the work site due to the frequent interferences among yard and different subcontractors, the application of a sequence generation system contributes to the clarification of dependencies to be used in the planning system. The output of this research project is not the development of another scheduling tool that commits all kinds of resources between a variety of possible tasks, but the determination of the technical dependencies among different assembly tasks that helps to optimize both the planning and production processes. The assembly sequence generation system contains three fundamentally automated processes: assembly knowledge representation, data acquisition and preparation and sequence generation. Based on the functionalities of these processes, the conclusions of this research are presented in section 11.1. Section 11.2 gives recommendations for future research.

\subsection{Conclusions}

The research presents an automatic generation system of assembly sequences, which inputs the engineering information and captures the assembly knowledge to automate and optimize the process planning of assembly in outfitting. This section describes the conclusions by checking the research objectives (as defined in section 1.2) against the proposed sequence generation model and the results obtained in this research.

\section{Capture the assembly knowledge in terms of technical aspects}

Under the assumption that the resources to carry out the assembly work are available, the model only takes the technical aspects of assembly knowledge into account. It is 
160 Automatic generation of assembly sequence for the planning of outfitting processes in shipbuilding

capable of selecting the most relevant geometrical attributes of outfitting components and expressing them in a mathematical way. The introduction of the 'interference matrix', adopted from the mechanical industry, has been crucial to the detection of the spatial relationship of components. The concept of 'weighting coefficients' is applied to determine the role of different attributes in deciding the mounting orders. Therefore the presented model certainly has captured important parts of the assembly knowledge, which for a long time was implicit and remained as expertise only possessed by senior workers. However, it should not be forgotten that this knowledge capture has not addressed resource considerations.

\section{Fill the gap between engineering and planning}

The model has taken the detailed engineering (3D CAD) model as input and established a largely automated data-preparation model to select, reorganize and manipulate the data. It is not only able to read the data extracted from the engineering model but provides a platform allowing original data to be modified and extra data to be added in. It has succeeded, with certain limitations mainly imposed by the incompleteness of 3D CAD ship models, to bridge the gap between engineering and production by a mechanism other than human intellectual labor. Thus, it clearly helps to derive a planning from design and engineering data with less involvement of man hours, paper drawings and specifications while keeping the same accuracy of information.

\section{Provide dependencies of assembly work}

With the fulfillment of all technical requirements, the model presents reasonable dependencies within assembly work. The application of the 'Basket Done Method' managed to incorporate the effect of the minimum work distance and only allowed those components to be assembled in parallel for which the distance is sufficient. Thus, apart from the geometrical attributes of the component itself, the space accessibility is also taken into account. The overview of dependencies helps to optimize the decision-making processes for planners when they are confronted with different sorts of resources for all components in a section.

\section{Present a starting point for the simulation of outfitting processes}

The development of the model successfully retrieved and interpreted the outfitting components' data and reorganized them in a way that can be accessed for simulation purpose. Moreover, their dependencies expressed in the form of a sequence are vital input to allow the detailed simulation of outfitting processes. Meanwhile, the animation of the assembly sequence, combined with an e-viewer of the 3D CAD model, shows promises for further automatic assembly instructions or even operations on the work site. Their integration plus simulation may make available a powerful tool to guide workers to carry out daily outfitting assembly work in the shipbuilding industry. 
The automatic generation system of assembly sequences was implemented and evaluated using real test cases, two of which are main-engine sections and possessed a most complicated configuration of assembly components. The validation based on qualitative interviews and quantitative analysis showed that the generated assembly sequences were realistic, although certainly not yet perfect.

\subsection{Recommendations}

The development of automatic assembly sequence generation in the field of outfitting processes in shipbuilding is still at an early stage. The realization of a fully automated, robust, trustworthy and adaptive system still relies on further research and thorough investigation. The most important recommendations are presented here.

- Apart from being able to detect the spatial relationships of spools along the $\mathrm{Z}$ direction (bottom-top of a ship), the model should also be expanded to be able to find them along $\mathrm{X}$ direction(stern to bow) and $\mathrm{Y}$ direction (portside to starboard). It would help to make sure that components closest to the steel structure boundaries, like walls, ceilings, and floors, would be assembled first.

In order to find such relationships, the distance between spools and steel walls and ceilings should be known, either by calculation or by estimation. This requires the use of steel structure 3D coordinates. Indeed, since the details of the 3D structure are always available, a more advanced method of geometrical analysis may lead to a superior interference matrix that also takes the possibilities of maneuvering components into account. However, it is by no means certain that this much more complex method would also lead to better practical usability of the method.

- Develop a way to incorporate the 'mounting stage' into the model.

Some components can only be installed when the steel section is open, for example pipes located in the double bottom section, because once the section is closed, it is almost impossible to get these spools into the steel structure and assemble them. Another kind of spools, which cross more than one section, can only be installed after these sections are joined together on the slipway. When the mounting stage is included, it can be filtered for component availability. This could in principle be derived from a sufficiently detailed procurement schedule.

Again, the availability of a complete 3D CAD model offers many, fairly complex opportunities. One could combine the CAD model with a large-scale ship structure assembly sequence. Geometrical analysis routines could assess the transportation routes for all components given the ship assembly status. It could even derive maneuvering space. Such analysis would help to analyze 
162 Automatic generation of assembly sequence for the planning of outfitting processes in shipbuilding

automatic divisions between pre-outfit and outfit and provide data to assess transportation and handling times. Our research has shown that this approach can be fruitful in deriving realistic sequences.

- Implement the 'roadmap' which may lead to the possible way to automatically generate a more realistic equipment installation sequence. The core of the 'roadmap' is to complete the equipment data preparation model. It includes:

- to assign the start and finish date of pre-outfitting and hull erection for each section;

- to couple each compartment with its sections and specify what equipment is in which sections;

- to detect the open-sides of a section which provides access for the equipment;

- to filter the equipment list with respect to the procurement planning to ensure that a component is delivered in time for the intended mounting date;

- to collect the geometrical data of equipment from 3D model, including its size, weight, connection points and exact position.

- Estimate the duration time of assembling spools more accurately. Because of the lack of any information about pipe, HVAC and cable tray supports in the engineering model, their number and mounting time were obtained from the field observation. In reality, the manufacturing time of pipe supports in the warehouse varies, depending on their type and position. Thus, more relevant information needs to be collected in real-life projects.

A videotape on the jobsite is suggested to obtain a record of actual assembly times. It is especially important to automate the calculation of mounting time for pieces of equipment. A detailed questionnaire and subsequent statistical analysis would provide a feasible approach.

- Combine the method with a suitable schematic method of the onboard systems. This would allow topological information to be used in order to decide the mounting order of components that are connected to each other, e.g., a number of spools forming one pipe. 


\section{A \\ The derivation of relative importance of the five physical attributes}

In order to obtain the relative importance of the five physical attributes, the interviews with foremen and workers (together 4 persons) were conducted. The interviews and the calculation of the relative importance have been described here.

First, the interviewers gave the explanations of these five physical attributes according to their customs:

- Position-where the outfitting component is located in a ship;

- Material- what material the outfitting component is made of;

- Weight-how heavy the outfitting component is;

- Size- how big the outfitting component is;

- Penetration- whether the outfitting component go through a piece of watertight steel structure (wall, bulkhead, etc.)

Then, the following example was presented to them. Suppose we have two fruits, apple and banana. I would like to ask you, which fruit you like better than the other and how much you like it in comparison with the other. Let us make a relative scale to measure how much you like the fruit on the left (Apple) compared to the fruit on the right (Banana), see A.1.

If you like the apple better than banana, you thick a mark between number 1 and 9 on left side, while if you favor banana more than apple, then you mark on the right side. For instance I strongly favor banana to apple then I give mark like A.2. 
164 Automatic generation of assembly sequence for the planning of outfitting processes in shipbuilding

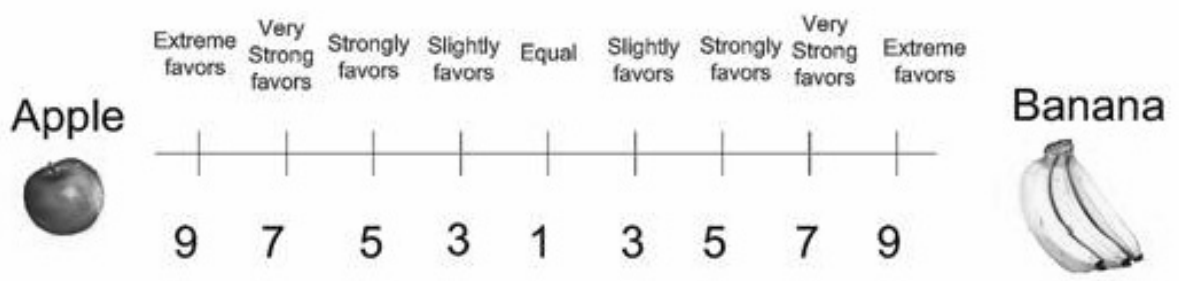

Figure A.1: Preference level between apple and banana (reproduced from Hart (Teknomo, 2006))

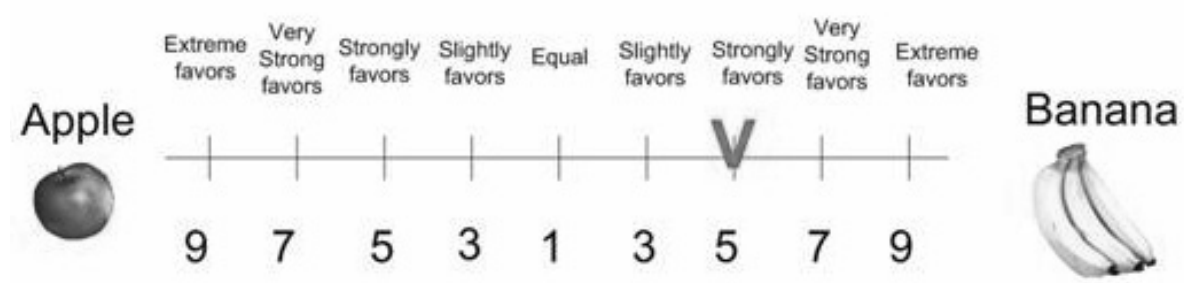

Figure A.2: Mark your preference (reproduced from Hart (Teknomo, 2006))

In the same principle, concerning the importance level of components physical attributes, we can make the same comparison. There are five attributes-Position, Material, Size, Weight and Penetrationin the determination of the assembly sequence.

Now I would like to ask you, which attribute is more important than the other and how much more important you think it is. The comparisons between two attributes are listed as follows:

Position: 975313579 Material

Position: 975313579 Weight

Position: 975313579 Size

Position: 975313579 Penetration

Material: 975313579 Weight

Material: 975313579 Size

Material: 975313579 Penetration

Weight: 975313579 Size 
Weight: 975313579 Penetration

\section{Size : 975313579 Penetration}

The results from the interviewers are listed in table A.1. If the judgment value is on the left side of 1 , we put the actual judgment value; if the judgment value is on the right side of 1 , we put the reciprocal value. The numeric values from column 'Average' are the final judgment value after comparing four answers.

Table A.1: The pairwise comparison derived from the interviewers

\begin{tabular}{llllll}
\hline & Answer1 & Answer2 & Answer3 & Answer4 & Average \\
\hline Position-Material & 9 & 9 & 7 & 9 & 9 \\
Position-Weight & 7 & 7 & 7 & 7 & 7 \\
Position-Size & 5 & 5 & 5 & 7 & 5 \\
Position-Penetration & 1 & $1 / 3$ & 1 & 1 & 1 \\
Material-Weight & $1 / 5$ & $1 / 3$ & $1 / 7$ & $1 / 5$ & $1 / 5$ \\
Material-Size & $1 / 5$ & $1 / 3$ & $1 / 3$ & 1 & $1 / 3$ \\
Material-Penetration & $1 / 7$ & $1 / 7$ & $1 / 7$ & $1 / 9$ & $1 / 7$ \\
Weight-Size & 1 & 1 & $1 / 3$ & 1 & 1 \\
Weight-Penetration & $1 / 5$ & $1 / 3$ & $1 / 3$ & $1 / 3$ & $1 / 3$ \\
Size-Penetration & $1 / 3$ & $1 / 5$ & $1 / 5$ & $1 / 7$ & $1 / 5$ \\
\hline
\end{tabular}

From the above comparisons, we can make a pairwise comparison matrix as follows:

$\left[\begin{array}{cccccc}\text { Attribute } & \text { Position } & \text { Material } & \text { Weight } & \text { Size } & \text { Penetration } \\ \text { Position } & 1 & 9 & 7 & 5 & 1 \\ \text { Material } & & 1 & 1 / 5 & 1 / 3 & 1 / 7 \\ \text { Weight } & & & 1 & 1 & 1 / 3 \\ \text { Size } & & & & 1 & 1 / 5 \\ \text { Penetration } & & & & & 1\end{array}\right]$

The diagonal elements of the matrix are always 1 and we only need to fill up the upper triangular matrix.

From the above pairwise comparison matrix, its normalized principal Eigen vector can be calculated and is displayed as follows:

$$
W=\left[\begin{array}{l}
0.434 \\
0.035 \\
0.104 \\
0.087 \\
0.340
\end{array}\right]
$$


166 Automatic generation of assembly sequence for the planning of outfitting processes in shipbuilding

The normalized principal Eigen vector is also called priority vector. Since it is normalized, the sum of all elements in priority vector is 1 . The priority vector shows relative weights among the five attributes that we compare. In our example above, position is $43.4 \%$, material is $3.5 \%$, weight (mass) is $10.4 \%$, size is $8.7 \%$ and penetration is $34 \%$.

Aside from the relative weight, we can also check the consistency of the interviewers' answers. To do that, we need what is called Principal Eigen value $\lambda_{\max }$. Saaty (Saaty, 1980) proved that for consistent reciprocal matrix, the largest Eigen value is equal to the size of comparison matrix , or $\lambda_{\max }=\mathrm{n}$. Then he gave a measure of consistency, called Consistency Index as deviation or degree of consistency using the following formula:

$$
C I=\frac{\lambda_{\max }-n}{n-1}
$$

Thus, in our previous example, we have $\lambda_{\max }=5.19179$ and the size of comparison matrix is $\mathrm{n}=5$, thus the consistency index is:

$$
C I=\frac{\lambda_{\max }-n}{n-1}=\frac{5.19179-5}{4}=0.0479
$$

If the value of $\mathrm{CI}$ is smaller or equal to $10 \%$, the inconsistency is acceptable because a human is not always consistent. If it is greater than $10 \%$, we need to revise the subjective judgments. In our case, the CI is $4.79 \%$, less than $10 \%$. Thus, the interviewers' subjective evaluation about the importance of five physical attributes is consistent. 


\section{B}

\section{Preliminary estimation of mounting time}

The preliminary estimation of mounting time is based on field studies in shipyards. The means to carry out the field studies is the combination of observations and interviews with foremen and workers. During these field studies, the outfitting assembly activities were recorded and their durations were obtained. For different types of outfitting components, the way to estimate its mounting time is reported as follows:

- Preliminary estimation of mounting time of pipe spools.

The observation had been carried out from July, 2008 to April, 2009, one day per week. The result is shown in table B.1

In conclusion, for a pipe spool, the mounting time is calculated in this way: 30 minutes for preparation; 45 minutes for transporting it by crane if the weight of a pipe spool is more than $50 \mathrm{~kg}$ and if the weight is less than $50 \mathrm{~kg}$, there is no such time; 30 minutes for each support including measuring, making and welding it. If the length of a pipe spool is less than $3000 \mathrm{~mm}$, at least one support is needed.

Mounting time $=30+45$ (or 0$)+30 *($ length $/ 3000) .3000 \mathrm{~mm}$ is the span between

Table B.1: Estimate mounting time of pipe spools

\begin{tabular}{llc}
\hline Item & Content & Time on average (minute) \\
\hline Prepare documents and tools & Read 3D and 2D drawings & 10 \\
& Think and make the decision which is the next spool to be installed & 10 \\
& Search the spool in a pipe tray & 5 \\
& Negotiate with the current user of a crane & 20 \\
& Wait for the crane & \\
& Transport the pipe spool from the pipe tray to a steel section and place the pipe spool on its position \\
\hline Transport a pipe spool & Figure out the position of this support & \\
& Measure and write down the distance from the center of the pipe spool to its nearby steel structure & \\
& Walk to a workshop and make the support & 15 \\
& Pick up bolts and nuts in a storehouse and walk back to the steel section & 5 \\
& Weld the support on the steel structure and put the pipe spool on the support & 5 \\
\hline
\end{tabular}


168 Automatic generation of assembly sequence for the planning of outfitting processes in shipbuilding

Table B.2: Estimate mounting time of cable trays

\begin{tabular}{llc}
\hline Item & Content & Time on average (minute) \\
\hline Make a pair of supports & Read drawings & 3 \\
& Find the supports' position & 3 \\
& Calculate their height & 1 \\
& Cut a piece of angle bar according to the measured height & 1 \\
& Weld the supports on the steel structure & 2 \\
\hline Align the current cable tray & Put the current cable tray on the welded supports & 2 \\
& Align the current cable tray with the previous ones & 3 \\
\hline Weld the cable tray & Weld the cable tray on a pair of its supports & 2 \\
\hline
\end{tabular}

every support.

- Preliminary estimation of mounting time of HVAC ducts

The observation of HVAC ducts installation started almost half a year later than that of pipe spools, which was from February, 2009 to November, 2009.

For HVAC ducts, the installation of couplings is the most time-consuming part due to the strict requirements during their complicated installation procedures. According to the observation which was carried out in 4 days consecutively in September, 2009, 11 pieces of Spiro-duct were installed in an accommodation steel section. These ducts contain 403 couplings. It took four workers 4 days to mount them, which means that 128 man-hours were spent. Thus, $128 * 60 / 403=19$ minutes per coupling.

- Preliminary estimation of mounting time of cable trays. The observation of cable trays installation was carried simultaneously with that of HVAC ducts. The result is shown in table B.2.

In conclusion, for a piece of cable tray, the mounting time is calculated in this way: 10 minutes for making a pair of supports; 5 minutes for aligning the current cable tray with previous or surrounding ones; 2 minutes for welding them on the supports. If the length of a piece of cable tray is less than 3000 $\mathrm{mm}$, at least one pair of supports is needed.

Mounting time $=10 *($ length $/ 3000)+5+2 *($ length/3000). $3000 \mathrm{~mm}$ is the span of every pair of support. 


\section{C}

\section{UML class diagram}

During the data collection and preparation stage, the UML class diagram in figure C.1 is applied to express the operations and attributes of different data classes and to show their interactions. In the class diagram, these classes are represented with boxes which contain three parts (Eriksson, 2004):

- The upper part of holds the name of the class, like 'TSpool' in figure C.1 is the name of spool class;

- The middle part contains the attributes of the class, for example, the spool class has the attributes of name, pieces, mounting time, etc.;

- The bottom part gives the methods or operations that can be performed on the class, for example, the operation of comparing weight can be done to each spool.

The interactions between classes are expressed by links, which is called 'association'. In figure C.1, the association between class 'TEquipments' and class 'TBoundingBox' means that each piece of equipment has a unique bounding box and bounding box expresses one or many pieces of equipment. 
170 Automatic generation of assembly sequence for the planning of outfitting processes in shipbuilding

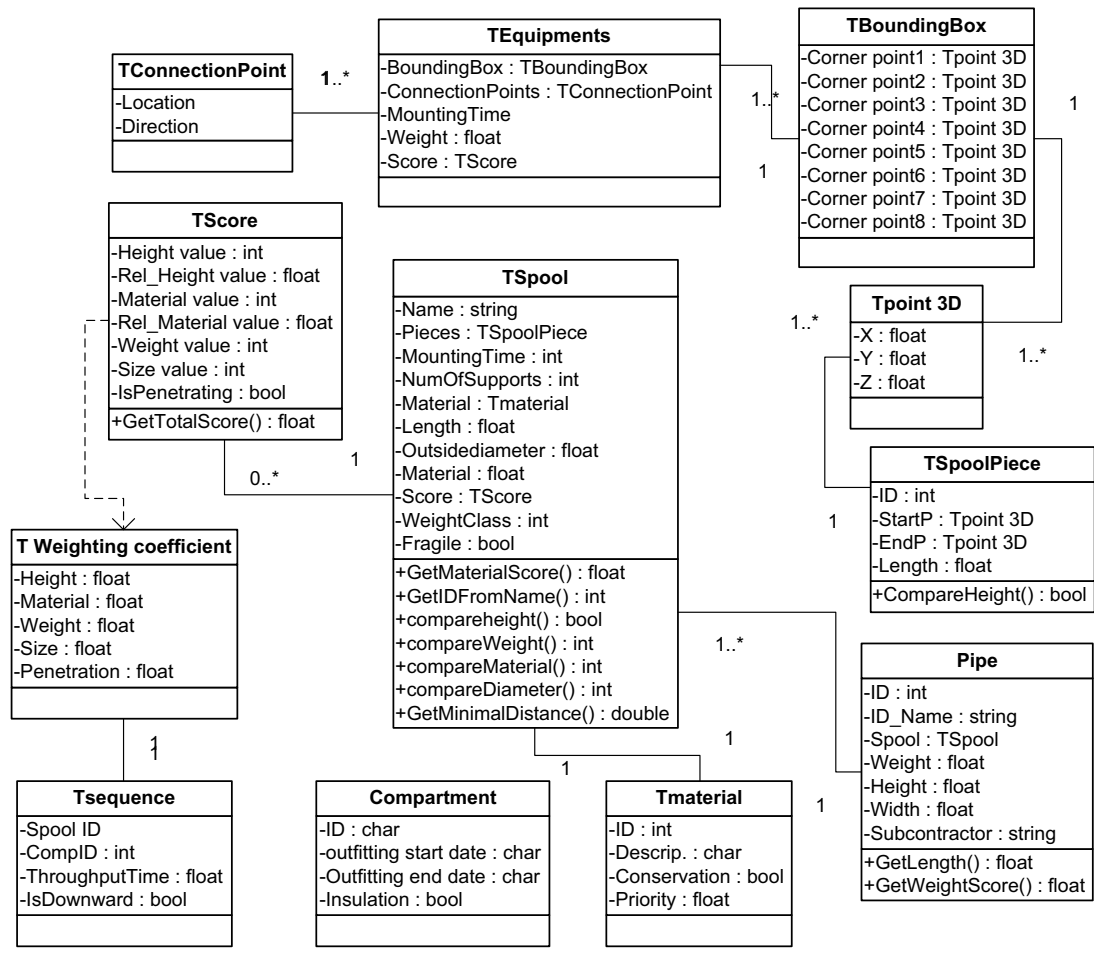

Figure C.1: An overview of data definition in UML class diagram 


\section{D}

\section{Flowchart of the Basket Done method}

The flowchart of the Basket Done Method describes this algorithm in detail. The tasks 'Find shortest Total Time (Basket 1)' and 'Put the spools with the shortest Total Time into Basket Done' are decomposed into smaller tasks shown in figure D.1. The complete flowchart is displayed in figure D.2 and D.3.
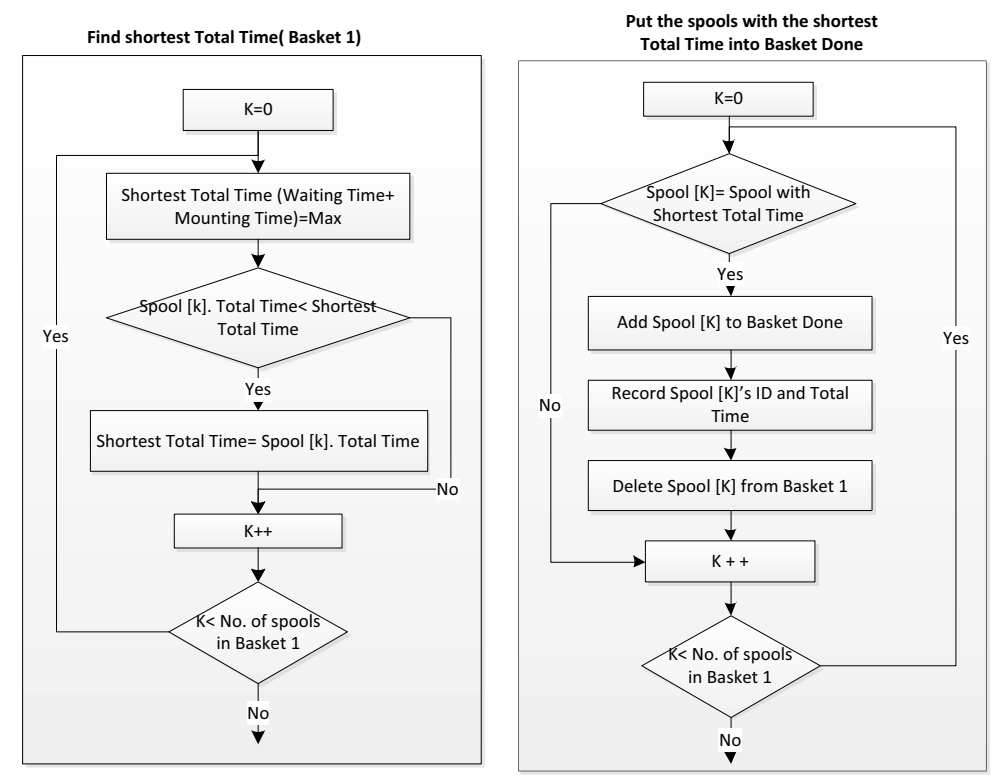

Figure D.1: Two tasks decomposed into smaller tasks 
172 Automatic generation of assembly sequence for the planning of outfitting processes in shipbuilding

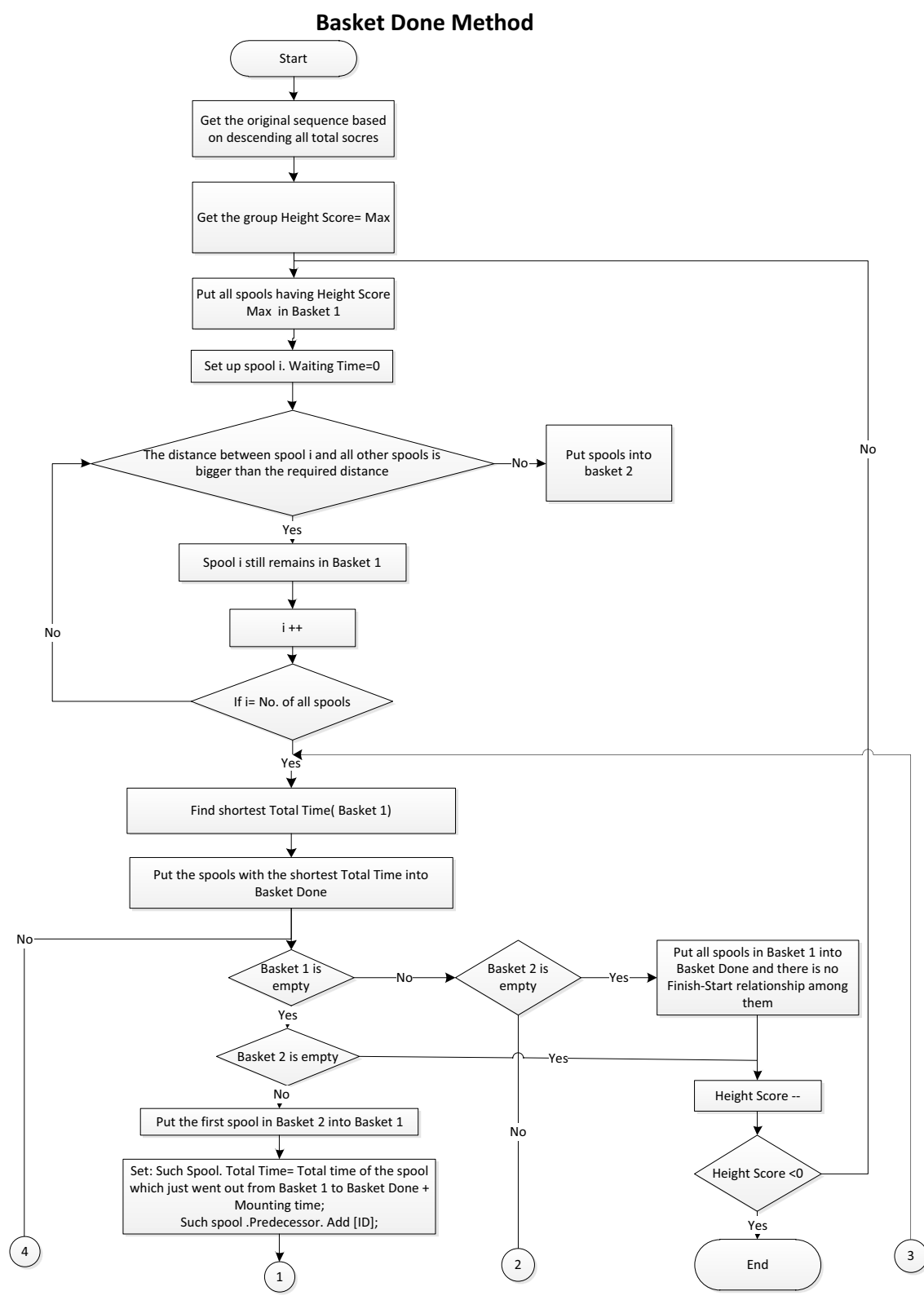

Figure D.2: The flowchart of the Basket Done Method (1) 


\section{Basket Done Method}

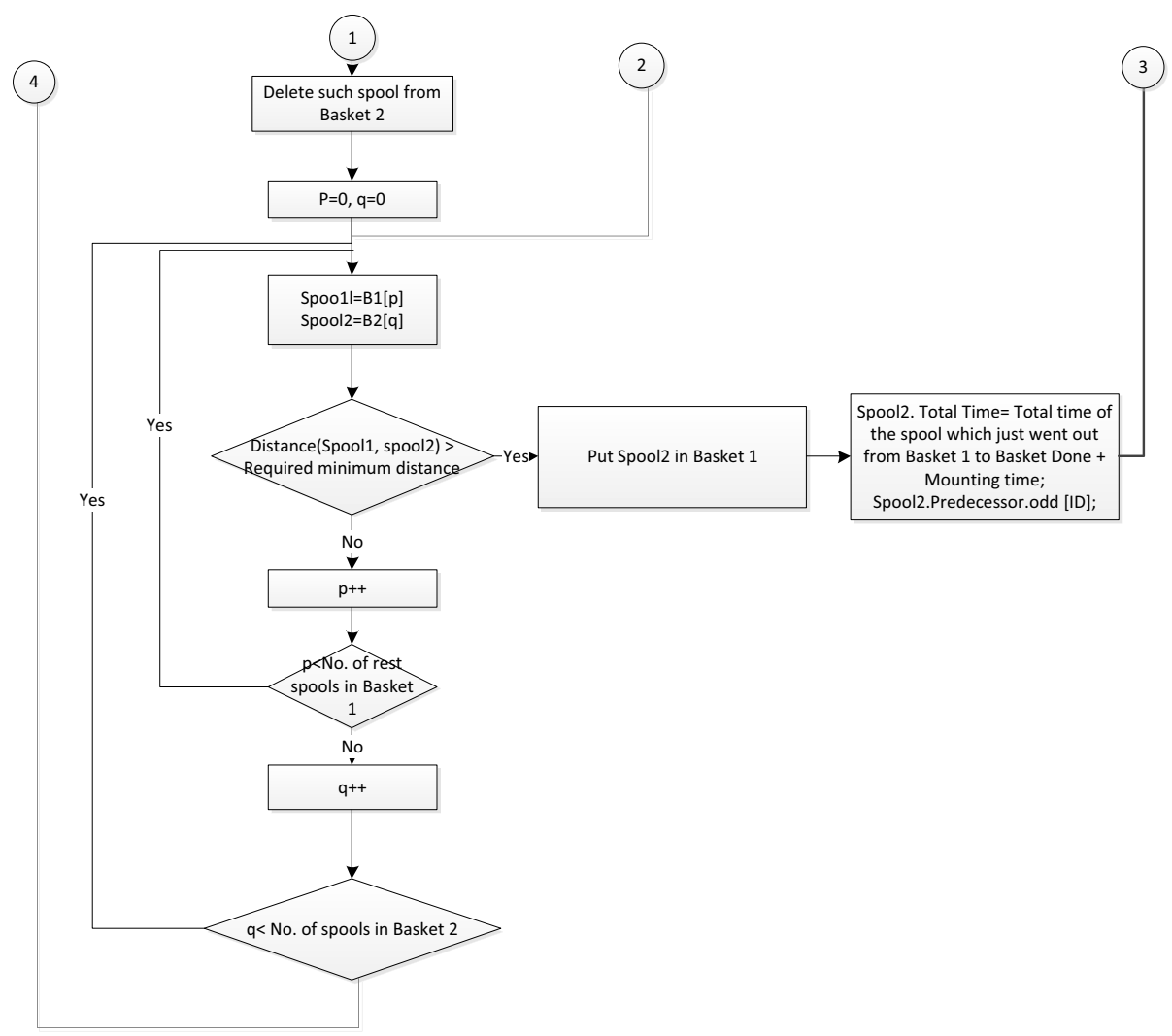

Figure D.3: The flowchart of the Basket Done Method (2) 

$\mathbf{E}$

\section{The background of the questionnaire people}

The background of the questionnaire people is introduced in table E.1.

Table E.1: The background of the questionnaire people

\begin{tabular}{lllc}
\hline Name & Age & Specialization & Years of working \\
\hline A.v.d.Velden & 39 & Pipe fitter & 16 \\
Y.C.Mosteiro & 44 & Pipe fitter & 21 \\
A. Batman & 33 & Production project manager & 3 \\
R. van Vugt & 52 & Production supervisor & 2 \\
J. Lemson & 64 & Chef Pre-outfitting coordinator & 20 \\
D. de Bruin & 38 & Electrical supervisor & 22 \\
\hline
\end{tabular}





\section{$\mathbf{F}$}

\section{The animation of the assembly sequence of test case $\mathrm{S1}$}

The animation of the assembly sequence of section S1, the work shop engine room section, is displayed in detail here. The following screen shot images show scenarios when different number of components have been assembled.

Different colors in the pictures represent types components and are explained here:

Gray- Steel structure

Red-Pipes

Blue- Cable Trays

Pink- Spiro-ducts

Green- Rectangular ducts

Yellow- Equipment

Transparent color- When the color of a component is transparent, it means that this component is in the process of being assembled. When its assembly is finished, the component is fully colored. 
178 Automatic generation of assembly sequence for the planning of outfitting processes in shipbuilding

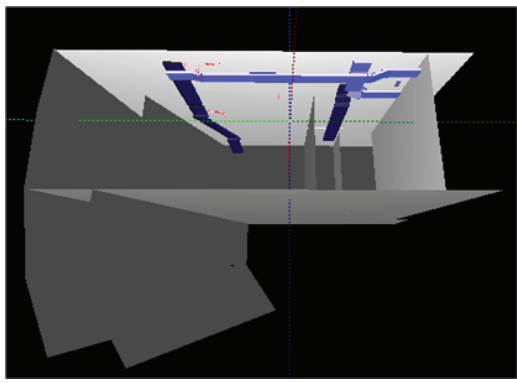

(a) 10 components

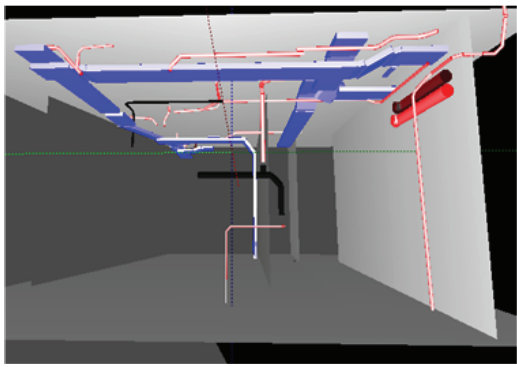

(c) 35 components

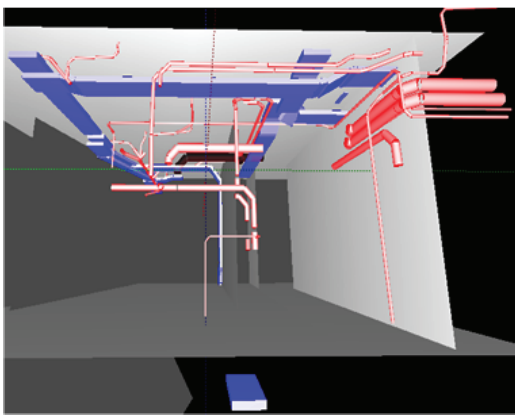

(e) 62 components

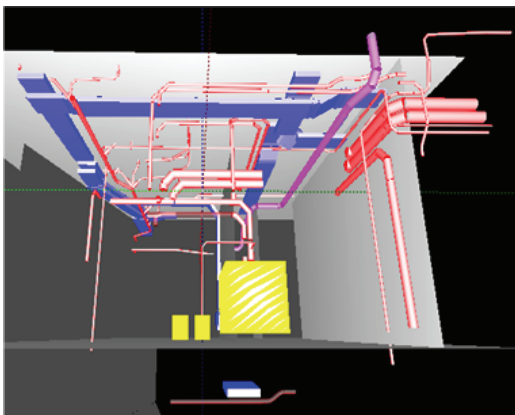

(g) 91 components

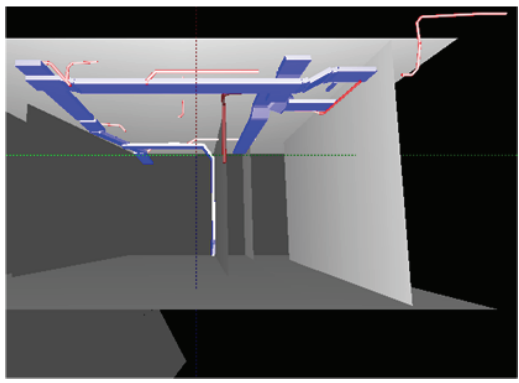

(b) 20 components

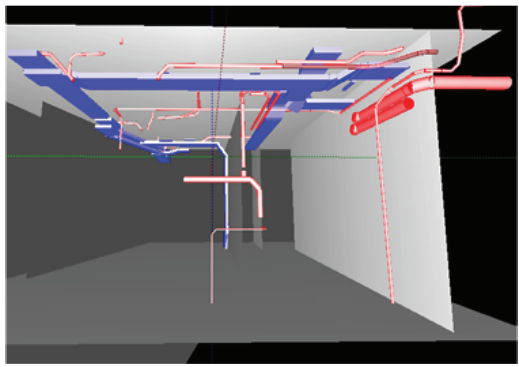

(d) 44 components

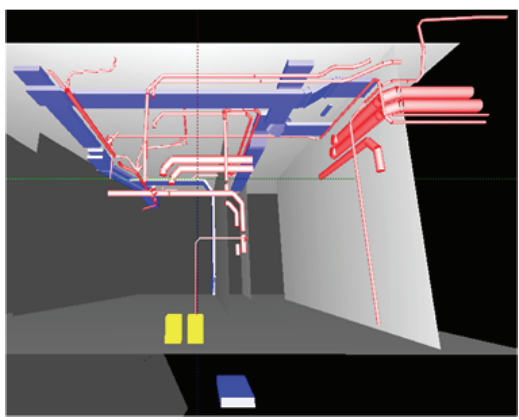

(f) 71 components

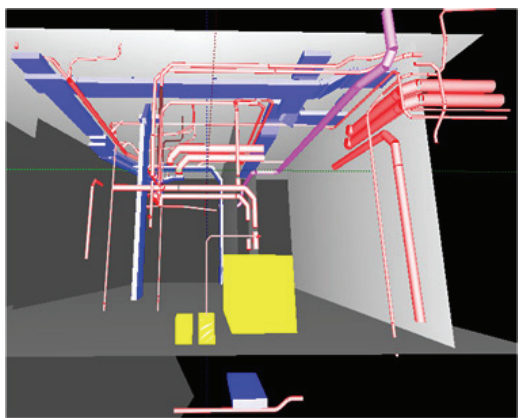

(h) 103 components

Figure F.1: Stages in the animation 


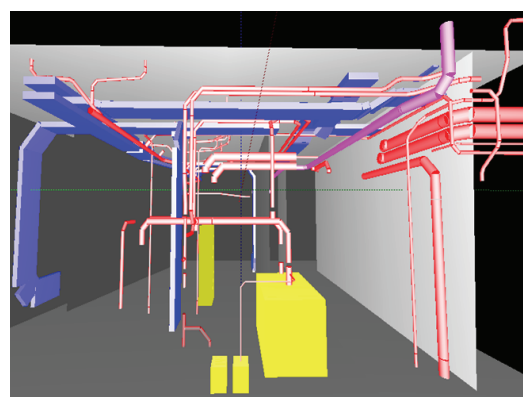

(a) 113 components

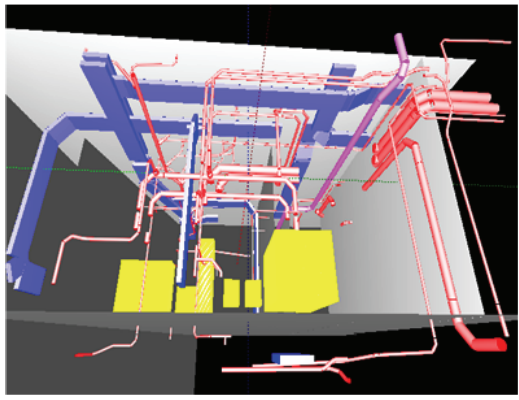

(c) 150 components

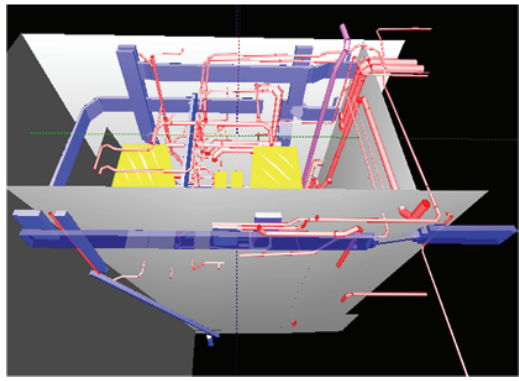

(e) 178 components

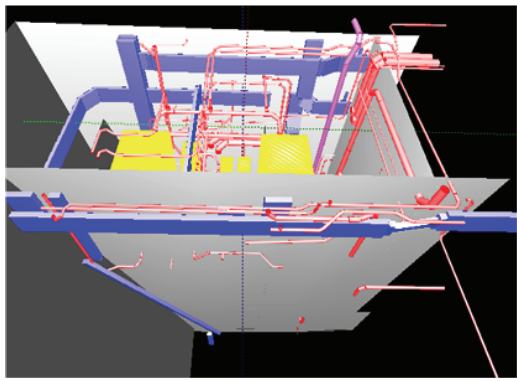

(g) 204 components

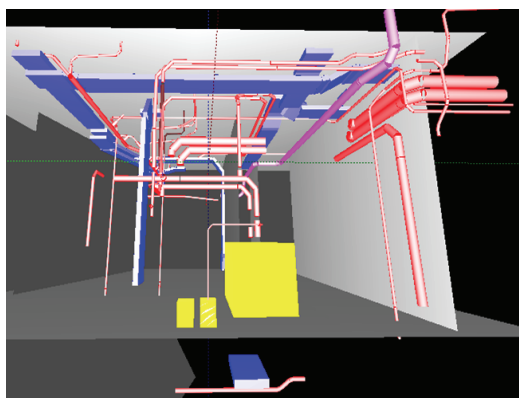

(b) 133 components

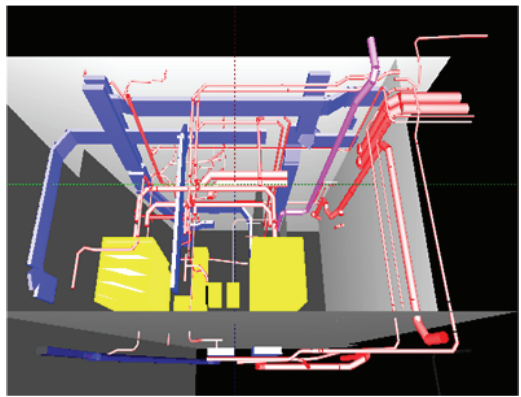

(d) 163 components

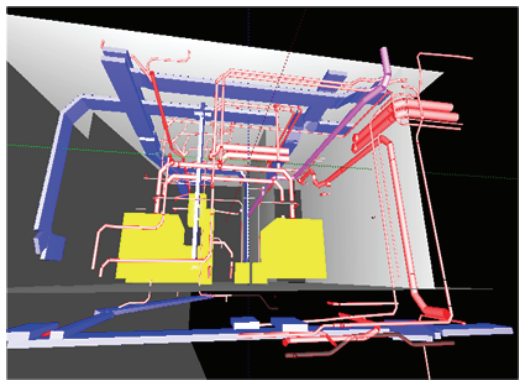

(f) 191 components

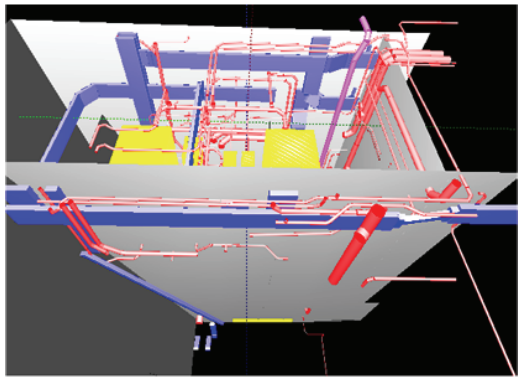

(h) 230 components

Figure F.2: Stages in the animation 
180 Automatic generation of assembly sequence for the planning of outfitting processes in shipbuilding

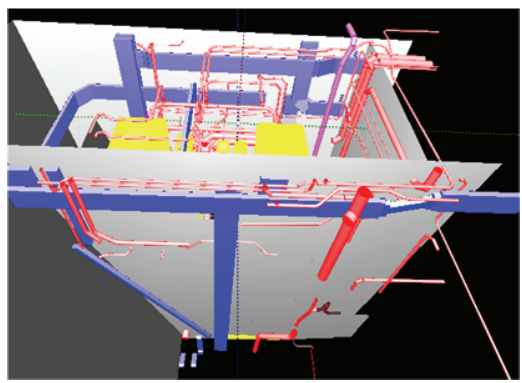

(a) 253 components

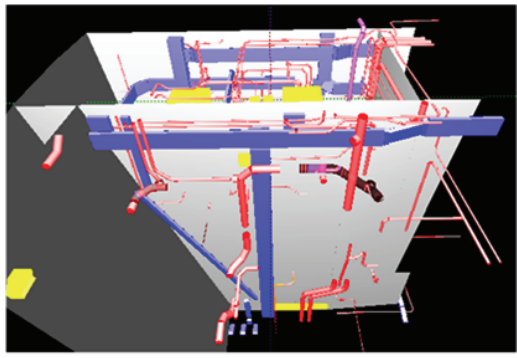

(c) 301 components

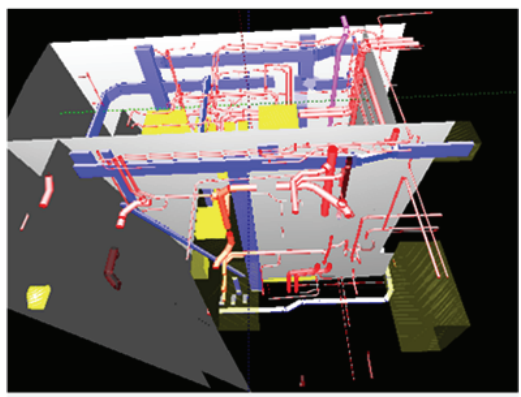

(e) 352 components

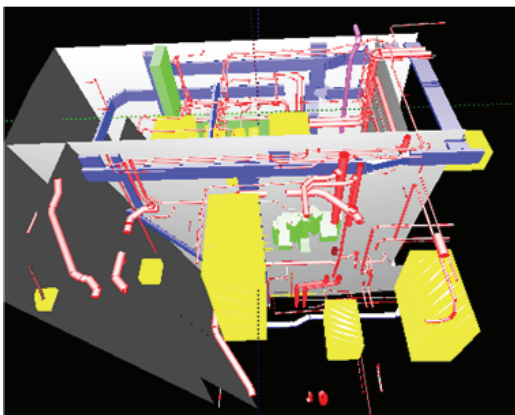

(g) 419 components

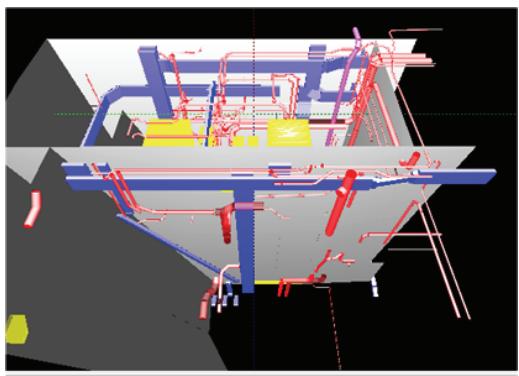

(b) 280 components

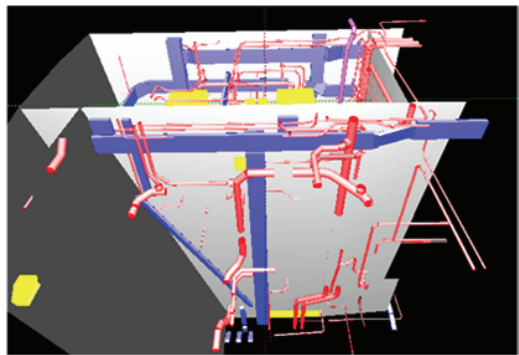

(d) 326 components

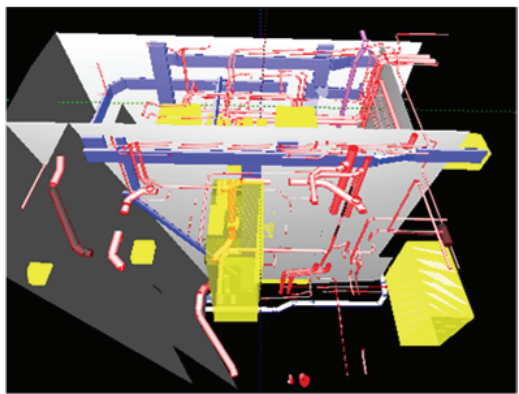

(f) 385 components

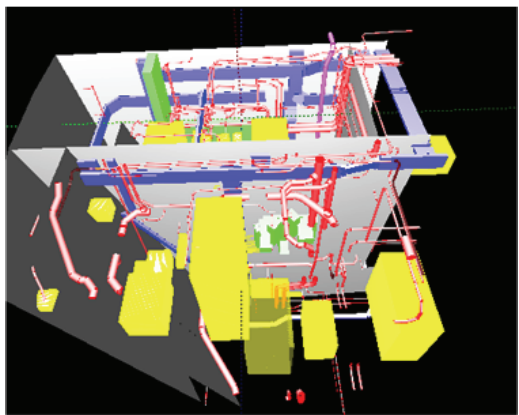

(h) 440 components

Figure F.3: Stages in the animation 


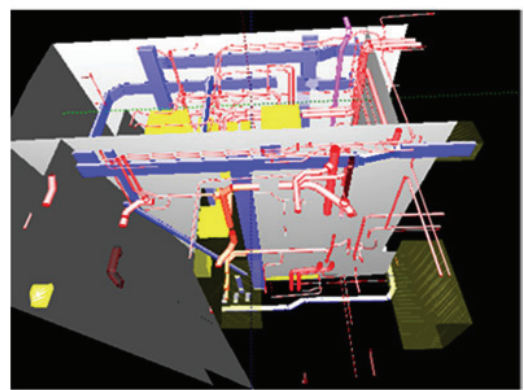

(a) 469 components

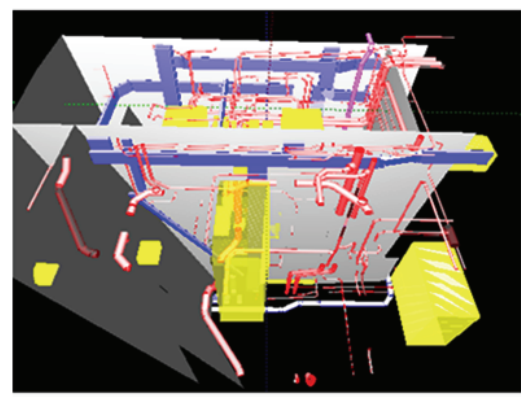

(b) 490 components

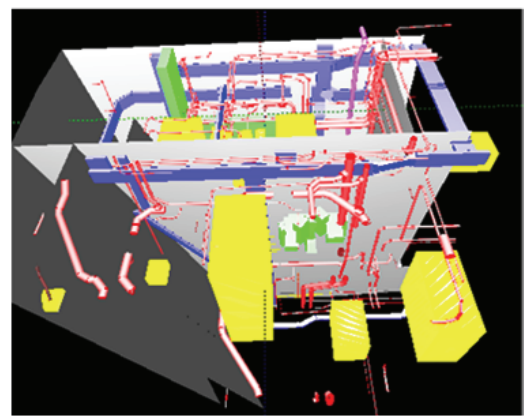

(c) 502 components

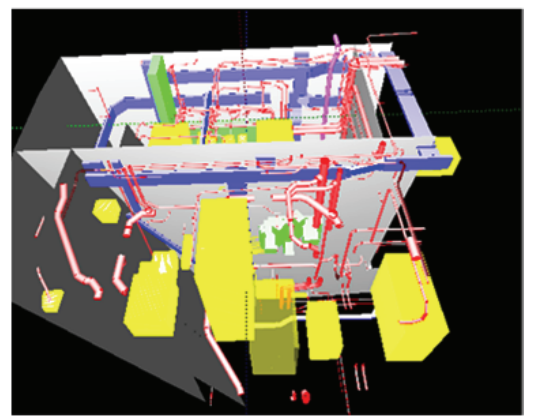

(d) 522 components

Figure F.4: Stages in the animation 



\section{Bibliography}

B. E. Altic, R. M. Burns, B. J. Fontaine, I. Scott, J. L. Silveira, and J. Softley. Implementation of an improved outfit process model. Journal of Ship Production, Vol.19, No. 1, February:1-7, 2003.

F. Andritsos and J. Perez-prat. The automation and integration of production processes in shipbuilding. European Commission, Joint Research Center, Institute for Systems, Informatics and Safety, 2000.

A. Asmara. Phd thesis-automatic pipe routing for shipbuilding. forth coming, 2012.

A. Asmara and U. Nienhuis. Automatic piping system in ship. 5th International conference on computer and IT Applications in the Maritime Industries (COMPIT), pages 269-279, 2006.

A. Asmara and U. Nienhuis. Automatic piping system implementation: a realy case. 6th International conference on computer and IT Applications in the Maritime Industries (COMPIT), pages 290-299, 2007.

A. Asmara and U. Nienhuis. Optimum routing of piping in real ships under real constraints. 7th International conference on computer and IT Applications in the Maritime Industries (COMPIT), pages 271-281, 2008.

ASME. Guide for verification and validation in computational solid mechanics. Technical report, the American Society of Mechanical Engineers, 2006.

K. A. Asok and K. Aoyama. Module division planning considering uncertainties. Journal of Ship Production, Vol.25, No. 3:153-160, 2009.

R. Baade, F. Kling, K. Lynaugh, F. Woronkowicz, and K. Seidler. Modular outfitting. The National Shipbuilding Research Program 1997 Ship Production Symposium, pages 1-18, 1997.

D. Ben-Arieh. A methodology for analysis of assembly operations' difficulty. International Journal of Production Research, 32,No.8:1879-1895, 1994. 
184 Automatic generation of assembly sequence for the planning of outfitting processes in shipbuilding

D. Ben-Arieh and B. Kramer. Computer-aided process planning for assembly: generation of assembly operation sequence. International Journal of Production Research, 32,No.3:643-656, 1994.

P. M. Berry. Satisfying conflicting objectives in factory scheduling. Artificial Intelligence Applications, Vol. 1:101-107, 1990.

N. Bhushan and K. Rai. Strategic Decision Making: Applying the Analytic Hierarchy Process (Decision Engineering). London: Springer-Verlag, 2004.

B. W. Boehm. Software engineering and economics. Prentice Hall, 1981.

C. A. Boer, A. Verbraeck, and H. P. M. Veeke. Distributed simulation of complex systems: application in container handling. Simulation interoperability workshop EURO SIW 2002, pages 1-9, 2002.

A. Bourjault. Contribution a une approache methodologique de l'assemblyage automatise: Elaboration automatique des sequences operatories. PhD thesis, 1984.

E.B. Brosens. Simulation of outfitting processes. Delft University of Technology, 2008.

H. J. Bullinger, P. J. Rally, and J. Schipfer. Some aspects of ergonomics in assembly planning. International Journal of Industrial Ergonomics, 20:389-397, 1997.

B. M. Caldwell. Ship building contracts. Marine Life, 2002.

J. Coenen. Controlling Engineering-to-Order Process in Shipbuilding. Delft University of Technology, 2008.

T. L. De Fazio and D. E. Whitney. Simplified generation of all mechanical assembly sequences. IEEE Journals of Robotics and Automation, Vol.RA-3, No.6:640-658, 1987.

R. L. Diesslin. A conceptual information model (data base design) for outfit planning. National Shipbuilding Research Project, 1982.

V. Dlugokecki, D. Fanguy, L. Hepinstall, and D. Tilstrom. Transforming the shipbuilding and ship repair project environment. SNAME Conference, pages 1-10, 2009.

Erico. Pipe hanger and support recommended specifications. ERICO International Cooperation, 2009.

H. Eriksson. UML 2 Toolkit. Wiley, 2004.

F. Frenken. Average dutchman just as tall as in 2001. Web magazine on 'Statistics Netherlands' website, 2008. 
T. Galnur. Business planning levels: Strategic, tactical, and operational. http://managingabusiness.com/businessplanning, 2005.

N. Granger. Demographic change and skills requirements in the shipbuilding and ship repair industry. Report of the HR Workshop, 2008.

R. J. Graves and L. F. McGinnis. The outfitting planning problem: Production planning in shipbuilding. Naval Research Logistics Quarterly, 20, No. 2:257-384, 1982.

ECORYS SCS Group. Study on competitiveness of the european shipbuilding industry. Directorate-General Enterprise and Industry, page 117, 2009.

P't. Hart and D. Schotte. Demographic change and skills requirements in the shipbuilding and ship repair industry. European Shipbuilding Social Dialogue Committee-HR Research Study, 2008.

G. Hoffmann. Distance between line segments. http://www.fho-emden.de/ hoffmann/xsegdist03072004.pdf, 2004.

L. S. Homem de Mello and A. C. Sanderson. And/or graph representation of assembly plans. IEEE Transactions on Robotics and Automation, Vol.6, No.2:188-199, 1990.

L. S. Homem de Mello and A. C. Sanderson. Representation of mechanical assembly sequences. IEEE Transactions on Robotics and Automation, Vol.7, No.2:211-227, 1991a.

L. S. Homem de Mello and A. C. Sanderson. A correct and complete algorithm for the generation of mechanical assembly sequences. IEEE Transactions on Robotics and Automation, Vol.7, No.2:228-240, $1991 \mathrm{~b}$.

J. J. Hopman. Innovation focused ship design-developments and perspectives from a european perspective. DSID2 Conference Proceedings, Delft, the Netherlands: 111-124, 2007.

Y. F. Huang and C. S. G. Lee. Precedence knowledge in feature mating operation assembly planning. IEEE International Conference on Robotics and Automation, pages 216-221, 1989.

P. Hughes and E. Ferret. Introduction to Health and Safety in Construction. Elsevier Ltd., 2008.

L. T. Hung. Virtual simulation in automotive industry-fe-based fatigue simulation of seam welds on car components and body. Simulation interoperability workshop EURO SIW 2002, pages 1-9, 2002. 
186 Automatic generation of assembly sequence for the planning of outfitting processes in shipbuilding

IKEI. Managing cyclical change in the european shipbuilding and ship repair industries. Comprehensive sectoral analysis of emerging competencies and economic activities in the European Union: building and repairing of ships and boats sector, 2009.

C. S. Jonson and L. D. Chirillom. Outfitting planning. U.S. Department of Commerce Maritime Administer in cooperation with Todd Pacific Shipyards Corporation, 1979.

J. Kaarsemaker and U. Nienhuis. Simulation of a maritime pre-fabrication process. proceedings of COMPIT, Oegstgeest:419-431, 2006.

M. Konig, U. Beibert, D. Steinhauer, and H. Bargstadt. Constraint-based simulation of outfitting processes in shipbuilding and civil engineering. 6th EUROSIM Congress on Modeling and Simulation, 2007.

T. Lamb. Ship Design and Construction. SNAME, 2004.

LeaderSHIP2015. Report of the high level advisory group. 2003.

B. Liu. Problem acquisition in scheduling domains. Expert Systems with Applications, Vol. 6, Issue 3:257-265, 1993.

P. Maffioli, J. C. Daidola, and J. Olivier. Competitive shipbuilding production practices. Transactions- Society of Naval Architects and Marine Engineers 109, pages 309-339, 2001.

N. L. Maziero, J. C. E. Ferreira, and F. S. Pacheco. A method for the automatic identification of contacts in assemblies of cyclindrical parts. Journal of the Brazilian Society of Mechanical Sciences and Engineering, Vol. XXVI, No. 3:297-307, 2004.

Sheri McConnell. 7 mental marketing principles for authors and solo professionals. 2006.

L. F. McGinnis and R. J. Graves. The outfitting planning problem. Proceedings of the REAPS Technical Symposium, Philadelphia, October 14-16:197-207, 1980.

T. Muller. Managing cyclical change in the european shipbuilding and ship repair industries. Stakeholder enquiry service, European Foundation for the Improvement of Living and Working Conditions, 2007.

S. Y. Nof, W .E. Wilhelm, and H. J. Warnecke. Industrial assembly. 1997.

E. S. Popko and C. Barlach. Digital manufacturing: The virtual shipyard. Setting the Course and Strategy for Digital Shipbuilding, pages 1-32, 2003. 
R. Rubesa. Procedure for estimating the effectiveness of ship modular outfitting. Report, pages 55-62, 2011.

T. Saaty. The analytica hierarchy process: Planning, priority setting, resource allocation. 1980.

A. Saltelli and M. Ratto. Global Sensitivity Analysis-the primer. John Wiley and Sons, 2008.

M. Santochi and G. Dini. Automated sequencing and subassembly detection in assembly planning. Annals of the CIRP, 41:1-4, 1992.

R.G. Sargent. Verification and validation of simulation models. Proceedings of the 2005 Winter Simulation Conference, pages 130-143, 2005.

J. F. Schank, H. Pung, G. T. Lee, M. V. Arena, and J. Birkler. Outsourcing and outfitting practices. Implications for the Ministry of Defense Shipbuilding Programs, RAND Corporation, 2004.

S. Sharma, B. B. Biswal, P. Dash, and B. B. Choudhury. Generation of optimized robotic assembly sequence using ant colony optimization. 4th IEEE Conference on Automation Science and Engineering, pages 894-899, 2008.

A. M. Sirajuddin. An automated project planner. Department of Civil Engineering, University of Nottingham: PhD Thesis, 1991.

L. P. A. Steenbekkers and C. E. M. van Beijsterveld. Design-relevant Characteristics of Ageing Users. Delft University of Technology, 1998.

D. Steinhauer. Sapp-simulation aided production planning at flensburger. Int. Conf. Computer Applications and Information Technology in the Maritime Industries (COMPIT), Hamburg:391-398, 2005.

D. Steinhauer. Simulation in shipbuilding-support for shipyard development, production planning and ship design at flensburger shipyard. 12th ASIM Dediated Conference on Simulation in Production and Logistics, pages 1-14, 2006.

D. Steinhauer. The simulation toolkit for shipbuilding (sts) 10 years of cooperative development and interbranch applications. Int. Conf. Computer Applications and Information Technology in the Maritime Industries (COMPIT), Berlin:453-465, 2011.

Q. Su. A hierarchical approach on assembly sequence planning and optimal sequences analyzing. Robotics and Computer-Integrated Manufacturing, pages 1$11,2008$. 
188 Automatic generation of assembly sequence for the planning of outfitting processes in shipbuilding

W. Tann and H.J. Shaw. The implementation method of data sharing based on ship production modeling. Journal of Taiwan Society of Naval Architects and Marine Engineers, 26, No.1:9-17, 2007.

K. Teknomo. Analytic hierarchy process (ahp) tutorial. http://people.revoledu.com/kardi/tutorial/ahp, 2006.

W. Thabet and Y. Beliveau. Modeling work space to schedule repetitive floors in multistory buildings. ASCE Journal of Construction Engineering and Management, 120(1):96-116, 1994.

Y. J. Tseng, J. F. Jhang, and F. Y. Huang. Multi-plant assembly planning models for a collaborative manufacturing environment. International Journal of Production Research, 45, No.15:3333-3349, 2007.

H. van Alphen, H. A. Guyt, U. Nienhuis, and J. C. van der Wagt. Virtual manufacturing in shipbuilding processes. Conf. European Shipbuilding, Repair and Conversion- The Future, London, 2004.

H. P. Wang and J. K. Li. Computer-aided process planning. pages 291-296, 1997.

T. C. Woo. Automatic disassembly and total ordering in three dimensions. Technical report, pages 1-38, 1987. 


\section{List of Abbreviations}

$\begin{array}{ll}\text { AHP } & \text { Analytical Hierarchy Process } \\ \text { BOM } & \text { Bill Of Material } \\ \text { CAD } & \text { Computer-Aided Design } \\ \text { CAPP } & \text { Computer Aided Process Planning } \\ \text { CI } & \text { Consistency Index } \\ \text { DB } & \text { Data Base } \\ \text { DFA } & \text { Design for assembly } \\ \text { DSS } & \text { Decision Support System } \\ \text { DXF } & \text { Data eXchange Format } \\ \text { ERW } & \text { Electric Resistance Welded } \\ \text { HVAC } & \text { Heating Ventilation Air Conditioning } \\ \text { LT } & \text { Low Temperature } \\ \text { PVC } & \text { PolyVinyl Chloride } \\ \text { SA } & \text { Sensitivity Analysis } \\ \text { UML } & \text { Unified Modeling Language } \\ \text { XML } & \text { Extensible Markup Language }\end{array}$





\section{List of Figures}

1.1 Awkward work position to install HVAC ducts . . . . . . . . . 4

1.2 Collision between the support of cable trays and steel pipe . . . . . 4

1.3 EU-14 Shipbuilding technical workforce-age distribution (outlook over 10-15 years) (reproduced from Hart (Hart and Schotte, 2008)) 5

1.4 Search pipe spools in a pipe pallet . . . . . . . . . . 6

2.1 Main stages in shipbuilding process _... . . . . . . . . 12

2.2 Use case diagram for outfitting entities . . . . . . . . . . . . . . . 18

2.3 The worker burns the pipe holes . . . . . . . . . . . . . 19

3.1 Phases of production planning $\ldots \ldots \ldots \ldots$

3.2 How to make the production planning $\ldots \ldots \ldots \ldots$

3.3 Planning system for outfitting processes . . . . . . . . . 34

4.1 A simple product in exploded view (reproduced from Homem (Homem de Mello and Sanderson, 1990)) . . . . . . . . . . . . . . . 43

4.2 Example of an assembly sequence (reproduced from Homem (Homem de Mello and Sanderson, 1990)) . . . . . . . . . . . . . . . . . 44

4.3 Physical contacts among components of a caster . . . . . . . . . . 46

4.4 No physical contacts between two pipe spools in a section . . . . 47

4.5 An example of assembly consisting of 7 components . . . . . . 48

4.6 Liaison diagram of 7 components . . . . . . . . . . . . . . 48

4.7 The assembly sequence of 7 components based on the liaison diagram 49

4.8 Example of an assembly . . . . . . . . . . . . . . 50

4.9 Various tree structures of the assembly with four components . . . 52

4.10 The AND/OR graph of the assembly with 7 components . . . . . 53

5.1 The structure of assembly sequence generation system _ . . . . . 59

5.2 A supporting tool for outfitting planning and outfitting assembly . . 61

6.1 Pipes are mounted in a certain sequence . . . . . . . . . . . . 64

6.2 Attributes of outfitting components . . . . . . . . . . . . . 64 
192 Automatic generation of assembly sequence for the planning of outfitting processes in shipbuilding

6.38 components in a steel section . . . . . . . . . . . . . . 65

6.4 The front view of the steel section . . . . . . . . . . . . 66

6.5 A piece of pipe and equipment with their coordinates . . . . . . 67

6.6 Cartesian coordinate system of a ship . . . . . . . . . . . . 67

6.7 Width of rectangular ducts and cable trays . . . . . . . . . . 70

6.8 Two extreme scenarios of simultaneously assembling two spools . . 71

6.9 Examples of work position . . . . . . . . . . . . . . . . 72

6.10 Envelope of horizontal reaching (reproduced from Steenbekkers (Steenbekkers and van Beijsterveld, 1998)) . . . . . . . . . . . . 73

6.11 The minimum distance set as $2.5 \mathrm{~m} \ldots \ldots \ldots \ldots$. . . . . . . . 74

6.12 A test case with 8 components . . . . . . . . . . . 76

6.13 The procedure of interference detection along $+\mathrm{Z}$ direction $\ldots . .76$

6.14 The procedure of interference detection along $-Z$ direction $\ldots . . .77$

6.15 An example of two spools having the same $\mathrm{Z}$ coordinates but different $X$ coordinates . . . . . . . . . . . . . . . . . . . 77

6.16 An example of the endless iteration problem . . . . . . . . . 78

6.17 Scale to measure the level of importance . . . . . . . . . . . . 81

6.18 Relative importance of physical attributes . . . . . . . . . . 82

7.1 An example of $x m l$ file produced by Tribon . . . . . . . . . . 87

7.2 A piece of HVAC duct corresponding to the XML file in figure 7.1 . 88

7.3 Raw data from the detailed engineering model . . . . . . . . . . 88

7.4 Functions of data preparation model . . . . . . . . . . . . . 89

7.5 An example of cutting HVAC ducts . . . . . . . . . . . . 90

7.6 The geometrical information of the duct in data preparation model . 90

7.7 An example of splitting a piece of HVAC duct into 5 spools _ . . . 91

7.8 Ducts with couplings . . . . . . . . . . . . . . 93

7.9 The position of equipment boundary box . . . . . . . . . . 95

8.16 steps on the operating screen of the model . . . . . . . . . . . 100

8.2 A test case with 14 components . . . . . . . . . . . . . . . . . 101

8.3 Distance calculation between any two components in the 14-component test case . . . . . . . . . . . . . . . . . . . 103

8.4 Illustration of Basket Done Method . . . . . . . . . . . . . . . 104

8.5 The assembly sequence of seven components after the Basket Done

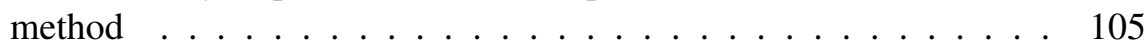

8.6 The final assembly sequence of 14-component in Gantt Chart . . . . 107

8.7 The final assembly sequence of the 14-component case in 3D animation 108

8.8 The human resource distribution according to the assembly sequence of 14 -component . . . . . . . . . . . . . . . . . . . 110

8.9 The minimum duration of the 14-component case influenced by the number of workers 
8.10 The human resource distribution with 2,4 and 6 workers . . . . . . 112

8.11 Sequence determination of components according to their categories 114

9.1 Two pipe spools in a section _. . . . . . . . . . . . . . . . 118

9.2 Answers from the questionnaires: estimation of mounting time of spool 1 and $2 \ldots \ldots$. . . . . . . . . . . . . . . . . . 119

9.3 Two HVAC ducts in a section . . . . . . . . . . . . . . . 121

9.4 Answers from the questionnaires: estimation of mounting time of duct 1 and $2 \ldots \ldots$. . . . . . . . . . . . . . 122

9.5 Two cable trays in a section . . . . . . . . . . . . . . . 123

9.6 Answers from the questionnaires: estimation of mounting time of cable tray 1 and $2 \ldots \ldots$. . . . . . . . . . . . 124

9.7 A test case with 10 components . . . . . . . . . . . . . . 125

9.8 Downward and upward version: Assembly sequence of 10-component test case . . . . . . . . . . . . . . . . . . 126

$9.93 \mathrm{D}$ visualization of the complete section $\mathrm{S} 1 \ldots \ldots$. . . . . . . . 127

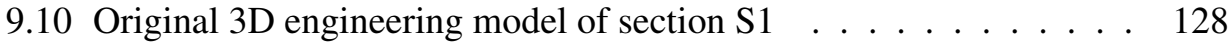

9.11 The complete assembly sequence of section $\mathrm{S} 1$. . . . . . . . . . . 129

9.12 A pipe spool is near the edge of the steel section . . . . . . . . . . . 131

9.13 A cable tray penetration piece and its connected cable trays . . . . . 132

9.14 A central heating boiler and its connected spool . . . . . . . . . . 133

9.15 Two pipelines in the model . . . . . . . . . . . . . . . . . . 134

9.16 The assembly order of pipe spools on the two pipelines . . . . . . . 134

9.17 Small iron work of the test case S1 (not included in the assembly sequence generation model) . . . . . . . . . . . . . 135

9.18 Insulation area of the test case $\mathrm{S} 1 \ldots$. . . . . . . . . . . . 136

9.19 The possible way to generate a more realistic equipment installation sequence . . . . . . . . . . . . . . . . . 142

9.20 Couple compartments with sections . . . . . . . . . . . 143

10.1 The comparison of importance levels . . . . . . . . . . . . . . . . . 146

10.2 A test case with 14 components . . . . . . . . . . . . . . 150

10.3 Position influence . . . . . . . . . . . . . . . . . . . 151

10.4 Material influence . . . . . . . . . . . . . . . 151

10.5 Weight influence . . . . . . . . . . . . . . . 152

10.6 Size influence . . . . . . . . . . . . . . . . . 153

10.7 Penetration influence . . . . . . . . . . . . . . . 153

10.8 A test case with 10 components . . . . . . . . . . . . . 155

10.9 Distance calculation between any two components in the 10-component test case . . . . . . . . . . . . . . . . . . . 155 
194 Automatic generation of assembly sequence for the planning of outfitting processes in shipbuilding

A.1 Preference level between apple and banana (reproduced from Hart (Teknomo, 2006)) . . . . . . . . . . . . . . . . . . . . 164

A.2 Mark your preference (reproduced from Hart (Teknomo, 2006)) . . 164

C.1 An overview of data definition in UML class diagram . . . . . . . 170

D.1 Two tasks decomposed into smaller tasks _ . . . . . . . . . . 171

D.2 The flowchart of the Basket Done Method (1) . . . . . . . . . . 172

D.3 The flowchart of the Basket Done Method (2) . . . . . . . . . . . . 173

F.1 Stages in the animation . . . . . . . . . . . . . 178

F.2 Stages in the animation . . . . . . . . . . . . . . . . . . 179

F.3 Stages in the animation . . . . . . . . . . . . . . . 180

F.4 Stages in the animation . . . . . . . . . . . . . . . 181 


\section{List of Tables}

6.1 Assembly ranking of 8 components $\ldots \ldots \ldots \ldots 2$

8.1 The mounting order in a sequential manner of 14 components obtained from step $1-4 \ldots \ldots \ldots$. . . . . . . . . . . . 102

8.2 The distance comparison of seven components . . . . . . . . . . 103

8.3 The final assembly sequence of 14 -component in text . . . . . . 106

9.1 Geometrical attributes of two pipe spools . . . . . . . . . . 118

9.2 Answers from the questionnaires: estimation of the number of supports of pipe spool 1 and $2 \ldots \ldots \ldots \ldots$

9.3 Geometrical attributes of two ducts . . . . . . . . . . . . . 120

9.4 Geometrical attributes of two cable trays . . . . . . . . . . . 121

9.5 Answers from the questionnaires: estimation of the number of supports of cable tray 1 and $2 \ldots \ldots \ldots \ldots$. . . . . . . . . . . . . . . . . 121

9.6 Choose the better assembly sequence . . . . . . . . . . . . . . . 127

9.7 The comparison of duration . . . . . . . . . . . . . . . . 128

9.8 The duration of four test cases under different scenarios . . . . . . . 137

9.9 The difference in input sources for determining the mounting order and mounting time of equipment and other outfitting components . . 140

10.1 Position attribute: Weighting Coefficients under different importance level . . . . . . . . . . . . . . . . . . . . . 148

10.2 Position attribute: assembly sequences under different importance level149

10.3 Conclusion of the sensitivity analysis . . . . . . . . . . . . . 154

10.4 Duration time (minutes) influenced by the minimum work distance . 156

10.5 Maximum number of components mounted in parallel influenced by the minimum work distance . . . . . . . . . . . . . . . 156

10.6 Duration time comparison between two versions of assembly sequences 157

A.1 The pairwise comparison derived from the interviewers . . . . . 165

B.1 Estimate mounting time of pipe spools . . . . . . . . . . . 167 
196 Automatic generation of assembly sequence for the planning of outfitting processes in shipbuilding

B.2 Estimate mounting time of cable trays $\ldots \ldots \ldots \ldots$

E.1 The background of the questionnaire people . . . . . . . . 175 


\section{Summary}

The most important characteristics of the outfitting processes in shipbuilding are:

- The processes involve many interferences between yard and different subcontractors. In recent years, the use of outsourcing and subcontracting has become a widespread strategy of western shipyards. There exists not only the vertical relationship between a yard and a subcontractor but also the horizontal relationships between the subcontractors themselves.

- They require timely and detailed engineering information. Outfitting performance depends largely on the quality, quantity, and timeliness of technical information supplied by engineering.

- Much ambiguity and tacit knowledge exists especially in the outfitting processes. Planners quite often use rules of thumb abundantly to make important decisions and workers, who carry out daily outfitting assembly work, very largely rely on many years of experience.

All these make the planning of outfitting processes, which have not been sufficiently considered in practice, a great challenge to both shipyards and subcontractors.

The study presented in this thesis aims to develop an automatic sequence generation method which is able to give the yard and subcontractors a realistic and reliable outfitting plan that identifies the relationships among outfitting activities, estimates a realistic mounting time, and displays it by means of animation.

First an integral planning system for the outfitting processes is proposed. It has been divided into three interrelated steps: activity generation, sequence determination and schedule determination, trying to answer questions "What has to be accomplished?", "In what order will it be accomplished?", and "When will it be accomplished?" respectively. The first two steps comprise process planning and the last is scheduling. The focus of the research is to determine the assembly sequence of the outfitting components, answering the first two questions.

Different assembly sequence determination methods, attempted in the mechanical industry, are described and the possibility of their application in shipbuilding outfitting processes is discussed. These methods include liaison diagrams, assembly 
198 Automatic generation of assembly sequence for the planning of outfitting processes in shipbuilding

sequence diagrams, and/or graphs and the binary matrix method. The interference matrix, part of the binary matrix method, is provisionally selected to detect the relationships between outfitting components because of its practical applicability to the shipbuilding situation.

Next, the kind of geometry attributes of outfitting components that are important in the determination of their mounting orders is analyzed. After field observations and thorough consultation with workers, the decisive attributes of components-position, material, weight, size, penetration and minimum work distance- have been chosen. The way to describe these attributes mathematically with a purpose of making them programmable is introduced. Weighting coefficients are used to quantify the relative impact of the components' geometry attributes on the mounting orders. They are calculated using the Analytical Hierarchy Process (AHP) method, applied to questionnaire results.

Subsequently, data collection and preparation are reported. For our own laboratory purpose, the detailed engineering CAD model, generated by TRIBON, was chosen to be the original data source. A data preparation model is presented. It is necessary to reorganize the data in a proper format so that it can be read by the sequence generation model. Apart from this, it is also able to extract the necessary information.

The assembly sequence generation model is developed starting from the requirement that the sequence should follow automatically from the CAD model of a particular section or compartment in the ship. All physical attributes of a component and their relative importance in deciding its mounting order were modeled, which resulted in the derivation of finish-start relationships between components. Two types of outputs of the model lead themselves to validation:

- the numerical results for throughput time and individual mounting times;

- the detailed assembly sequence visualized by Gantt charts or by 3D animation.

The validation study is described to investigate the correctness of the estimation of the mounting time of assembling each type of components, followed by application of the method for four test cases. The validation of the total mounting time for these four representative sections/compartments indicates, but does not prove, that the estimates for the mounting time are realistic. Also the validation work supports the conclusion that the generated sequence is realistic but not flawless. Discussion of the model's result shows that the methodology does not yet consider all technical and organizational aspects of outfitting processes at the same time. In fact, given all the complexities, it is gratifying that the method yields already useful assembly sequences that provide a basis for a better planning method. Nevertheless, before the developed method can be implemented on the worksite, additional effort will be necessary both in gathering validation data and improving the model (including the integration of steel structure). 
In conclusion, an automatic assembly sequence generation model for the planning of outfitting processes is developed in the thesis. It already allows generation of interference-free and integral assembly sequences including their throughput time. The behavior of the model will further improve and become even more realistic by implementation of all three dimensions of interference-detection, an improved equipment-mounting algorithm and the integration of steel structure information. 



\section{Samenvatting}

Het outfittingsproces in de scheepsbouw wordt gekarakteriseerd door de volgende belangrijke kenmerken:

- Het proces wordt benvloed door de vele onderlinge relaties tussen de werf en toeleveranciers. De laatste tijd is het gebruikelijk dat westerse werven het outfittingsproces uit besteden naar toeleveranciers. Hierdoor bestaan er naast de verticale relatie tussen werf en toeleverancier ook horizontale relaties tussen de toeleveranciers onderling.

- Het outfittingsproces heeft op het juiste moment gedetailleerde engineering informatie van de tekenkamer nodig. De kwaliteit van het outfittingsproces wordt in grote mate bepaald door de kwaliteit, kwantiteit en tijdigheid waarmee de technische informatie wordt aangeleverd.

- Er wordt veel impliciete kennis bij het outfittingsproces gebruikt. Werkvoorbereiders gebruiken vaak vuistregels voor belangrijke beslissingen en de outfitters maken gebruik van hun, vaak jarenlange, werkervaring met het outfittingsproces.

Dit alles bij elkaar maakt het plannen van het outfittingsproces een grote uitdaging, voor zowel de werven als voor de toeleveranciers. In de praktijk komt het er vaak op neer dat het proces niet genoeg uitgedetailleerd wordt.

Het doel van de beschreven studie is het ontwikkelen van een methode voor het automatisch genereren van een installatievolgorde voor de werf en toeleveranciers. De methode dient betrouwbare en realistische planningen $\mathrm{n}$ doorlooptijden voor het outfittingsproces op te leveren, waarin de onderlinge relaties tussen componenten worden erkend. Daarnaast dient de methode het proces, zoals gegenereerd, weer te geven middels een computeranimatie.

Als eerste wordt een alles omvattend planningsconcept voor het outfittingsproces voorgesteld. Dit is onderverdeeld in drie onderling samenhangende stappen: het genereren van de activiteiten, het bepalen van de installatievolgorde en het bepalen van de planning. Hierbij dienen de volgende vragen respectievelijk beantwoord te worden: "wat dient er bereikt te worden?", "in welke volgorde dient dit gedaan te 
202 Automatic generation of assembly sequence for the planning of outfitting processes in shipbuilding

worden?" en "wanneer zal het werk gereed zijn?". De eerste twee stappen hebben betrekking op het outfittingsproces en de volgordebepaling daarin, de laatste stap betreft de daadwerkelijke planning. De nadruk van dit onderzoek ligt bij het bepalen van de installatievolgorde van het outfittingsproces en dus bij het beantwoorden van de eerste twee vragen.

Vervolgens wordt er een beschrijving gegeven van de verschillende methoden voor het bepalen van installatievolgordes, opgezet voor andere takken van industrie. Aansluitend wordt de toepasbaarheid daarvan in de scheepsbouw besproken. Deze methoden bevatten verbandoverzichten, diagrammen voor installatievolgorde en de binaire matrix methode. De interferentie matrix, onderdeel van de binaire matrix methode, wordt voorlopig geselecteerd voor de bepaling van de onderlinge relaties tussen de verschillende outfitting componenten, vanwege de praktische toepasbaarheid ervan in het scheepsbouwproces.

Daarna worden de geometrische kenmerken, die van belang zijn voor het bepalen van de installatievolgorde, geanalyseerd. Na observaties op de werkvloer en uitvoerige raadpleging met de outfitters zijn een aantal bepalende kenmerken gekozen, namelijk positie, materiaal, gewicht, grootte, doorvoer en minimaal vereiste afstand voor de werkers. De wijze waarop deze kenmerken wiskundig te beschrijven zijn wordt gentroduceerd. Weegfactoren worden gebruikt om de relatieve impact van de geometrische kenmerken op de installatievolgorde te bepalen. De weegfactoren zijn berekend door gebruik te maken van de Analytical Hierarchy Process (AHP) methode.

Hierna wordt de dataverzameling en datakoppeling beschreven. Voor dit onderzoek is gekozen voor een gedetailleerd CAD model (TRIBON) als data bron. De data dient in geschikt formaat omgezet te worden, zodanig dat het door het model ingelezen kan worden om vervolgens installatievolgordes te genereren.

Dit model is ontwikkeld vanuit de eis, dat de installatievolgorde van de componenten voor een bepaalde sectie of compartiment automatisch uit het CAD model dient te volgen. Alle fysieke kenmerken van een component, en hun weegfactoren met betrekking tot installatievolgorde, zijn gemodelleerd. Dit heeft geresulteerd tot bepaalde start-finish relaties tussen componenten. Het model heeft geleid tot twee soorten resultaten, oftewel output:

- de numerieke waardes voor de doorlooptijd en de individuele installatietijd van een component;

- visualisatie van de gedetailleerde installatievolgorde d.m.v. Gantt grafieken en 3D animaties.

Een validatie onderzoek is uitgevoerd om de correctheid van de schatting van de installatietijden voor alle typen componenten te analyseren De validatie van de installatietijden voor vier representatieve secties duidt aan, maar geeft geen sluitend 
bewijs, dat de schattingen voor de installatietijden correct zijn. Daarnaast ondersteunt de validatie de conclusie dat de gemaakte installatievolgorde realistisch is, maar niet per definitie zonder fouten is. Bespreking van de resultaten laat zien dat de methodologie nog niet alle technische en organisatorische aspecten van het outfittingsproces meeneemt. Gegeven alle complexe aspecten kan men zelfs tevreden zijn dat de methode nu al een bruikbare installatievolgorde kan genereren, welke de basis van een betere planning kan vormen. Desalniettemin, zullen verdere stappen genomen moeten worden voordat deze methodiek gemplementeerd kan worden op de werkplek; zoals het verkrijgen van meer validatie data en het verbeteren van het model zelf (door bijvoorbeeld de stalen secties toe te voegen).

Concluderend kan gezegd worden dat het onderzoek, beschreven in dit proefschrift, heeft geleid tot een model wat automatisch een installatievolgorde voor het outfittingsproces kan genereren. Het model levert installatievolgorden op waarin de componenten geen fysieke belemmeringen ondervinden en het model geeft tevens de doorlooptijden. Het model kan verder verbeterd en meer realistisch worden, door het verbeteren van de installatievolgorde van de uitrusting en door de toevoeging van de informatie van de stalen secties, alsmede door het toevoegen van alle drie de dimensies die van belang zijn bij het herkennen van fysieke belemmeringen van het installeren van componenten. 



\section{Acknowledgments}

This thesis had been made possible by Delft University of Technology (TUD), China Scholarship Council and IHC Merwede. I am grateful to these organizations for allowing me to conduct this research.

I am very grateful to those in TUD. First and foremost, I would like to express my gratitude to Ubald Nienhuis for his excellent guidance, his patience and encouragement during my hardest periods of both my $\mathrm{PhD}$ years and my life. I also would like to make a special reference to Erik Ulijn who provided me with valuable support of the programming work and also the invitation for pancakes almost every Tuesday evening. Jeroen Pruyn, the daily supervisor, provided advice during numerous discussions. Elena Moredo, my office mate, was ready to give me encouragement and warm hugs at any time, especially in the thesis-writing period. Ria Nieuwland Jobse, our secretary, cared for my life here as a foreigner. Peter de Vos shared his marine engineering knowledge. Robert Hekkenberg encouraged me and Guus van der Bles inspired me and motivated me throughout the whole study period. Tomas DeNucci shared the experience of working as a PhD candidate at TUD. I owe my great gratitude to these dear colleagues.

I also would like to thank people at IHC Offshore \& Marine B.V. who provided invaluable help and supports. Especially, I thank Johan van Vuuren, the production manager and Jeroen Kaarsemaker, the manager process management. Without their support, the field study and all kinds of interviews could not have been carried out. I am also indebted to Frank Dekker, the system Manager CAD/CAM. He made major parts of the input information for the research available. Thanks to Andi Asmara, who not only shared his pipe routing knowledge but also provided equipment input information for the research. Many thanks to Jack Roubos and M. H. Groenendijk, the project planners. They contributed to the current planning practice described in the thesis. Thanks to Wim Smit, the group leader of work preparation, and Maarten Gravestein, the outfit coordinator, who shared their knowledge about small iron work and equipment installation. I am also grateful to Johan de Bruin, the manager of block assembly. Without his helpful assistance, I hardly could have obtained such detailed contact information of co-makers of IHC Offshore \& Marine B.V.. 
206 Automatic generation of assembly sequence for the planning of outfitting processes in shipbuilding

During the research stage, I met people from different subcontracted companies of IHC Offshore \& Marine B.V., who generously shared their experience and knowledge of outfitting work. They are: Wim van Ham, the foreman of IHC Piping B.V., and his team members, Arie v.d. Velden and Yuan C. Mosteiro; Bram de Jong, the construction supervisor of Heinen \& Hopman Engineering B.V.; Danny de Bruin and Andries van Eijl, the electrical supervisors of Combination Croon Bakker v.o.f.; Bart van der Aa, the manager of Bawavi Constructiewerkplaats B.V.; Kees Kok, the managing director of Minks Kunststoftechniek B.V; Vladimir Kavedzija, the project coordinator of Johnson Controls Systems \& Service B.V.. Many thanks to all of them.

Heartfelt thanks to people from different organizations who helped me do the final model validation. Jenny Coenen, dear colleague from TU Delft, and Kees Meijer, ex-colleague and now working at IHC Offshore \& Marine B.V., not only helped to organize several validation meetings but also were present in these meetings to make sure that the conversations mixing English and Dutch were understandable. During the validation stage, Joop Lemson, the deputy site manager production of IHC Dredgers B.V., Atila Batman, the production project manager of IHC Dredgers B.V., Rob van Vugt, the production supervisor of IHC Dredgers B.V., Arjan van Eijl, the outfitting coordinator of IHC Offshore \& Marine B.V. and Frank Verhelst, the project manager of Damen Schelde Naval Shipbuilding, provided invaluable feedbacks and practical suggestions. Many thanks for their contributions.

I would like to thank all members of the $\mathrm{PhD}$ commission for being willing to be in my committee and for their helpful comments on the draft version of this thesis.

I am deeply indebted to all my church friends in the Netherlands, for their constant support. Without their love, prayers and tender cares, I could not go so far. No words can adequately express my gratitude to my parents, for their continuously unconditional love, care and support during my years in the Netherlands. Last but not least, my deepest gratitude goes to my Lord Jesus, for His precious presence, which greatly comforts me through my life stages. Honor and glory to Him! 


\section{Curriculum Vitae}

Yan Wei was born on the 21st of March, 1982, in Xiangfan, Hubei province, P. R. China. She went to Wuhan University of Technology in Wuhan in September, 2000 and obtained a bachelor degree in 'Ships and Marine Engineering' in June, 2004. Afterwards, she continued her master study in the same university. In June, 2007, she obtained a Master of Engineering degree in 'Design and Manufacture of Ships and Marine Structures'. In October 2007 she started her PhD research in Delft University of Technology, of which the results are presented in this dissertation. 